\title{
Pathways for phospholipid deacylation in Saccharomyces cerevisiae and their impact on fatty acid trafficking and equilibrium
}

\author{
Ph.D. Thesis \\ in partial fulfillment of the requirements \\ for the degree "Doctor of Philosophy (Ph.D.)" \\ in the Molecular Biology Program \\ at the Georg August University Göttingen, \\ Faculty of Biology
}

\author{
submitted by \\ Gabriel Eduardo Mora Oberländer \\ born in \\ Bogotá, Colombia \\ Department for Plant Biochemistry \\ Albrecht-von-Haller-Institute for Plant Sciences \\ Georg August University Göttingen
}



First Referee: $\quad$ Prof. Dr. Ivo Feußner

Second Referee: Prof. Dr. Michael Thumm 



\section{Declaration}

I hereby declare that the PhD thesis entitled "Pathways for phospholipid deacylation in Saccharomyces cerevisiae and their impact on fatty acid trafficking and equilibrium" has been written independently and with no other sources and aids than quoted.

Gabriel Mora

Göttingen, January 29, 2010 



\section{Table of Contents}

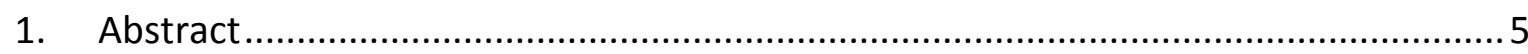

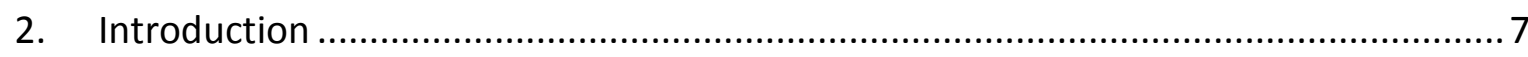

2.1. Membrane function and phospholipid composition ...................................... 7

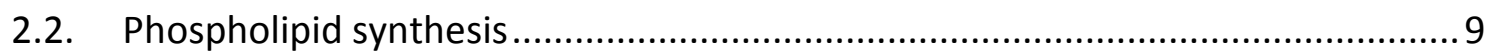

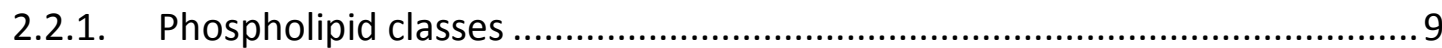

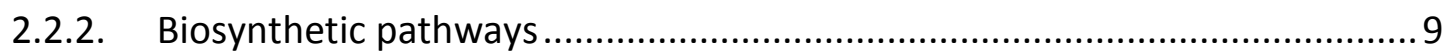

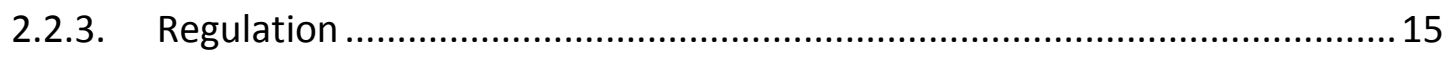

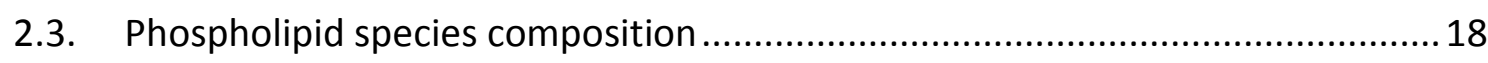

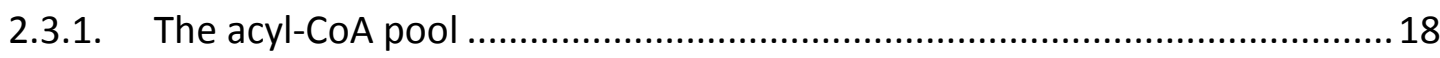

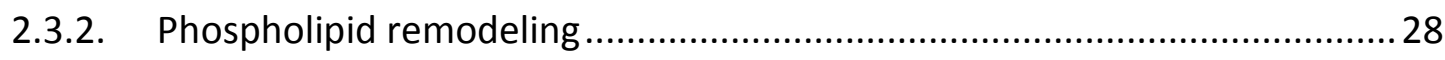

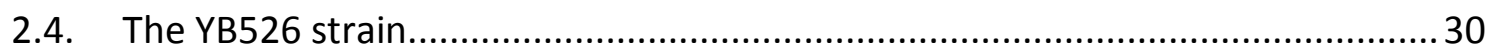

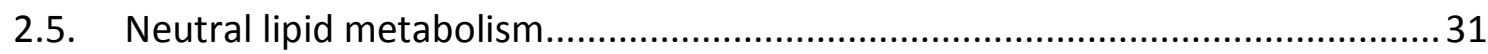

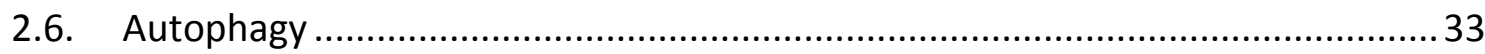

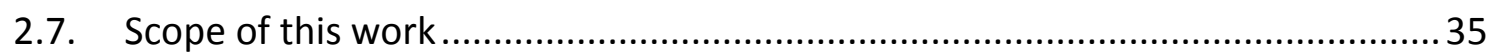

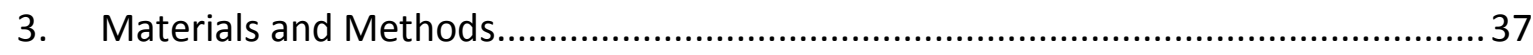

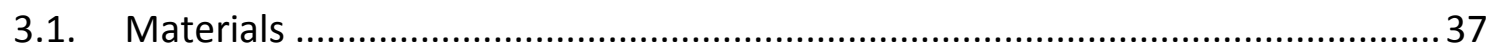

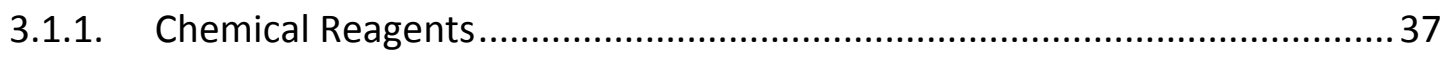

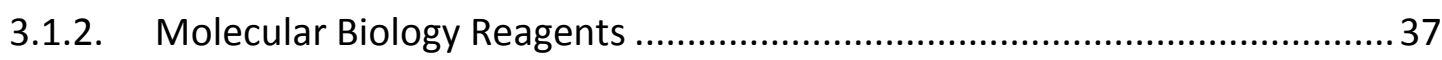

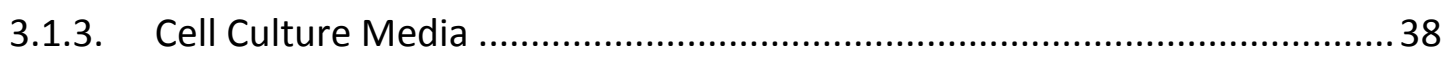

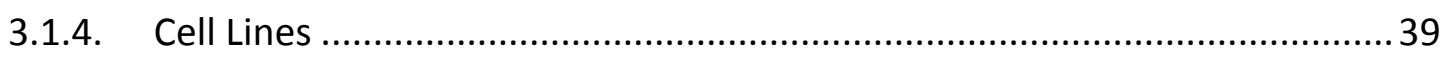

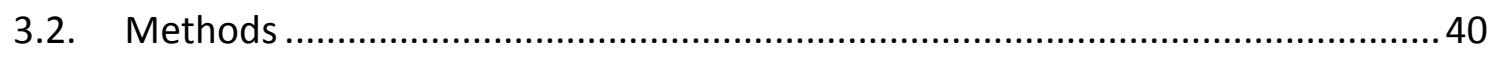

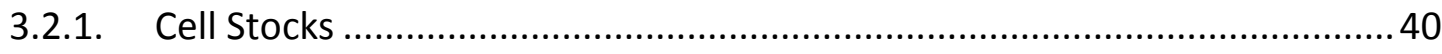

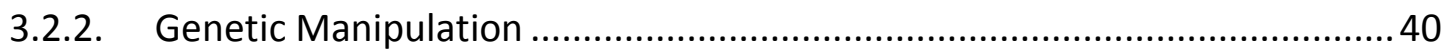


3.2.3. Free and Bound Fatty Acid Profile Analysis ........................................... 51

3.2.4. Analysis of FA in Specific Lipid Classes..................................................5

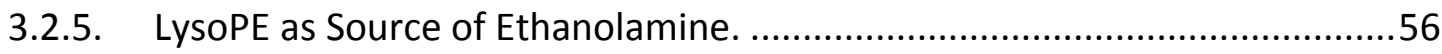

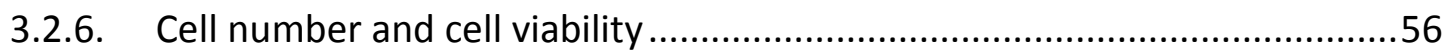

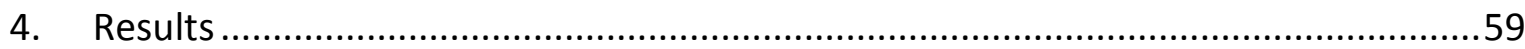

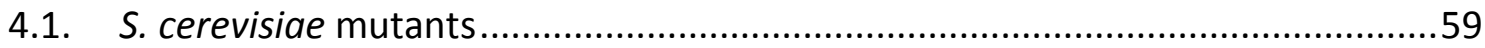

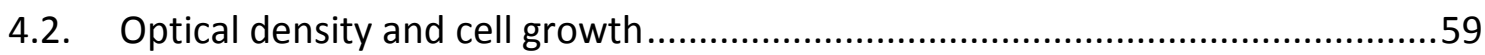

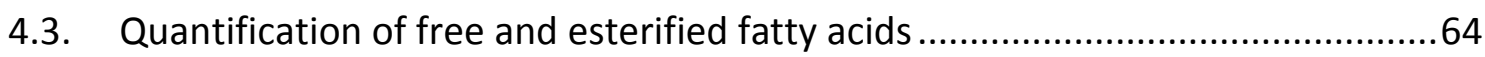

4.3.1. Multiple candidate pathways for PL deacylation: PLBs, NL metabolism, PLD, LPLATs, autophagy and the peroxisome...............................................................64

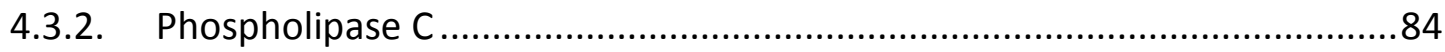

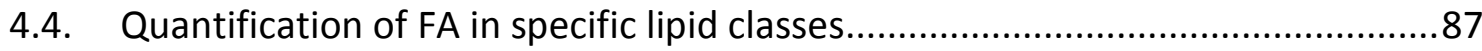

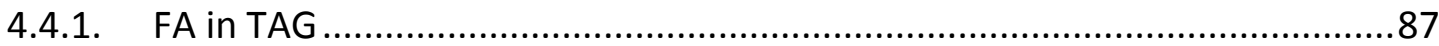

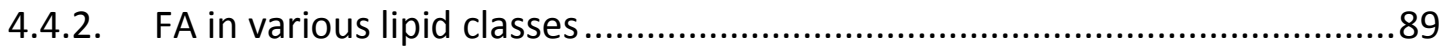

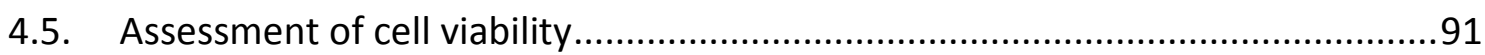

4.6. LysoPE as a source of ethanolamine in PLB deficient cells .............................92

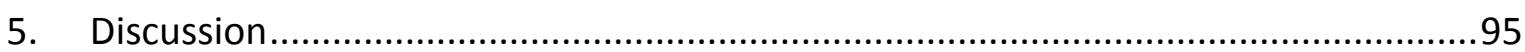

5.1. Potential mechanisms for lipid deacylation and their impact on the equilibrium

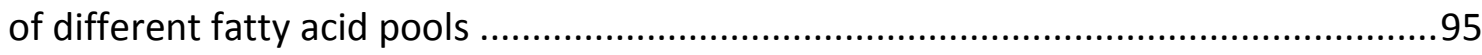

5.1.1. Free and bound fatty acid pools in acyl-CoA synthetase deficient cells.......95

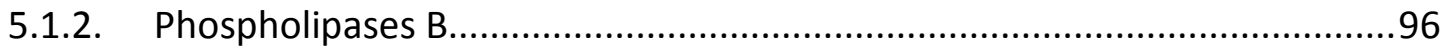

5.1.3. Neutral lipid metabolism in PL synthesis and remodeling .........................98

5.1.4. Phospholipases C, D, and lysophospholipid acyltransferases ...................106

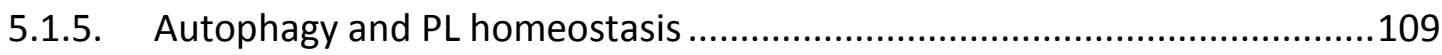

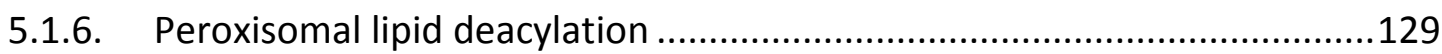

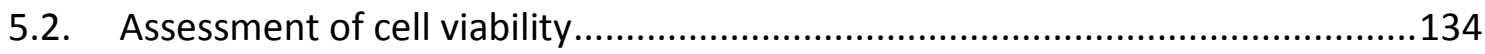




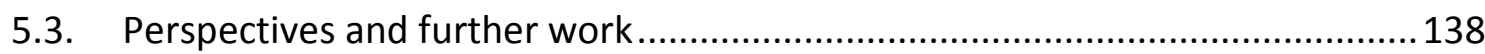

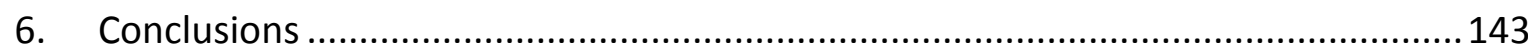

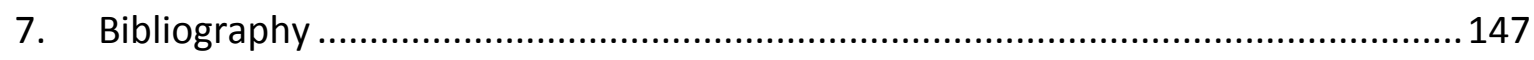

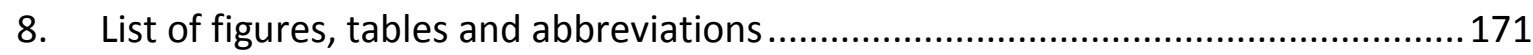

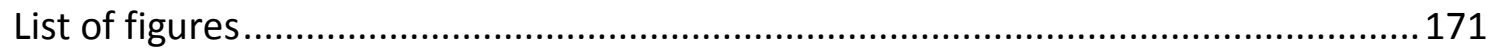

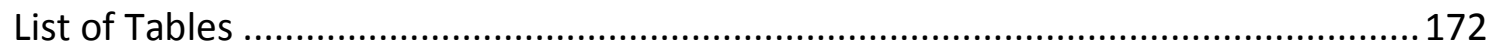

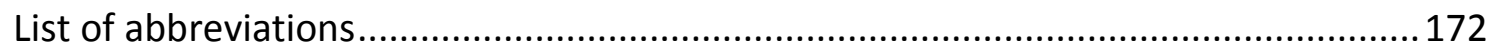

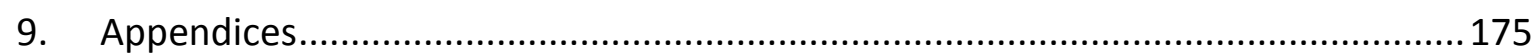

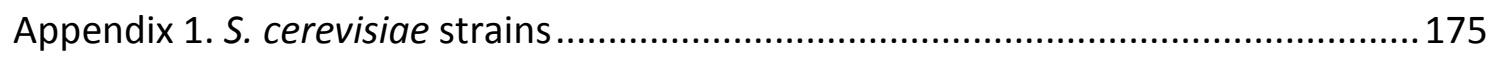

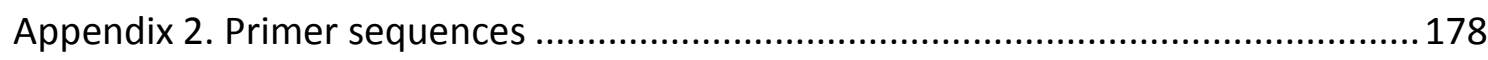

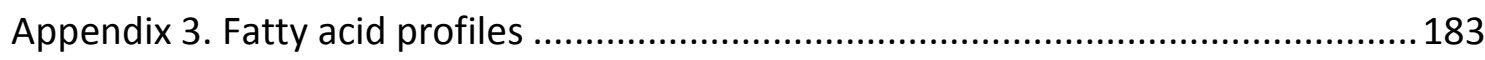

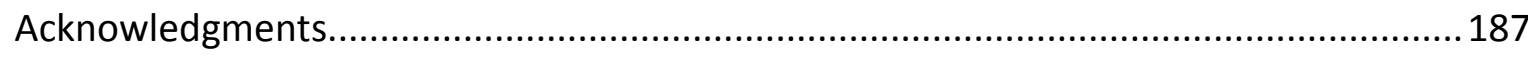





\section{Abstract}

The phospholipid (PL) class and species composition is a fundamental characteristic of membranes, essential to their proper function. The PL species distribution of cellular lipids is only partly achieved through de novo synthesis; PL degradation and PL remodeling are known to occur extensively and to be fundamental in the establishment of the steady state lipid composition. In the yeast Saccharomyces cerevisiae the pathways for PL deacylation, leading to PL degradation or PL remodeling, remained, until now, unidentified. The difficulty in the identification of the enzymatic activities involved lies, partly, in the fact that free fatty acids (FFA), lysophospholipids and glycerophosphodiesters (GPD), the direct products of $\mathrm{PL}$ deacylation, are rapidly metabolized and are therefore inaccessible to accurate experimental detection and quantification.

In the case of FFA, metabolization is necessarily initiated by activation through conversion to acyl-CoA, a reaction catalyzed by acyl-CoA synthetases (ACS). The $S$. cerevisiae strain YB526, in which the genes coding for the ACS enzymes Faa1p, Faa2p, Faa3p and Faa4p have been deleted, is unable to activate, and therefore to metabolize, FFA. In consequence, FFA derived from lipid deacylation accumulate in this strain and can be quantified. An analysis of the effect that the interference with specific enzymatic activities and metabolic processes has on the FFA content of YB526 cells, reveals the role of those activities and processes in lipid deacylation and in the homeostasis of fatty acids (FA) and acyl-ester pools in general.

This approach has revealed that phospholipases B are not involved in PL remodeling or other constitutive forms of PL deacylation. Autophagic degradation, in contrast, was identified as the quantitatively most prominent mechanism for PL deacylation. Despite the fact that autophagy constitutes a mechanism for bulk degradation, its role in the regulation of the cellular PL content appears to be not only quantitative, but also qualitative. Neutral lipid (NL) metabolism was identified as well as a central element in the regulation of PL species composition. NL metabolism as a whole, mediates FA 
trafficking to a quantitatively similar extent than autophagy. Within NL metabolism, the phospholipid:DAG acyltransferase (PDAT) Lro1p makes a substantial contribution to PL deacylation, and is suggested by our results as the most likely mediator in PL remodeling. The acyl-CoA dependant biosynthetic activities of NL metabolism appear to be involved as well in the establishment of PL species composition through a modulation of the substrate available for PL synthesis. Furthermore, we established that impaired NL synthesis leads to augmented autophagic degradation of $\mathrm{PL}$, while impaired sequestration of substrate for autophagic degradation leads to enhanced NL synthesis. 


\section{Introduction}

\subsection{Membrane function and phospholipid composition}

One of the most prominent cellular functions of lipids is the formation of membranes, providing a barrier against free diffusion and allowing for the establishment of the distinct chemical environment that constitutes the cell and the organellar subenvironments which define eukaryotic cells. Membranes, however, are much more than a separation between spaces. Every membrane is an environment on its own, hosting a variety of proteins that control the translocation of matter across the barrier and catalyze or regulate metabolic reactions and signal transduction events. The lipid composition of membranes must therefore match not only structural properties to guarantee membrane integrity while still allowing for a dynamic behavior, but must also provide the particular physicochemical conditions required for the specific presence and function of defined sets of proteins, giving rise to membrane identity; the regulation of membrane lipid composition is, in consequence, a critical cellular task.

In the present work we apply a novel approach (a description of which is provided in section 2.4.) to evaluate the role of several enzymes, and the cellular processes they make part of, in the metabolic trafficking of fatty acids (FA), a central element in the regulation of membrane lipid composition. Our study is conducted with emphasis on, but not limited to, the identification of enzymatic activities involved in phospholipid (PL) deacylation. We utilize the eukaryotic model organism S. cerevisiae. Differences between lipid metabolism in the yeast S. cerevisiae and in higher eukaryotes do exist, the most prominent and relevant of which will be mentioned within the text; however, the similarities in terms of gene homology, architecture of the major metabolic pathways, and regulatory mechanisms prevail over the divergences (Gaspar et al. 2007). Within this document, the term "yeast", which rigorously refers to unicellular fungi in general, is used in specific reference to S. cerevisiae.

Altogether the diversity of lipid molecular species in eukaryotic cells is estimated to be above one thousand (van Meer 2005; Gaspar et al. 2007; Dowhan 1997). The complexity 
in the regulation of this enormous molecular array is further increased by the fact that lipids, as well as their precursors and degradation products, play several roles in mechanisms beyond membrane formation, such as signaling, traffic targeting, cell cycle development, protein modification and energy storage (van Meer 2005; Gaspar et al. 2007; Dowhan et al. 2004). Sterols, sphingolipids and glycolipids ${ }^{1}$ are essential to the structure and function of some membranes, phospholipids, in contrast, are essential to all known cellular membranes.

The pathways for de novo PL synthesis leading to the formation of different PL classes are well understood, most of the enzymes involved have been identified and abundant information about their regulation is available (section 2.2.). PL homeostasis, however, does not only refer to class distribution. The species profile, i.e. their acyl chain composition, plays a prominent role in fundamental membrane properties such as fluidity and intrinsic curvature. As will be described with more detail in section 2.3., the regulation of de novo PL synthesis and the substrate selectivity of the enzymes involved cannot, on their own, account for the cellular steady state PL species profile. Establishing, maintaining and, when necessary, modifying this profile requires, on the one hand, regulation in the species composition of the substrate available to feed into the biosynthetic pathway, i.e. the acyl-Coenzyme A (acyl-CoA) pool, and on the other hand, extensive degradation and/or remodeling (acyl editing) of de novo synthesized PL. In contrast to PL synthesis, the mechanisms for PL deacylation in S. cerevisiae are still largely unknown, as is then, of course, their impact on FA availability. The homeostasis of FA is, in fact, a problem as complex and as central as that of PL. The pool of free fatty acids (FFA) is tightly connected to the acyl-CoA pool, which comprises the metabolically relevant form of fatty acids. The pathways either feeding into the acyl-CoA pool, such as de novo FA synthesis, FA activation, neutral lipid (NL) mobilization and FA import, or depleting it, like lipid synthesis and $\beta$-oxidation are understood to different extents. The way, however, in which these processes are balanced for the establishment and maintenance of the required acyl-CoA pool, is scarcely understood.

\footnotetext{
1 Glycolipids in S. cerevisiae are mainly sphingolipids and glycosylated glycerophospholipids (glycosylphosphatidylinositol). Glycoglycerolipids, common and essential in many organisms, are almost absent from yeast. Minor amounts of rare glycolipids (Steryl glycosides, Acyl glucoses) have been reported (Rattray et al. 1975; Työrinoja et al. 1974).
} 


\subsection{Phospholipid synthesis}

\subsubsection{Phospholipid classes}

The major phospholipids present in $S$. cerevisiae are phosphatidylcholine (PC), phosphatidylethanolamine (PE), phosphatidylinositol (PI) and phosphatidylserine (PS); phosphatidylglycerol (PG) and cardiolipin (CL) are also present but restricted to mitochondrial membranes (Paltauf et al. 1992; Carman and Zeimetz 1996; Carman and Henry 1989; Carman and Henry 1999; Rattray et al. 1975). Yeast cells are actually very tolerant to dramatic variations in their PL composition, variations that can easily be induced through culture or genetic conditions (Carman and Zeimetz 1996). PC, for example, is generally the most abundant $\mathrm{PL}$, representing up to $50 \%$ of the total (Kent and Carman 1999); however, cells deficient in the biosynthesis of choline do not loose viability in absence of an external choline source until the cellular PC level drops beneath 2\% (Boumann et al. 2006). In fact, PC can be absent altogether, if at least minor amounts of its precursors (mono- or di-methylated PE) are present (Boumann et al. 2006; McGraw and Henry 1989). PS, PG and CL are also dispensable, although their absence leads to mitochondrial and respiratory malfunctions (Atkinson et al. 1980a; Atkinson et al. 1980b; Chang et al. 1998a). PE and PI, in contrast, are essential (Nikawa et al. 1987; Storey et al. 2001; Birner et al. 2001). Changes induced in the content of a particular PL through media or genetic manipulations are always accompanied by changes in the content and species distribution of other phospholipids. These changes aim to compensate two major characteristics: membrane charge (Becker and Lester 1977), involved in proteinmembrane interactions (McLaughlin and Murray 2005; Yeung et al. 2008), and the proportion of bilayer to non-bilayer forming lipids (Rietveld et al. 1993; Boumann et al. 2006; Koynova and Caffrey 1994; Morein et al. 1996), a parameter which is essential to membrane stability, fission, fusion and function of integral membrane proteins (van Den Brink-van der Laan et al. 2004; Yeagle 1989).

\subsubsection{Biosynthetic pathways}

The pivotal point in the synthesis of all phospholipids, as well as in the synthesis of the non-phosphorylated glycerolipids diacylglycerol (DAG) and triacylglycerol (TAG), is 
phosphatidic acid (PA) (figure 1). In yeast, the initial step of de novo PA synthesis utilizes acyl-CoA and either glycerol-3-phosphate (G3P) or dihydroxyacetone phosphate (DHAP), respectively producing 1-acylglycerol-3-phosphate (lysophosphatidic acid; LPA) and 1acyl-DHAP (Schlossman and Bell 1978; Athenstaedt et al. 1999a). 1-acyl-DHAP is then converted to LPA, at the expense of NADPH oxidation, by the acyl-DHAP reductase Ayr1p localized in the endoplasmic reticulum (ER) and lipid particles (LP) (Athenstaedt and Daum 2000; Athenstaedt and Daum 1999; Racenis et al. 1992). This initial acylation is catalyzed by the G3P/DHAP dual substrate acyltransferases Gat1p and Gat2p (Zheng and Zou 2001). Although either of them is sufficient to sustain cell viability without obvious growth defects, they make different contributions to lipid synthesis. Gat1p, localized both in the ER and in LP, efficiently utilizes 16- and 18-carbon FA, with some preference for unsaturated ones, and does not discriminate between G3P and DHAP; Gat2p, present only in the ER, acylates G3P with higher efficiency than DHAP and has a marked preference for 16-carbon, mostly unsaturated, FA (Zheng and Zou 2001). Cells lacking Gat1p present an increase of $50 \%$ in TAG synthesis and a 5 -fold increase in the deacylation of PC derived from the CDP-choline pathway, while cells without Gat2p present a $50 \%$ decrease in TAG synthesis and a 10 -fold drop in the deacylation of CDPcholine derived PC (Zaremberg and McMaster 2002). The presence of an additional, so far unidentified, transferase in the mitochondria which prefers DAHP as a substrate has been suggested (Athenstaedt et al. 1999a), but its actual existence has been (inconclusively) disputed (Zaremberg and McMaster 2002). If this activity is in fact present its contribution to lipid synthesis is insufficient to sustain cell viability, since GAT1 and GAT2 deletions are synthetically lethal (Zheng and Zou 2001) and it is expected to make only a minimal contribution to lipid synthesis.

LPA does not accumulate to any large extent in the cells; it is instead rapidly converted to PA through transfer of a second acyl chain to its $s n-2$ position by a LPA acyl transferase (LPAAT) (figure 1). Slc1p (Nagiec et al. 1993) and Ale1p (Tamaki et al. 2007; Benghezal et al. 2007; Chen et al. 2007a; Jain et al. 2007; Riekhof et al. 2007) are the major LPAAT enzymes of yeast; however their relative contribution to PA and PL synthesis in vivo is not entirely clear. Some studies (Tamaki et al. 2007; Athenstaedt and Daum 1997; Chen et al. 2007a) have reported a reduction in LPAAT activity upon SLC1 inactivation of 30 to $50 \%$, 
while others report no change (Riekhof et al. 2007). Upon ALE1 deletion Riekhof and coworkers report an $85 \%$ decrease in LPAAT activity, while the group of Chen reports no change (Chen et al. 2007a). For the more general lysophospholipid acyl transferase activity (LPLAT), Jain and coworkers (Jain et al. 2007) found a 60\% decrease in absence of SLC1 but no significant change when ALE1 was deleted, while the group of Tamaki reports only a minor decrease of LPLAT activity in absence of SLC1 and a substantial one in absence of ALE1 (Tamaki et al. 2007). These notorious confusion and contradictions might arise from at least three facts. First, the distinction between LPAAT and LPLAT activities is not always made clear, even if one or the other term is used. LPLAT activity refers to a broader and more general set of reactions within which LPAAT and the acylation of other lysoglycerolipids are included. However, if assays rely on the exogenous provision of lysolipid substrates LPLAT determinations become excluding. Second, these activities are determined through one of two alternative methods: Release of coenzyme A (CoA) or incorporation of labeled fatty acids. In the former case other acyl-CoA dependant transferase activities might interfere, despite efforts to generate a CoA release baseline for the assay; in the later, the result will be strongly dependant on the provided substrate and the substrate preferences of the enzymes. Third, the relative contribution of the two enzymes could vary with the stage and conditions of the culture. Regardless of this, either of this two LPAAT enzymes is sufficient to sustain cellular growth alone and, in fact, only minor variations in overall cellular lipid profiles result from deleting one of them (Benghezal et al. 2007). Slc1p has a preference for long (mainly 18- but also 22- and 24-carbon) unsaturated fatty acids but it does not entirely exclude shorter or saturated ones (Athenstaedt and Daum 1999; Jain et al. 2007). Ale1p is strongly selective for unsaturated FA, but does not appear to discriminate for chain length (Tamaki et al. 2007; Jain et al. 2007). As mentioned above, these two enzymes can acylate other lysolipids beyond LPA. SIc1p acylates lysoPI and lysoPS (Benghezal et al. 2007), while Ale1p accepts lysoPC, lysoPE, lysoPI, lysoPS and lysoPG (Tamaki et al. 2007; Benghezal et al. 2007; Chen et al. 2007a; Jain et al. 2007; Riekhof et al. 2007). The substrate preference of the enzymes with respect to the acyl chain in the sn-1 position of the lysolipids has not yet been extensively studied. Data is available for Ale1p and lysoPC where a dramatic, and surprising, preference for an unsaturated chain at the $s n-1$ position was found (Tamaki et al. 2007). In addition to SIc1p and Ale1p, Ict1p was also 
recently identified as an acyl-CoA dependant LPAAT enzyme. Ict1p does not acylate other lysolipids beyond LPA and preferentially utilizes 18:1 fatty acids (Ghosh et al. 2008a). The deletions of SLC1 and ALE1 are synthetic lethal, indicating that Ict1p is insufficient to fulfill the PA synthesis requirement of the cell. The major role of Ict1p appears to be the synthesis of lipids to cope with membrane damage caused by presence of organic solvents in the media, however, deletion of ICT1 leads to a reduced content of PA and other PL even in absence of such stress (Ghosh et al. 2008a). In contrast to Slc1p, which is localized in the ER and LP (Athenstaedt and Daum 1997), and Ale1p, which resides mainly in the mitochondrial associated membrane of the ER (MAM) (Tamaki et al. 2007; Riekhof et al. 2007; Huh et al. 2003), Ict1p is a soluble cytosolic enzyme (Ghosh et al. 2008a; Ghosh et al. 2008b).

Following from PA, PL synthesis can proceed through two alternative pathways: the cytidine diphosphate diacylglycerol (CDP-DAG) pathway (Steiner and Lester 1970; Steiner and Lester 1972a; Steiner and Lester 1972b) and the DAG pathway, also known as Kennedy pathway (Kennedy and Weiss 1956) (figure 1).

In yeast, under standard conditions, the majority of the cells' PL are synthesized through the CDP-DAG pathway (Paltauf et al. 1992; Carman and Henry 1989; Daum et al. 1998; Henry and Patton-Vogt 1998). In this pathway PA is converted to CDP-DAG in a CTP dependant reaction catalyzed by Cds1p (Shen et al. 1996; Dowhan 1997), an enzyme localized in the ER and mitochondria (Zinser et al. 1991; Kuchler et al. 1986; Kelley and Carman 1987). CDP-DAG is fed into three competitive branches of the pathway. In the first one, PI is produced from CDP-DAG and inositol by the PI synthase Pis1p. This protein was originally believed to be localized on the outer mitochondrial membrane and on microsomes (Fischl and Carman 1983; Nikawa and Yamashita 1997; Nikawa et al. 1987; Kuchler et al. 1986), a more recent large scale protein localization endeavor found Pis1p on the ER, Golgi and in vesicles (Natter et al. 2005). Currently, the main site of Pis1p action is believed to be the ER, where it is present as an associated protein (Habeler et al. 2002; Han et al. 2005). Some of the PI produced is then utilized in the synthesis of glycosylphosphatidylinositol (GPI) anchors (Conzelmann et al. 1990; Eisenhaber et al. 2003), inositol-containing sphingolipids (Becker and Lester 1980; Lester and Dickson 1993) and polyphosphoinositides (Flanagan et al. 1993; Garcia-Bustos et al. 1994). In the 
second branch, PS is produced from CDP-DAG and serine by the MAM localized phosphatidylserine synthase Pss1p, ${ }^{2}$ better known as Cho1p (Gaigg et al. 1995; Bae-Lee and Carman 1984; Yamashita and Nikawa 1997; Letts et al. 1983). In the later stages of this branch the serine head group in PS is decarboxylated to yield PE and, finally, the ethanolamine head group in PE is methylated yielding PC. PS decarboxylation is mainly mediated by the PS decarboxylase Psd1p, localized in mitochondria (Clancey et al. 1993; Kuchler et al. 1986), and secondarily by Psd2p, localized in the Golgi and vacuole (Voelker 1997; Trotter and Voelker 1995). PE is converted to PC by the ER localized PE methyltransferases Pem1p and Pem2p (also known as Cho2p and Opi3p, respectively) (Kanipes and Henry 1997; Kodaki and Yamashita 1987). Cho2p is essentially responsible for the first methylation step, while Opi3p normally introduces the second and third methyl groups; however, in absence of Cho2p, Opi3p can produce small amounts of monomethyl-PE (MMPE) which it then turns into dimethyl-PE (DMPE) and subsequently into PC. This small amount of PC is sufficient to sustain cellular growth and cho2 $\Delta$ cells are therefore not choline auxotrophs. In opi3 $\Delta$ strains no PC is formed through this pathway but the MMPE that accumulates can substitute for it and the cells are again no choline auxotrophs (Summers et al. 1988; McGraw and Henry 1989; Kodaki and Yamashita 1987; Kodaki and Yamashita 1989). In the third branch of the CDP-DAG pathway, the phosphatidylglycerol phosphate (PGP) synthase encoded by the gene PGS1 adds G3P to CDP-DAG, forming PGP (Janitor and Subík 1993; Chang et al. 1998). PGP is then dephosphorylated to PG by a so far unidentified phosphatase. Finally, a second molecule of CDP-DAG is added to PG by the cardiolipin synthase Crd1p, producing $\mathrm{CL}$ (Jiang et al. 1997; Tuller et al. 1998; Chang et al. 1998b).Pgs1p and Crd1p activities are mainly localized in the inner mitochondrial membrane, although minor Pgs1p activity has been found on microsomes (Zinser et al. 1991; Kuchler et al. 1986).

The second pathway for PL synthesis, known as Kennedy pathway, has two branches: the CDP-ethanolamine and CDP-choline branches. Initially, ethanolamine and choline are phosphorylated by the ethanolamine and choline kinases Eki1p and Cki1p respectively (although Eki1p can also contribute to choline phosphorylation and Cki1p to

\footnotetext{
${ }^{2}$ In contrast to the situation in yeast, PS synthesis in mammals proceeds through an exchange of serine with PC or PE (Kuge and Nishijima 1997).
} 
ethanolamine phosphorylation) (Kim et al. 1998; Hosaka et al. 1989; Kim et al. 1999a; Yamashita and Nikawa 1997). Ethanolamine- and cholinephosphate are then converted to CDP-ethanolamine and CDP-choline by the cytidylytransferases Ect1p and Pct1p respectively (Nakashima et al. 1997; Min-Seok et al. 1996; Tsukagoshi et al. 1987). Finally, PE and PC are synthesized at the ER by the CDP-ethanolamine:1,2-diacylglycerol ethanolaminephosphotransferase Ept1p and the CDP-choline:1,2-diacylglycerol cholinephosphotransferase Cpt1p (McMaster and Bell 1997; Hjelmstad and Bell 1987; Hjelmstad and Bell 1988).

In absence of exogenous choline or ethanolamine the Kennedy pathway is still active, as PC synthesized through the CDP-DAG pathway is permanently hydrolyzed by phospholipase D activity (Spo14p) and the choline produced is recycled by the CDPcholine branch (Sreenivas et al. 1998; Carman and Henry 1989; Patton-Vogt et al. 1997; Henry and Patton-Vogt 1998); however, the pathway is not essential for cell survival. In contrast, complete disruption of the CDP-DAG pathway by deletion of CDS1 or of the PI branch of this pathway by deletion of PIS1 is lethal as no alternative route for PI synthesis is available (Daum et al. 1998; Nikawa et al. 1987). Disruption of the PS branch is not lethal since PS is not essential, but turns the cells into ethanolamine auxotrophs (or choline auxotrophs if the disruption takes place in the final PE methylation steps) and makes their survival dependant on a functional Kennedy pathway (Paltauf et al. 1992; Carman and Zeimetz 1996; Carman and Henry 1989). 


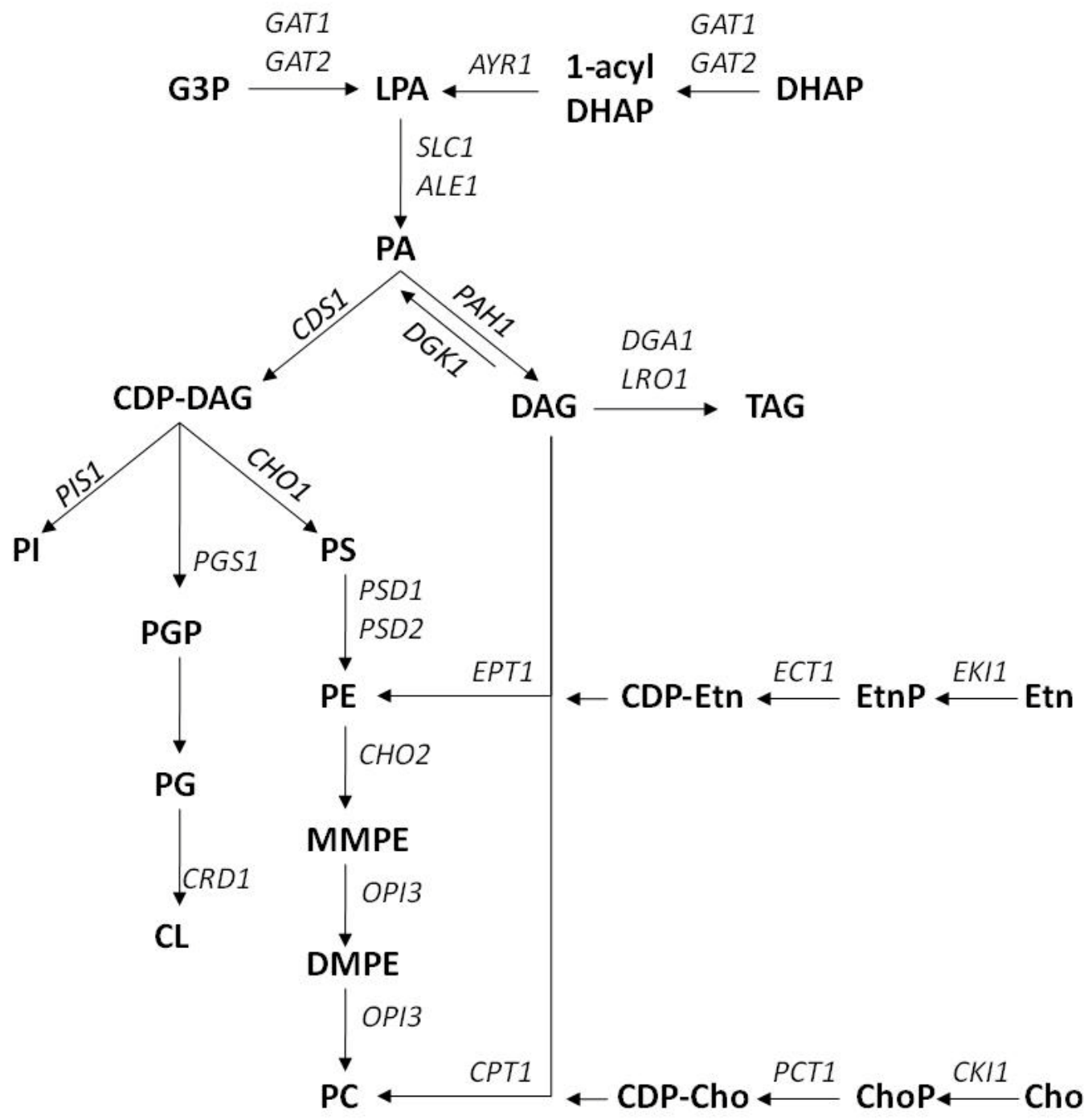

Figure 1. Synthesis of glycerolipids. Details are described in the main text. The acylations catalyzed by the acyl transferases Gat1p, Gat2p, Slc1p, Ale1p and Dga1p depend on acyl-CoA; Lro1p utilizes the acyl chain of a PL. Reduction by Ayr1p requires NADPH. Activation of PA, EtnP, and ChoP by Cds1p, Ect1p and Pct1p, respectively, depends on CTP. Phosphorylation of DAG by Dgk1p also depends on CTP. Etn and Cho phosphorylation by Eki1p and Cki1p consume ATP. In addition to the backbone, shown in the figure, the synthases Pis1p, Cho1p, Pgs1p and Crd1p, respectively require inositol, serine, G3P and CDP-DAG. Cho2p and Opi3p utilize S-adenosyl methionine as methyl donor. The phosphatase mediating PGP to PG conversion has not been identified.

\subsubsection{Regulation}

The regulation of PL synthesis is, as synthesis itself, centered on PA. Many of the genes involved in PL synthesis (CDS1, CHO1, PSD1, CHO2, OPI3, EKI1, EPT1, CKI1, CPT1, INO1) have a UAS INO element in their promoter region (Carman and Han 2009a). The 
Ino2p/Ino4p complex binds to this element and activates their transcription, leading to enhanced PL synthesis (Nikoloff and Henry 1994; Ambroziak and Henry 1994; Lopes and Henry 1991; Bachhawat et al. 1995; Schwank et al. 1995). When the regulatory protein Opi1p is present inside the nucleus, the interaction of the Ino2p/Ino4p complex with the $\mathrm{UAS}_{\mathrm{INO}}$ promoter element is disrupted, gene transcription is repressed and PL synthesis is down regulated. Direct binding of Ino $2 p$ by Opi1p is considered to be the most likely mechanism mediating repression (Carman and Han 2009a; Chen et al. 2007b); however, while strong evidence of this direct interaction has been obtained in vitro (Wagner et al. 2001; Gardenour et al. 2004; Dietz et al. 2003; Kaadige and Lopes 2006; Heyken et al. 2005), evidence against it in vivo has also been presented (Graves and Henry 2000) and the matter cannot be considered as entirely resolved. Opi1p binds PA and the ER/nuclear membrane localized protein Scs2p (Gavin et al. 2002; Loewen et al. 2004; Loewen et al. 2003). Therefore, a high PA level retains Opi3p outside the nucleus preventing it from down regulating the expression of $\mathrm{PL}$ synthesis genes and leading to increased $\mathrm{PL}$ synthesis, while a low PA level releases Opi3p and leads to repression of PL synthesis (Loewen et al. 2004; Loewen and Levine 2005; Carman and Henry 2007; Chen et al. 2007b).

The PA content is regulated by several media conditions, among which the availability of inositol and zinc are the best understood. If not provided in the media, inositol is produced from glucose-6-phosphate by the inositol-3-phosphate synthase Ino1p, the expression of which is itself under $\mathrm{UAS}_{\mathrm{INO}}$ control. Inositol supplementation inhibits the activities of Cho1p and Pgs1p leading to reduced PS and CL synthesis, but enhances PI synthesis (Kelley et al. 1988; Greenberg et al. 1988). The high rate of PI synthesis depletes the PA pool releasing Opi1p from the ER and leading to the repression of INO1,

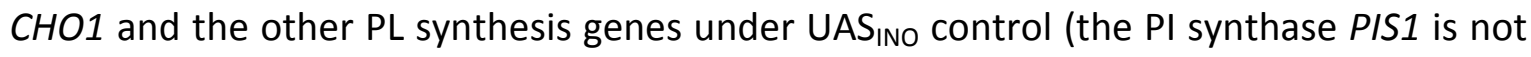
under UAS INO control) and ultimately results in cells with a high PI content and a decreased PS, CL and PC content (Loewen et al. 2004; Carman and Henry 1999; Carman and Henry 2007; Kelley et al. 1988). Note that while inositol inhibits both, the activity and the transcription of Cho1p, the inositol derived repression of CL synthesis is mediated by Pgs1p phosphorylation but not by a repression of its transcription (He and Greenberg 2004; Zhong and Greenberg 2003). 
Instead of a UAS INO element, the PIS1 promoter contains a UAS ZRE element. Under low zinc conditions the expression of the transcriptional regulator Zap1p is induced. Zap1p binds to $\mathrm{UAS}_{\mathrm{ZRE}}$ elements and up regulates the expression of the genes under its control, among which, in addition to PIS1, are ZAP1 itself and the zinc transporters ZRT1, ZRT2, ZRT3 and FET4 (Waters and Eide 2002; MacDiarmid et al. 2000; Eide 2003; Zhao and Eide 1996a; Zhao and Eide 1996b; Zhao and Eide 1997; Zhao et al. 1998; Han et al. 2005). The high expression of Pis1p induced by Zap1p leads to elevated PI synthesis, which in turn results in the depletion of the PA pool and the repression of PS synthesis by release of Opi1p (Carman and Han 2007; Han et al. 2005; Iwanyshyn et al. 2004). Synthesis of PC and PE through the CDP-DAG pathway is also repressed under these circumstances; however, the CKI1 and EKI1 promoters have a UAS element, Zap1p induction overrules Op1p repression and results in a higher rate of PC and PE synthesis through the Kennedy pathway (Soto and Carman 2008; Kersting and Carman 2006). In the case of PC, but not in that of PE, this increase compensates the loss of synthesis through the CDP-DAG pathway and ultimately results in cells with a high content of PI, an unaffected PC content, and low levels of PS and PE (Iwanyshyn et al. 2004).

In addition to the rate of PI synthesis, the phosphorylation and dephosphorylation equilibrium between DAG and PA is also central to the regulation of PA levels and, furthermore, to the channeling of PA into either the CDP-DAG pathway, the Kennedy pathway, or TAG synthesis. The PA phosphatase Pah1p turns PA into DAG (Han et al. 2006), while the DAG kinase Dgk1p turns DAG into PA (Han et al. 2008a; Han et al. 2008b). High levels of CDP-DAG, CL or PI enhance Pah1p activity (Wu and Carman 1996) while CDP-DAG inhibits Dgk1p (Han et al. 2008a) shifting the equilibrium towards DAG and therefore towards PE and PC synthesis through the Kennedy pathway. High levels of Pah1p activity eventually lead to low PA content, a repression of both pathways for PL synthesis and preferential TAG synthesis (Carman and Han 2009b; Wu and Carman 1996). In contrast to CDP-DAG, its precursor CTP (which is also the phosphate donor for DAG phosphorylation and the precursor of CDP-choline and CDP-ethanolamine (Han et al. 2008a; Chang and Carman 2008)) inhibits Pah1p and stimulates Dgk1p, leading to higher PA levels and derepression of PL synthesis (Chang and Carman 2008; Han et al. 2008a; 
Ostrander et al. 1998; $\mathrm{Wu}$ and Carman 1994). Pah1p is a $\mathrm{Mg}^{2+}$-dependent cytosolic enzyme (although it associates with membranes peripherally) (Huh et al. 2003) and, as described above, is central in de novo PL synthesis. In addition to Pah1p, two $\mathrm{Mg}^{2+}$ independent PA phosphatases exist in yeast: Dpp1p and Lpp1p. These enzymes dephosphorylate LPA and diacylglycerol pyrophosphate (DGPP) in addition to PA and are integral to the vacuole and Golgi membranes respectively (Faulkner et al. 1999; Furneisen and Carman 2000; Han et al. 2001; Han et al. 2004; Huh et al. 2003; Toke et al. 1998a; Toke et al. 1998b; Wu et al. 1996). Dpp1p and Lpp1p control the pools of PA and DGPP in the vacuole and Golgi membranes and their deletion affects the cellular ratio of different PL classes (Toke et al. 1998b), but they do not appear to be involved in de novo PL synthesis (Han et al. 2006).

Beyond the effect of media composition, PL synthesis is also regulated by progression through culture stages: genes under $\mathrm{UAS}_{\mathrm{INO}}$ control are preferentially expressed during exponential phase and repressed during stationary phase (Carman and Henry 1999; Carman and Han 2007; Carman and Han 2009a; Carman and Henry 2007; Chen et al. 2007b). Although the exact mechanisms of this regulation are not as well understood as those derived from nutrient availability, it is clear that both Pah1p and Opi1p are targets for several kinases, including cell cycle dependant kinases, and that their activities are strongly dependent on their phosphorylation patterns (Sreenivas et al. 2001; Chi et al. 2007; Mah et al. 2005; Sreenivas and Carman 2003; Santos-Rosa et al. 2005; Ptacek et al. 2005; Ubersax et al. 2003; O'Hara et al. 2006; Dephoure et al. 2005; Li et al. 2007; Chang

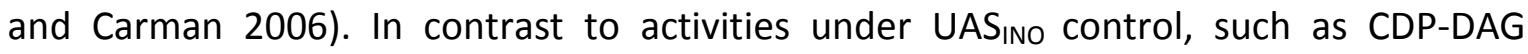
synthase, PS synthase and PE methylation, PGP synthase is preferentially induced during stationary phase (Gaynor et al. 1991), as are PI kinases and PA phosphatases (Hosaka and Yamashita 1984; Holland et al. 1988; Morlock et al. 1988).

\subsection{Phospholipid species composition}

\subsubsection{The acyl-CoA pool}

In the previous section (2.2.) the pathways and mechanisms leading to the formation of different lipid classes were described. As indicated there, alternative pathways exist both 
for synthesis of PA and for synthesis of the major membrane components phosphatidylcholine and phosphatidylethanolamine. The enzymes involved in those pathways have particular acyl chain substrate preferences and, in consequence, provide a certain degree of both specificity and flexibility to $\mathrm{PL}$ species composition in de novo synthesis (Bürgermeister et al. 2004a; Bürgermeister et al. 2004b; Dowd et al. 2001; Zaremberg and McMaster 2002; Zheng and Zou 2001). However, the substrate preferences of the enzymes involved is not sufficient and as an additional parameter the regulation of the acyl-CoA pool composition might be required. The acyl-CoA pool is largely derived from de novo FA synthesis but it also receives major contributions through the activation of free fatty acids (FFA) either produced by lipid deacylation or imported into the cell from the growth media. Depletion of the acyl-CoA pool takes place through lipid synthesis or through FA degradation by $\beta$-oxidation.

Neutral lipid mobilization, in particular, has long been recognized as an important source of FA that can feed into the acyl-CoA pool. Through the last few years it has began to become clear that this function is carefully controlled and that NL mobilization could therefore be not just a source but a regulator of FA availability (Kurat et al. 2009). Furthermore, it also begins to become apparent that NL synthesis is not only relevant in terms of NL production itself, generating a pool for later mobilization, but also through its direct consumption of acyl-CoA acting as a negative regulator of the pool of activated FA. In this regard, NL synthesis provides a protective effect against a disturbance of the acylCoA pool by imported FA (Petschnigg et al. 2009); our own results, which will be presented and discussed through this document, point towards the negative regulation of the acyl-CoA pool by NL synthesis being relevant with regard to endogenously produced FA in addition to imported ones. NL metabolism, however, will not be described in this section, but separately in section 2.5 .

\section{Fatty acid synthesis, elongation and desaturation}

The profile of de novo synthesized fatty acids (FA) in S. cerevisiae is entirely composed of saturated and monounsaturated FA; the most abundant ones being 18:1 (oleic acid), 16:1 (palmitoleic acid), 16:0 (palmitic acid) and 18:0 (stearic acid), followed by minor amounts 
of $14: 0$ (myristic acid). Fatty acids with chains longer than 18 carbons represent, altogether, 1 to $2 \%$ by weight of the total FA content, with 26:0 being the main one within them (Welch and Burlingame 1973; Cottrell et al. 1986).

The necessary preceding step of both FA synthesis and FA elongation is the carboxylation of acetyl-CoA to produce malonyl-CoA (figure 2).

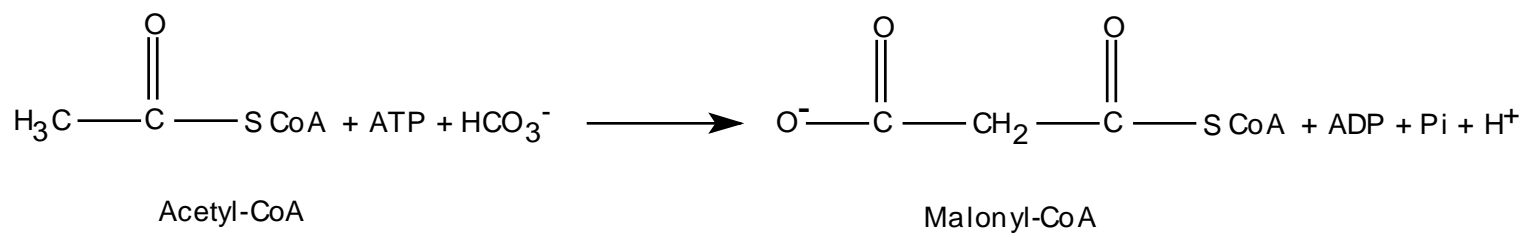

Figure 2. Acetyl-CoA carboxylation.

This reaction is primarily cytosolic, catalyzed by the acetyl-CoA carboxylase encoded by the gene ACC1, also termed FAS3 (Hasslacher et al. 1993; Al-Feel et al. 1992). However, a second acetyl-CoA carboxylase, encoded by the gene HFA1, is present in the mitochondria (Kearsey 1993). Deletion of ACC1 is lethal, even in presence of exogenous fatty acids (Hasslacher et al. 1993), but can be rescued by mislocalization of Hfa1p to the cytosol (Hoja et al. 2004). Deletion of HFA1, in contrast, is not lethal but results in mitochondrial malfunction and the cells' inability to grow on non-fermentable carbon sources (Hiltunen et al. 2005; Hoja et al. 2004).

FA synthesis takes place through condensation of an activated acyl molecule on an activated malonyl molecule (figure 3). Initially, an acetyl residue is transferred from acetyl-CoA to a cysteine residue in the ketoacyl synthase (KS) domain of the Fatty Acid Synthase (FAS) complex, preserving the high transfer potential of the thioester bond. In parallel, malonate is transferred from malonyl-CoA to the $\mathrm{SH}$-residue of the pantetheine cofactor, which is covalently attached to the acyl carrier protein (ACP), again conserving the thioester bond. The acetate molecule on KS is then condensed on malonyl-ACP yielding acetoacetyl-ACP and leaving the KS domain free. Acetoacetyl-ACP is reduced on the expense of NADPH, dehydrated and further reduced to butyryl-ACP. The nascent acyl chain, a butyrate at this stage, is then transferred from ACP to the KS domain and the 
ACP is occupied by a new malonyl residue from malonyl-CoA. The process is then repeated, now with acetate replaced by butyrate and by a longer acyl chain after every round. In the final step, the newly synthesized acyl chain is transferred from the intrinsic ACP to CoA.

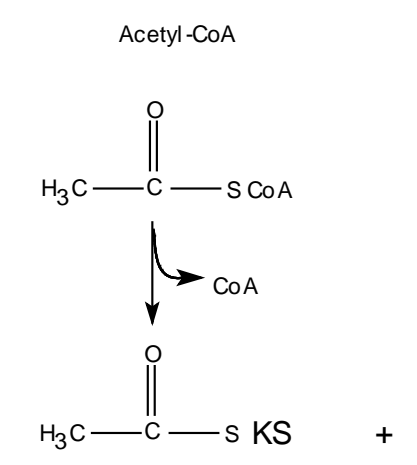

Acetyl thioester on KS domain
Malonyl-CoA<smiles>O=C([O-])CC(=O)S(=O)(=O)[O-]</smiles><smiles>CC1C(C(=O)O)C1C(=O)CC(=O)SC(=O)F</smiles>
Acetoacetyl-ACP

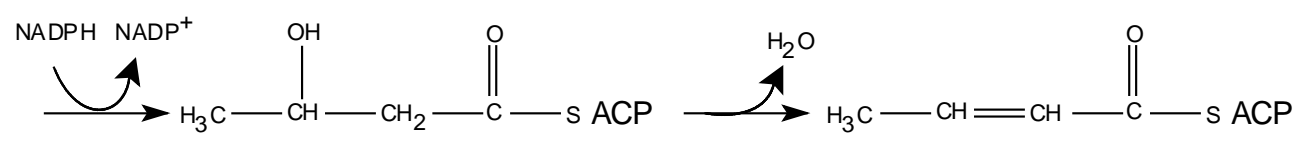

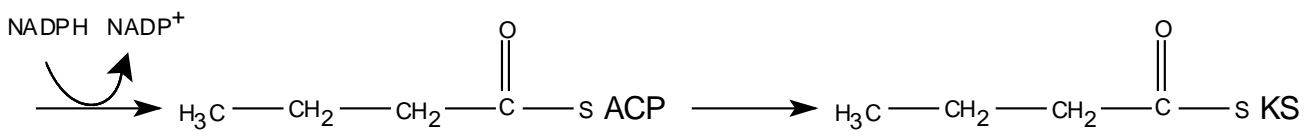

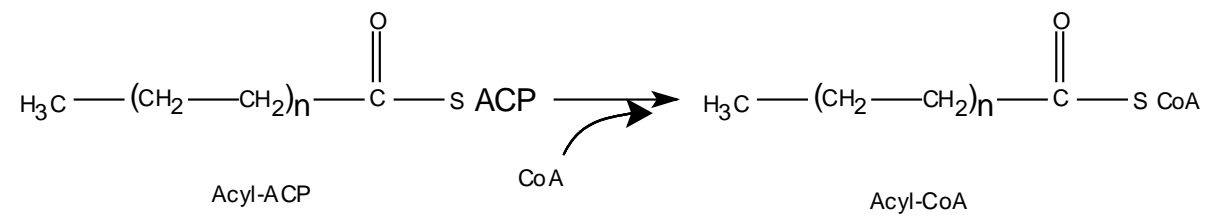

Figure 3. Fatty acid synthesis. Details are described in the main text. The decarboxylation of malonyl-ACP makes the reaction thermodynamically favorable, indirectly linking ATP hydrolysis (required for acetyl-CoA carboxylation, see figure 2) to fatty acid synthesis. The final product of the reaction series, in yeast, is not a free fatty acid but an acyl-CoA molecule.

As with the carboxylation of acetyl-CoA, two parallel systems for FA synthesis exist in the cytosol and the mitochondria. In the cytosolic process, the series of reactions are performed by a type I FAS complex composed of $\alpha$ - and $\beta$-subunits, respectively encoded 
by the genes FAS2 and FAS1, in a hexadimeric arrangement ( $\alpha 6 \beta 6)$. Subunit $\alpha$ contains the $\beta$-ketoacyl synthase, $\beta$-ketoacyl reductase and acyl-carrier protein activities (Mohamed et al. 1988; Schweizer et al. 1984); subunit $\beta$ possesses acetyl-, malonyl- and palmitoyl-transferase activities, as well as dehydratase and enoyl-reductase activities (Schweizer et al. 1984; Schweizer et al. 1986; Chirala et al. 1987). During the condensation, reduction and dehydration reactions, the acyl intermediates are bound to ACP. However, as the ACP is an integral part of the FAS complex, no acyl-ACP is released. In contrast to mammalian FA synthesis, where the final step is mediated by a thioesterase releasing a free fatty acid (FFA), the final step of FA synthesis in yeast is the transfer of the acyl chain from ACP to CoA, releasing acyl-CoA (Lynen et al. 1964; Schweizer et al. 1970). The mitochondrial FAS system is a type II system, with the various enzymatic activities residing in independent polypeptides: Acp1p, acyl-carrier protein (ACP); Cem1p, $\beta$-ketoacyl-ACP synthase; Oar1p, 3-oxoacyl-ACP reductase; Htd2p, 3hydroxyacyl-thioester dehydratase; Etr1p, enoyl-ACP reductase. Additionally, Ppt2p is required for the attachment of the phosphopantetheine prosthetic group to the apoACP (Harington et al. 1993; Kastaniotis et al. 2004; Stuible et al. 1998; Torkko et al. 2001; Schneider et al. 1997; Schneider et al. 1995; Brody et al. 1997). The cytosolic FAS complex is undoubtedly the main one responsible for de novo FA synthesis, cells deficient for it remain viable only if the major fatty acids (14-, 16- and 18-carbon chains) are available in the medium (Schweizer and Bolling 1970). In fact, the mitochondrial system was often referred to as a putative FAS system, although recent reviews consider it a proper FAS system (Tehlivets et al. 2007). The main role of mitochondrial FA synthesis appears to be the production of octanoic acid as a precursor for lipoic acid. Its deficiency leads to respiratory malfunction but does not compromise cell viability ((Tehlivets et al. 2007) and references therein).

Regulation of FA synthesis occurs both at the level of acetyl-CoA carboxylation and of the

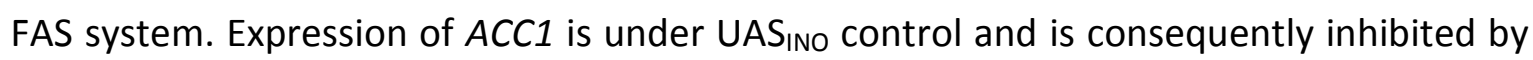
inositol (Carman and Henry 1999; Hasslacher et al. 1993; Chirala et al. 1994), but is also subjected to cell cycle dependant regulation with maximum expression during early G1 phase (Cho et al. 1998). Acc1p activity is down regulated through phosphorylation by the kinase Snf1p (Shirra et al. 2001). The way in which Snf1p activity is regulated has not 
been established; however, the mammalian ortholog of Snf1p, AMP-activated protein kinase, is regulated by the cellular level of acyl-CoA (Taylor et al. 2005) and a similar situation in yeast appears likely (Tehlivets et al. 2007).

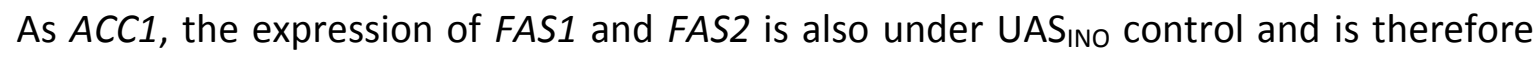
coordinated with PL synthesis through responsiveness to PA levels, inositol and choline availability (Schüller et al. 1992; Chirala 1992; Bar-Joseph et al. 2003). FAS1 and FAS2 expression is inhibited by long-chain FA. This inhibition has been proposed to depend on a UAS FAS element in their promoters, but the actual mechanism is not known (Chirala 1992). The cell cycle dependant expression of FAS1 and FAS2 reaches its maximum during the transition from M to G1 phase (Spellman et al. 1998) and is dependent on histone phosphorylation and acetylation (Kristjuhan et al. 2002; Huisinga and Pugh 2004; Palecek et al. 2002). The most common end product of FA synthesis is 16:0, but shorter and longer FA are also produced (8:0 to 18:0, in vitro 20:0 can also be produced by the FAS system). The factors determining the length of the FA produced by the FAS system have not yet been fully determined. It is however clear, that malonyl-CoA availability, and therefore Acc1p activity, is one of them (Tehlivets et al. 2007; Black and DiRusso 2007).

Like FA synthesis, FA elongation depends on malonyl-CoA and NADPH (Dittrich et al. 1998). However, in contrast to synthesis which is mainly cytosolic, FA elongation takes place primarily at the ER, and secondarily in the mitochondria (Toke and Martin 1996; Dittrich et al. 1998; Bessoule et al. 1988). The condensation reaction in FA elongation can be driven by three different enzymes: Elo1p, Elo2p (standard name Fen1p) and Elo3p (standard name Sur4p). Elo1p is specific for medium and long chain FA, mainly converting 12- and 14-carbon chains to 14- and 16-carbons, although it can also accept 10 carbon chains as substrate (Toke and Martin 1996; Dittrich et al. 1998; Schneiter et al. 2000). Elo2p drives the elongation of FA up to 22 or 24 carbons (Oh et al. 1997) and Elo3p up to 26 carbons (Rössler et al. 2003). The 3-ketoacyl-CoA and enoyl-CoA reductase activities of FA elongation are respectively carried out by Ifa38p and Tsc13p (Beaudoin et al. 2002; Han et al. 2002; Kohlwein et al. 2001); the protein responsible for the dehydration of the intermediate 3-hydroxy acyl-CoA has not been identified. The transcription of Elo1p, Elo2p and Elo3p is down-regulated during stationary phase or under conditions of 
nitrogen starvation; the expression of Elo1p is induced by the presence of 14:0 FA and repressed by 16:0 FA (Gasch et al. 2000; Toke and Martin 1996).

Desaturation of fatty acids takes place in the ER and is driven by a $\Delta 9$-desaturase encoded by the gene OLE1. The major substrates for desaturation are 16:0 and 18:0, but 14:0 can also be utilized. Only monounsaturated fatty acids are produced (Mitchell and Martin 1995; Stukey et al. 1989; Stukey et al. 1990). Expression of OLE1 is highly regulated and responds to a large variety of conditions including carbon source, fatty acids, metal ions, oxygen levels and growth temperature (reviewed in (Martin et al. 2007)). Of particular relevance in the context of the acyl-CoA pool composition and its effect on PL synthesis, is the fact that presence of $16: 0$ or 18:0 in the media induces the expression of OLE1 and does not result in a considerable variation of lipid species distribution, while presence of $16: 1,18: 1$ or non-native unsaturated FA causes a repression of OLE1 and leads to incorporation of the supplemented FA at the expense of other unsaturated species (Bossie and Martin 1989).

\section{Fatty acid import}

Yeast, as many other organisms, has the ability to acquire free fatty acids from the medium and employ them in catabolic and anabolic processes. In fact, given that the glyoxylate cycle is active in yeast, the cells are capable of living on fatty acids as sole carbon source (van Roermund et al. 1995). Import of FA proceeds both through diffusion and through active protein mediated processes (Hamilton 1998; Hamilton and Kamp 1999; Abumrad et al. 1999; Berk and Stump 1999). One active mechanism for FA import, known as vectorial acylation, links FA translocation across the membrane to FA activation (Black and DiRusso 2003). It seems clear that the presence of active acyl-CoA synthetases (ACS) enhances FA uptake into the cells (DiRusso and Black 1999); however, it is also clear that active FA uptake can proceed independently of activation in absence of all ACS activity (Knoll et al. 1995; Scharnewski et al. 2008). FA uptake in yeast, and other eukaryotes, does not appear to be regulated or at least not tightly regulated, as unnatural FA are imported and incorporated into lipids, even displacing natural FA (Bossie and Martin 1989), and, furthermore, as an excessive supply of exogenous FA can 
lead to cell death (Garbarino and Sturley 2009; Petschnigg et al. 2009; Listenberger et al. 2003).

\section{Fatty acid activation}

FFA derived from FA import or released from lipids can be utilized for lipid synthesis, protein modification or energy production through $\beta$-oxidation only after being activated by conversion into acyl-CoA. Acyl-CoA synthesis is a two step process where the energy derived from ATP hydrolysis (and further hydrolysis of pyrophosphate to orthophosphate) is used for the formation of the high transfer potential thioester bond between the FA and CoA (figure 4).

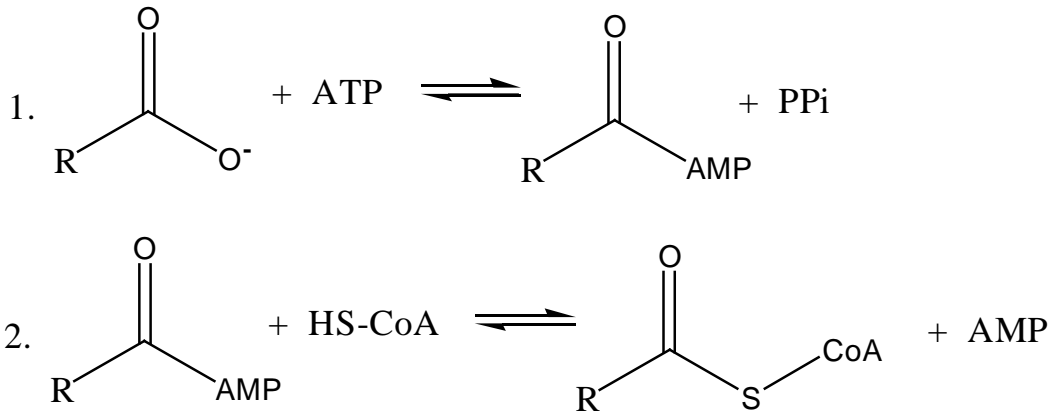

Figure 4. Activation of fatty acids. On the first step the fatty acid is transformed into an acyl-adenylate, on the second step the acyl-adenylate is converted into acyl-CoA

Five genes in yeast code for proteins with ACS activity: FAA1, FAA2, FAA3, FAA4 and FAT1. FAT2 shares some of the structural and functional characteristics of ACS enzymes, within them the AMP binding motif, and is therefore commonly considered alongside with them; however, it does not share other important characteristics of ACS enzymes such as some of the key amino acid residues in the fatty acyl-CoA synthase (FACS) motif (Black and DiRusso 2007). Its enzymatic activity has not been identified and it has been proposed that its AMP binding activity could apply on a molecule other than a FA (Blobel and Erdmann 1996). 
FAA1 and FAA4 encode acyl-CoA synthetases involved in the activation of imported fatty acids (Johnson et al. 1994a; Duronio et al. 1992) and in the recycling of FA derived from lipid deacylation (Scharnewski et al. 2008). They have a preference for substrates with chains from 12- to 16-carbons (Knoll et al. 1994) but can utilize chains from 8- to 20-carbons (Black and DiRusso 2007). Under standard conditions, Faa1p is responsible for most long chain ACS activity in the cells (Faergeman et al. 2001; Duronio et al. 1992), but its functions and those of Faa4p overlap and lack of either one can be compensated by the activity of the other (Knoll et al. 1995). Simultaneous deletion of both genes reduces ACS activity towards 14 - and 16-carbon chains by $99 \%$, makes the rescue of FAS deficient cells by provision of exogenous FA unviable (Knoll et al. 1995) and leads to the accumulation of large amounts of endogenously produced FFA (Scharnewski et al. 2008). Faa1p has been localized on the plasma membrane, ER, LP and mitochondria, while Faa4p has been localized on the ER and LP (Athenstaedt et al. 1999b; Natter et al. 2005; Sickmann et al. 2003; Zou et al. 2003). They appear to be membrane associated rather than membrane bound and they have also been identified as cytosolic proteins (Black and DiRusso 2007).

FAT1 was initially identified by its homology to mammalian fatty acid transport proteins (Schaffer and Lodish 1994; Faergeman et al. 1997) and plays a central role in FA transport within the theory of vectorial acylation (Zou et al. 2003); however, it is not indispensable for fatty acid intake (Scharnewski et al. 2008). Fat1p has been shown to be a very long chain ACS with preference for fatty acids longer than 22 carbons required for maintenance of very long chain fatty acids homeostasis (Choi and Martin 1999; Watkins et al. 1998). It is localized on the ER and peroxisomal membranes as well as on LP (Choi and Martin 1999; Athenstaedt et al. 1999b; Natter et al. 2005). This localization put its role in FA transport across the plasma membrane into question.

FAA2 encodes an ACS (Johnson et al. 1994b) which localizes to the matrix side of peroxisomal membranes and is involved in activation of fatty acids for $\beta$-oxidation (van Roermund et al. 2000; Hettema et al. 1996). It presents highest activity towards fatty acids with chains within 9- and 13-carbons, but accepts a broader range of substrates (Knoll et al. 1994). 
FAA3 encodes an ACS of relatively low activity. Its preferred substrates are fatty acids with chains from 16- to 18-carbons, but can also activate very long chain fatty acids (Johnson et al. 1994b; Knoll et al. 1994). Faa3p is localized on the plasma membrane (Natter et al. 2005) and, as in the case of Fat2p, its biological role remains unknown.

\section{B-oxidation}

In yeast, no mitochondrial $\beta$-oxidation takes place, making oxidative degradation of FA an entirely peroxisomal process (Kunau et al. 1988). Both acyl-CoAs and FFA can be imported into the peroxisome. In the first case, an ATP dependant transport system, composed of at least Pxa1p and Pxa2p, translocates long chain acyl-CoAs into the lumen (Swartzman et al. 1996; Hettema et al. 1996; Hettema and Tabak 2000; Shani and Valle 1996). In the second case, an independent, so far unidentified, mechanism transports non activated medium chain fatty acids into the peroxisome, where they are subsequently esterified to coenzyme A by Faa2p (Hettema et al. 1996; Choi and Martin 1999). Although the two mechanisms show different preferences for long or medium chains, they are not completely excluding: Disruption of PXA1 and PXA2 impairs oxidation of long chain fatty acids, but the process is entirely suppressed only if FAA2 is disrupted as well (Hettema and Tabak 2000). Equivalently, mislocalization of Faa2p to the cytosol, instead of the peroxisomal lumen, makes degradation of medium-chain fatty acids dependant on the import by Pxa1p/Pxa2p (Hettema et al. 1996). Once inside the peroxisome, acyl-CoA is degraded to acetyl-CoA which can either be further oxidized through the citric acid cycle or used for carbohydrate synthesis through the glyoxylate cycle. A description of the reactions and enzymes involved in $\beta$-oxidation can be found for example in (Hiltunen et al. 2003). The role of $\beta$-oxidation in the production of energy and metabolites for anabolic reactions, particularly when FA imported from the medium or derived from NL mobilization during stationary phase constitute the main or sole carbon source, is well established. It is apparent, however, that the function of oxidative FA degradation is not exclusively the production of energy, but also detoxification. Lockshon and coworkers (Lockshon et al. 2007) have shown that growth inhibition of some peroxisomal and $\beta$-oxidation deficient strains in media containing oleate, is not mainly derived from their inability to feed on this FA but rather from the uncontrolled 
incorporation of the FA into membrane lipids. This implies that the depletion of the acylCoA pool by $\beta$-oxidation is not only a secondary effect of FA utilization for energy and metabolite production, but could be, at least under some conditions, a purpose on its own. The observations of Lockshon and coworkers refer to the modulation of acyl-CoA derived from imported FA; whether $\beta$-oxidation plays as well a role in the regulation of the acyl-CoA pool derived from endogenous FA as a purpose on its own, has not been established.

\subsubsection{Phospholipid remodeling}

It is a well established fact that, despite the existence of alternative pathways for PL synthesis and of mechanisms to modulate the species composition of the acyl-CoA pool, de novo PL synthesis is not enough to account for the actual lipid composition of eukaryotic cells (Lands 1958; Lands 1960).

Even under steady culture conditions (e.g. temperature) and in absence of an artificial perturbation of the acyl-CoA pool or of any interference with the biosynthetic machinery, extensive lipid remodeling takes place and is essential for attaining and maintaining lipid homeostasis. Acyl-chain remodeling of phospholipids requires two reactions: deacylation of the lipid and reacylation by acyltransferase or transacylase activity. In S. cerevisiae, acyl-CoA dependant acylation of both sn-1- and sn-2-acyl-glycero-phosphocholine was first demonstrated three decades ago (Yamada et al. 1977); since then it has become clear that deacylation and incorporation of a new acyl chain into the resulting lysolipid occurs not only for PC but for all major phospholipids and that the process is of remarkable importance to the formation of the steady state lipid profile (de Kroon 2007; Boumann et al. 2003; Richard and McMaster 1998; Wagner and Paltauf 1994). This could be partly derived from insufficient specificity and/or flexibility in species composition during de novo synthesis, either due to an insufficient ability to generate the required species distribution within the pools of biosynthetic intermediaries or due to an insufficient ability to partition these precursor and intermediary pools according to species distribution prior to class establishment. Alternatively, or additionally, the 
remodeling process itself, rather than only its outcome, could be required as a part of membrane maturation and transit.

In recent years substantial advance has been achieved towards understanding the reacylation step of lipid remodeling. The acyl-CoA dependant LPAAT enzymes responsible for PA formation during de novo lipid synthesis have been shown to possess more general LPLAT activity. SIc1p acylates lysoPI and lysoPS (Benghezal et al. 2007), while Ale1p covers a wide substrate range: lysoPC, lysoPE, lysoPI, lysoPS and lysoPG (Benghezal et al. 2007; Chen et al. 2007a; Jain et al. 2007; Riekhof et al. 2007; Tamaki et al. 2007). Gup1p is now known to introduce the 26:0 FA in the sn-2 position of PI during GPI anchor maturation (Bosson et al. 2006) and Taz1p has been identified as an acyl-CoA independent acyl-transferase involved in cardiolipin (CL) remodeling (Testet et al. 2005; Xu et al. 2006; Xu et al. 2003; Gu et al. 2004). Furthermore, it has been shown that the fully deacylated species glycerophosphocholine (GPC) and glycerophosphoethanolamine (GPE) can act as acyl acceptors in yeast, although the enzyme or enzymes involved have not been identified (Stålberg et al. 2008).

The deacylation step, in contrast, remains obscure. This reaction is expected to follow a phospholipase A- or B- like mechanism (Yamashita et al. 1997; de Kroon 2007) but, in yeast, clear evidence for the involvement of particular lipases in the process is extremely scarce: The role of Cld1p in the remodeling of cardiolipin is perhaps the only fully established case (Beranek et al. 2009). Besides this, Per1p is required for the deacylation of the GPI anchor although it is not yet clear whether Per1p is the lipase itself (Fujita et al. 2006). For the major phospholipids (PC, PE, PS and PI) the spectra of known phospholipases A or B that could mediate remodeling is not very wide. Four phospholipases B (PLB) have been identified in S. cerevisiae, however their involvement in PL remodeling has not been demonstrated: Plb1p, mainly active against PC and PE; Plb2p, with highest in vitro activity against PS and PE and lower activity towards PI and PC; Plb3p, known to degrade PI (Fyrst et al. 1999; Lee et al. 1994; Merkel et al. 1999; Merkel et al. 2005a) and Nte1p, a lipase with preference for PC derived from the CDPcholine pathway (Zaccheo et al. 2004; Dowd et al. 2001). These proteins possess lysophospholipase in addition to PLB activity. Known Phospholipases A are even scarcer. Phospholipases A activity in the mitochondria has been reported (Bradshaw et al. 2001; 
Yost et al. 1991), but specific proteins were not linked to it then. It is not clear whether Cld1p, the recently identified mitochondrial PLA, accounts for those earlier observations, but it seems unlikely. Cld1p has not yet been extensively characterized but so far it has shown preference for 16:0 FA in the sn-2 position of CL (Beranek et al. 2009), while the activities reported by the groups of Yost and Bradshaw were not so specific. Lro1p possesses phospholipase $\mathrm{A}$ activity and is therefore a candidate to mediate PL remodeling, but again, its role in the process has not been demonstrated. The lipase activity of Lro1p is combined with acyltransferase activity, resulting in the withdrawal of an acyl chain from the sn-2 position of PC or PE and its transfer onto DAG forming TAG rather than releasing a FFA (Dahlqvist et al. 2000; Oelkers et al. 2000).

\subsection{The YB526 strain}

Experimentally, the contribution of different processes or proteins to lipid homeostasis, and in particular to PL remodeling, is most commonly addressed through evaluation of the resulting lipid species. The standard approaches include analysis of changes in steady state lipid profiles upon specific genetic or culture conditions; chasing the incorporation and distribution of an exogenously provided labeled substrate (glycerol backbone, fatty acid and or head group) and following the dilution of label in specific species after interrupted label feeding. These final product approaches provide solid information about acyl chain incorporation and clearly imply a preceding deacylation step, however, deacylation is only indirectly observed. Analysis of the intermediaries is enormously challenging, if not, in some cases, wholly unviable, given that lysoPL and FFA are rapidly processed either by degradation or reacylation.

In the case of FA, metabolization must be preceded by acyl-CoA synthetase mediated activation. Deletion of the genes coding for at least two of these enzymes (FAA1 and FAA4) prevents FA recycling and leads to a very strong accumulation of FFA derived from lipid deacylation (Scharnewski et al. 2008). In the cited study, the introduction of an exogenous desaturase, which exclusively converts lipid-bound 18:1 to $18: 2$, was used as a

marker for the pool of bound FA. The appearance of 18:2 in the pool of FFA, and furthermore the similar ratio of $18: 2$ to other FA in the FFA and bound FA pools, 
indicated that lipid deacylation is in fact the origin of the FFA pool. Without activation the released FFA can neither be reused for lipid synthesis nor be degraded by $\beta$-oxidation; the FFA accumulate in cells and medium and their concentration can be considered as a direct measure of lipid remodeling and lipid degradation. Such mutant strains provide an experimental system in which lipid turnover, PL remodeling and FA homeostasis in general can be analyzed from a new perspective.

In the present study we utilize the acyl-CoA synthetase deficient strain YB526 (YB332faa1 $\Delta$ faa2 $\Delta$ faa3 $\Delta$ faa4 4 ) as a FFA accumulating background. We then proceed by deleting genes of interest on this background and evaluate the impact of those deletions on the pools of free and bound FA as an indication of their involvement in lipid deacylation and FA homeostasis.

\subsection{Neutral lipid metabolism}

The neutral lipids TAG and steryl esters (STE) are commonly used by all kinds of cells as storage compounds, which, upon hydrolysis, constitute a source of DAG, FA and sterols. Their lack of polarity prevents them from forming bilayers or solubilizing in them, neutral lipids are therefore stored separately in the core of lipid particles (Zweytick et al. 2000a).

TAG synthesis utilizes DAG and an acyl chain in either acyl-CoA independent or acyl-CoA dependant reactions. DAG for TAG synthesis is derived from the dephosphorylation of PA, which can be produced either through de novo synthesis as described in section 2.2.2. or through PL degradation by a phospholipase D (McDermott et al. 2004). Additionally, DAG can be directly derived from PL degradation by a phospholipase C (Flick and Thorner 1993), or from TAG degradation as described ahead. Acyl-CoA independent acylation of DAG is catalyzed by the ER-localized enzyme Lro1p, utilizing the sn-2 acyl chain of a PL, preferentially PE and PC (Dahlqvist et al. 2000; Oelkers et al. 2000). AcylCoA dependent DAG acylation is mainly catalyzed by Dga1p, localized on the ER and LP (Sorger and Daum 2002; Sandager et al. 2002; Oelkers et al. 2002; Lehner and Kuksis 1996). In vitro, this enzyme presents preference for 18:1 and 16:0 FA but can also utilize 14:0, 18:0 and FA unnatural to yeast such as 20:4 and 18:2 (Oelkers et al. 2002). The enzymes' ability to use 16:1 was not tested in the cited study. The ER localized enzymes 
Are1p and Are2p are also capable of acylating DAG in an acyl-CoA dependant way; their contribution to TAG synthesis, however, is minimal compared to that of Lro1p and Dga1p (Sorger et al. 2004; Sandager et al. 2002). Lro1p is most active during exponential growth phase and accounts for 65 to $75 \%$ of TAG synthesized during this period; Dga1p, in contrast, is most active during stationary phase, when it mediates up to $50 \%$ of TAG synthesis (Oelkers et al. 2000; Oelkers et al. 2002).

STE formation is accomplished through the esterification of a FA to the hydroxyl group of a sterol. In contrast to other eukaryotes, where the acyl chain can be derived from a PL, in S. cerevisiae this reaction is strictly acyl-CoA dependant. Sterol acylation is mediated by Are1p and Are2p (Yu et al. 1996; Yang et al. 1996). Are2p is the major STE synthase in vivo (Zweytick et al. 2000b) and accounts for 65 to $75 \%$ of total STE synthesis in vitro (Yu et al. 1996). Are2p preferentially utilizes ergosterol as acyl acceptor, but can also acylate ergosterol precursors (Jensen-Pergakes et al. 2001; Polakowski et al. 1999). Are1p, in contrast, while still capable of ergosterol acylation prefers sterol precursors, particularly lanosterol, as acceptors (Zweytick et al. 2000b).

With the exception of Dga1p which is dually localized on the ER and LP, all NL synthesizing enzymes reside in the ER. NL accumulation, however, does not occur on the ER but on LP. Several hypotheses on the mechanisms of LP biogenesis and NL delivery to the LP have been proposed. The currently favored hypothesis indicates that small amounts of newly synthesized NL segregate from other lipids forming hydrophobic micro-droplets between the luminal and cytosolic leaflets of the ER. Once these microdroplets reach a critical size they are engulfed by a monolayer membrane carrying at least a subset of LP resident proteins, and bud from the ER (Reviewed in (Rajakumari et al. 2008)). In the LP TAG remains unorderly packed at the core, followed by several ordered shells of STE and finally by a PL monolayer (Czabany et al. 2008).

Several proteins involved in lipid metabolism reside in the LP (Athenstaedt et al. 1999b); within them are the TAG lipases Tgl3p, Tgl4p and Tgl5p, as well as the STE hydrolases Yeh1p and Tgl1p.

$\operatorname{Tg}|3 p, \operatorname{Tg}| 4 p$ and Tgl5p degrade TAG producing DAG and a FFA; Tgl3p, however, can further hydrolyze DAG. TgI3p is the most active of the TAG lipases and its absence leads 
to increased presence of 14:0, 16:0 and 26:0 in TAG. Absence of Tgl5p increases the content of 26:0, while Tg|4p has a preference for 14:0 and 16:0 (Kurat et al. 2006; Athenstaedt and Daum 2003; Athenstaedt and Daum 2005). Recently, Tgl3p was reported to possess lysoPE specific acyl-CoA dependant LPLAT activity, while acyl-CoA dependant LPAAT activity was found for Tgl5p. The biosynthetic and hydrolytic activities of these proteins rely on different motifs, although within the same domains, and are independent from each other (Rajakumari and Daum 2009). In addition to these three LP localized lipases, Tgl2p, a mitochondrial protein, acts as well as a TAG and probably also as a DAG lipase (van Heusden et al. 1998; Ham et al. 2009).

The LP localized hydrolases Yeh1p and Tgl1p mediate the deacylation of STE leading to the release of a FFA and the sterol molecule. A third STE hydrolase, Yeh2p, is localized on the plasma membrane (Jandrositz et al. 2005; Müllner et al. 2005; Köffel et al. 2005). Under standard conditions Yeh2p is the major STE mobilizing enzyme (Zinser et al. 1993); however, under anaerobic conditions, Yeh1p is responsible for most STE hydrolysis (Köffel and Schneiter 2006).

\subsection{Autophagy}

Autophagy is a process conserved among eukaryotes by which portions of cytoplasm, in some cases including organelles or portions of organelles, are transported to the vacuolar/lysosomal lumen. Two main forms of autophagy exist in yeast: micro- and macro- autophagy (He and Klionsky 2009; Klionsky 2005). Other eukaryotes, mammals in particular, count with a third form: chaperone-mediated autophagy, in which proteins are directly translocated into the lumen of the lysosome (Massey et al. 2004). Micro- and macroautophagy involve large membrane rearrangements, and, as will be seen through the results and discussion in this document, play a prominent role in lipid and FA homeostasis.

Autophagy is most common and best characterized as a response to starvation where the degradation of cytoplasmic content constitutes a source of nutrients (Kuma et al. 2004; Tsukada and Ohsumi 1993; Mizushima et al. 2004). However, it also occurs under nutrient rich conditions as a mechanism to maintain organelle homeostasis, degrade 
damaged or misfolded proteins (Kruse et al. 2006; Kamimoto et al. 2006; Shintani and Klionsky 2004), accomplish cell remodeling during cell differentiation (Levine and Klionsky 2004) and, in mammalian cells, destroy viruses and bacteria (Dorn et al. 2002; Nakagawa et al. 2004; Kirkegaard et al. 2004; Ogawa and Sasakawa 2006; Shintani and Klionsky 2004). Autophagy is also involved in life span extension by caloric restriction (Meléndez et al. 2003; Bergamini et al. 2003; Vellai et al. 2003; Longo and Finch 2003) and in some forms of programmed cell death (Baehrecke 2005). Furthermore, not all forms of autophagy are degradative. A constitutive biosynthetic form, termed cytoplasm to vacuole (Cvt) pathway, delivers vacuole resident hydrolases to their target location (Baba et al. 1997; Scott et al. 1997; Harding et al. 1995).

Macroautophagy and the Cvt pathway are morphologically equivalent and share most of their molecular machinery (Scott et al. 1996; Harding et al. 1996). In this process a double membrane vesicle, termed autophagosome in the degradative pathway and Cvt vesicle in the biosynthetic pathway, encloses the cargo. Formation of the autophagosome begins with a double membrane structure, termed phagophore, which nucleates on the cargo and expands around it, probably by vesicle fusion, eventually enclosing the cargo and fusing into a sealed vesicle (reviewed, for example, in (Yorimitsu and Klionsky 2007a) or (Suzuki and Ohsumi 2007)). The membrane origin of the phagophore remains unknown. In yeast, assembly of the phagophore occurs at a perivacuolar location, termed pre-autophagosomal structure (PAS), where almost all proteins necessary for phagophore nucleation and autophagosome formation are recruited. In mammalian cells a PAS or an equivalent unique structure for phagophore formation has not been identified and might not exist (Suzuki and Ohsumi 2007; Suzuki et al. 2007; He and Greenberg 2004; Xie and Klionsky 2007). The autophagosome is then transported to the vacuole, its outer membrane fuses with the vacuolar membrane and the vesicle formed by the inner membrane, termed autophagic body or Cvt body, is released into the vacuolar lumen where it, and its cargo, are degraded (Klionsky 2005).

Microautophagy proceeds through a direct invagination of the vacuolar membrane around a portion of cytosol, in some cases including an organelle or part of it; the invagination is sealed and buds into the lumen of the vacuole where it is degraded. Although this process is also common in S. cerevisiae it has been more thoroughly 
studied in other organisms (Klionsky 2005; Klionsky et al. 2007; Farré and Subramani 2004; Müller et al. 2000; Sattler and Mayer 2000).

In some cases autophagy can turn over random bulk portions of cytosol, in other cases, however, cargo selection is specific. Specific cargo selection includes biosynthetic material in the Cvt pathway, ubiquitinated protein aggregates and organelles (Kraft et al. 2009; van der Klei et al. 2009). A large range of organelles can be degraded through autophagy, in each case the process receives a specific name according to the organelle transported: Mitochondria, mitophagy (Kim et al. 2007); ribosomes, ribophagy (Kraft et al. 2008); peroxisomes, pexophagy (Dunn et al. 2005); Nuclear envelope, nucleophagy (Dwivedi and Ahnn 2009) or more commonly piecemeal autophagy of the nucleus (Roberts et al. 2003; Krick et al. 2008); LP (or lipid droplet), lipophagy (Singh et al. 2009); ER, reticulophagy or ERphagy (Bernales et al. 2006; Bernales et al. 2007); chloroplast, chlorophagy (Hayward et al. 2009). A similar terminology is sometimes used for the autophagic sequestration of protein aggregates, aggrephagy ( $\varnothing$ verbye et al. 2007; Iwata et al. 2005), or pathogens, xenophagy (Levine 2005). In some cases this terminology is modified to indicate whether the process is micro- or macroautophagic, for example: micropexophagy, macropexophagy, piecemeal microautophagy of the nucleus.

\subsection{Scope of this work}

As indicated in the preceding sections, the regulation of PL species composition, in which $\mathrm{PL}$ deacylation plays a fundamental role, is an essential element within lipid metabolism. The central purpose of the present work is the identification of enzymes and pathways responsible for PL deacylation in S. cerevisiae. Given that PL deacylation is intrinsically related to FA trafficking in general, we do not restrict ourselves to the analysis of deacylation as an isolated event, but, as a secondary purpose of the work, pursue to contextualize our findings within the broader phenomena of PL and FA homeostasis. 



\section{Materials and Methods}

\subsection{Materials}

\subsubsection{Chemical Reagents}

All chemicals were of analytical grade and purchased from Amersham Bioscience (Freiburg), Duchefa (Haarlem, Netherlands), Macherey-Nagel (Düren), Promega (Madison, USA), Roth (Karlsruhe), Serva (Heidelberg) or Sigma-Aldrich (Steinheim) unless otherwise indicated.

1-Ethyl-3-(3-Dimethyl-aminopropyl)carbodiimid (EDAC)

1-Palmitoyl-2-Hydroxy-sn-Glycero-3-Phosphoethanolamine

5-Fluoro-Orotic Acid (5-FOA)

Complete Supplement Media (CSM)

with or without Uracil, Leucine, Histidine

Geneticin G418

Nonidet P40

Nourseothricin

Phleomycin

SeaKem LE Agarose

Yeast Nitrogen Base (YNB) with Ammonium Sulphate
Fluka Biochemika (Steinheim)

Avanti Polar Lipids (Alabaster, USA)

Zymo Research (Orange, USA)

Q-BIOgen (Heidelberg)

Gibco (Paisly, UK)

Fluka Biochemika (Steinheim)

WERNER BioAgents (Jena)

InvivoGen (Toulouse, France)

Cambrex (Rockland, USA)

MP Biomedicals (Illkirch, France)

\subsubsection{Molecular Biology Reagents}

Ex Taq DNA polymerase

Generuler Ladder Mix

Master pure yeast DNA purification kit

Mssl Restriction endonuclease

Nucleospin Extract II

Phusion High-Fidelity PCR Kit
TaKaRa Bio Inc. (Verviers, Belgium)

Fermentas (St. Leon-Roth)

Epicenter Biotechnologies (Oldendorf)

Fermentas (St. Leon-Roth)

Macherey-Nagel (Düren)

Finnzymes (Espoo, Finland) 


\subsubsection{Cell Culture Media}

YPD:

$10 \mathrm{~g}$ Yeast extract

20 g Peptone

20 g Dextrose

$1 \mathrm{~L}$ Distilled water

Sterilized by autoclaving $20 \mathrm{~min}$ at $120^{\circ} \mathrm{C}$.

YPR:

10 g Yeast extract

20 g Peptone

$950 \mathrm{ml}$ Distilled water

Sterilized by autoclaving $20 \mathrm{~min}$ at $120^{\circ} \mathrm{C}$

$20 \mathrm{~g}$ Raffinose in $50 \mathrm{ml}$ distilled water. Sterilized by filtration and added to the medium after cooling.

\section{Synthetic Defined Media (SD):}

$6.7 \mathrm{~g}$ YNB with ammonium sulfate, without amino acids

Either 0.79 g complete CSM, 0.77 g CSM without uracil, 0.69 g CSM without leucine or $0.77 \mathrm{~g}$ CSM without histidine

$950 \mathrm{ml}$ distilled water

Sterilized by autoclaving $20 \mathrm{~min}$ at $120^{\circ} \mathrm{C}$

$20 \mathrm{~g}$ either raffinose or dextrose in $50 \mathrm{ml}$ distilled water. Sterilized by filtration and added to the medium after cooling.

Handling of $p s d 1 \Delta p s d 2 \Delta$ strains in SD media requires additional $2 \mathrm{mM}$ ethanolamine.

YPD or SD Plates: $\quad 20 \mathrm{~g}$ bacto agar added to the medium before autoclaving. 
Antibiotic selective plates: Final concentration in the media:

$$
\begin{array}{ll}
\text { Hygromycin: } & 300 \mu \mathrm{g} / \mathrm{ml} . \\
\text { Nourseothricin: } & 100 \mu \mathrm{g} / \mathrm{ml} \\
\text { Geneticin: } & 300 \mu \mathrm{g} / \mathrm{ml} \\
\text { Phleomycin: } & 12 \mu \mathrm{g} / \mathrm{ml}
\end{array}
$$

Antibiotics were added after sterilization and cooling of the media to approximately $60^{\circ} \mathrm{C}$.

5-FOA plates:

5-FOA plates were prepared as SD plates with higher Uracil (final $50 \mathrm{mg} / \mathrm{L}$ ) content and $1 \mathrm{~g} / \mathrm{L} \mathrm{5-FOA}$.

5-FOA was dissolved in water at $37{ }^{\circ} \mathrm{C}$, sterile filtered and added to the media after autoclaving

\subsubsection{Cell Lines}

W303: wild type. MAT a, trp1, his3, ura3, leu2

(Thomas and Rothstein 1989)

MF17: W303 plb1::TRP1, plb2::HIS3, plb3::KANMX4

(Merkel et al. 1999)

YB332: wild type (S288C background). MAT a NMTI, ura3, ade2, lys2, leu2, his3

(Johnson et al. 1994a)

YB524: YB332 faa4::LYS2

(Johnson et al. 1994a)

YB526: YB332 faa1::HIS3, faa2::LEU2, faa3::LEU2, faa4::LYS2

(Johnson et al. 1994a)

MS51: YB526 fat1::BLE

(Scharnewski et al. 2008)

BYP: $\quad$ BY4741 (Mat a; his3, leu2, met15, ura3) plc1::KANMX4

From EUROSCARF

Further strains produced within this work are described in appendix 1. 


\subsection{Methods}

\subsubsection{Cell Stocks}

Rich (YPD) medium - agar plates stored at $4{ }^{\circ} \mathrm{C}$ were used as transient stocks for wild type cells and cells with genome integrated mutations. Synthetic Defined media (SD) agar plates without the corresponding nutrients were used as stocks for cells carrying plasmids. The plates were inoculated by striping the cells with a sterile loop and incubated at $30 \mathrm{C}$ for 2-3 days before storage.

Glycerol Stocks were used for long term storage. $4 \mathrm{ml}$ YPD media in $13 \mathrm{ml}$ sterile plastic tubes were inoculated with cells scraped from agar plates by means of sterile loops. The tubes were closed with pressure fitting caps and the suspensions were incubated for 24 to $30 \mathrm{~h}$ at $30{ }^{\circ} \mathrm{C}$ with shaking at $210 \mathrm{rpm} .900 \mu \mathrm{l}$ of the suspension were transferred to $2 \mathrm{ml}$ cryovials, $900 \mu \mathrm{l} 50 \%(\mathrm{w} / \mathrm{w}$ ) sterile glycerol (aq.) were added and thoroughly mixed by pippeting. The stocks were stored at $-80^{\circ} \mathrm{C}$.

\subsubsection{Genetic Manipulation}

\subsubsection{Culture}

Preculture. $20 \mathrm{ml}$ YPD in sterile $100 \mathrm{ml}$ flasks were inoculated with cells scraped from agar plates by means of sterile loops. The flask's mouths were sealed with aluminum foil and the suspensions incubated over night at $30^{\circ} \mathrm{C}$ with shaking at $210 \mathrm{rpm}$.

Optical density determination. Aliquots of the cultures were diluted with distilled water to a final volume of $1 \mathrm{ml}$. In each case the dilution factor was chosen to obtain a suspension with an absorbance at $600 \mathrm{~nm}$ beneath 1 . The suspensions were centrifuged at $5000 \mathrm{rpm}$ for 2 minutes (Eppendorf 5417R), the supernatants were discarded and the pellets resuspended in $1 \mathrm{ml}$ distilled water. The optical densities (absorbance) at $600 \mathrm{~nm}$ $\left(O D_{600}\right)$ of the resulting suspensions were measured using distilled water as a blank (Amersham Bioscience, Ultraspec 1100 pro). 
Culture. $20 \mathrm{ml}$ YPD medium, in sterile $100 \mathrm{ml}$ flasks, were inoculated with aliquots of the corresponding precultures to an $\mathrm{OD}_{600}$ of 0.4 . The flask's mouths were sealed with aluminum foil and the suspensions incubated at $30{ }^{\circ} \mathrm{C}$ with shaking at $210 \mathrm{rpm}$ until reaching an $\mathrm{OD}_{600}$ within 0.8 and 1.2 (ca. $4 \mathrm{~h}$ ).

\subsubsection{PCR Based Gene Deletion}

Gene deletions were carried out through the method described by Wach and coworkers (Wach et al. 1994).

In this method, a deletion cassette (linear double stranded DNA, with terminal regions homologous to the flanking regions of the open reading frame (ORF) to be deleted, including the start and stop codons), is transformed into the cell. The natural and in yeast highly efficient phenomenon of homologous recombination exchanges the entire ORF for the introduced DNA. In between the homologous regions required for recombination, the deletion cassette carries a selection marker flanked by a promoter and a terminator element. These elements are themselves flanked by 18-19 bases primer binding sites (pbs).

Deletion cassettes were produced by PCR employing template DNA coding for the upstream pbs - promoter - selection marker - terminator - downstream pbs sequence, and primers with a 18-19 bases $3^{\prime}$ region complementary to the $5^{\prime}$ ends of the template (pbs) plus a ca. 45 bases $5^{\prime}$ overhang homologous to the targeted ORF flanking regions (figure 5).

The presence of the pbs adds versatility to the method since it makes the PCR primers specific for the targeted gene, but unspecific towards the selection construct to be used.

In some cases locus of X-over P1 (loxP) sites are additionally present in between the primer binding sites and the promoter or terminator elements:

upstream pbs - loxP - promoter - selection marker - terminator - loxP - downstream pbs

This allows for the selection marker to be withdrawn from the genome at a later stage and reutilized for the deletion of further genes (see section 3.2.2.6.). 


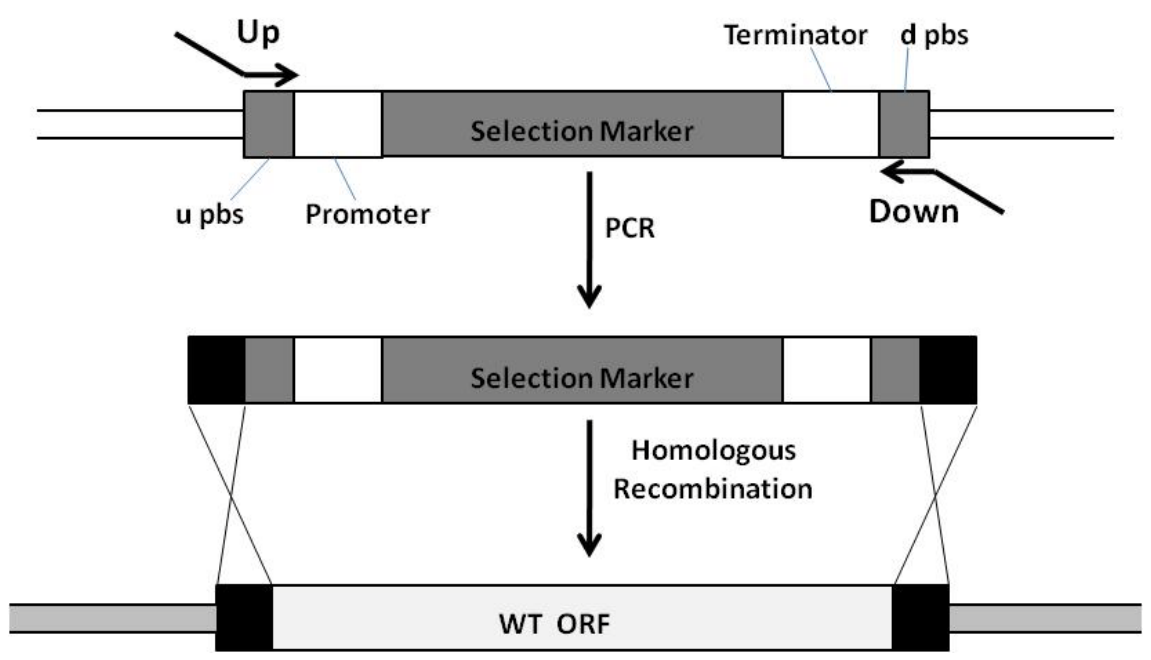

Figure 5. PCR synthesis of deletion cassettes and gene deletion by homologous recombination. UP: forward primer; DOWN: reverse primer; $u$ and d pbs: upstream and downstream primer binding sites; WT ORF: wild type open reading frame

\subsubsection{Synthesis of Deletion Cassettes}

Within this work thirty one different genes were targeted for deletion in different combinations, using a set of 6 resistance markers:

Hygromycin phosphotransferase (HYG) under control of the TEF promoter and the CYC terminator. Confers resistance to the antibiotic hygromycin.

Nourseothricin $\mathrm{N}$-acetyltransferase (NAT) under control of TEF promoter and ADH terminator. Confers resistance to the antibiotic nourseothricin.

Aminoglycoside 3'-phosphotransferase (KanMX4) under control of the TEF promoter and the TEF terminator. Confers resistance to the antibiotic G418.

ble gene from Tn5 (BLE) under control of TEF promoter and the terminator of the URA3 gene from Klyveromyces lactis. Confers resistance to the antibiotic phleomycin.

Orotidine 5-phosphate decarboxylase (URA3) of Klyveromyces lactis driven by its native promoter and terminator. Complements uracil auxotrophy.

Isopropylmalate dehydrogenase (LEU2) of Klyveromyces lactis driven by its native promoter and terminator. Complements leucine auxotrophy. 
The plasmids carrying the selection markers were obtained from EUROSCARF: pAG32 (HYG), pAG25 (NAT) (Goldstein and McCusker 1999); pUG6 (KanMX4) (Güldener et al. 1996); pUG66 (BLE), pUG72 (URA3), pUG73 (LEU2) (Güldener et al. 2002). Modifications to the original plasmids, such as exchange of promoters and terminators were performed by Dr. Martin Fulda.

The targeted gene - marker combination used in each particular case can be seen in appendix 1 , where the genotypes of the resulting strains are described.

Two different sets of PCR conditions were utilized for synthesis of the resistance cassettes. Program 1 was used for cassettes containing the NAT marker, program 2 was used for cassettes with all other markers. The sequences of the primers used for amplification of the resistance cassettes are presented in appendix 2 .

PCR mix:

$\begin{array}{ll}\text { Template DNA }(10 \mathrm{ng} / \mu \mathrm{l}) & 1 \mu \mathrm{l} \\ \text { Phusion Polymerase buffer } 5 \mathrm{x} & 10 \mu \mathrm{l} \\ \text { dNTP mix (2.5 mM each) } & 4 \mu \mathrm{l} \\ \text { Primer up }(10 \mu \mathrm{M}) & 1 \mu \mathrm{l} \\ \text { Primer down }(10 \mu \mathrm{M}) & 1 \mu \mathrm{l} \\ \text { Phusion Polymerase }(2 \mathrm{U} / \mu \mathrm{l}) & 1 \mu \mathrm{l} \\ \text { Distilled water } & 32 \mu \mathrm{l} \\ \mathrm{MgCl}_{2}(50 \mathrm{mM}) & 0.7 \mu \mathrm{l} \text { (only for cassettes with NAT marker) }\end{array}$

PCR program 1:

PCR program 2:
1) $98^{\circ} \mathrm{C}$
$4 \min$
2) $98^{\circ} \mathrm{C}$
$50 \mathrm{sec}$
3) $62.5^{\circ} \mathrm{C}$
$30 \mathrm{sec}$
4) $72{ }^{\circ} \mathrm{C}$
$90 \mathrm{sec}$

1) $98^{\circ} \mathrm{C}$

$4 \min$

2) $98^{\circ} \mathrm{C}$

$20 \mathrm{sec}$

3) $58^{\circ} \mathrm{C}$

$30 \mathrm{sec}$

4) $72^{\circ} \mathrm{C}$

$90 \mathrm{sec}$

steps 2 - 4 repeated 35 times

5) $72^{\circ} \mathrm{C} \quad 5 \mathrm{~min}$

steps 2 - 4 repeated 35 times

5) $72^{\circ} \mathrm{C} \quad 5 \mathrm{~min}$ 
An aliquot of the PCR products $(5 \mu \mathrm{l})$ was analyzed by gel electrophoresis $(1 \%(\mathrm{w} / \mathrm{v})$ agarose gel in TBE buffer 1x. $110 \mathrm{~V}$ for 30-40 min. Visualized and photographed by fluorescence under UV light (312nm) after $15 \mathrm{~min}$ submersion in $0.2 \mathrm{mg} / \mathrm{ml}$ (aq.) ethidium bromide).

For each gene deletion the products of two identical PCR reactions (90 $\mu$ l remaining after gel analysis) were pooled, purified on a GFX column (Macherey Nagel) using $50 \mu \mathrm{l}$ distilled water for elution and utilized for transformation.

TBE buffer 10x:

$\mathrm{pH} 8.3$
$108 \mathrm{~g}$ Tris base

55 g Boric acid

$40 \mathrm{ml} 0.5 \mathrm{M}$ EDTA

Distilled water to $1 \mathrm{~L}$

\subsubsection{Transformation}

The deletion cassettes were transformed into the cells using a modified version of the protocol developed by Ito and coworkers and refined by Gietz and coworkers (Gietz et al. 1992; Ito et al. 1983).

For each transformation to be done, $10 \mathrm{ml}$ of a cell suspension in YPD medium, grown to an optical density between 0.8 and 1.2 (section 3.2.2.1.), were transferred to a $15 \mathrm{ml}$ falcon tube and pelleted by centrifugation at $3000 \mathrm{rpm}$ for $3 \mathrm{~min}$ (Eppendorf $5810 \mathrm{R}$ centrifuge). The supernatant was discarded and the pellet resuspended in $10 \mathrm{ml}$ distilled sterile water. The suspension was again pelleted under the same conditions and the supernatant discarded. The pellet was resuspended in $400 \mu$ L LITE $1 \mathrm{x}$ solution and split into two aliquots of $200 \mu \mathrm{l}$ each, one to be used for transformation and one to be used as control.

To these, now competent, $200 \mu \mathrm{l}$ cell suspension, $30 \mu \mathrm{l}(10 \mu \mathrm{g} / \mu \mathrm{l})$ single stranded salmon sperm carrier DNA* and $50 \mu \mathrm{l}$ deletion cassette DNA were added (note that $50 \mu \mathrm{l}$ purified deletion cassette correspond to $c a .90 \mu \mathrm{l}$ PCR product). For the aliquots to be used as 
controls, $50 \mu \mathrm{l}$ distilled sterile water were added instead of the deletion cassette. $800 \mu \mathrm{l}$ $40 \%(w / v)$ PEG 4000 in LITE 1x were added and the mixture was briefly vortexed.

*Carrier DNA is denatured by boiling during $20 \mathrm{~min}$ and frozen at $-20{ }^{\circ} \mathrm{C}$. Prior to use the DNA solution is thawed at $65^{\circ} \mathrm{C}$ during $5 \mathrm{~min}$

The cells were then incubated for $15 \mathrm{~min}$ at $30^{\circ} \mathrm{C}$, shaken and incubated for another $15 \mathrm{~min}$. After incubation the cells were heat shocked at $42{ }^{\circ} \mathrm{C}$ for $25 \mathrm{~min}$ and centrifuged at $7000 \mathrm{rpm}$ for 30 seconds (Eppendorf 5415R centrifuge). The supernatant was completely removed with a micropipette. If the selection marker encoded a protein conferring resistance to an antibiotic, the pellet was resuspended in $1 \mathrm{ml}$ YPD and incubated at $30{ }^{\circ} \mathrm{C}$ for 2 to 3 hours. If the marker encoded a protein providing auxotrophic complementation, the pellet was resuspended in $1 \mathrm{ml} \mathrm{SD}$ media without the pertinent nutrients and incubated at $30^{\circ} \mathrm{C}$ for 45 to $60 \mathrm{~min}$. In either case the cells were then pelleted by centrifugation at $4000 \mathrm{rpm}$ during $30 \mathrm{sec}$, the SN was discarded and the pellet resuspended in $c a .100 \mu \mathrm{l}$ distilled sterile water. Cell suspensions were then plated on corresponding selective plates.

The plates were allowed to dry for some minutes before being closed and incubated at $30{ }^{\circ} \mathrm{C}$ for $3-5$ days. Single colonies appearing on the plates were picked with a sterile loop and striped on new selective plates. These new plates were incubated at $30{ }^{\circ} \mathrm{C}$ for 2-3 days and then stored at $4{ }^{\circ} \mathrm{C}$. Cells for further work (cultures for DNA isolation, further genetic manipulation or cultures for lipid and fatty acid profiling experiments) were taken from this secondary plates (or plates derived from them) and not from the primary plates where the transformation is initially plated.

LITE 1X:

$0.1 \mathrm{M}$ Lithium acetate

$10 \mathrm{mM}$ TrisHCl, pH 7.5

1 mM EDTA 


\subsubsection{PCR Control of Gene Deletion}

DNA Isolation. $4 \mathrm{ml}$ YPD medium, in sterile $10 \mathrm{ml}$ glass tubes, were inoculated with cells scraped from agar plates by means of sterile loops. The tubes were closed with metal caps and incubated overnight at $30^{\circ} \mathrm{C}$ with shaking at $210 \mathrm{rpm}$.

$2 \mathrm{ml}$ of cell suspension were transferred into $2 \mathrm{ml}$ microfuge tubes and pelleted by centrifugation at $5000 \mathrm{rpm}$ for 2 min (Eppendorf 5415R centrifuge). DNA was extracted and isolated from these cell pellets with "Master pure yeast DNA purification kit" (Epicenter Biotechnologies) according to the producers' instructions. DNA was resuspended in $50 \mu \mathrm{l}$ distilled water, concentrations were determined and dilutions to $500 \mathrm{ng} / \mu \mathrm{l}$ were prepared.

PCR. Genomic DNA was used as template for PCR employing different combinations of primers flanking the targeted ORF, internal to the targeted ORF, and internal to the selection marker (figure 6). The resulting band pattern indicates the presence or absence of both the targeted gene and the employed marker.
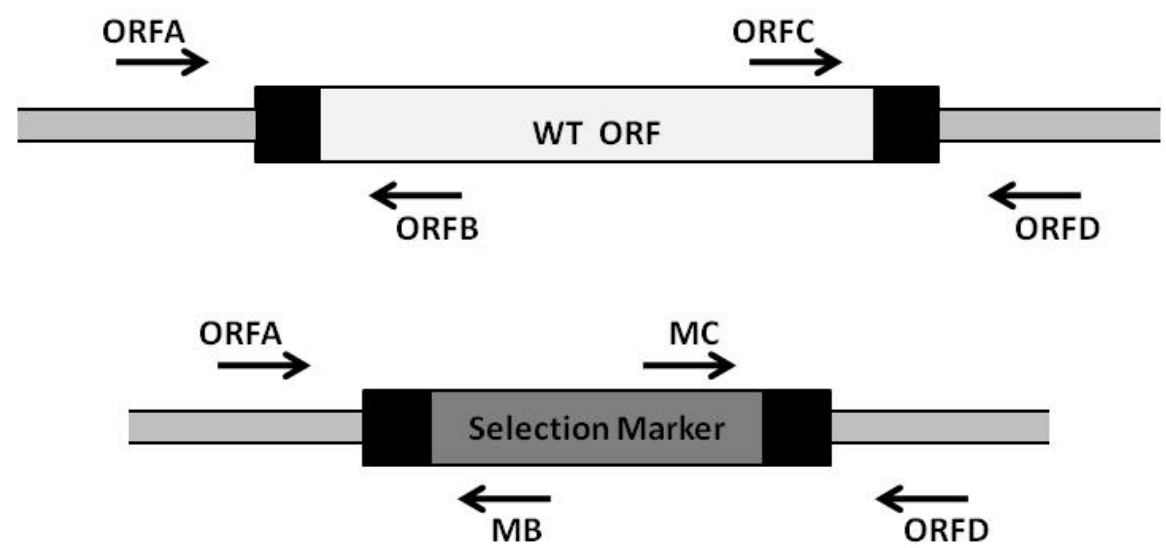

Figure 6. PCR control of gene deletion. For the wild type loci, PCR products are obtained with the ORFA/ORFB and ORFC/ORFD primer combinations. No products are obtained for the ORFA/MB and MC/ORFD combinations. The situation is reversed for the mutated loci.

Primer sequences are presented in appendix 2. 
PCR mix:

\begin{tabular}{|c|c|c|}
\hline Genomic DNA (500 ng/ $/ \mathrm{l})$ & $1 \mu l$ & 1) $94^{\circ} \mathrm{C} 3 \mathrm{~min}$ \\
\hline $\begin{array}{l}\text { Primer forward }(10 \mu \mathrm{M}) \\
\text { ORFA, ORFC or MC }\end{array}$ & $1 \mu l$ & 2) $94^{\circ} \mathrm{C} 30 \mathrm{sec}$ \\
\hline Primer reverse $(10 \mu \mathrm{M})$ & $1 \mu \mathrm{l}$ & 3) $54{ }^{\circ} \mathrm{C} 30 \mathrm{sec}$ \\
\hline ORFB, ORFD or MB & & 4) $72{ }^{\circ} \mathrm{C} 2 \mathrm{~min}$ \\
\hline ExTaq buffer (10x) & $5 \mu l$ & steps $2-4$ repeated 34 times \\
\hline dNTP mix (2.5 mM each) & $4 \mu \mathrm{l}$ & 5) $72^{\circ} \mathrm{C} 5 \mathrm{~min}$ \\
\hline ExTaq polymerase $(5 \mathrm{U} / \mu \mathrm{l})$ & $0.4 \mu \mathrm{l}$ & \\
\hline Distilled water & $37.6 \mu l$ & \\
\hline
\end{tabular}

PCR products where analyzed by gel electrophoresis as described at the end of section 3.2.2.3.

\subsubsection{Recycling of Resistance Markers}

As indicated in section 3.2.2.2. IoxP sites were sometimes included in the deletion cassettes. This allows for the resistance marker to be deleted upon expression of the Cre recombinase (Güldener et al. 2002; Güldener et al. 1996).

A pSH47 expression vector (Güldener et al. 1996), selectable by uracil auxotrophy complementation, which carries the cre ORF under control of a GAL1 promoter, was transformed into the cells as described in sections 3.2.2.1. and 3.2.2.4.

Cells carrying the vector were scraped from SD - ura agar plates by means of a sterile loop and inoculated in $20 \mathrm{ml} \mathrm{SD} \mathrm{-} \mathrm{ura} \mathrm{media} \mathrm{(with} \mathrm{raffinose} \mathrm{as} \mathrm{carbon} \mathrm{source)} \mathrm{in} 100 \mathrm{ml}$ flasks. The cell suspension was incubated for $30 \mathrm{~h}$ at $30{ }^{\circ} \mathrm{C}$ with shaking at $210 \mathrm{rpm} .2 \mathrm{ml}$ $20 \%(\mathrm{w} / \mathrm{v})$ sterile filtered galactose were added to induce expression of the Cre recombinase and the culture was further incubated for 4 to $6 \mathrm{~h} .50 \mu \mathrm{l}$ of cell suspension were plated on YPD agar and incubated at $30^{\circ} \mathrm{C}$ for $2-3$ days. 10 to 20 single colonies were picked and transferred in parallel to YPD agar plates and plates selective for the marker being removed. After $2-3$ days of incubation at $30{ }^{\circ} \mathrm{C}$, cells were picked from YPD agar plates if their counterparts on selective plates had not survived. 
These cells where then cultured for DNA isolation and PCR analysis as described in section 3.2.2.5. In this case the successful removal of the marker was indicated by absence of bands upon primer combinations ORFA/MB and MC/ORFD and a low molecular weight band upon primer combination ORFA/ORFD (figure 6).

Once the removal of the selection marker was verified, the cells were striped on complete SD agar plates with $1 \mathrm{~g} / \mathrm{L}$ 5-fluoro-orotic acid (5-FOA) and high uracil content (50 mg/L) to select for loss of the pSH47 vector. 5-FOA itself is not toxic to yeast; however, in presence of the orotidine 5-phosphate decarboxylase encoded by the URA3 gene, 5-FOA is ultimately converted to 5-fluorodeoxyuridine-monophosphate which inhibits the thymidylate synthase and prevents synthesis of thymine nucleotides (Boeke et al. 1984; Rathod et al. 1992).

After 3 days of incubation at $30{ }^{\circ} \mathrm{C}$, cells were picked from these plates, striped once again on an empty region of the same plate or on a new complete SD 5-FOA agar plate and incubated at $30^{\circ} \mathrm{C}$ for 3 more days.

Cells were then scraped from 5-FOA containing plates, diluted on YPD agar plates and incubated at $30^{\circ} \mathrm{C}$ for $2-3$ days.

Cell dilution on plates is accomplished by dragging the loop across the preceding stripe without scraping new cells from the source plate (figure 7).

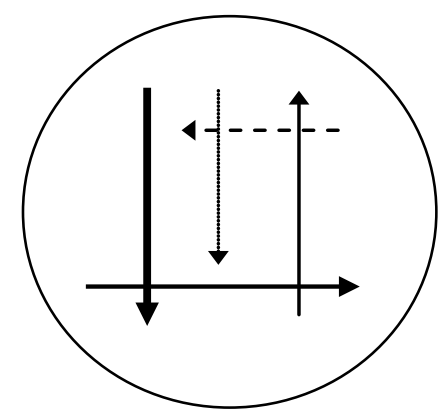

Figure 7. On plate cell dilution. The transfer loop is successively dragged across the preceding stripe. The final stripes result in single colonies.

Single colonies were picked from these plates and transferred in parallel to YPD agar and SD - ura agar plates. After 3 days of incubation at $30{ }^{\circ} \mathrm{C}$ cells were picked from YPD agar 
plates and kept for further work if their counterparts on selective plates had not survived.

\subsubsection{Construction of a Strain Expressing a Heat Degradable PIc1p}

In some genetic backgrounds deletion of PLC1 results in a growth defect. To circumvent this, a strain expressing a recombinant PIc1p which is functional at low temperatures and targeted for degradation at higher temperatures was constructed by fusion of the wild type PLC1 ORF with a heat-inducible degron cassette.

The degron motif is a mutated temperature-sensitive version of the mouse dihydrofolate reductase (DHFR) protein, which, after posttranslational modification, displays an $\mathrm{N}$ terminal arginine residue that acts as binding site for Ubr1p. It also contains several lysine residues that are targets for ubiquitination. At elevated temperatures $\left(37^{\circ} \mathrm{C}\right)$ the protein is partially unfolded exposing the arginine and lysine residues. Binding of Ubr1p promotes ubiquitination and leads to proteasomal degradation of the protein (SanchezDiaz et al. 2004).

The template for synthesis of the degron cassette consisted of a KanMX selection marker followed by a CUP1 (copper inducible) promoter, the degron motif, a c-Myc epitope and a $5 x$ glycine alanine linker in a plasmid (pKL187, EUROSCARF, Sanchez-Diaz et al.2004).

This template was amplified by PCR as described in section 3.2.2.3. with PCR program 2. The upstream (5') primer (DPLC1up) consisted of $50 \mathrm{bp}$ homologous to a region within the PLC1 wild type promoter followed by $20 \mathrm{bp}$ corresponding to the beginning of the cassettes' template; the downstream (3') primer (DPLC1do) was the sequence antiparallel to the last $20 \mathrm{bp}$ of the template followed by the first $50 \mathrm{bp}$ (including the start codon) of the PLC1 ORF (figure 8).

The sequence of the primers is presented in appendix 2. 


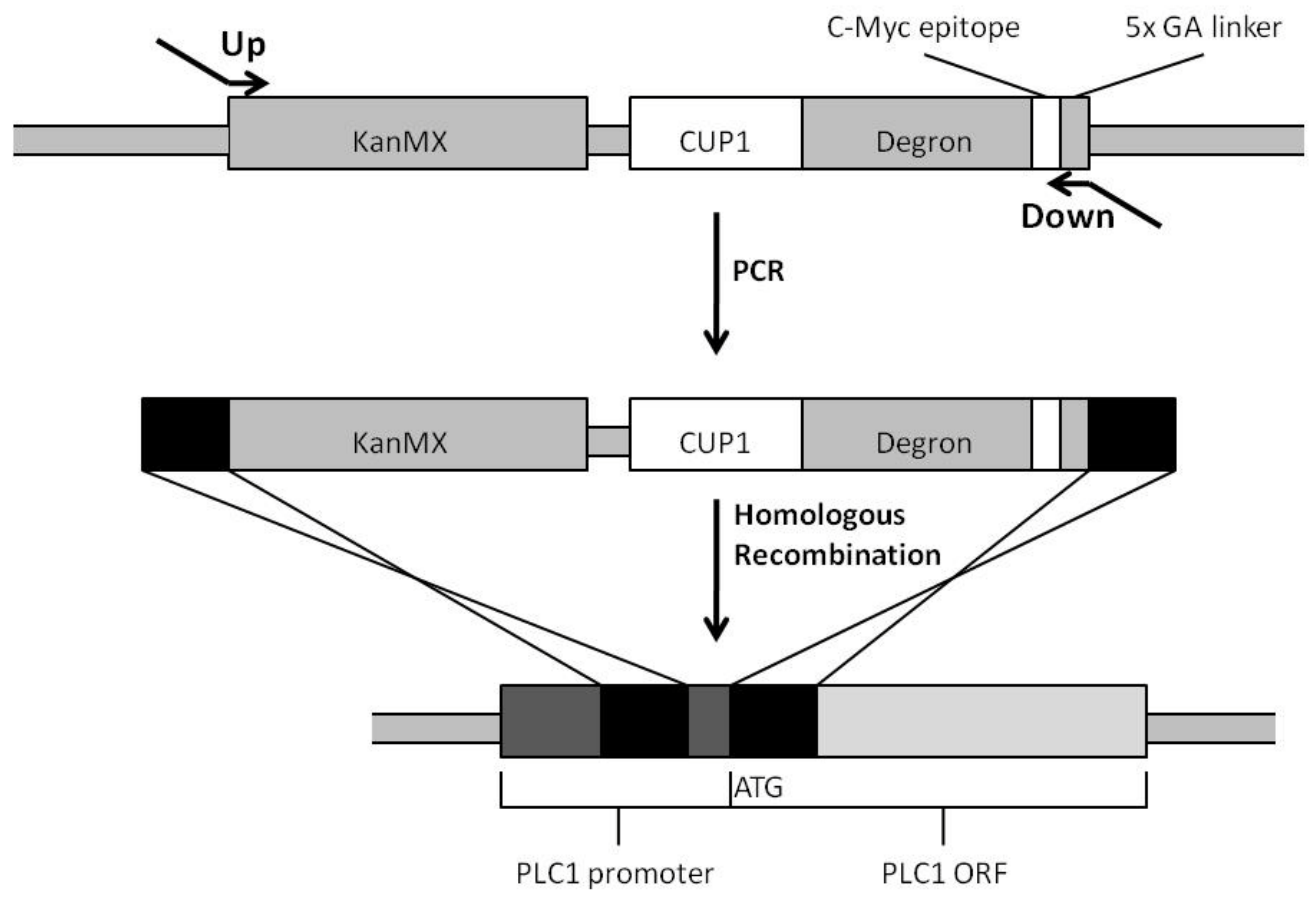

Figure 8. Insertion of a degron cassette. Integration of this construct results in partial deletion of the native promoter and fusion of the degron motif to the PLC1 loci now under control of the CUP1 promoter.

Efficient ubiquitination of the degron-tagged protein for rapid degradation requires high expression levels of Ubr1p. For this reason a copy of UBR1 under GAL1 promoter was introduced into the cells' genome. Linearization of the plasmid pKL142 (EUROSCARF) with the restriction endonuclease Pmel or Mssl produces a HIS3-GAL1-UBR1 construct already flanked by regions for insertion into the yeast genome by homologous recombination.

$1.8 \mu \mathrm{g}$ pKL142 were linearized, cleaned on a GFX column (Macherey Nagel), and eluted with $50 \mu \mathrm{l}$ distilled water. This construct was then transformed into YB332faa1 $\Delta$ faa4 $\Delta$ fat1 1 cells as described in section 3.2.2.4. and selected for complementation of histidine auxotrophy, generating strain Y41t1U. The degron cassette, synthesized as described above, was then transformed into Y41t1U cells and selected for resistance to the antibiotic G418, generating the strain DP. Insertion of the $U B R$ construct was verified by $P C R$ as described in section 3.2.2.5. with primers UbrA/UbrB (sequences in appendix 2). These primers are both internal to the construct; therefore a band is obtained upon successful integration. Insertion of the degron construct was verified with primer combinations ORFA (PIc1A)/MB (KanB), 
MC (DegC)/ORFB (PIc1B) and ORFA/ORFB (sequences in appendix 2). In this case correct integration results in bands with the first two combinations but not with the third one.

\subsubsection{Free and Bound Fatty Acid Profile Analysis}

\subsubsection{Culture and Sampling}

Every experiment (cultures grown simultaneously) consisted of maximum 8 strains. This limit was established to match the capacity of cell culture facilities and to allow for single batch processing of all samples derived from each experiment. On every occasion the reference strain (YB526) was included within the experiment.

Preculture. $20 \mathrm{ml}$ rich raffinose media (YPR), in sterile $100 \mathrm{ml}$ flasks, were inoculated with cells scraped from agar plates by means of sterile loops. The flask's mouths were sealed with aluminum foil and the suspensions incubated over night at $30^{\circ} \mathrm{C}$ with shaking at 210 rpm. The $\mathrm{OD}_{600}$ of the precultures was determined as described in section 3.2.2.1.

Culture. $30 \mathrm{ml}$ YPR medium, in sterile $100 \mathrm{ml}$ flasks, were inoculated with aliquots of the corresponding precultures to an $\mathrm{OD}_{600}$ of 0.03 . In all cases $3 \times 30 \mathrm{ml}$ cultures were started from each preculture. The flask's mouths were sealed with aluminum foil and the suspensions were incubated at $30^{\circ} \mathrm{C}$ with shaking at $210 \mathrm{rpm}$ for $136 \mathrm{~h}$.

Sampling. Except where otherwise indicated, samples were always collected after $136 \mathrm{~h}$ of incubation; in some cases samples were additionally collected after $40 \mathrm{~h}$ (see table 1 , section 4.2.).

$2 \mathrm{ml}$ aliquots from the cultures were transferred to $2 \mathrm{ml}$ microfuge tubes and centrifuged at $4000 \mathrm{rpm}$ for 3 minutes (Eppendorf 5417R). $1 \mathrm{ml}$ from the supernatants was transferred to new $2 \mathrm{ml}$ microfuge tubes and stored at $-20{ }^{\circ} \mathrm{C}$ until used for fatty acid extractions. The remaining supernatant was discarded and the pellets were resuspended in $1 \mathrm{ml}$ distilled water. The suspensions were centrifuged at $4000 \mathrm{rpm}$ for $3 \mathrm{~min}$ and the supernatants discarded. The pellets were stored at $-20^{\circ} \mathrm{C}$ until used for extraction.

Simultaneous to sample collection the $\mathrm{OD}_{600}$ of the cultures was determined as described before. 


\subsubsection{Extraction}

Extraction of free and lipid bound fatty acids was performed by the chloroform:methanol method (Folch et al. 1957).

Samples collected as described in section 3.2.3.1. (cellular pellet fraction resuspended in $1 \mathrm{ml}$ distilled water and extracellular supernatant (SN) fraction) were transferred to $10 \mathrm{ml}$ Schliff tubes. From this point on, only glass material (tubes, pipettes and syringes) was used for the extraction. To each sample $3 \mathrm{ml}$ chloroform:methanol 2:1, $25 \mu \mathrm{l} 1 \mathrm{M} \mathrm{HCl}$ and $5 \mu \mathrm{g}$ 17:0 as internal standard for free fatty acids were added. To cellular pellet samples, ca. $0.4 \mathrm{~g}$ glass beads $(425-600 \mu \mathrm{m})$ and $10 \mu \mathrm{g}$ tri-17:0 as internal standard for bound FA were also added. The samples were vortexed at high speed $(3 \mathrm{~min}$ for cell pellet fractions, under $1 \mathrm{~min}$ for SN fractions) and extracted by shaking at $4{ }^{\circ} \mathrm{C}$ ( 2 hours for cell pellet fractions, 20 min for SN fractions).

After extraction, $1 \mathrm{ml} 0.9 \%(\mathrm{w} / \mathrm{v}) \mathrm{NaCl}$ was added to the samples, the tubes were vortexed and the phases separated by centrifugation at $1000 \mathrm{rpm}$ during $4 \mathrm{~min}$ (Eppendorf 5810R centrifuge). The lower (chloroform) phases were transferred with Pasteur glass pipettes to new $10 \mathrm{ml}$ Schliff tubes and dried under a stream of nitrogen. The extracts were resuspended in $100 \mu \mathrm{l}$ chloroform:methanol 1:1. Extracts from cell pellet fractions were split into two $50 \mu$ laliquots. The first aliquot, to be used for FFA analysis, remained in the Schliff tube; the second one, for esterified FA analysis, was transferred to a $2 \mathrm{ml}$ microfuge tube.

\subsubsection{Fatty Acid Derivatization}

Methylation of free fatty acids. The SN fraction extracts and the first aliquots from the cell pellet fraction extracts (section 3.2.3.2.) were dried under a nitrogen stream. $400 \mu \mathrm{l}$ methanol and $10 \mu \mathrm{l} 0.1 \mathrm{~g} / \mathrm{ml}$ EDAC (in methanol) were added, the tubes were briefly vortexed and the solutions were incubated at room temperature $\left(21^{\circ} \mathrm{C}\right)$. After 2 hours of incubation the methylation reaction was stopped by addition of $200 \mu$ l saturated aqueous $\mathrm{NaCl}$. Until this point only glass material was used for all procedures.

To extract the methylated fatty acids, $1 \mathrm{ml}$ hexane was added, the tubes were vortexed at high speed and centrifuged during $2 \mathrm{~min}$ at $1000 \mathrm{rpm}$ (Eppendorf 5810R centrifuge). The upper (hexane) phases were transferred to $1.5 \mathrm{ml}$ microfuge tubes and dried under a 
nitrogen stream. The extracts were resuspended in $16 \mu$ lacetonitrile and the tubes were vortexed in alternating vertical and horizontal position to wash the tubes' walls.

Transmethylation of esterified fatty acids. The remaining (second) aliquots from the cell pellet fraction extracts (section 3.2.3.2.) were dried under a nitrogen stream. $333 \mu \mathrm{l}$ methanol:toluene 1:1 and $167 \mu \mathrm{l} 0.5 \mathrm{M} \mathrm{NaOCH}$ (in methanol) were added and the solutions were incubated at room temperature $\left(21{ }^{\circ} \mathrm{C}\right)$. After $20 \mathrm{~min}$ the transmethylation reaction was stopped by adding $500 \mu \mathrm{l} 1 \mathrm{M} \mathrm{NaCl}$ (aq) and $50 \mu \mathrm{l} 37 \%$ (w/w) $\mathrm{HCl}(\mathrm{aq})$.

To extract the (trans-)methylated fatty acids, $1 \mathrm{ml}$ hexane was added, the tubes were vortexed at high speed and centrifuged during $1 \mathrm{~min}$ at $5000 \mathrm{rpm}$ (Eppendorf 5415R centrifuge). The upper phases were transferred to $1.5 \mathrm{ml}$ microfuge tubes and dried under a nitrogen stream. The extracts were resuspended in $16 \mu l$ acetonitrile and the tubes were vortexed in alternating vertical and horizontal position to wash the tubes' walls.

\subsubsection{Gas Chromatography}

The methylated fatty acids, dissolved in acetonitrile, were subjected to analysis by gas chromatography (GC) under the following conditions:

\begin{tabular}{|c|c|}
\hline Chromatographer: & Agilent GC 6890 Series \\
\hline \multirow[t]{2}{*}{ Column: } & $122-2332, \mathrm{DB}-23,30 \mathrm{~m} \times 0.25 \mathrm{~mm}$. \\
\hline & Film thickness $0.25 \mu \mathrm{m}$ (J\&W Scientific, Agilent, Böblingen) \\
\hline Split: & $15: 1$ \\
\hline Injection volume: & $1 \mu l$ \\
\hline \multirow[t]{5}{*}{ Temperature program: } & $150^{\circ} \mathrm{C} \quad 1 \mathrm{~min}$ \\
\hline & $150^{\circ} \mathrm{C}-160^{\circ} \mathrm{C}, 10^{\circ} \mathrm{C} / \mathrm{min}$ \\
\hline & $160^{\circ} \mathrm{C}-200^{\circ} \mathrm{C}, 6^{\circ} \mathrm{C} / \mathrm{min}$ \\
\hline & $200^{\circ} \mathrm{C}-250^{\circ} \mathrm{C}, 25^{\circ} \mathrm{C} / \mathrm{min}$ \\
\hline & $250^{\circ} \mathrm{C}, 4^{\circ} \mathrm{C}$ \\
\hline Carrier gas: & $\mathrm{He}(1 \mathrm{ml} / \mathrm{min})$ \\
\hline Detector: & FID \\
\hline
\end{tabular}




\subsubsection{Free Fatty Acid Analysis in PLC1 Deficient Strains.}

YB526plc1 $1 \Delta$ cells present a growth defect and fail to reach an $\mathrm{OD}_{600}$ above 1 . In consequence their FA profiles could not be determined as described above for all other strains. In order to avoid the growth defect a YB332faa1 $\Delta$ faa $4 \Delta$ fat1 $\Delta$ strain expressing a recombinant PIc1p which is degraded at high temperatures was used (strain DP, section 3.2.2.7.).

Precultures of the strains DP and Y41t1U (equivalent to DP but with a wild type PLC1) were prepared by inoculation in $20 \mathrm{ml} \mathrm{YPR}+0.1 \mathrm{mM} \mathrm{CuSO}_{4}$ in $100 \mathrm{ml}$ flasks and incubation overnight at $30^{\circ} \mathrm{C}$ with shaking at $210 \mathrm{rpm}$.

$30 \mathrm{ml} \mathrm{YPR}+0.1 \mathrm{mM} \mathrm{CuSO}_{4}$ in $100 \mathrm{ml}$ flasks were inoculated with aliquots of the corresponding precultures to an $\mathrm{OD}_{600}$ of 0.05 and incubated at $28{ }^{\circ} \mathrm{C}$ for $44 \mathrm{~h}$ with shaking at $210 \mathrm{rpm}$. Under these conditions the expression of Plc1p in the DP strain is driven by the presence of $\mathrm{Cu}^{2+}$ and its degradation is avoided by the low temperature and the low or null expression of Ubr1p (see section 3.2.2.7.).

After $44 \mathrm{~h}$ the $\mathrm{OD}_{600}$ of the cultures was determined and samples were collected for $\mathrm{FA}$ analysis as described in section 3.2.3.1. $3 \mathrm{ml} 20 \%(\mathrm{w} / \mathrm{v})$ sterile filtered galactose were added and the cultures were transferred to $37{ }^{\circ} \mathrm{C}$ for further incubation. Under these conditions high expression of Ubr1p induced by galactose and partial unfolding of the degron motif by the elevated temperature lead to proteasomal degradation of Plc1p in the DP strain but not in the Y41t1U strain. The $\mathrm{OD}_{600}$ was determined and samples for FA analysis were collected after 47 and $69 \mathrm{~h}$ of culture, i.e. 3 and $25 \mathrm{~h}$ after degradation of Plc1p in the DP strain was induced. Samples were processed and analyzed as described in sections 3.2.3.2. to 3.2.3.4.

\subsubsection{Analysis of FA in Specific Lipid Classes.}

The quantification of FA esterified in specific lipid classes requires separation of the classes before FA derivatization and analysis; this was accomplished through thin layer chromatography (TLC). 
Cultures were grown as described before (section 3.2.3.1.). Samples up to $20 \mathrm{ml}$ were collected in falcon tubes and centrifuged for $3 \mathrm{~min}$ at $3000 \mathrm{rpm}$ (Eppendorf $5810 \mathrm{R}$ centrifuge), the supernatants were discarded and the pellets resuspended in $1 \mathrm{ml}$ distilled water.

The cell suspensions were transferred to Schliff tubes and extracted as described in section 3.2.3.2. with $10 \mu \mathrm{g}$ tri-17:0 as primary internal standard. Vortexing was carried out for 4 instead of 3 minutes and shaking for 3 instead of 2 hours. The extracts were resuspended in $100 \mu \mathrm{l}$ chloroform:methanol 1:1. Samples above $20 \mathrm{ml}$ require up scaling of the extraction protocol. However, the extract derived from $20 \mathrm{ml}$ of saturated culture is sufficient for at least two TLCs.

$40 \mu \mathrm{l}$ of each extract were applied on TLC plates $(20 \times 20 \mathrm{~cm}$ silica gel 60, Merck). Standards (TAG, DAG, FFA and STE for NL; PC, PE and PS for PL) were applied on additional lanes. The plates were developed in a vertical chromatography tank lined with filter paper after $1 \mathrm{~h}$ solvent equilibration. Plates for NL analysis were developed with hexane : diethyl ether : acetic acid, 80:20:1. Plates for PL analysis were developed with chloroform : methanol : acetic acid, 65:25:8.

The plates were dried and sprayed with the non-destructive fluorescent dye ammonium 8-anilino-1-naphthalenesulfonate (ANS), $0.2 \%(w / v)$ in methanol. Lipid bands were visualized under UV light and marked with a graphite pencil.

Bands were scrapped from the plates with a metal spatula and collected in Schliff tubes. $4 \mu \mathrm{g}$ tri-15:0 were added to each sample as secondary internal standard. The secondary internal standard is used for the quantification of FA recovered after the entire process; the primary internal standard is used to calculate the recovery efficiency.

$333 \mu \mathrm{l}$ methanol:toluene $1: 1$ and $167 \mu \mathrm{l} 0.5 \mathrm{M} \mathrm{NaOCH}_{3}$ (in methanol) were added for transmethylation of FA. The samples were vortexed at high speed and the solutions were incubated at room temperature $\left(21^{\circ} \mathrm{C}\right)$ for $30 \mathrm{~min}$. The transmethylation reaction was stopped by adding $500 \mu \mathrm{l} 1 \mathrm{M} \mathrm{NaCl}(\mathrm{aq})$ and $50 \mu \mathrm{l} 37 \%(\mathrm{w} / \mathrm{w}) \mathrm{HCl}(\mathrm{aq})$.

The resulting methyl esters were extracted as described in section 3.2.3.3. and analyzed by gas chromatography as described in section 3.2.3.4. 


\subsubsection{LysoPE as Source of Ethanolamine.}

Cells auxotrophic for ethanolamine ( $p s d 1 \Delta p s d 2 \Delta)$ and unable to acylate lysoPE (ale1 1 ) for PE production, are still able to utilize lysoPE as ethanolamine source by deacylation of the molecule, release of ethanolamine from the resulting glycerophosphodiester and synthesis of PE through the Kennedy pathway (Riekhof et al. 2007). In order to test for the requirement of the phospholipases B Plb1p, Plb2p, Plb3p and Nte1p and the vacuolar lipase Atg15p in the deacylation step, the ethanolamine auxotrophic strains deficient for the genes encoding these proteins $W 303 p / b 1 \Delta p / b 2 \Delta p / b 3 \Delta n t e 1 \Delta a l e 1 \Delta p s d 1 \Delta p s d 2 \Delta$ and W303plb1 1 plb2 2 plb3 3 nte $1 \Delta a l e 1 \Delta p s d 1 \Delta p s d 2 \Delta a t g 15 \Delta$ and, as controls, the ethanolamine

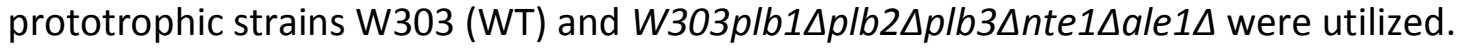

Cells were scraped from YPD-agar plates with a sterile loop and inoculated in $4 \mathrm{ml} \mathrm{YPD} \mathrm{in}$ glass tubes. The tubes were closed with a metal cap and incubated at $30{ }^{\circ} \mathrm{C}$ with shaking at $210 \mathrm{rpm}$ for $24 \mathrm{~h}$. The $\mathrm{OD}_{600}$ of the cultures was determined as described in section 3.2.2.1. A $1 \mathrm{ml}$ aliquot of the cultures was pelleted by centrifugation at $5000 \mathrm{rpm}$ for $2 \mathrm{~min}$ (Eppendorf 5417R), resuspended in $1 \mathrm{ml}$ distilled sterile water, pelleted and resuspended in water once more. Dilutions of the cell suspensions were prepared in sterile distilled water to $\mathrm{OD}_{600} 0.1,0.01$ and $0.001 .5 \mu$ aliquots of the dilutions were plated on complete SD (glucose) $+1 \%(\mathrm{v} / \mathrm{v})$ Nonident P40 agar plates containing either no ethanolamine at all, $5 \mathrm{mM}$ ethanolamine or $0.4 \mathrm{mM}$ lysoPE. The plates were allowed to dry, closed and incubated at $30{ }^{\circ} \mathrm{C}$ for 10 days.

\subsubsection{Cell number and cell viability}

In order to verify the correlation between $\mathrm{OD}_{600}$ and the number of cells in a culture, cell counting was performed.

Cultures were grown as described in section 3.2.3.1. After $136 \mathrm{~h}$ incubation the $\mathrm{OD}_{600}$ of the cultures were determined as described in 3.2.2.1. and aliquots were diluted with sterile distilled water by a factor of 20 . An aliquot of the dilution was loaded on a hemocytometer and cells were counted under a light microscope (Olympus BX51). 
In order to assess the effect of gene deletions on cell fitness, a viability test was carried out. The 20x dilutions used for cell counting were further diluted by a factor of 800 with sterile distilled water (dilution from culture by a factor of 16000). $100 \mu$ l aliquots of these dilutions were plated on YPD agar plates, allowed to dry and incubated at $30{ }^{\circ} \mathrm{C}$ for $3-4$ days. The number of colonies appearing on the plates was counted and used to calculate the fraction of viable cells in the culture. 



\section{Results}

\subsection{S. cerevisiae mutants}

More than 150 mutant lines were produced during this work. The establishment of this large collection constitutes of course an important result within this project but the actual list of mutants and description of their genotypes is omitted from the main text and presented in appendix 1 . The results demonstrating the genotypes of the mutant lines (PCR products resolved on agarose gels) are not presented.

\subsection{Optical density and cell growth}

Figure 9 presents a standard growth curve for S. cerevisiae cells of YB332 background in YPR media. There is a lag phase from 0 to $18 \mathrm{~h}$ (in some cases this extends up to $24 \mathrm{~h}$ ) followed by an exponential growth phase up to $40 \mathrm{~h}$. After 40 hours the cells undergo a diauxic shift during which growth is usually arrested for 2 to 4 hours and then resumed at a lower rate than during exponential phase for approximately 20 hours. During this period the $\mathrm{OD}_{600}$ reaches its maximum and then declines slightly. Within 65 and $70 \mathrm{~h}$ the cells enter stationary phase. Notice that the optical density at the end of exponential growth phase is very similar to the $\mathrm{OD}_{600}$ at late stationary phase. 


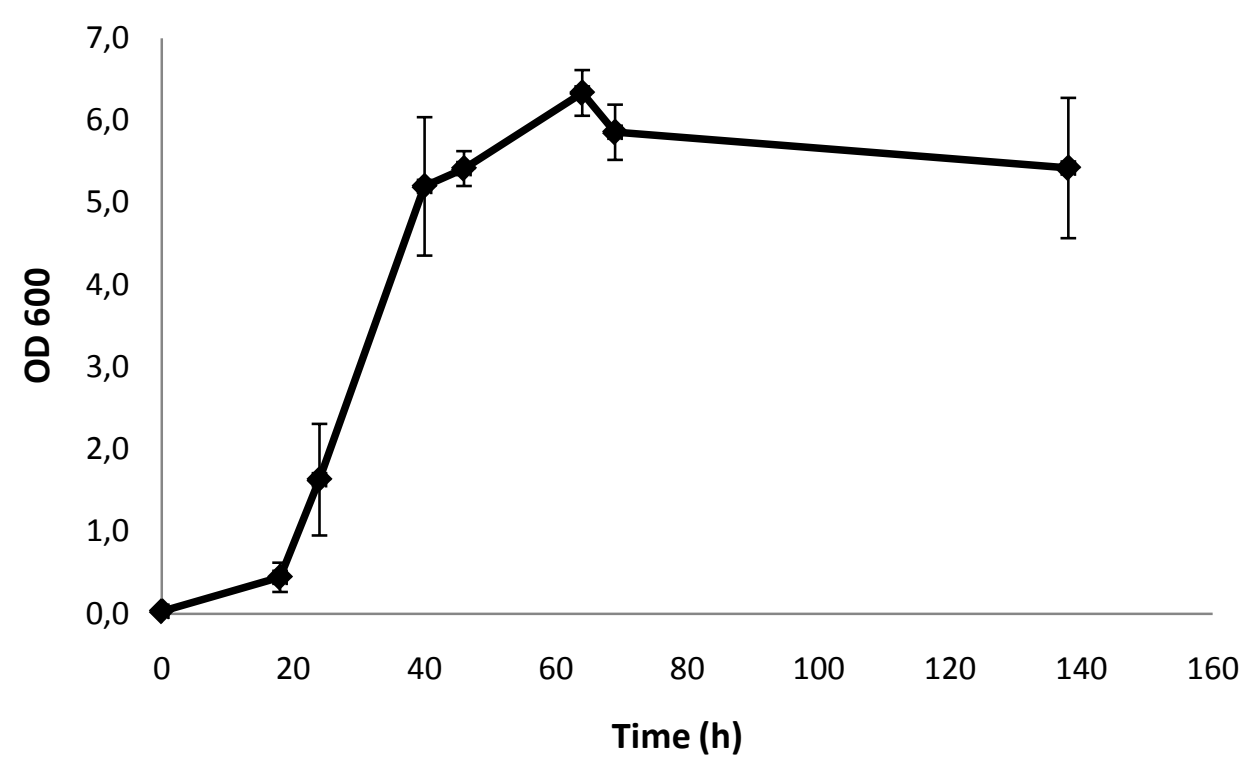

Figure 9. Growth curve YB526 in YPR. The plotted values correspond to the average of two independent cultures with three replicas each.

Table 1 presents the $\mathrm{OD}_{600}$ at $136 \mathrm{~h}$ of all mutants whose FA profiles are presented in the next section (4.3.) as well as of other mutants for which no FA profiles will be presented.

In some cases the $\mathrm{OD}_{600}$ was also determined after 40 hours of culture. This results in a very similar value to $\mathrm{OD}_{600}$ at $136 \mathrm{~h}$ for the same culture, indicating that the exponential growth phase has approximately the same duration for cultures of different strains and therefore that they grow at a similar relative rate. 
Table 1. $O D_{600}$ of various S. cerevisiae strains. Cells were grown in $30 \mathrm{ml} \mathrm{YPR} \mathrm{media} \mathrm{at} 30^{\circ} \mathrm{C}$. YB332: wild

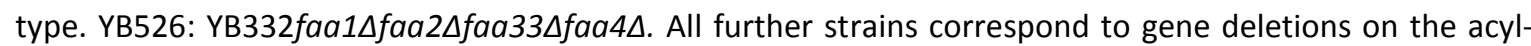
CoA synthetase (ACS) deficient strain YB526 and are labeled only by these additionally deleted genes.

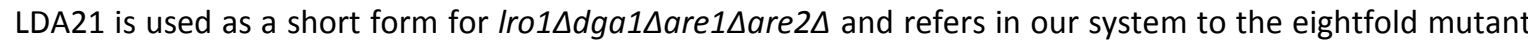

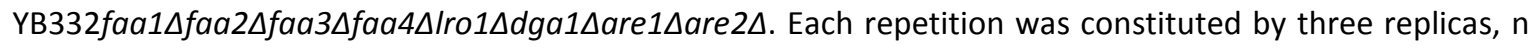
corresponds to the total number of replicas.

\begin{tabular}{|c|c|c|c|c|c|c|}
\hline \multirow[b]{2}{*}{ Strain } & \multicolumn{3}{|c|}{$136 \mathrm{~h}$} & \multicolumn{2}{|c|}{$40 \mathrm{~h}$} & \multirow[b]{2}{*}{$\mathrm{n}$} \\
\hline & $\mathrm{OD}_{600}$ & St.dev. & $\mathrm{n}$ & $\mathrm{OD}_{600}$ & St. dev & \\
\hline YB332 & 7.2 & 0.2 & 3 & 6.8 & 0.1 & 3 \\
\hline YB526 & 6.3 & 0.6 & 72 & 6.1 & 0.4 & 48 \\
\hline plb1 $\Delta$ & 5.8 & 0.1 & 3 & 5.6 & 0.2 & 3 \\
\hline $\mathrm{plb} 2 \Delta$ & 6.0 & 0.1 & 3 & 5.9 & 0.1 & 3 \\
\hline plb3 $\Delta$ & 5.9 & 0.3 & 3 & 6.0 & 0.3 & 3 \\
\hline nte1 $\Delta$ & 5.6 & 0.2 & 3 & 5.3 & 0.1 & 3 \\
\hline plb1 $\Delta$ plb2 $\Delta$ plb3 $\Delta$ & 6.2 & 0.5 & 3 & & & \\
\hline $\operatorname{tg} \mid 2 \Delta$ & 6.8 & 0.2 & 3 & & & \\
\hline $\operatorname{tg} \mid 3 \Delta$ & 7.1 & 0.2 & 6 & 6.4 & 0.3 & 3 \\
\hline $\operatorname{tg} \mid 4 \Delta$ & 6.5 & 0.2 & 6 & 6.1 & 0.1 & 3 \\
\hline $\operatorname{tg} \mid 5 \Delta$ & 5.8 & 0.2 & 6 & 5.5 & 0.2 & 3 \\
\hline $\operatorname{tg}|3 \Delta \operatorname{tg}| 4 \Delta \operatorname{tg} \mid 5 \Delta$ & 6.8 & 0.3 & 3 & & & \\
\hline Iro1 $\Delta$ & 7.4 & 0.4 & 6 & & & \\
\hline$|\operatorname{ro} 1 \Delta \operatorname{tg}| 3 \Delta$ & 6.5 & 0.2 & 3 & 5.9 & 0.1 & 3 \\
\hline $\operatorname{dga} 1 \Delta$ & 6.3 & 0.2 & 6 & 6.1 & 0.1 & 3 \\
\hline $\operatorname{tg} \mid 3 \Delta$ dga1 $\Delta$ & 6.8 & 0.1 & 3 & 6.1 & 0.2 & 3 \\
\hline Iro1 $\Delta$ dga1 $\Delta$ & 6.2 & 0.1 & 3 & & & \\
\hline Iro1 $\Delta$ nte1 $\Delta$ & 6.2 & 0.2 & 6 & 6.0 & 0.1 & 6 \\
\hline nte $1 \Delta$ dga $1 \Delta$ & 6.7 & 0.1 & 3 & 5.9 & 0.1 & 3 \\
\hline Iro $1 \Delta$ nte $1 \Delta$ dga $1 \Delta$ & 6.1 & 0.2 & 3 & 6.0 & 0.2 & 3 \\
\hline nte $1 \Delta \operatorname{tg} \mid 3 \Delta$ & 6.2 & 0.1 & 3 & 6.2 & 0.1 & 3 \\
\hline $\operatorname{are} 1 \Delta \operatorname{are} 2 \Delta$ & 7.5 & 0.2 & 3 & & & \\
\hline $\operatorname{tg} \mid 1 \Delta$ & 6.7 & 0.3 & 3 & & & \\
\hline yeh1 $1 \Delta$ & 6.6 & 0.3 & 3 & & & \\
\hline yeh2A & 6.8 & 0.1 & 3 & & & \\
\hline yeh $1 \Delta$ yeh $2 \Delta \operatorname{tg} \mid 1 \Delta$ & 6.9 & 0.6 & 6 & & & \\
\hline yeh1 $\Delta$ yeh $2 \Delta \operatorname{tg}|1 \Delta \operatorname{tg}| 3 \Delta$ & 7.2 & 0.6 & 6 & & & \\
\hline LDA21 & 5.8 & 0.4 & 12 & 6.2 & 0.5 & 6 \\
\hline LDA21nte1 $\Delta$ & 5.7 & 0.3 & 6 & 5.8 & 0.2 & 3 \\
\hline $\operatorname{LDA} 21 \operatorname{tg} \mid 3 \Delta$ & 5.6 & 0.5 & 6 & 5.9 & 0.1 & 3 \\
\hline spo14 $\Delta$ & 5.3 & 0.2 & 3 & 5.2 & 0.3 & 3 \\
\hline $\operatorname{taz} 1 \Delta$ & 5.5 & 0.1 & 3 & 5.7 & 0.1 & 3 \\
\hline ale1 $\Delta$ & 5.4 & 0.1 & 3 & & & \\
\hline nte1 $\Delta$ ale1 $\Delta$ & 5.5 & 0.3 & 3 & & & \\
\hline nte $1 \Delta$ spo $14 \Delta$ & 5.0 & 0.1 & 3 & 5.3 & 0.0 & 3 \\
\hline
\end{tabular}


Table 1. Continued from previous page.

\begin{tabular}{|c|c|c|c|c|c|c|}
\hline \multirow[b]{2}{*}{ Strain } & \multicolumn{3}{|c|}{$136 \mathrm{~h}$} & \multicolumn{3}{|c|}{$40 \mathrm{~h}$} \\
\hline & $\mathrm{OD}_{600}$ & St.dev. & $\mathrm{n}$ & $\mathrm{OD}_{600}$ & St. dev & $n$ \\
\hline Iro1 $\Delta$ spo14 & 5.8 & 0.3 & 3 & & & \\
\hline $\operatorname{atg} 1 \Delta$ & 6.6 & 0.1 & 6 & 6.3 & 0.2 & 3 \\
\hline $\operatorname{tg} \mid 3 \Delta \operatorname{atg} 15 \Delta$ & 6.5 & 0.1 & 6 & 6.2 & 0.1 & 6 \\
\hline $\operatorname{tg} \mid 4 \Delta \operatorname{atg} 15 \Delta$ & 7.2 & 0.3 & 3 & & & \\
\hline $\operatorname{tg}|3 \Delta \operatorname{tg}| 4 \Delta \operatorname{tg} \mid 5 \Delta \operatorname{atg} 15 \Delta$ & 7.4 & 0.1 & 3 & & & \\
\hline $\operatorname{tg} \mid 3 \Delta \operatorname{atg} 1 \Delta$ & 6.9 & 0.2 & 6 & & & \\
\hline $\operatorname{tg}|3 \Delta \operatorname{tg}| 4 \Delta \operatorname{tg} \mid 5 \Delta \operatorname{atg} 1 \Delta$ & 6.8 & 0.2 & 3 & & & \\
\hline Iro1 $\Delta \operatorname{atg} 15 \Delta$ & 6.9 & 0.3 & 3 & 5.8 & 0.1 & 3 \\
\hline LDA21atg15 $\Delta$ & 6.7 & 0.2 & 6 & 6.2 & 0.1 & 3 \\
\hline LDA21atg1 $\Delta$ & 5.7 & 0.2 & 6 & & & \\
\hline nte $1 \Delta \operatorname{atg} 15 \Delta$ & 6.7 & 0.5 & 6 & 6.0 & 0.1 & 3 \\
\hline Iro1 $\Delta$ atg $15 \Delta$ nte $1 \Delta$ & 6.6 & 0.5 & 6 & 6.1 & 0.3 & 3 \\
\hline nte1 $\Delta \operatorname{tg} \mid 3 \Delta \operatorname{atg} 15 \Delta$ & 6.5 & 0.2 & 3 & 5.8 & 1.0 & 3 \\
\hline Iro1 $\Delta$ nte1 $\Delta \operatorname{tg} \mid 3 \Delta$ & 6.9 & 0.4 & 6 & 6.3 & 0.4 & 6 \\
\hline Iro1 $\Delta \operatorname{atg} 15 \Delta \operatorname{tg} \mid 3 \Delta$ & 6.9 & 0.2 & 3 & 5.9 & 0.1 & 3 \\
\hline nte $1 \Delta \operatorname{tg}|3 \Delta \operatorname{atg} 15 \Delta|$ ro $1 \Delta$ & 7.1 & 0.1 & 3 & 6.0 & 0.1 & 3 \\
\hline $\operatorname{atg} 22 \Delta$ & 6.1 & 0.4 & 6 & 6.6 & 0.1 & 3 \\
\hline pep4 $\Delta$ & 7.0 & 0.3 & 6 & 6.9 & 0.1 & 3 \\
\hline pep $4 \Delta \operatorname{atg} 22 \Delta$ & 7.6 & 0.2 & 3 & & & \\
\hline pep4 $\Delta \operatorname{atg} 15 \Delta$ & 7.5 & 0.2 & 3 & & & \\
\hline $\operatorname{atg} 1 \Delta \operatorname{pep} 4 \Delta$ & 6.8 & 0.1 & 3 & & & \\
\hline $\operatorname{atg} 1 \Delta \operatorname{atg} 22 \Delta$ & 6.2 & 0.3 & 6 & & & \\
\hline $\operatorname{atg} 15 \Delta \operatorname{atg} 22 \Delta$ & 7.3 & 0.2 & 3 & & & \\
\hline $\operatorname{atg} 1 \Delta \operatorname{atg} 15 \Delta$ & 6.7 & 0.2 & 3 & & & \\
\hline $\operatorname{lp} x 1 \Delta$ & 7.2 & 0.2 & 9 & & & \\
\hline $\operatorname{tg} \mid 3 \Delta \operatorname{lpx} 1 \Delta$ & 7.4 & 0.3 & 3 & & & \\
\hline LDA21/px1 & 6.5 & 0.2 & 3 & & & \\
\hline $\operatorname{atg} 1 \Delta \operatorname{lpx} 1 \Delta$ & 6.9 & 0.2 & 3 & & & \\
\hline $\operatorname{atg} 15 \Delta \mid \mathrm{px} 1 \Delta$ & 7.2 & 0.2 & 3 & & & \\
\hline $\operatorname{atg} 15 \Delta \operatorname{lp} \times 1 \Delta \operatorname{tg} \mid 3 \Delta$ & 7.8 & 0.3 & 6 & & & \\
\hline $\operatorname{pex3} \Delta$ & 6.4 & 0.1 & 3 & & & \\
\hline $\operatorname{tg} \mid 3 \Delta \operatorname{pex} 3 \Delta$ & 7.0 & 0.3 & 3 & & & \\
\hline $\operatorname{atg} 1 \Delta \operatorname{pex} 3 \Delta$ & 6.5 & 0.1 & 3 & & & \\
\hline $\operatorname{atg} 15 \Delta \operatorname{pex} 3 \Delta$ & 7.3 & 0.1 & 3 & & & \\
\hline LDA21pex3A & 6.4 & 0.2 & 3 & & & \\
\hline LDA21atg $1 \Delta$ pex3 $\Delta$ & 7.1 & 0.3 & 6 & & & \\
\hline
\end{tabular}


At $136 \mathrm{~h}$ the $\mathrm{OD}_{600}$ ranges from a minimum of 5 in the YB526nte1 $\Delta$ spo14 $\Delta$ mutant to a

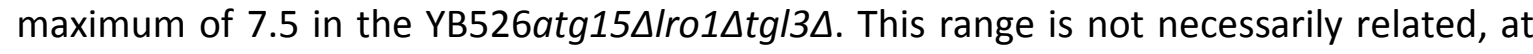
least not exclusively, to the genetic constitution of the mutants: The reference strain, YB526, was cultured 24 times (3 replicas each time) and results in an average ${ } D_{600}$ of 6.3 with a standard deviation of 0.6 . The $\mathrm{OD}_{600}$ values obtained for the YB526 throughout this 24 repetitions range from 5 to 7.3. Similarly, at $40 \mathrm{~h}$ the $O D_{600}$ for the various strains

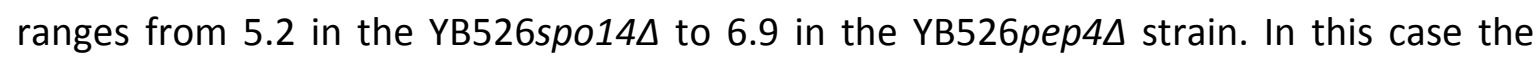
$\mathrm{OD}_{600}$ of the YB526 strain was determined in 16 repetitions (3 replicas each time) resulting in an average of 6.1 , a standard deviation of 0.4 , a minimum of 5.3 and a maximum of 7.1. These variations in growth represent a problem for our quantification of FA content (next section) since aliquots of different cultures will contain a different number of cells. To overcome this, the FA concentrations in the following section are expressed per $\mathrm{OD}_{600}$ unit.

The relation between $\mathrm{OD}_{600}$ and cell number was verified using a subset of the strains utilized for FFA and lipid quantification (figure 10). Although the number of cells per $\mathrm{OD}_{600}$ unit is not exactly constant, no large variations of this quantity where encountered within strains of YB526 background. Wild type (YB332) cells, however, show a significantly higher number of cells per OD unit. This difference cannot be simply ascribed to the presence or absence of intracellular FFA since the YB332atg15 (no FFA) has less cells per $\mathrm{OD}_{600}$ unit than the YB332. 


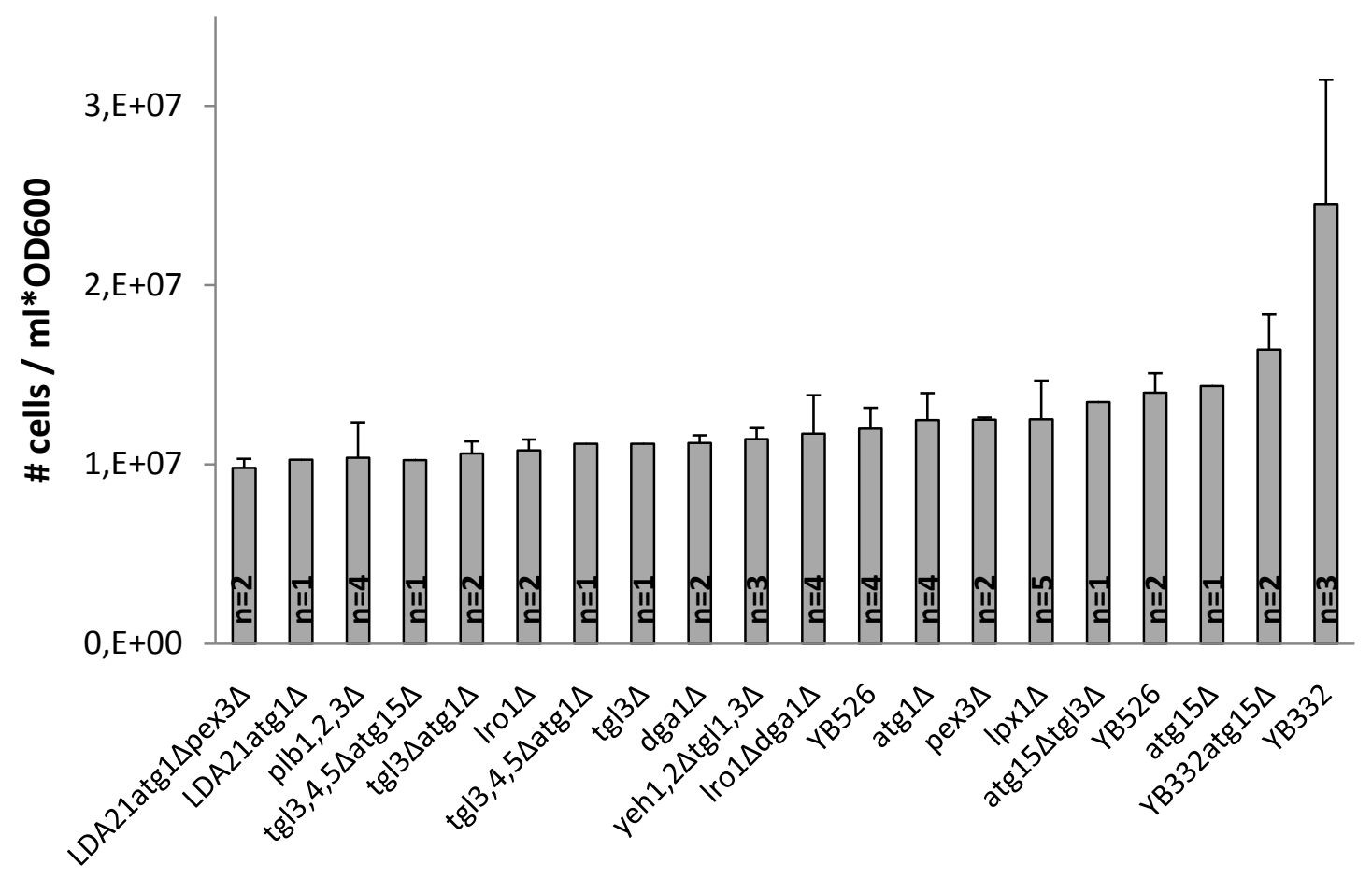

Figure 10. Number of cells per $\mathrm{ml}$ per $\mathrm{OD}_{600}$ unit at late stationary phase (136 h).

Except where YB332 is indicated, all genes referred in the figure label correspond to deletions on the YB526

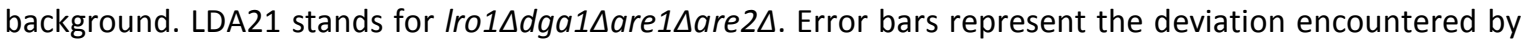
counting the number of cells in various aliquots of a single culture. Columns without error bars correspond to cultures where cell counting was carried out only once. For the YB526 strain cell count was performed for two independent cultures.

\subsection{Quantification of free and esterified fatty acids}

\subsubsection{Multiple candidate pathways for PL deacylation: PLBs, NL metabolism, PLD, LPLATs, autophagy and the peroxisome.}

Figures $11 \mathrm{~A}$ and $\mathrm{B}$ present the content and profile of free and esterified FA in wild type

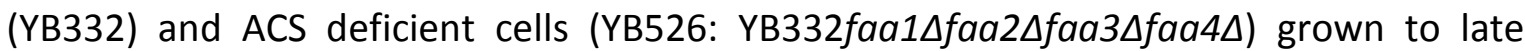
stationary phase (136 hours) in YPR media. Figures thereafter (12 to 24 ) omit the actual profile and present both the total content of free and of esterified fatty acids (i.e. the addition of all major fatty acid classes $14: 0$ to 18:1) in YB526 cells and cells of YB526 background carrying additional gene deletions. These global quantities "total fatty acids" 
correlate directly to lipid deacylation and lipid accumulation and, in comparison to the actual individual FA profiles, simplify the representation of data making it more accessible to analysis. In some cases, however, fatty acid profiles contain relevant traits that would be lost within the global quantity and are therefore presented in table 2 and appendix 3. For simplicity, data series in these figures and tables are labeled only by the genes additionally deleted on the YB526 background. For example the data point labeled

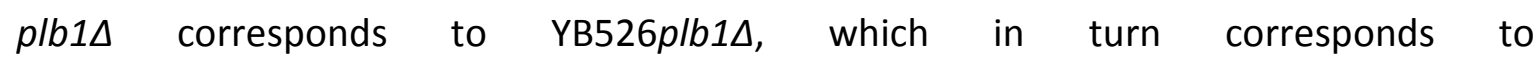
YB332faa1 $\Delta$ faa $\Delta$ faa3 $\Delta$ faa4 $\Delta$ plb1 $\Delta$. In addition to the abbreviated name YB526 for YB332faa1 $\Delta$ faa2 $\Delta$ faa3 $\Delta$ faa4 4 , the abbreviated name LDA21 is used for YB526/ro1 $\Delta$ dga1 $\Delta$ are $\Delta 1$ are2 $\Delta$.

The sampling procedure (section 3.2.3.1.) allows us to distinguish FFA secreted into the culture medium from those retained in (or imported into) the cell. Distinction between intra- and extracellular FFA has in the past provided very useful information regarding the mechanisms of FA transport across the plasma membrane and the correlation of that process or the process of FFA formation with the metabolic status of the cell (Mora 2006; Scharnewski 2005; Scharnewski et al. 2008). Such information, however, comes through an analysis of FA export and import as a dynamic process, i.e. from the evolution of the intra- and extracellular FFA pools relative to each other rather than from the absolute or relative amounts at a given moment. Under the single time point approach behind the present set of results, the choice of which will be commented on ahead, that distinction provides no further insight into the mechanisms of lipid deacylation and FFA formation and is therefore omitted. The values presented in the figures and tables as total free fatty acids correspond to the addition of intra- and extracellular FFA for a given sample.

The transmethylation method utilized for quantification of esterified fatty acids (section 3.2.3.3.), does not distinguish between different acyl esters; consequently the values reported as esterified (or bound) FA correspond to FA in all lipid classes. Determination of FA in specific lipid classes requires separation of the lipid classes prior to quantification; results from experiments where we proceeded in such way are reported in section 4.4 .

As mentioned above, all measurements reported in this section correspond to samples from late stationary phase. At the very origin of this work we expected the FFA pool that 
accumulates in ACS deficient cells to be almost entirely derived directly from phospholipid deacylation. Furthermore, despite being aware that several diverse cellular processes involve lipid deacylation, we expected the contributions of these numerous processes to be relatively small, while the vast majority of FFA would have been derived from PL deacylation through a single central mechanism mediated by only a few enzymes (namely phospholipases B). Under such hypothesis, and given that the dynamic behavior of the FFA pool presents correlations with progression through the various culture stages (Mora 2006; Scharnewski 2005; Scharnewski et al. 2008), it was reasonable to attempt not only a verification of candidate proteins involvement in FFA production, but to simultaneously begin an evaluation of their contribution throughout the entire culture span. Consequently our initial measurements always included samples throughout exponential and stationary phase. However, as our initial candidates turned out not to be involved in the deacylation phenomenon observed (figure 12), the number of genes to be considered, and therefore the number of strains to be tested, kept growing, leading us to reduce the number of samples per strain to only late stationary phase (136 h) while postponing the study of the phenomenon's time dependence to a later stage of the research. Genes the deletion of which does affect the FFA content where eventually identified (see for example figures 13, 17 and 20). However, the complexity of the pathways involved, as well as the fact that none of these pathways accounts on its own for a complete majority of FFA production, demanded an ever growing number of mutant strains representing diverse combinations of gene deletions to be tested. It was therefore necessary to make a choice between either deepening the study of the metabolic transit of fatty acids as a dynamic phenomenon at the deacylation points initially identified, or attempting to create a more extensive, though static, picture of the lipid deacylation network. The second option was taken since a more comprehensive view of the network should enormously facilitate the choice and design of experiments to probe the metabolic flow of FA. Late stationary phase samples are ideally suited for this purpose since, regardless of how the behavior of a particular protein evolves throughout the culture, its overall effect on lipid deacylation is reflected on this final FFA content. 
The FA concentrations are expressed per $\mathrm{OD}_{600}$ unit, making it approximately proportional to the amount of FA per cell (the validity of this approximation was addressed through the results presented in figure 10) and therefore compensating for variations in the growth of different cultures. This of course compensates only for variations in the final cell number or saturation density, and not for possible divergences in growth speed (and therefore in cell number during exponential phase). This, however, should not introduce too large an error since, with exception of strains carrying a deletion of PLC1 which will be addressed separately, the different strains grow not only to a similar saturation density but also at very similar rates (compare $\mathrm{OD}_{600}$ at $40 \mathrm{~h}$ with $\mathrm{OD}_{600}$ at $136 \mathrm{~h}$ for individual strains, table 1 , the similarity in the values indicates that exponential phase has approximately the same duration in all strains).

Each experiment (measurements carried out on cultures grown simultaneously) was constituted by a maximum of eight strains, with three replicas derived from a single preculture per strain. Some strains, however, where cultured more than once. In particular the YB526 strain, which constitutes the genetic background on which all other strains where constructed, was grown once in every experiment. At this stage the results of the YB526 strain constitute the reference against which the FA content of all other strains is compared, during data collection, however, it was additionally used as an experimental control: If in an experiment the results of the YB526 strain deviated largely from its normal behavior, the results of the accompanying strains needed to be discarded, or at least handled with extreme care, since any variations found in their FA content could be derived from experimental conditions rather than from their genetic constitution. Some elements of the experimental procedure, such as lipid extraction, FA derivatization and gas chromatography, are extremely robust. Other elements, however, are susceptible to experimental error. In particular, cell culture and FFA production throughout it might be affected by uncontrollable and often unnoticeable minor changes in temperature or in the continuity of the cultures agitation derived from the intense use of the cell culture facilities.

The values reported in the figures and tables correspond to the average (arithmetic mean) of all replicas and all repetitions taken together for each particular strain, error bars correspond to standard deviation. 
A

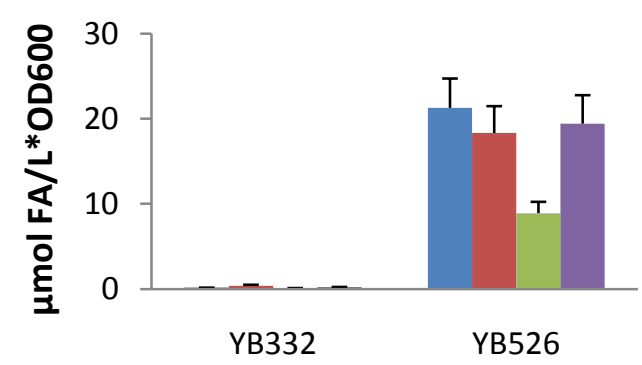

B

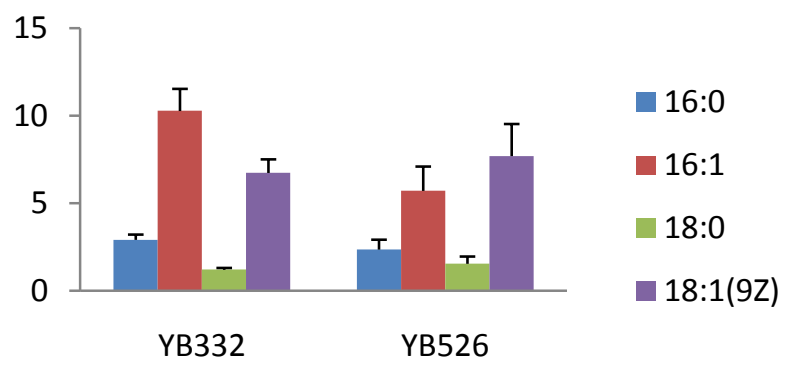

Figure 11. Free (A) and bound (B) FA in wild type (YB332) and acyl-CoA synthetase deficient (YB526) yeast cells. Deletion of the genes coding for acyl-CoA synthetases leads to the accumulation of large amounts of endogenously produced free fatty acids. YB332, $n=3 ; Y B 526, n=72$.

\section{Phospholipases B}

PLBs were initially considered as the most likely candidates to mediate the production of the FFA pool that accumulates in YB526 cells. These were therefore the first genes to be deleted. Their deletion, however, has no effect on the size of the FFA pool (figure 12).
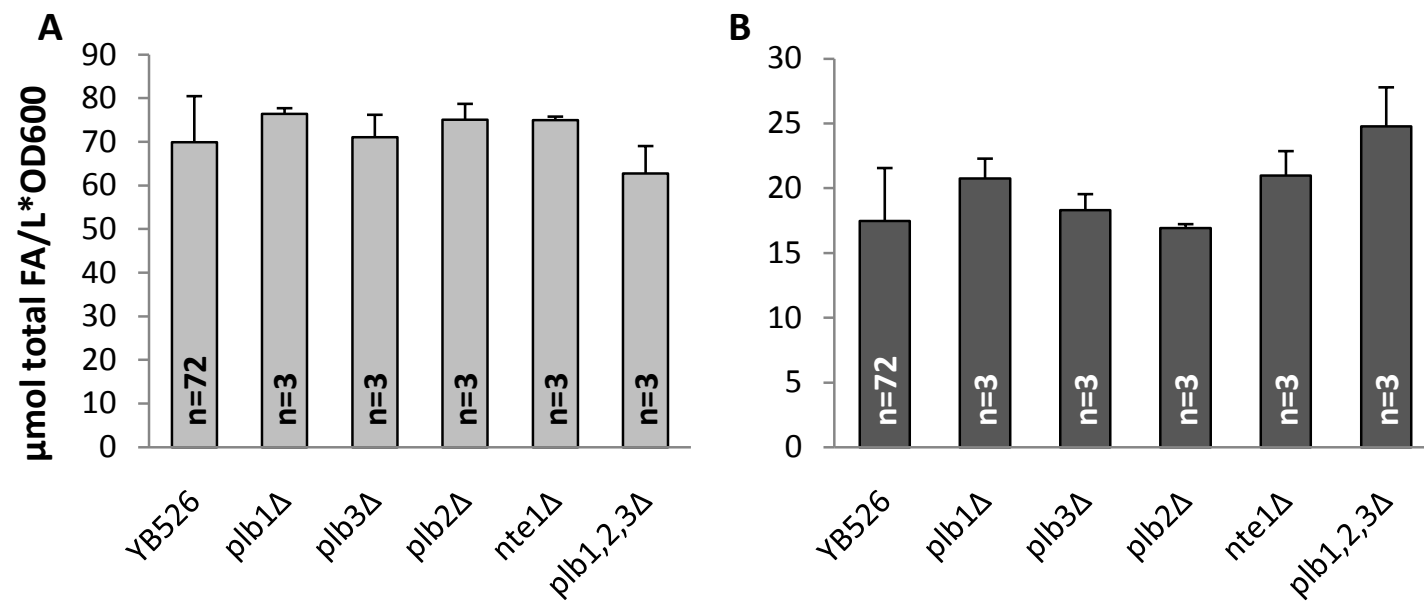

Figure 12. Free (A) and bound (B) FA in PLB deficient YB526 cells. Deletion of the genes coding for phospholipases B has no effect on the size of the FFA pool. 


\section{Neutral lipid metabolism}

Having out ruled PLBs as the mediators of FFA production under our culture conditions, TAG mobilization was considered as a possible source for the FFA pool. Furthermore, TAG synthesis by the PDAT Lro1p can mediate FA transit form the PL to the FFA pool through the TAG pool. Deletion of TGL3 or LRO1 on the YB526 strain does in fact lead to a reduced amount of FFA. The reduction in FFA content is, in both cases, accompanied by an increased accumulation of esterified FA (figure 13).
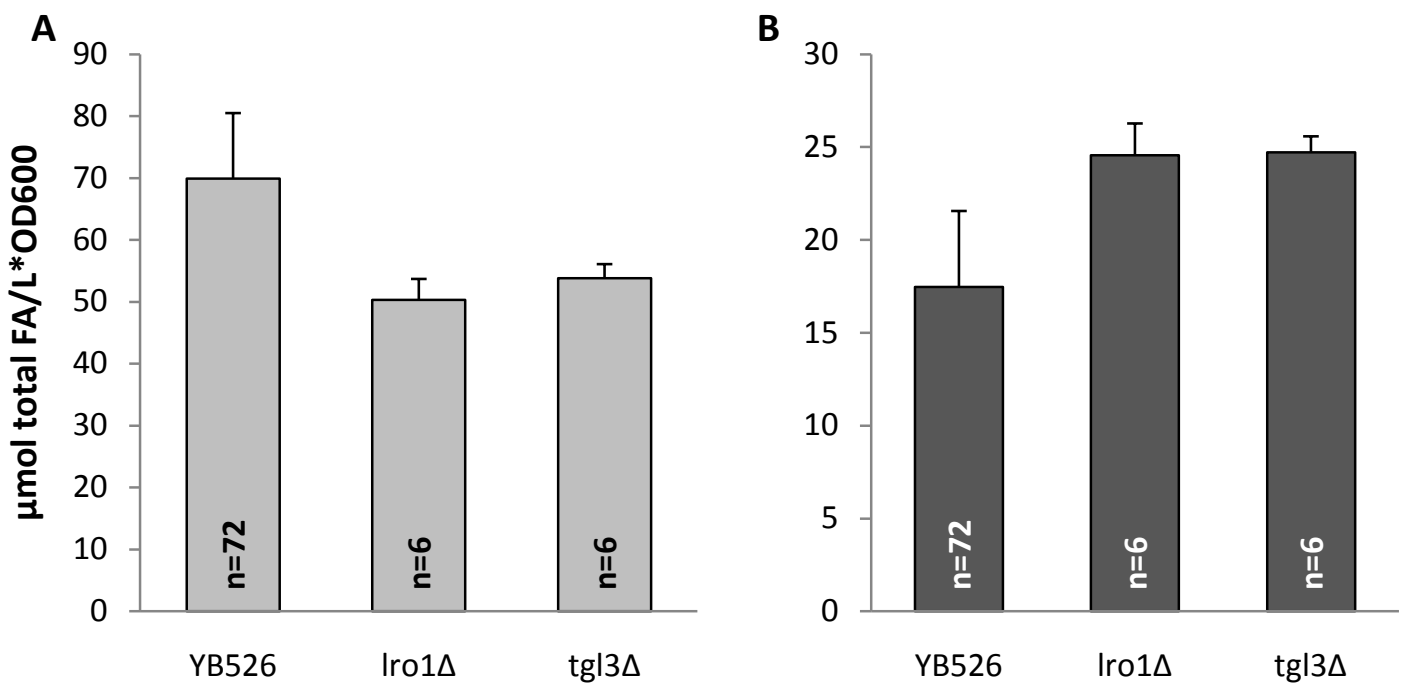

Figure 13. Deletions of the PDAT LRO1 or the TAG lipase TGL3 on YB526 cells. Either of these deletions reduces the FFA content (A) and causes an increased accumulation of bound FA (B). 
In addition to $T G L 3$, three other genes code for proteins with TAG lipase activity in yeast. Under our experimental conditions, however, their deletion has no effect on the size of the FFA pool and does not lead to an increase in esterified FA content (figure 14). In fact, deletion of TGL2 causes a decrease in esterified FA content.

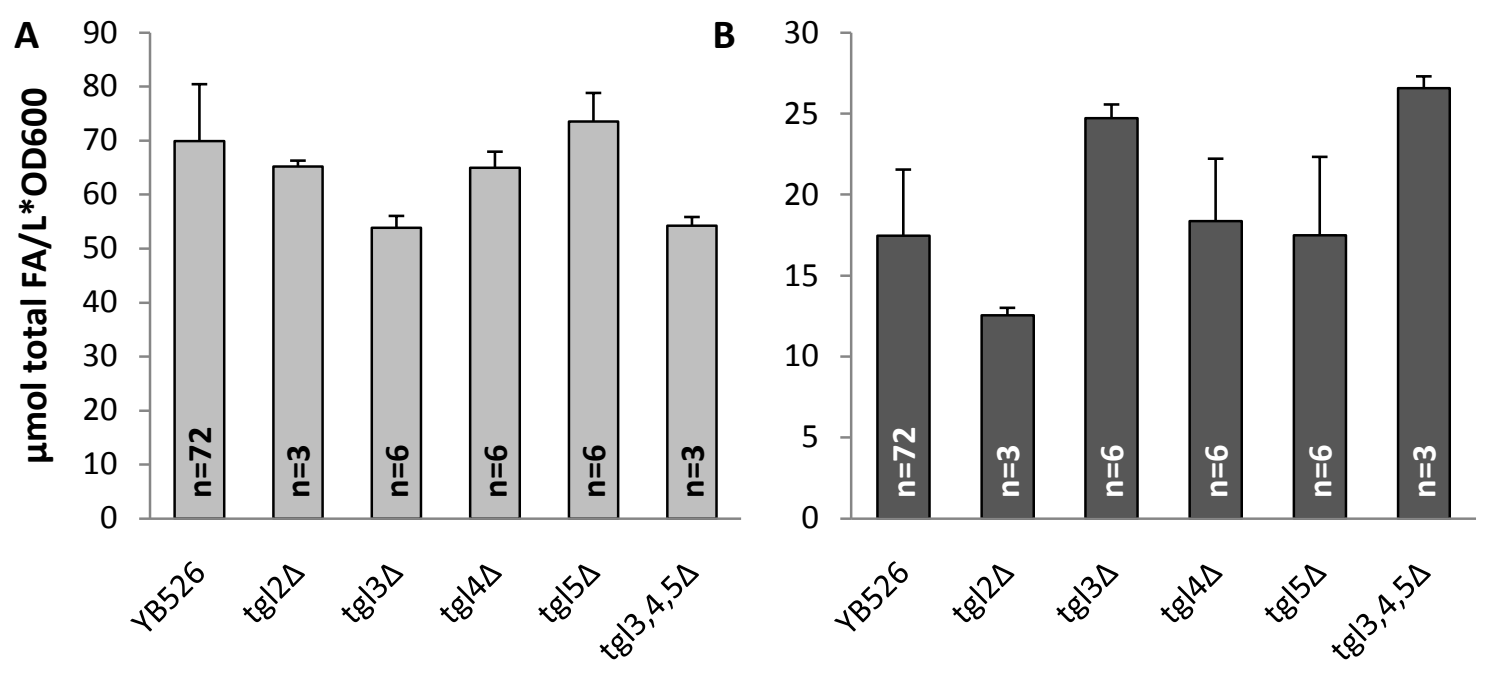

Figure 14. Single and multiple deletions of the TAG lipases TGL2, TGL3, TGL4 and TGL5 on YB526 cells. Deletion of TAG lipases other than TGL3 has no effect on the size of the FFA pool (A) and does not lead to an increased accumulation of esterified FA (B).

In addition to the PDAT Lro1p, the DGAT Dga1p is largely responsible for TAG synthesis in yeast. Since the deletion of TGL3 indicated that TAG mobilization is a major source for the FFA pool of YB526 cells, and furthermore given that partially impaired TAG synthesis in the YB526/ro1 $\Delta$ strain is accompanied by a reduced FFA pool, a similar effect could be expected from the deletion of DGA1. This, however, is not the case. The YB526dga1 strain contains as much FFA as the YB526, its esterified FA pool, in contrast, is reduced (figure 15). This reveals that the involvement of TAG mobilization in FFA formation, while mechanistically simple, is metabolically complex. In order to gain further insight into this matter, strains of YB526 background carrying combined deficiencies of proteins involved in TAG metabolism were produced (figure 15). The effects of $L R O 1$ and TGL3 deletion on the FFA pool are not additive, as the YB526/ro1 $\Delta \operatorname{tg} / 3 \Delta$ strain has the same FFA content as the strains with only one of these genes deleted. Most outstanding, the strains

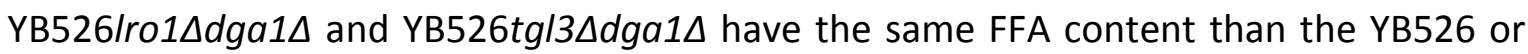


YB526dga1 $\Delta$ strains. This indicates that the effect of $L R O 1$ or $T G L 3$ deletion on the FFA pool is either eliminated or counterbalanced by the absence of DGA1. The esterified FA

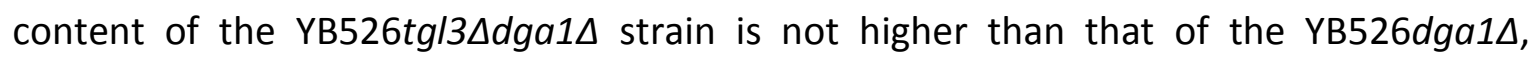
indicating that the increase in esterified FA content caused by deletion of TGL3 on the YB526 background is no longer present in the DGA1 deficient background. In contrast, the YB526/ro1 $\Delta d g a 1 \Delta$ strain contains more esterified FA than the YB526dga1 $\Delta$.
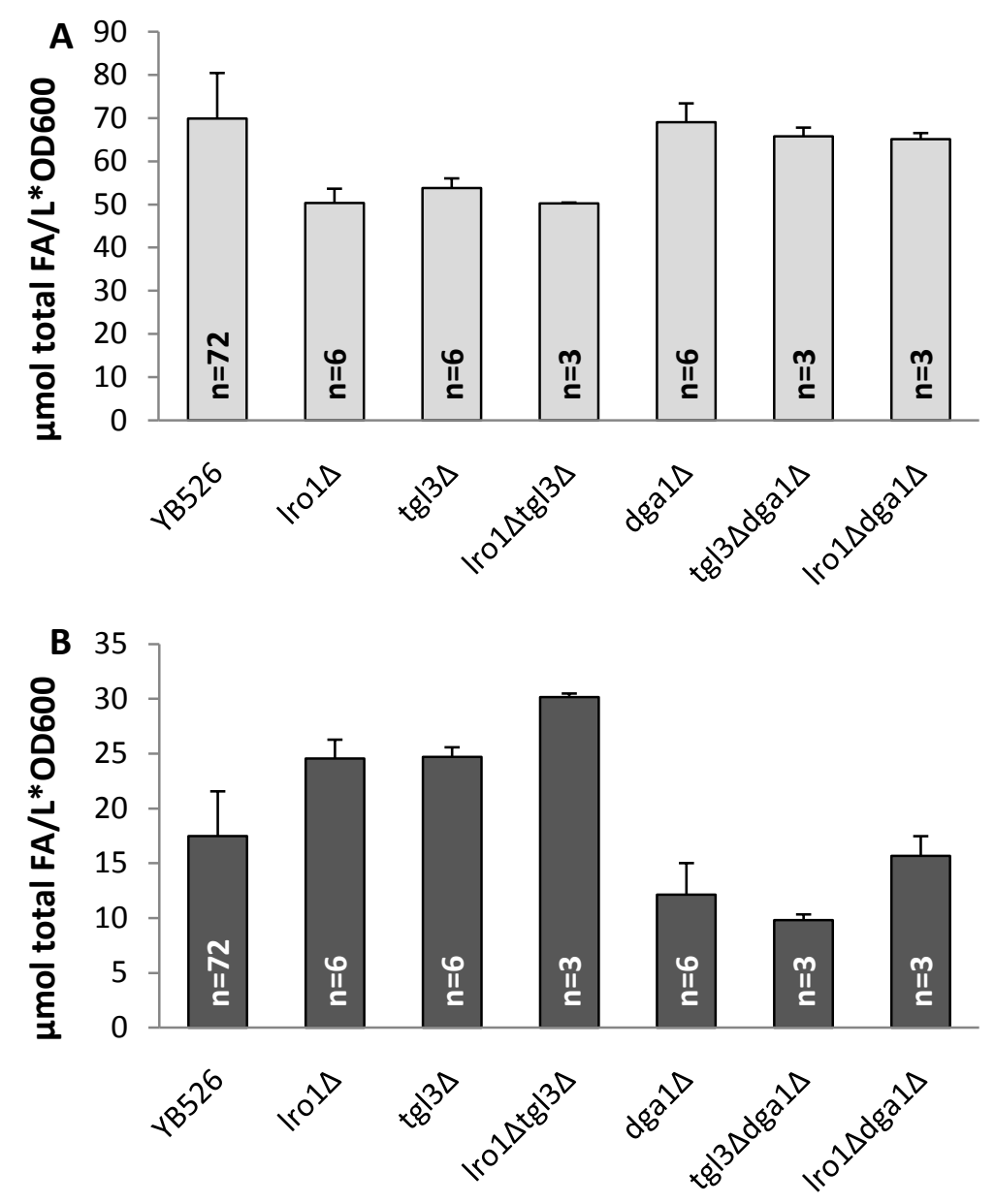

Figure 15. TAG metabolism in FFA production. A: Free FA. In contrast to the PDAT, deletion of the DGAT DGA1 does not lead to a change in the FFA pool size. On the YB526dga1 background the deletion of $L R O 1$ loses its effect. In contrast to its effect on YB526 cells, deletion of TGL3 does not modify the FFA content of

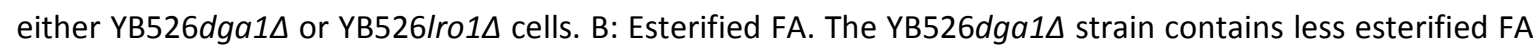
than the reference strain; additional deletion of TGL3 does not reverse this phenotype but deletion of $L R O 1$ does. 
As will be seen in section 5.1.3., the behavior of the free and esterified FA pools in DGA1 deficient strains has lead us to postulate that the rates of PL synthesis and degradation

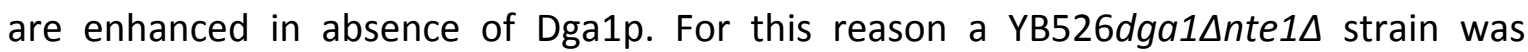
produced. The FFA content of this strain is in fact lower than that of the YB526dga1A (figure 16) despite the fact that the YB526nte1D strain has as much FFA as the YB526 (figure 12). A YB526/ro1 $\Delta$ nte1A strain was also produced. In this LRO1 deficient background, however, the FFA content is not modified by the deletion of NTE1 (figure 16).
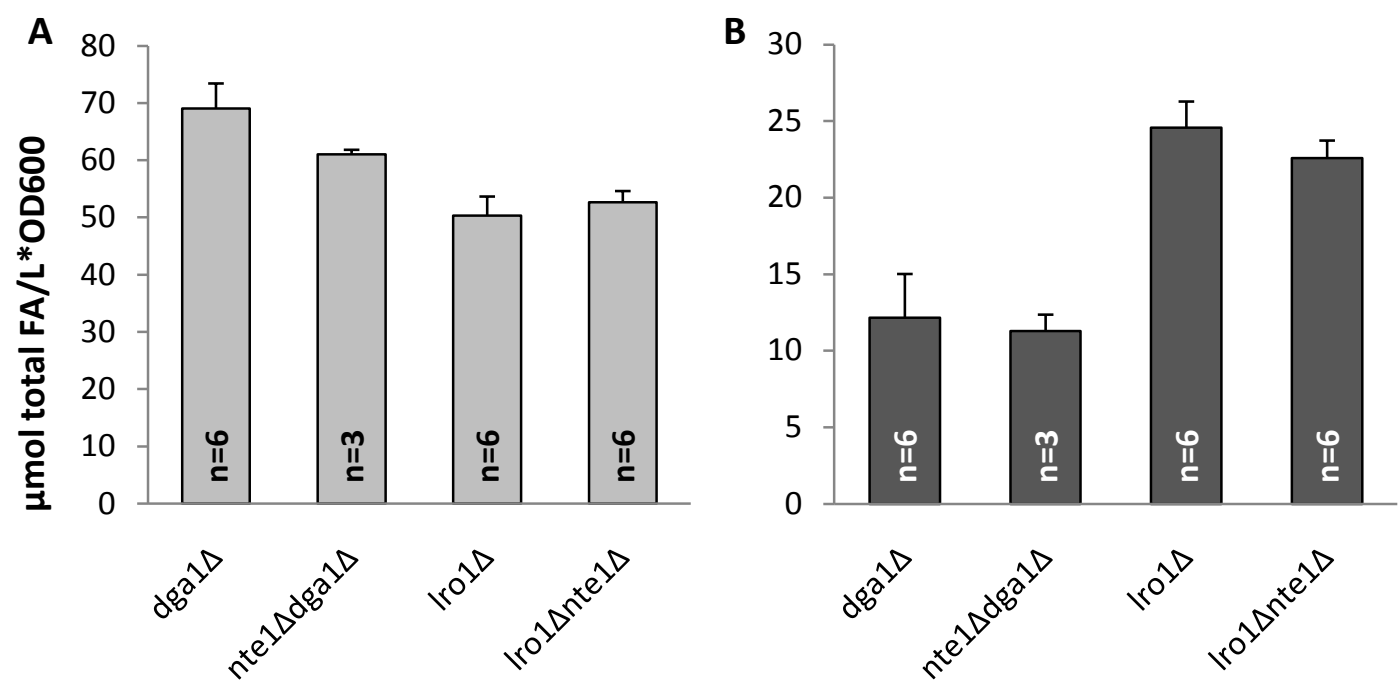

Figure 16. Deletion of NTE1 on YB526dga1D and YB526/ro1 background. On the DGA1 deficient background, but not on the LRO1 deficient background, the deletion of NTE1 causes a decrease in FFA content (A). For esterified FA (B) the opposite effect was observed.

In order to establish whether, similar to TAG metabolism, STE metabolism is also a relevant contributor to the FFA pool, the genes coding for the sterol acyltransferases Are $1 p$ and Are $2 p$, as well as the genes coding for the STE hydrolases Tgl1p, Yeh1p and Yeh2p, were deleted on YB526 cells. In both cases the deletions introduced result in a reduced FFA pool (figure 17). 

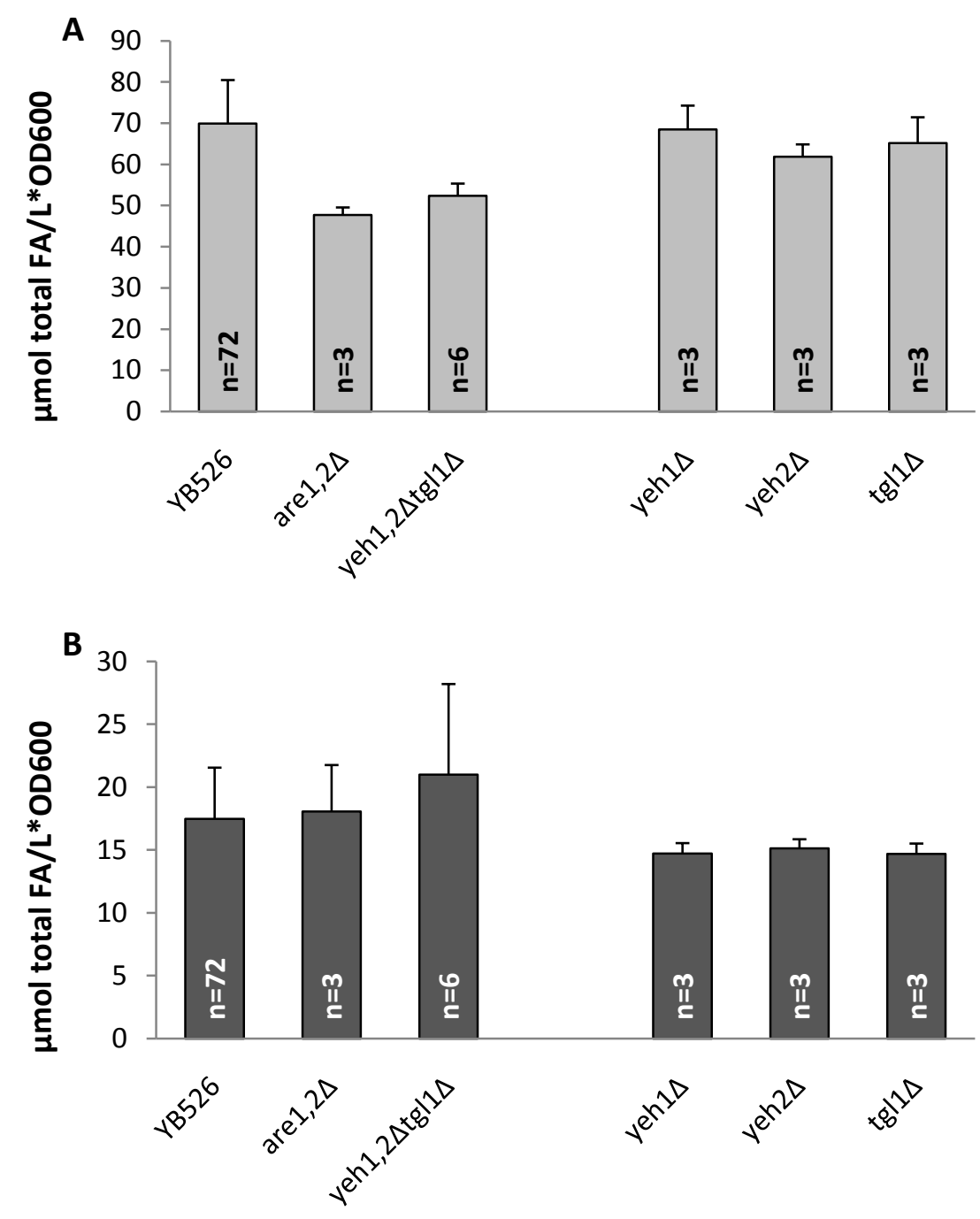

Figure 17. Free (A) and bound (B) FA in STE synthase and hydrolase deficient cells. Interference with STE

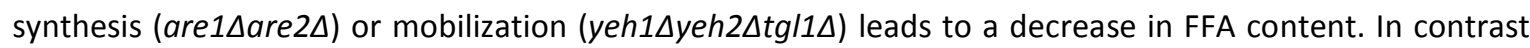
to TAG lipases, the deletion of all STE lipases is necessary to generate the phenotype.

In the next step, mutants simultaneously deficient in both TAG and STE metabolism were

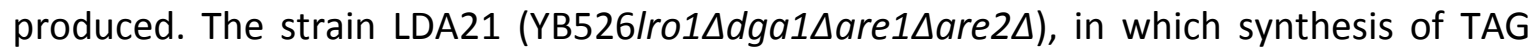

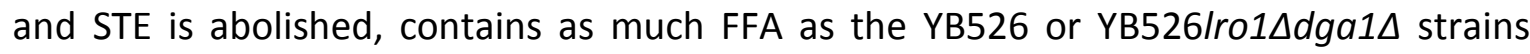
(figure 18). This clearly resembles the situation encountered upon the deletions of LRO1 or TGL3, which affect the FFA content of YB526 cells but not of YB526dga1A cells (figure 15). Simultaneous deletion of the TAG lipase TGL3 and the STE hydrolases YEH1, YEH2 and TGL1, causes a reduction in the size of the FFA pool corresponding to the addition of the reductions caused by independently blocking TAG or STE mobilization (figure 18). 

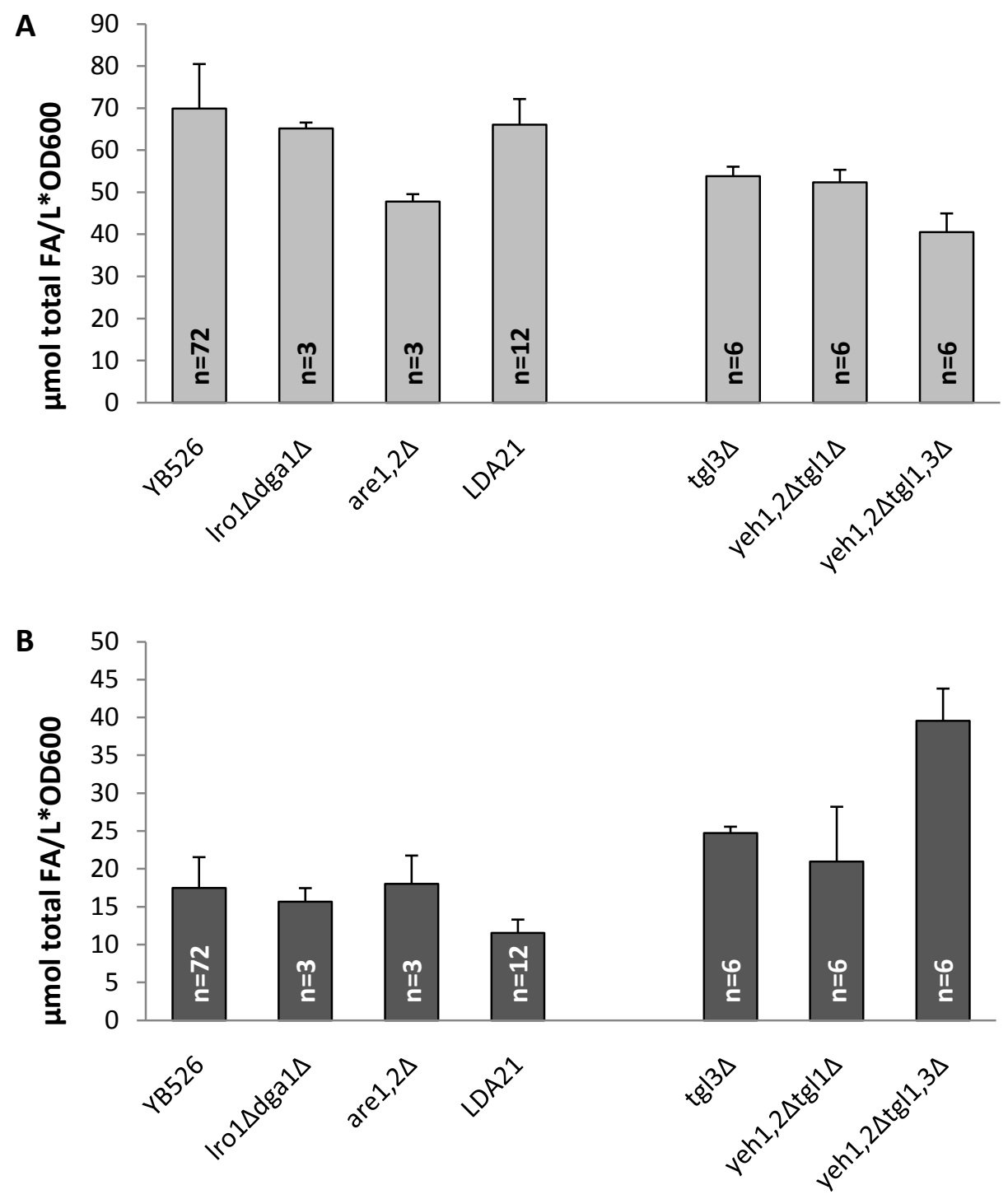

Figure 18. Simultaneous interference with TAG and STE metabolism. In contrast to their effect on the plain YB526 background, deletion of the STE synthases ARE1 and ARE2 on the YB526/ro1 $\triangle$ dga1D background does not decrease the FFA content (A) but leads to lower bound FA content (B). In contrast to the effects of impaired synthesis, the contributions of impaired TAG and STE mobilization to the size of the

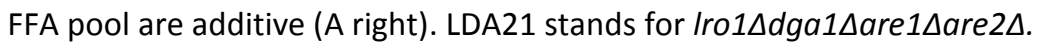

\section{Phospholipase D and lysophospholipid acyltransferases}

Although phospholipases $C$ and D do not directly release FFA, initial degradation of PL by enzymes with these activities could be followed by deacylation of the DAG and PA formed by them, and were therefore considered as possible contributors to the formation of the FFA pool. However, deletion of the PLD SPO14 does not decrease, but 
actually increases the FFA content of YB526 cells (figure 19). Measurements with PLC1 deficient cells require a different technical approach and are therefore presented separately in section 4.3.2.

LPLAT enzymes are responsible for lysolipid acylation; however, some of them have been reported to catalyze the reverse reaction as well, releasing acyl-CoA from a PL. Acyl-CoA could then be converted to FFA by a thioesterase. Deletion of the LPLATS ALE1 and TAZ1, however, does not reduce the FFA content of YB526 cells. Instead, as in the case of SPO14, these deletions result in a higher FFA content (figure 19). The increased production of FFA in these mutants suggests an enhanced rate of PL turnover; in order to test this, NTE1 was deleted on the YB526ale1 $\triangle$ background. Deletion of NTE1 on the ALE1 deficient background does in fact lead to a decrease in FFA content and an increase in the amount of esterified FA (figure 19).
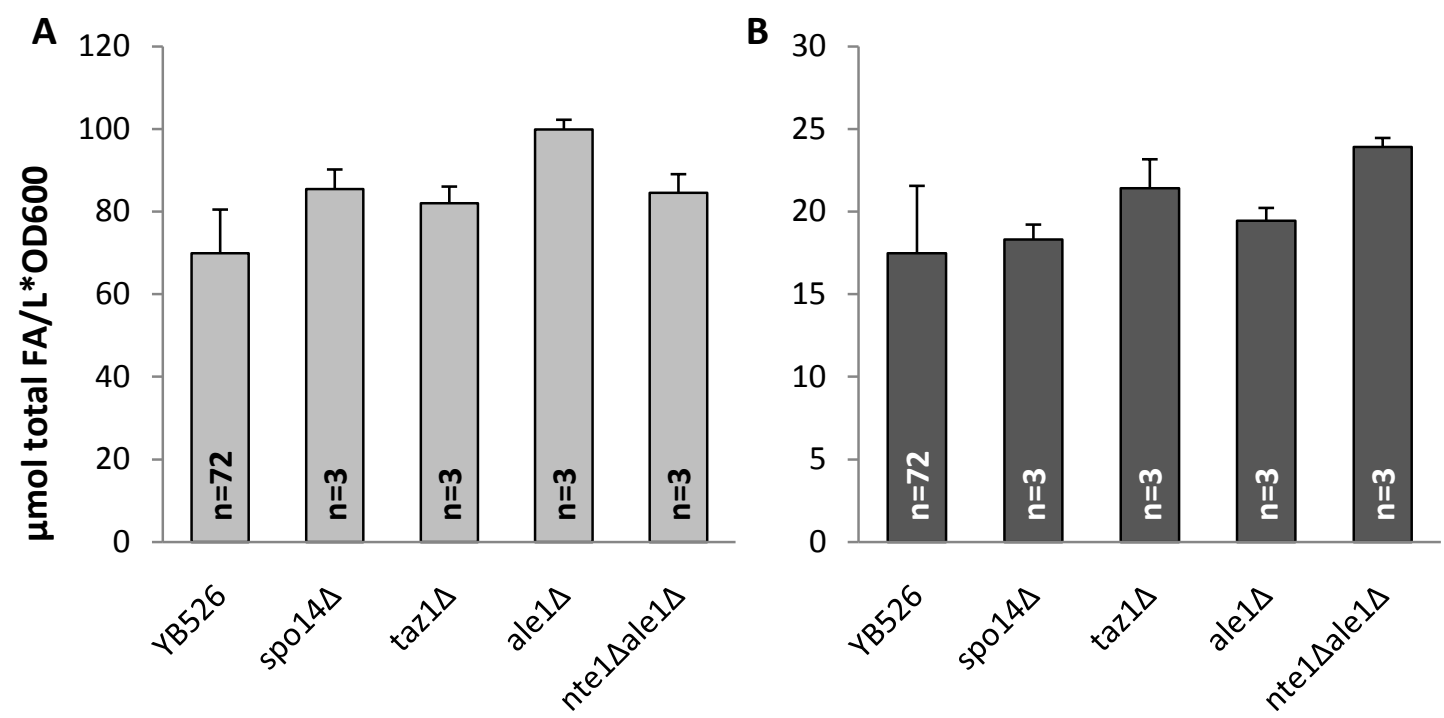

Figure 19. PLD and LPLAT deficient strains. Deletion of the PLD SPO14, or the LPLATS TAZ1 or ALE1 leads to a larger FFA pool (A). The effect is strongest in the YB526ale1 $\triangle$ mutant but is also significant in the other

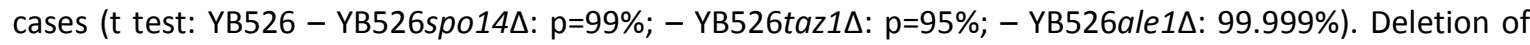
NTE1 on the YB526ale1 $\triangle$ background reduces the FFA content although not to the initial YB526 background

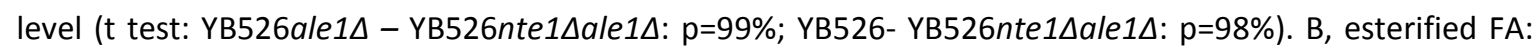
Deletions of SPO14, TAZ1 or ALE1 have no effect on the bound FA content ( $t$ test: YB526 - YB526taz1A: $\mathrm{p}=90 \%)$. Deletion of NTE1 on the YB526ale1 $\triangle$ background leads to a higher esterified FA content (t test:

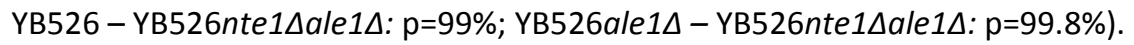




\section{Autophagy}

In order to establish whether or not lipid degradation through autophagy is a relevant contributor to FFA production, YB526 cells deficient for either the formation

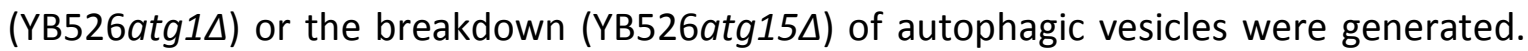
Both conditions lead to a strong reduction of the FFA pool (figure 20, left). Autophagy deficiencies were then combined with those deficiencies of TAG metabolism that

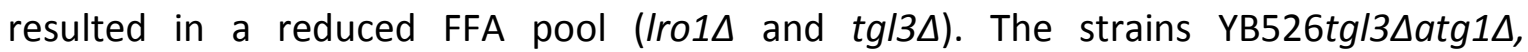

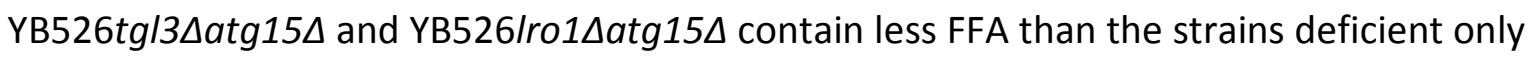
for autophagy or only for TAG metabolism, indicating that these two pathways for FFA formation are additive and independent from each other (figure 20, right). 

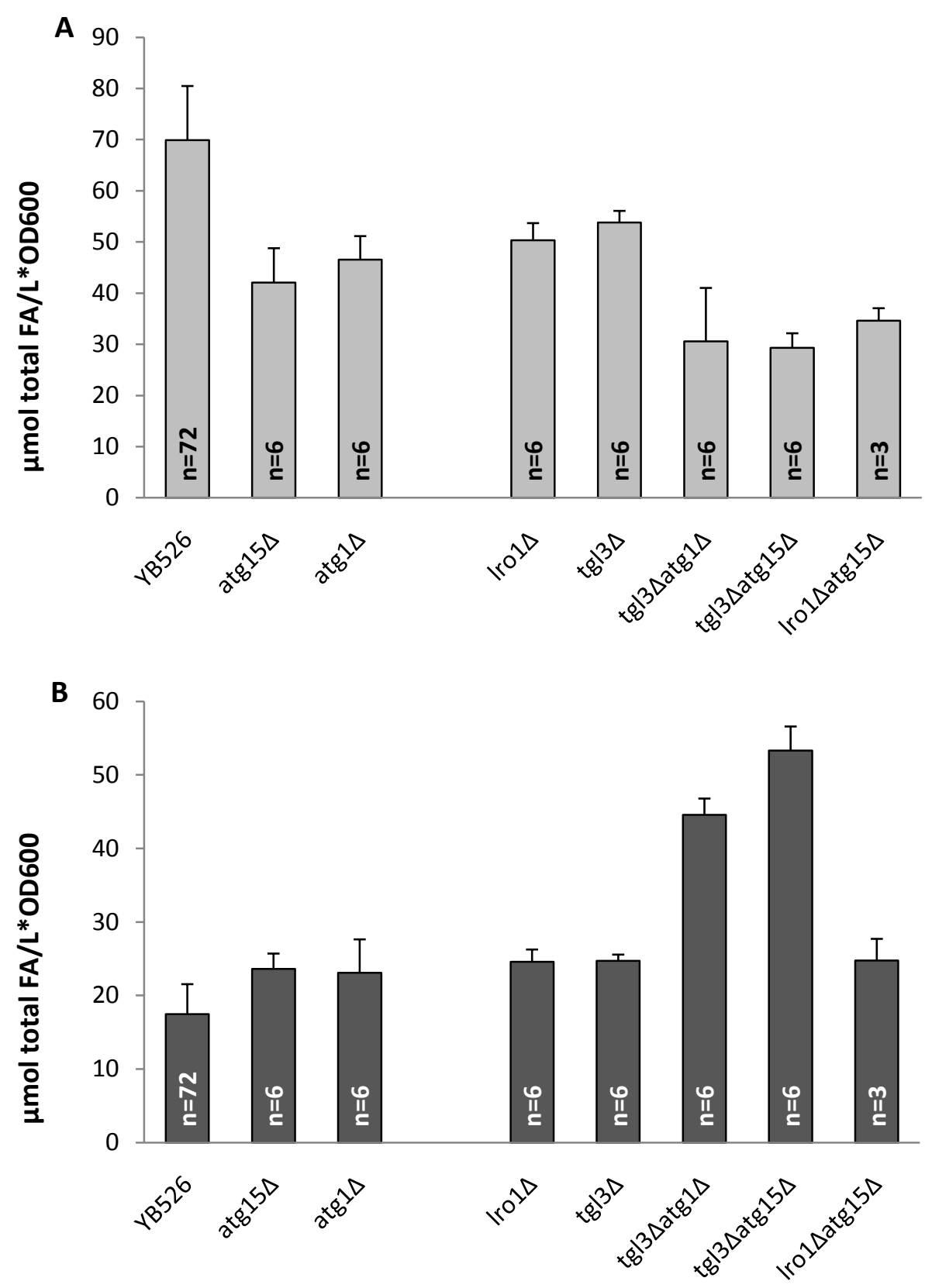

Figure 20. Free (A) and esterified (B) FA in autophagy deficient strains. Blocking autophagic sequestration or degradation has a strong impact on the size of the FFA pool (A left). This decrease in FFA content is additive to the ones caused by LRO1 or TGL3 deletions (A right). FFA decrease in YB526atg1 $\triangle$ and

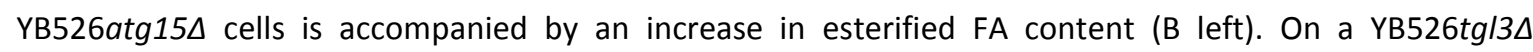
background deletion of $A T G 15$ or ATG1 causes a more than additive increase in esterified FA content; this is not the case for ATG15 deletion on a YB526/ro1 $\triangle$ background (B right). 
ATG1 and ATG15 were then deleted in cells unable to synthesize any NL (LDA21:

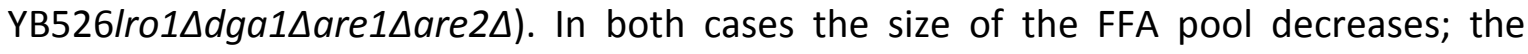
reduction in FFA content is stronger upon deletion of ATG15 than upon deletion of ATG1.
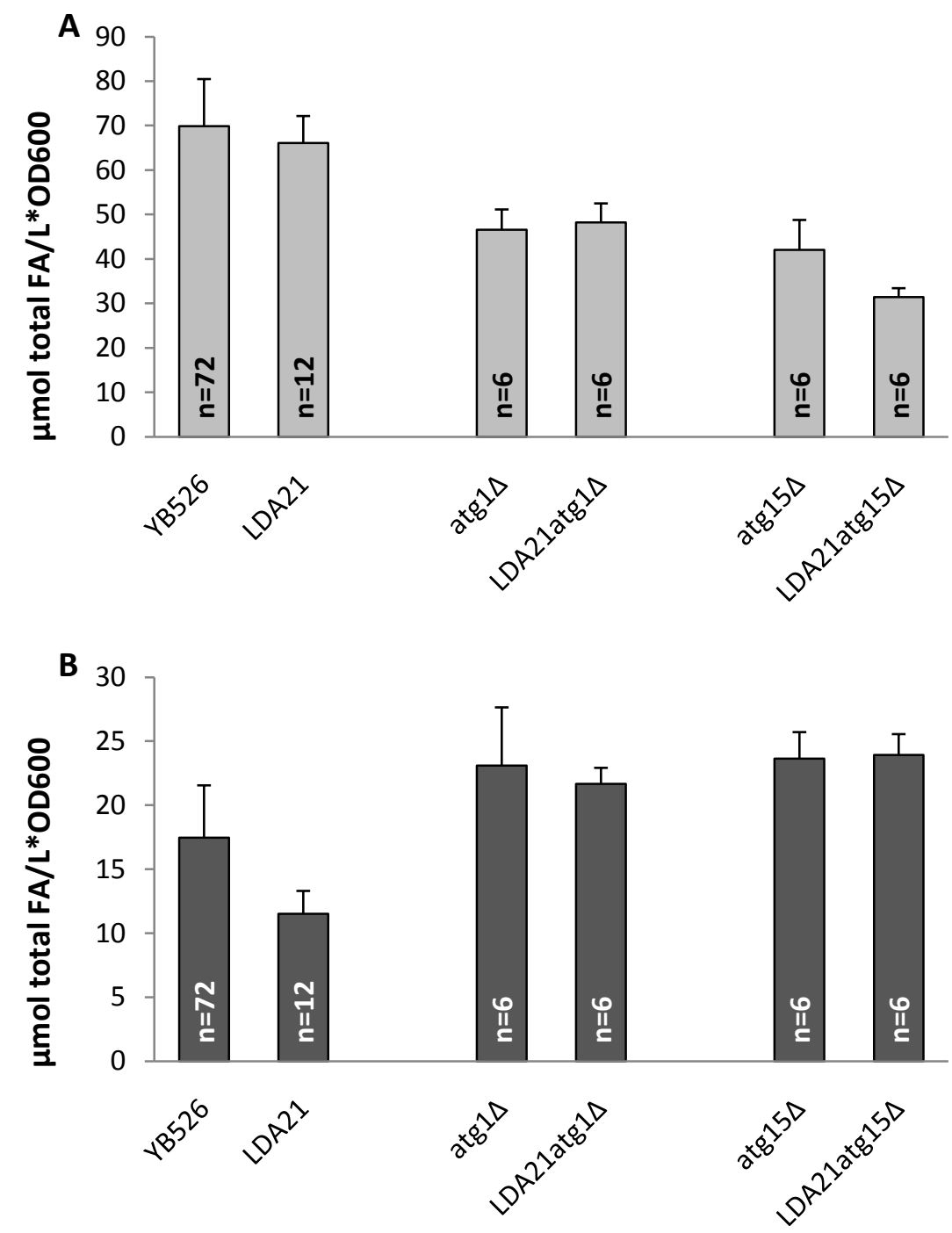

Figure 21. Autophagy in NL devoid strains. Deletion of ATG1 has the same effect on the size of the FFA pool in cells with or without NL (A center); deletion of ATG15, instead, causes a greater reduction in the FFA content of cells unable to make NL (A right). Deletion of ATG1 or ATG15 results in the same esterified FA content in cells with or without NL (B center and right); since NL devoid cells have less esterified FA than the background strain (B left), this implies that impaired autophagy leads to a higher accumulation of PL in cells unable to synthesize NL. 
Although deletion of the TAG lipases TGL4 and TGL5 does not affect the FFA content of YB526 cells (figure 14), it was decided to verify whether they contribute to FFA formation

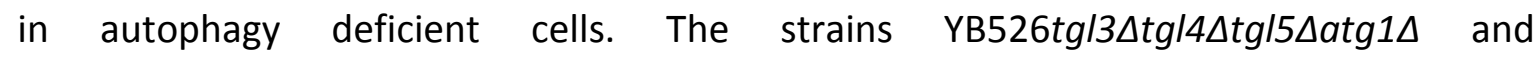

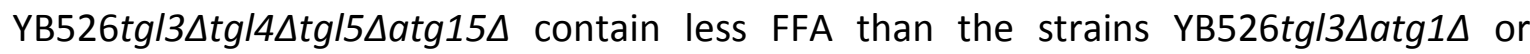
YB526tgl3 $\Delta a t g 15 \Delta$, the differences, however, are very small (figure 22).

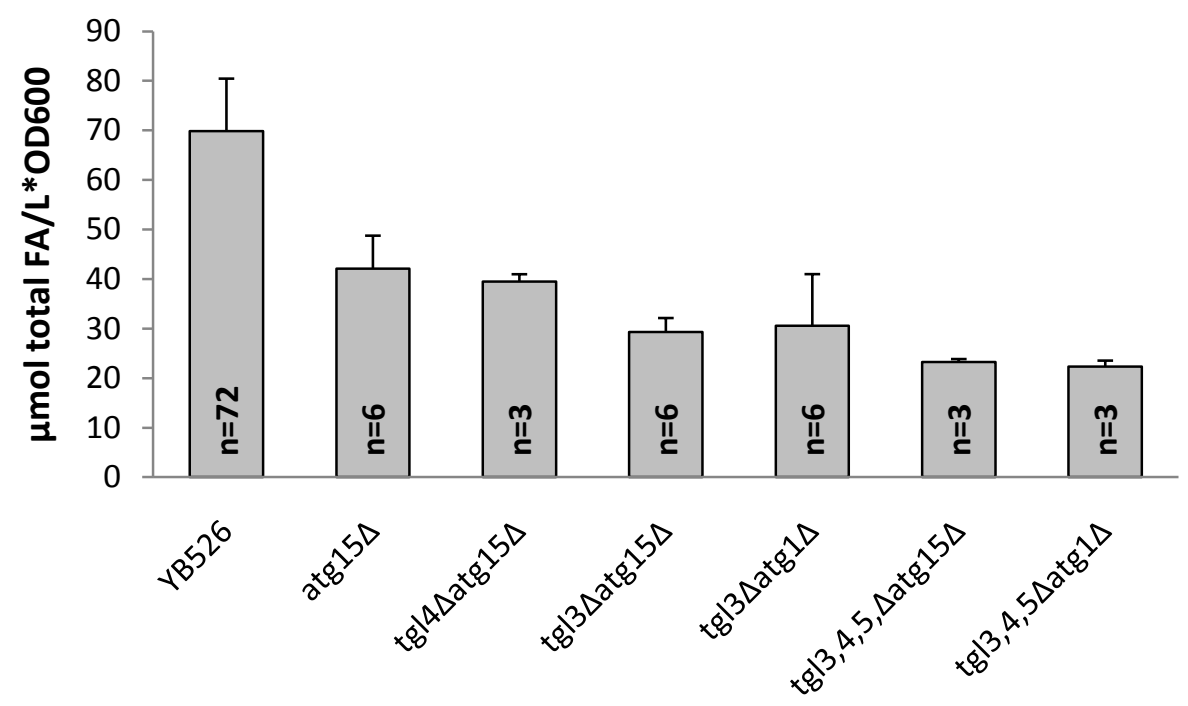

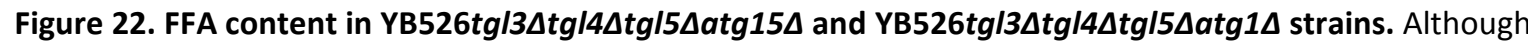
deletion of TGL4 and TGL5 has no effect on the FFA pool size of YB526 or YB526tg/3D cells, their deletion

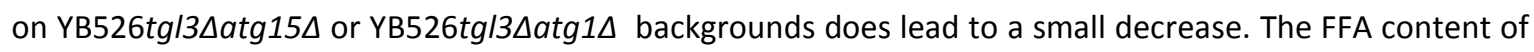

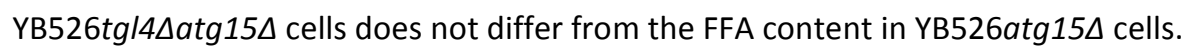




\section{Deletion of peroxisomal proteins}

The gene LPX1 codes for a peroxisomal protein of unidentified function with lipase activity. In order to investigate whether Lpx1p is involved in the formation of the FFA pool, the gene was deleted in YB526 cells and YB526 cells with deficiencies in autophagy or NL metabolism. Deletion of $L P X 1$ causes a considerable decrease in the FFA content of YB526 cells. This effect is not additive to the decreases caused by the deletions of TGL3, ATG15 or ATG1. Absence of $L P X 1$ has no effect on the esterified FA content of YB526,

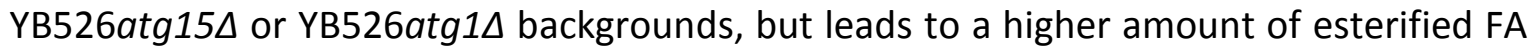

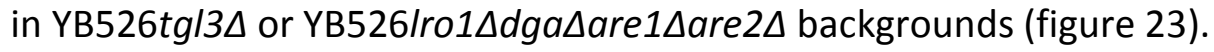
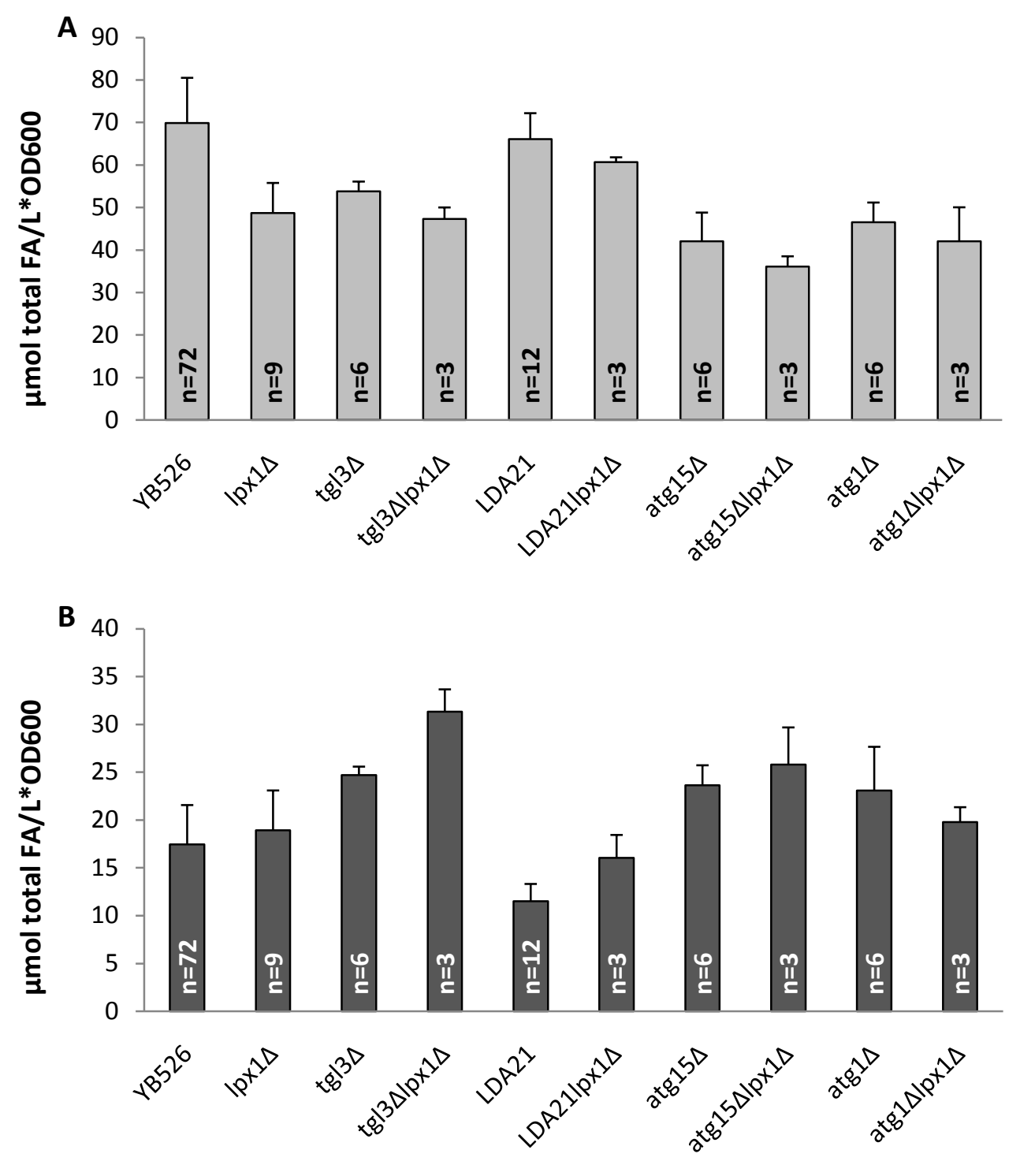

Figure 23. Free (A) and bound (B) FA in LPX1 deficient strains. 
To gain further insight into the role of peroxisomal function in FFA production, PEX3, a gene which's absence prevents peroxisome formation, was deleted in YB526 cells and YB526 cells with deficiencies in autophagy or NL metabolism. Deletion of PEX3 has the same effect on the FFA pool of YB526 than the deletion of LPX1. This reduction in FFA content is not additive to the reductions caused by deletions of TGL3, ATG1, or ATG15. In contrast to LPX1, the effect of PEX3 deletion is also present in cells unable to synthesize NL. Deletion of PEX3 causes a small increase in the esterified FA content of YB526 cells. It also causes a considerable increase of esterified FA content in cells of LDA21,

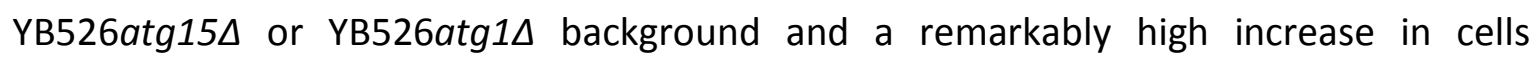
additionally deficient for TGL3 (figure 24).
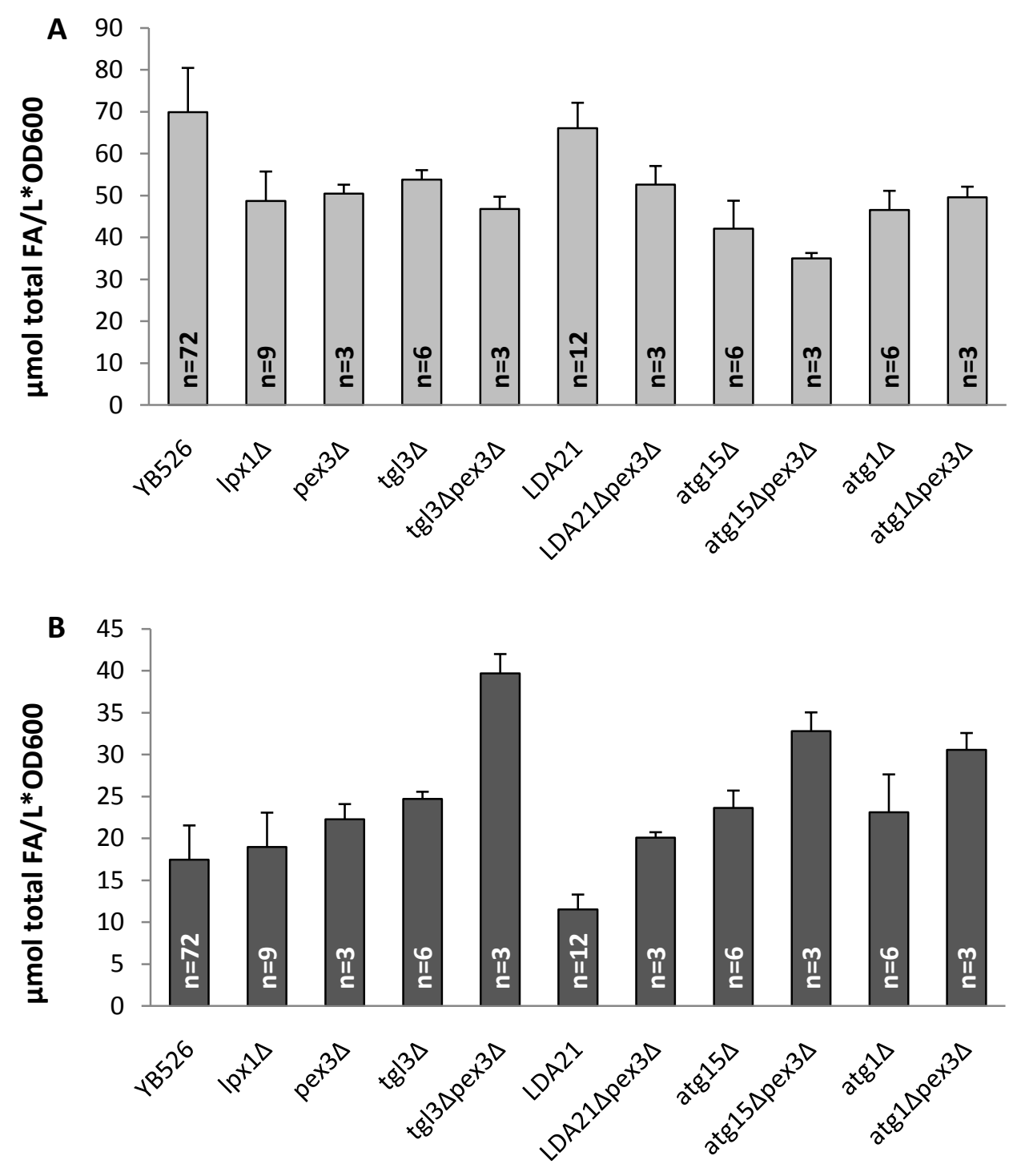

Figure 24. Free (A) and bound (B) FA in PEX3 deficient strains. 
Figures 12 to 24 present only the total FA content of the various mutant strains. The corresponding FA species profiles are presented in table 2. This table displays the relative FA composition of the free and esterified FA pools, i.e. the percentage that each FA species represents within the total FA in the pool and therefore does not indicate absolute changes in the content of a particular species. For example, 16:0 represents $30 \%$ of the FFA in YB526 cells and 31\% of the FFA in YB526tg/3D cells, indicating a small relative increase. However, the absolute content of free 16:0 decreases from $21 \mu \mathrm{mol} / \mathrm{L}^{*} \mathrm{OD}_{600}$ in YB526 cells to $17 \mu \mathrm{mol} / \mathrm{L}^{*} \mathrm{OD}_{600}$ in YB526tg/3 $\mathrm{cells}$. Absolute amounts are presented in appendix 3.

Table 2. Relative composition of the esterified and free fatty acid pools. The values presented correspond to the percentage each FA species represents within the total FA content in the pool. The minor species 14:0 and 18:1(11Z) are not presented in the table but are included in the total.

\begin{tabular}{|c|c|c|c|c|c|c|c|c|c|}
\hline \multirow[b]{2}{*}{ Lane } & & \multicolumn{4}{|c|}{ Esterified FA } & \multicolumn{4}{|c|}{ Free FA } \\
\hline & & $16: 0$ & $16: 1$ & $18: 0$ & 18:1(9Z) & $16: 0$ & $16: 1$ & $18: 0$ & $18: 1(9 Z)$ \\
\hline 1 & YB332 & 13 & 47 & 6 & 31 & 18 & 45 & 12 & 25 \\
\hline 2 & YB526 & 13 & 33 & 9 & 44 & 30 & 26 & 13 & 28 \\
\hline 3 & plb1s & 15 & 31 & 10 & 43 & 33 & 26 & 13 & 26 \\
\hline 4 & plb2s & 13 & 33 & 9 & 43 & 31 & 27 & 13 & 27 \\
\hline 5 & plb3s & 14 & 34 & 8 & 42 & 31 & 27 & 12 & 27 \\
\hline 6 & nte1s & 12 & 32 & 10 & 46 & 31 & 25 & 14 & 28 \\
\hline 7 & $p l b 1,2,3 \Delta$ & 15 & 33 & 9 & 43 & 35 & 25 & 13 & 25 \\
\hline 8 & $\operatorname{tg} / 2 \Delta$ & 15 & 31 & 9 & 44 & 30 & 27 & 13 & 28 \\
\hline 9 & $\operatorname{tg} / 3 \Delta$ & 12 & 34 & 9 & 44 & 31 & 25 & 13 & 28 \\
\hline 10 & $\operatorname{tg} / 4 \Delta$ & 13 & 32 & 9 & 46 & 28 & 27 & 13 & 30 \\
\hline 11 & $\operatorname{tg} 15 \Delta$ & 13 & 33 & 8 & 45 & 30 & 26 & 12 & 28 \\
\hline 12 & $\operatorname{tg} / 3,4,5 \Delta$ & 11 & 37 & 8 & 44 & 29 & 28 & 12 & 28 \\
\hline 13 & Iro1s & 13 & 33 & 8 & 45 & 30 & 29 & 11 & 27 \\
\hline 14 & dga1 $\Delta$ & 15 & 32 & 7 & 45 & 32 & 26 & 13 & 26 \\
\hline 15 & Iro1 $\Delta d g a 1 \Delta$ & 17 & 30 & 8 & 44 & 32 & 27 & 12 & 26 \\
\hline 16 & $|r o 1 \Delta \operatorname{tg}| 3 \Delta$ & 14 & 37 & 8 & 39 & 35 & 25 & 12 & 25 \\
\hline 17 & $\operatorname{tg} / 3 \Delta d g a 1 \Delta$ & 15 & 34 & 8 & 43 & 33 & 26 & 13 & 26 \\
\hline 18 & nte $1 \Delta d g a 1 \Delta$ & 14 & 33 & 9 & 44 & 32 & 25 & 14 & 26 \\
\hline 19 & 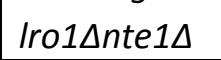 & 13 & 35 & 9 & 42 & 31 & 27 & 13 & 26 \\
\hline
\end{tabular}


Table 2. Continued from previous page.

Esterified FA

Free FA

\begin{tabular}{|c|c|c|c|c|c|c|c|c|c|}
\hline Lane & & $16: 0$ & $16: 1$ & $18: 0$ & $18: 1(9 Z)$ & $16: 0$ & $16: 1$ & $18: 0$ & $18: 1(9 Z)$ \\
\hline 20 & YB526 & 13 & 33 & 9 & 44 & 30 & 26 & 13 & 28 \\
\hline 21 & $\operatorname{are} 1,2 \Delta$ & 12 & 31 & 8 & 48 & 31 & 26 & 14 & 26 \\
\hline 22 & yeh1s & 16 & 30 & 10 & 43 & 36 & 23 & 14 & 24 \\
\hline 23 & yeh2A & 15 & 32 & 9 & 43 & 35 & 23 & 14 & 25 \\
\hline 24 & $\operatorname{tg} / 1 \Delta$ & 15 & 32 & 9 & 43 & 35 & 24 & 14 & 25 \\
\hline 25 & yeh1,2 $2 \operatorname{tg} / 1 \Delta$ & 14 & 32 & 9 & 44 & 34 & 26 & 13 & 25 \\
\hline 26 & LDA21 & 15 & 31 & 7 & 46 & 28 & 26 & 13 & 29 \\
\hline 27 & yeh1,2 $\Delta \operatorname{tg} / 1,3 \Delta$ & 14 & 33 & 10 & 42 & 36 & 25 & 12 & 24 \\
\hline 28 & spo14 & 13 & 30 & 9 & 47 & 29 & 27 & 13 & 30 \\
\hline 29 & $\operatorname{taz} 1 \Delta$ & 13 & 30 & 9 & 48 & 29 & 28 & 12 & 29 \\
\hline 30 & ale1s & 13 & 33 & 8 & 44 & 28 & 30 & 10 & 25 \\
\hline 31 & nte1 $1 \Delta a l e 1 \Delta$ & 11 & 36 & 6 & 44 & 22 & 33 & 9 & 29 \\
\hline 32 & $\operatorname{atg} 15 \Delta$ & 18 & 38 & 8 & 34 & 38 & 21 & 13 & 24 \\
\hline 33 & $\operatorname{atg} 1 \Delta$ & 16 & 34 & 9 & 39 & 37 & 22 & 13 & 25 \\
\hline 34 & $\operatorname{tg} 13 \Delta a \operatorname{tg} 15 \Delta$ & 18 & 38 & 8 & 34 & 50 & 14 & 12 & 18 \\
\hline 35 & $\operatorname{tg} \mid 4 \Delta a \operatorname{tg} 15 \Delta$ & 17 & 38 & 8 & 37 & 39 & 20 & 15 & 22 \\
\hline 36 & $\operatorname{tg} / 3,4,5, \Delta a \operatorname{tg} 15 \Delta$ & 19 & 40 & 8 & 32 & 49 & 16 & 11 & 19 \\
\hline 37 & $\operatorname{tg} / 3 \Delta a \operatorname{tg} 1 \Delta$ & 19 & 35 & 10 & 36 & 47 & 16 & 13 & 21 \\
\hline 38 & $\operatorname{tg} / 3,4,5 \Delta a \operatorname{tg} 1 \Delta$ & 17 & 37 & 9 & 35 & 39 & 20 & 11 & 27 \\
\hline 39 & Iro1 $\Delta a \operatorname{tg} 15 \Delta$ & 16 & 40 & 6 & 36 & 36 & 22 & 12 & 27 \\
\hline 40 & LDA21atg15 & 17 & 37 & 6 & 39 & 44 & 19 & 15 & 18 \\
\hline 41 & LDA21atg1 & 16 & 31 & 7 & 46 & 34 & 23 & 13 & 27 \\
\hline 42 & $\mid p \times 1 \Delta$ & 14 & 31 & 10 & 45 & 32 & 26 & 13 & 27 \\
\hline 43 & $\operatorname{tg} \mid 3 \Delta / p \times 1 \Delta$ & 14 & 34 & 9 & 41 & 36 & 24 & 12 & 25 \\
\hline 44 & LDA21/px1 & 17 & 29 & 7 & 47 & 34 & 24 & 14 & 26 \\
\hline 45 & $\operatorname{atg} 15 \Delta / p \times 1 \Delta$ & 20 & 37 & 7 & 35 & 41 & 21 & 13 & 21 \\
\hline 46 & $\operatorname{atg} 1 \Delta / p \times 1 \Delta$ & 17 & 38 & 8 & 36 & 41 & 19 & 14 & 23 \\
\hline 47 & pex3s & 13 & 37 & 7 & 41 & 29 & 28 & 11 & 30 \\
\hline 48 & $\operatorname{tg} 13 \Delta p e x 3 \Delta$ & 16 & 34 & 10 & 38 & 41 & 20 & 13 & 23 \\
\hline 49 & LDA21 1 pex3 $\triangle$ & 15 & 32 & 6 & 46 & 29 & 27 & 13 & 29 \\
\hline 50 & $\operatorname{atg} 15 \Delta p e x 3 \Delta$ & 22 & 38 & 6 & 32 & 44 & 18 & 13 & 21 \\
\hline 51 & $\operatorname{atg} 1 \Delta p e x 3 \Delta$ & 19 & 35 & 9 & 36 & 40 & 19 & 14 & 24 \\
\hline
\end{tabular}




\subsubsection{Phospholipase $C$}

As mentioned above, the potential role of the phospholipase C Plc1p in lipid deacylation could not be addressed in the exact same way as that of our other candidate proteins. Cells with a null mutation of PLC1 have been reported to present growth defects depending on culture conditions (media composition and temperature) and/or genetic background (Flick and Thorner 1993; Rebecchi and Pentyala 2000; Yoko-o et al. 1993). Our YB526plc1 $\Delta$ cells are viable but grow very poorly (maximum OD $_{600}$ of 1 in YPR media, figure 28), making the comparison of their FFA content to that of the YB526 strain unviable.

Establishing the role of PIc1p in the formation of the FFA pool observed in ACS deficient cells was of particular interest given that Nakayama and coworkers (Nakayama et al. 2002) have concluded that the toxicity encountered upon supplementing yeast cells with $\mathrm{Mg}^{2+}$ and AMPC16 (an inhibitor of ACS activity) is due to enhanced irreversible deacylation of plasma membrane PL mediated by Plc1p. In cells treated with AMPC16, deacylation is irreversible in the same way that in our acyl-CoA synthetase deficient cells: the released FA cannot be reutilized, however the remaining glycerophosphodiesters or lysolipids can, in both cases, be reacylated with de novo synthesized acyl-CoA or through transacylation from another lipid. Nakayama and coworkers propose that the toxicity upon combination of AMPC16 and $\mathrm{Mg}^{2+}$ is derived from an abnormally high level of PL deacylation induced by the presence of the magnesium ions. Furthermore they propose that the target of $\mathrm{Mg}^{2+}$ induction is PLC activity given that, in contrast to the wild type (W303), their W303plc1s strain grows normally in media containing AMPC16 and $\mathrm{Mg}^{2+}$.

In order to bypass the growth defect we constructed ACS deficient cells (YB332faa1 $\Delta$ faa4 4 fat1 1 ) in which PIc1p was modified according to the DEGRON system (Sanchez-Diaz et al. 2004, see sections 3.2.2.7. and 3.2.3.5.). In this experiment a recombinant Plc1p is expressed and functional at low temperatures $\left(28^{\circ} \mathrm{C}\right)$, therefore allowing the cells to grow normally (figure 25). Upon transferring the culture to a higher temperature $\left(37^{\circ} \mathrm{C}\right)$ in presence of galactose, degradation of the recombinant protein is rapidly induced and the evolution of the FFA pool, now in absence of Plc1p, can be evaluated. Cells carrying the same deletions but with a wild type PLC1 gene where grown in parallel and treated identically. Throughout $25 \mathrm{~h}$ after the degradation of the 
recombinant Plc1p was induced, the FFA pool continued to increase and reached the same level in both cultures (figure 25). This result indicates that Plc1p is not indispensable for FFA production and that, at least during late exponential / early stationary phase, it makes no significant contribution to FFA formation.
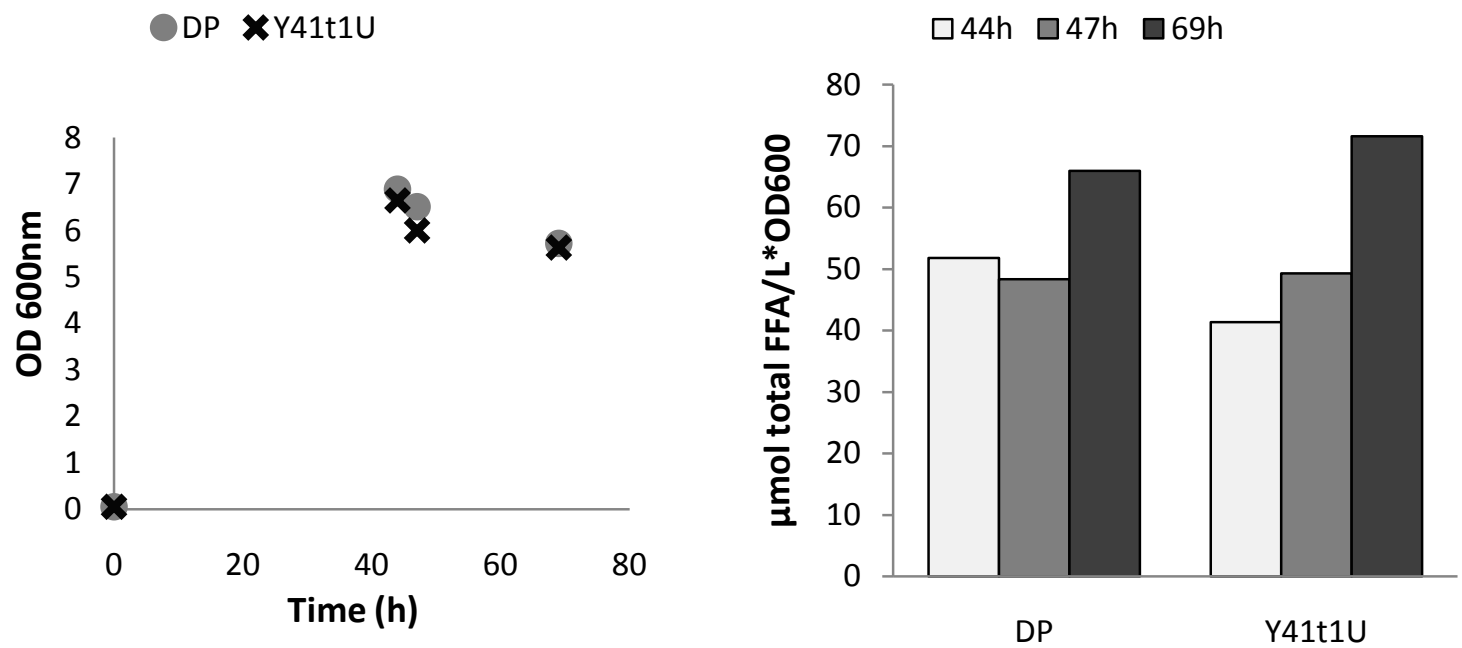

Figure 25. Heat inducible PIc1p degradation. Strain Y41t1U: YB332faa1 4 faa4 $\triangle f a t 1 \Delta$, UBR1 inserted for expression under GAL1 promoter and wild type PLC1 controlled by the native PLC1 promoter. Strain DP: Y41t1U cells with PLC1 promoter replaced by the copper inducible CUP1 promoter and the heat inducible degron cassette additionally introduced at the start of the PLC1 ORF.

The cells where grown at $24^{\circ} \mathrm{C}$ in YPR with $0.1 \mathrm{mM} \mathrm{CuSO}_{4}$ for 44 hours. Under these conditions the fusion degron-Plc1 protein is stable and no growth defect appears (left). At $44 \mathrm{~h}$ a sample for OD and FA determination was taken, galactose was added and cells where transferred to $37^{\circ} \mathrm{C}$, thereby inducing the degradation of the degron-PIc1 fusion protein in the DP strain but not of the wt PIc1p in the Y41t1U strain. 25 hours later ( $69 \mathrm{~h}$ ) the FFA content in the culture without PIc1p has increased to approximately the same level as in the culture with PIc1p (right). The difference is $7 \%$. In this case we have no measurement of standard deviation but we know from measurements in other strains that changes in FFA content beneath $10 \%$ are generally not significant within the sensitivity of our system.

Given that this result contradicts the hypothesis of Nakayama and coworkers, we decided to test their observations within our experimental model. YB526fat1 $\Delta$ cells where grown with and without addition of $\mathrm{Mg}^{2+}$. The deletion of acyl-CoA synthetases should be equivalent to the addition of the inhibitor, however, in contrast to their report, we found 
no growth defect (figure 26). We also found no variation in the content of FFA caused by the presence of $\mathrm{Mg}^{2+}$ (figure 27).

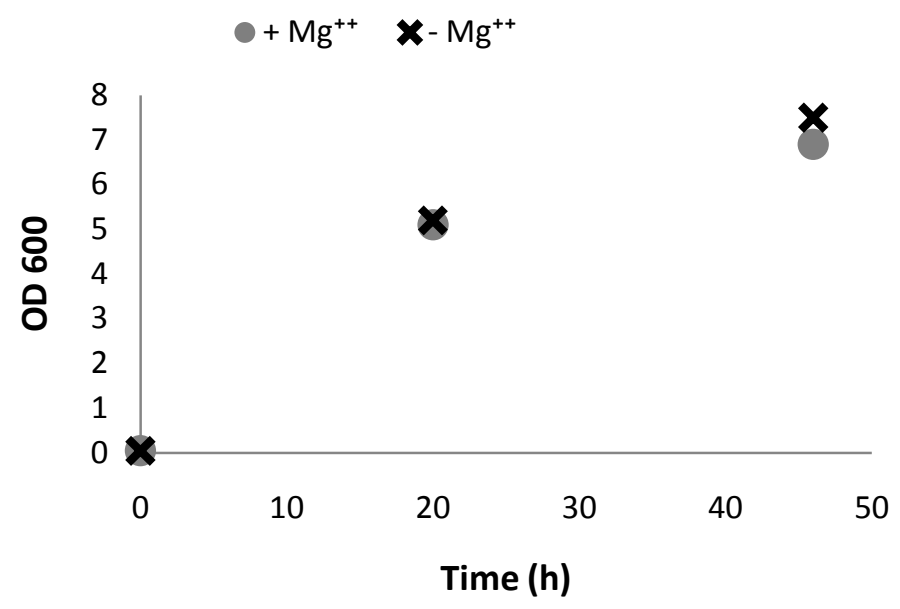

Figure 26. Growth curve with and without $\mathrm{Mg}^{2+}$. ACS deficient cells YB526fat1 $\triangle$ were grown in YPD with or without the addition of $\mathrm{MgSO}_{4}$ to $10 \mathrm{mM}$. No growth defect was found.

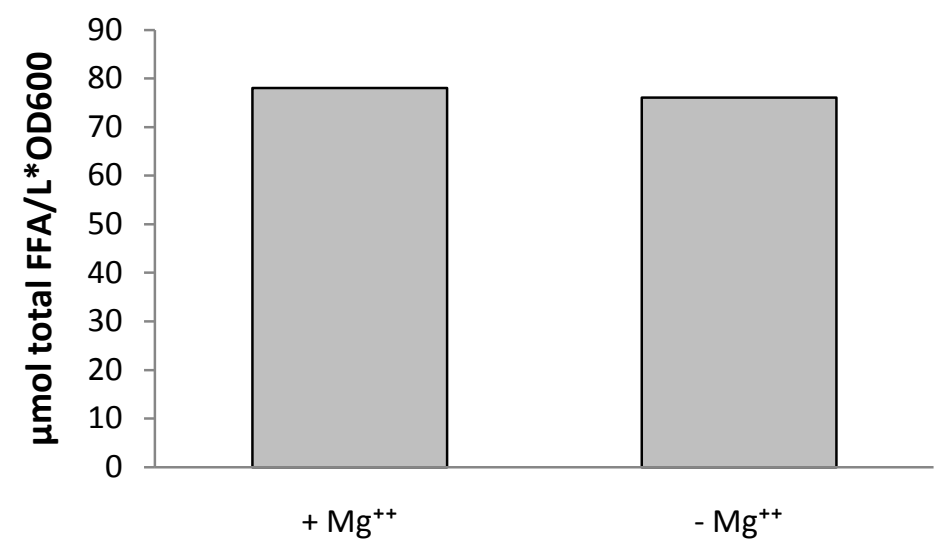

Figure 27. FFA content of YB526fat1 cells with and without $\mathrm{Mg}^{2+}$. Cells where grown for $46 \mathrm{~h}$ in YPD media with or without $10 \mathrm{mM} \mathrm{MgSO}_{4}$. There is no difference in the size of the FFA pool.

Once the role of PIc1p had been addressed in this rather circumventing way, it was found that, in a different genetic background (BY4741 instead of our usual YB332), the combined deletion of acyl-CoA synthetases and PLC1 does not lead to a growth defect. BY4741faa1 1 faa4 $\Delta$ plc1 1 cells grow normally (figure 28 , left) and their FFA content after 
$136 \mathrm{~h}$ of culture is not significantly different to that of YB526 cells (figure 28, right). It was decided to present the results obtained by both methods since, given the entirely different experimental setup in the first case and the different genetic background in the second, neither of them is, alone, completely equivalent to the results presented in figures 11 to 24 .
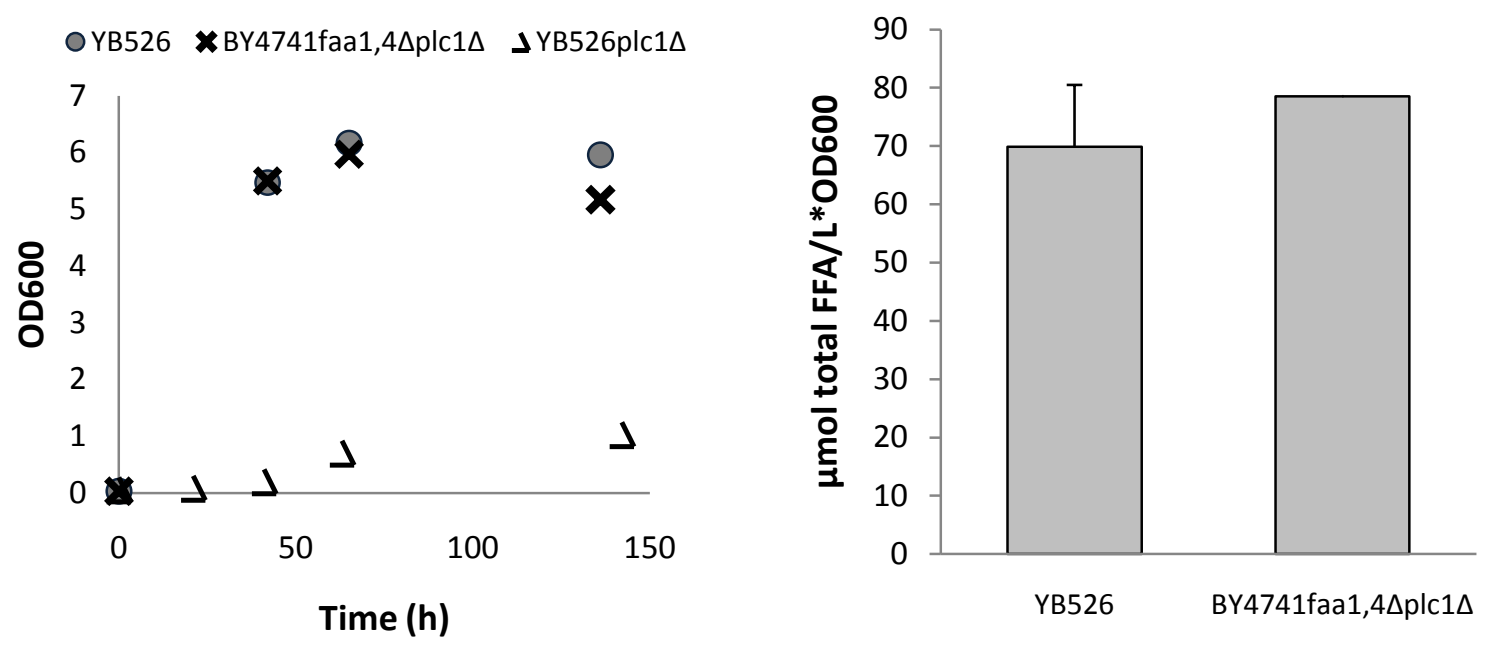

Figure 28. PLC1 deletion on BY4741 cells. In contrast to YB526plc1 1 cells which fail to reach an $\mathrm{OD}_{600}$

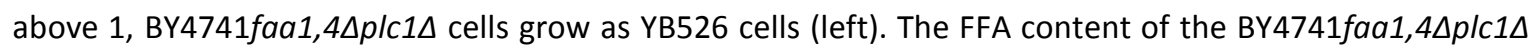
strain after 136h of growth in YPR media is the same of YB526 cells (right).

\subsection{Quantification of FA in specific lipid classes}

\subsubsection{FA in TAG}

As indicated before, the quantifications of esterified FA so far presented correspond to FA in all lipid classes together. In order to gain further insight into the relation between TAG metabolism and autophagy, revealed by the results presented in figures 20 and 21, the content of FA specifically esterified in TAG was quantified for some strains carrying deletions of TGL3, ATG1 and ATG15. The results of those measurements are presented in figure 29. A strain carrying a deletion of $L P X 1$ was also included in the experiment in order to establish whether absence of this gene has an effect on TAG content. The cultures utilized for these measurements were grown under the exact same conditions as those used for the quantification of FFA and total esterified FA presented in section 4.3.1. Some further measurements of FA in TAG and other specific lipid classes were carried 
out, but employing cultures grown under different conditions; those results are therefore presented separately in section 4.4.2.

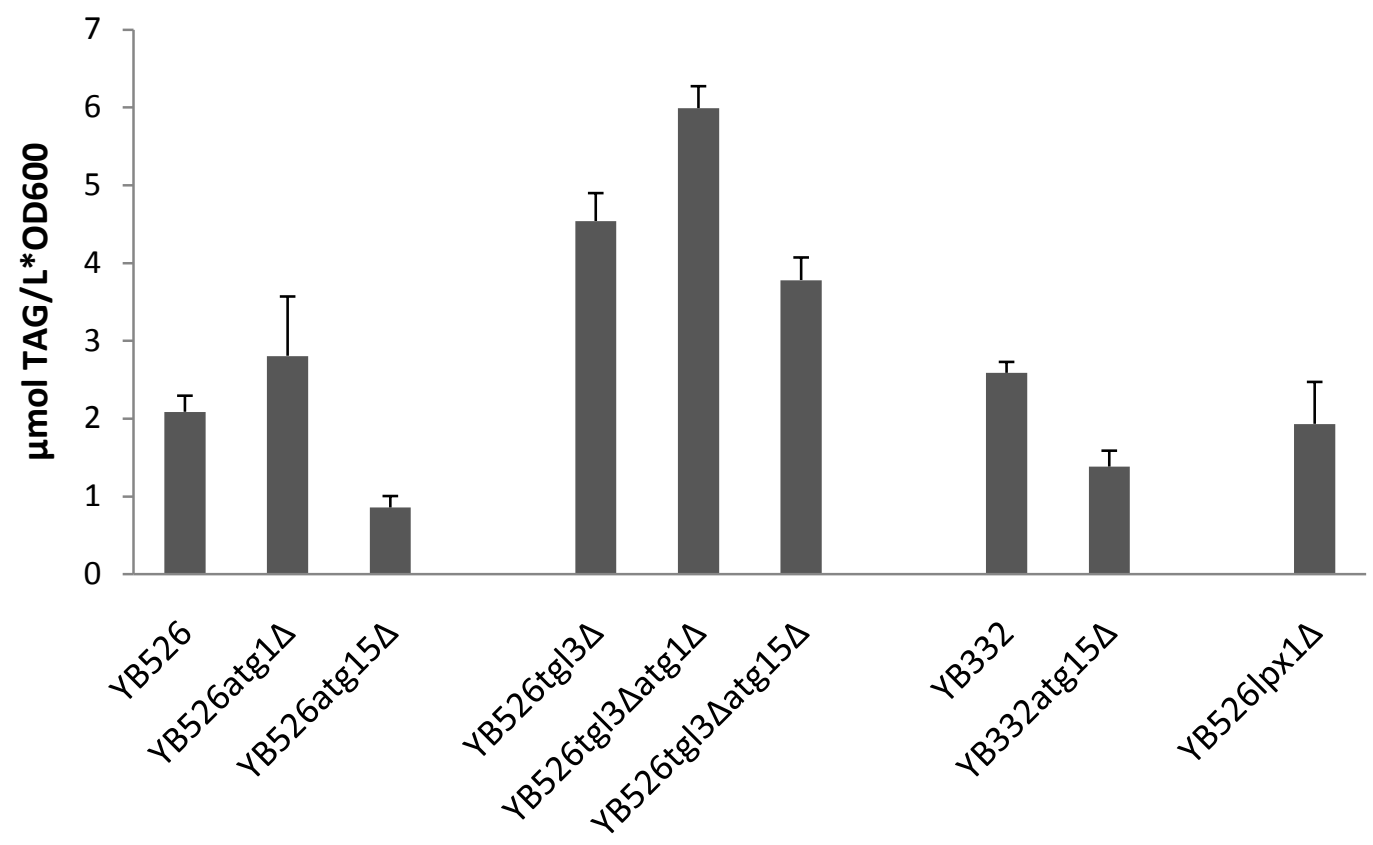

Figure 29. TAG content at late stationary phase $(136 \mathrm{~h})$. Deletion of ATG1 leads to an increase of TAG content both on YB526 and YB526tgl3A backgrounds. Deletion of ATG15, in contrast, results in a decrease of TAG content on these two backgrounds as well as on a wild type (YB332) background. Deletion of LPX1 has no effect on the amount of TAG in YB526 cells. Error bars correspond to standard deviation within three replica cultures derived from a single preculture.

Table 3. Relative FA composition in TAG. The values presented correspond to the percentage each FA species represents within the total FA present in TAG. The minor species 14:0 and 18:1(11Z) are not presented in the table but are included in the total.

\begin{tabular}{|c|c|c|c|c|}
\hline & $16: 0$ & $16: 1$ & $18: 0$ & 18:1(9Z) \\
\hline YB526 & 12 & 30 & 12 & 45 \\
\hline YB526atg1 $\Delta$ & 14 & 35 & 10 & 39 \\
\hline YB526atg15 $\Delta$ & 15 & 35 & 8 & 40 \\
\hline YB526tgl3 $\Delta$ & 13 & 32 & 12 & 42 \\
\hline YB526tgl3 $\Delta$ atg1 $\Delta$ & 15 & 37 & 11 & 35 \\
\hline YB526tgl3 $\Delta$ atg15 $\Delta$ & 15 & 37 & 12 & 34 \\
\hline YB332 & 10 & 50 & 4 & 33 \\
\hline YB332atg15 $\Delta$ & 13 & 56 & 3 & 24 \\
\hline YB526lpx1 $\Delta$ & 14 & 32 & 11 & 42 \\
\hline
\end{tabular}




\subsubsection{FA in various lipid classes}

As mentioned in section 4.3.1., the present work is mainly concerned with identifying the elements of the metabolic network for lipid, and in particular phospholipid, deacylation as well as the connections between these elements. The approach we use is essentially a steady state one, as we analyze the effect of genetic conditions on the final (stationary phase) equilibrium of different lipid pools. The natural next step will be an analysis of lipid deacylation and FA transit through the metabolic network in a dynamic way; such study would be enormously favored by the use of labeled FA as their progression through the network could be followed. Given the complexity of the network and the difficulty in identifying its components and their connections, the dynamic analysis of FA flow falls beyond the scope of this work; nevertheless we carried out several preliminary tests on the viability of some methods for the introduction of label into the system. In our strains this is a challenging task, since in absence of any fatty acid activation capacity it is impossible to introduce label into the fatty acid metabolism by just supplementing the cells with free fatty acids. Therefore, the following methods were employed: Uptake of lysoPC with a labeled acyl chain, the expression of an exogenous desaturase which converts 18:1 FA into 18:2 (a species otherwise absent in yeast), and the transient expression of a degradable acyl-CoA synthetase which allows incorporation of labeled FA followed by a return to the acyl-CoA synthetase deficient status. The last approach was explored within a master's thesis carried out as a satellite work of the present one and the results are described in it (Jessen 2008). The results of the first and second approach will not be described here in detail as the tests were truly preliminary and most importantly were concerned with the viability of introducing labeled FA rather than a careful chase of the label and would therefore not contribute in the discussion to follow. However, within our attempt to verify the distribution of 18:2 a quantification of all FA in several specific lipid classes was performed for a subset of mutant strains. Beyond the behavior of the 18:2 FA, that result is of relevance in the general discussion of the effect that the introduced mutations have on lipid homeostasis and is therefore presented in figure 30 . 

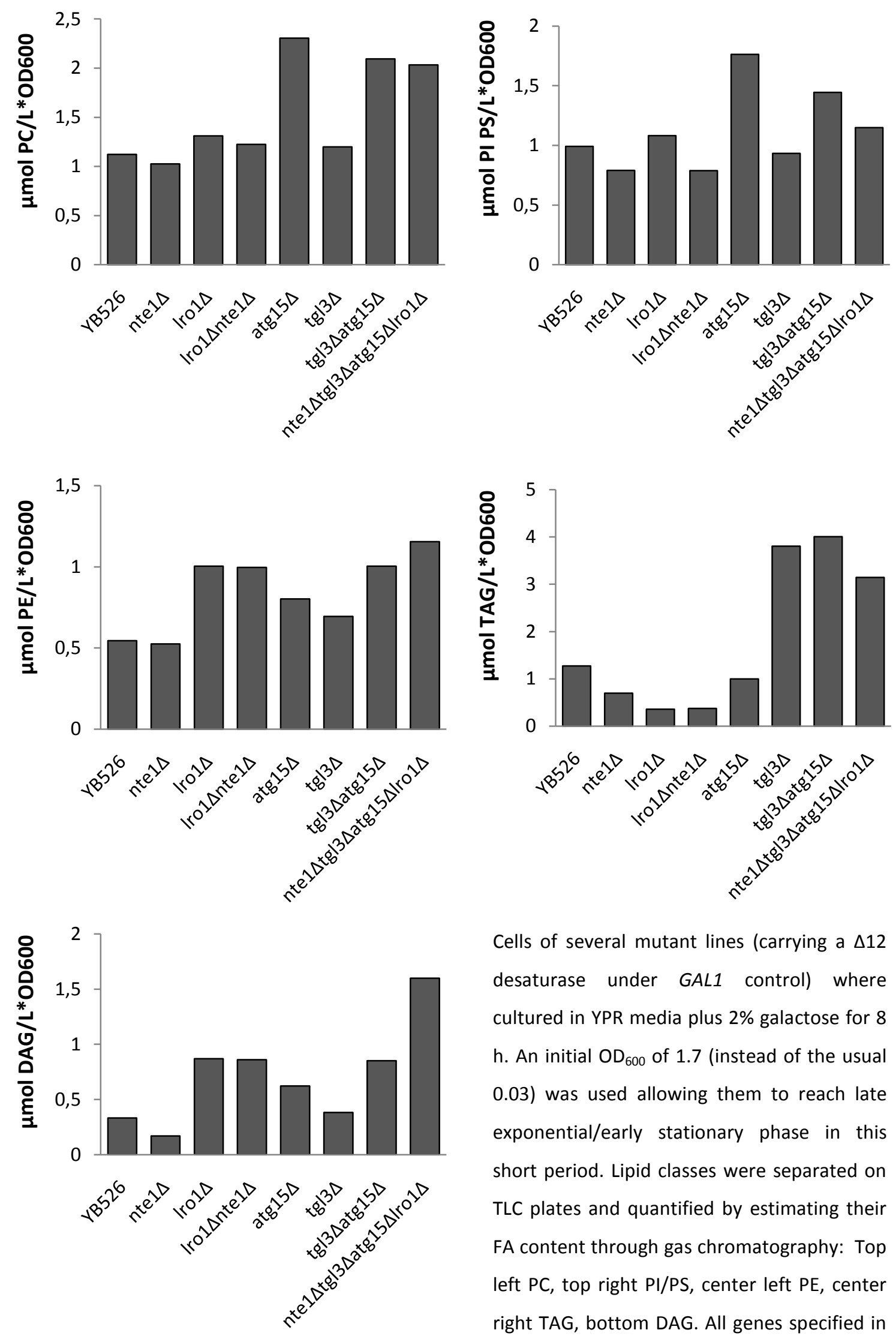

Cells of several mutant lines (carrying a $\Delta 12$ desaturase under GAL1 control) where cultured in YPR media plus 2\% galactose for 8 h. An initial $O D_{600}$ of 1.7 (instead of the usual 0.03) was used allowing them to reach late exponential/early stationary phase in this short period. Lipid classes were separated on TLC plates and quantified by estimating their FA content through gas chromatography: Top left PC, top right $\mathrm{PI} / \mathrm{PS}$, center left $\mathrm{PE}$, center right TAG, bottom DAG. All genes specified in the figure labels correspond to deletions on

Figure 30. Quantification of various lipid classes. YB526 background. 
Cells carrying an NTE1 deletion display a small decrease in the content of PI/PS, TAG and DAG and are unaffected in their PC and PE content. Cells deficient for $L R O 1$ display the expected reduction in TAG content and a remarkable increase in their PE and DAG content. Notice that the increase in these two lipid classes can also be observed upon

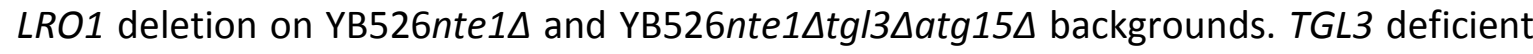
cells result in the expected increase of TAG content and an otherwise unaffected class distribution. Deletion of ATG15 leads to a higher content of all PL classes determined as well as to a higher DAG content. The amount of TAG might appear to be reduced in these cells but the difference is too small to provide certainty without a larger sample

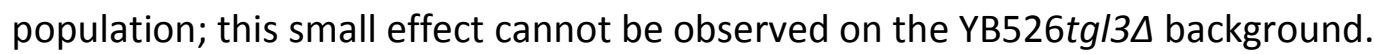

While these results are undoubtedly useful, it must be kept in mind that the experimental conditions behind figure 30 are not identical to those behind figures 11 to 24 and 29.

\subsection{Assessment of cell viability}

Although the optical density of a culture is a good indication of the cells growth, an additional assay was performed on a subset of our mutant cell lines to judge whether or not, and to what extent, the numerous gene deletions introduced affect cell fitness. For this test cells were grown to late stationary phase (136 h) in YPR media. Cell concentration in the culture was determined by counting under a light microscope and aliquots of the cultures where plated on YPD - agar. The colonies appearing on the plates were counted and the ratio of successful colony formation to the number of cells plated was calculated. This ratio was the same for the wild type (YB332) and the ACS deficient strain (YB526). Deletions of ATG15, PEX3, LRO1 and the combined deletion of LRO1, $D G A 1, A R E 1, A R E 2$ and $A T G 1$ resulted in a larger fraction of cells retaining viability after stationary phase. On the other hand, deletions of TGL3, DGA1, ATG1 and the combined deletion of YEH1, YEH2, TGL1 and TGL3 reduced the fraction of viable cells. Some deletion combinations including genes with an opposite effect on the fraction of viable 
cells (Iro1 $\Delta d g a 1 \Delta$ and $\operatorname{tg} / 3 \Delta a \operatorname{tg} 15 \Delta$ ) resulted in a value intermediate to those of the corresponding single deletions.

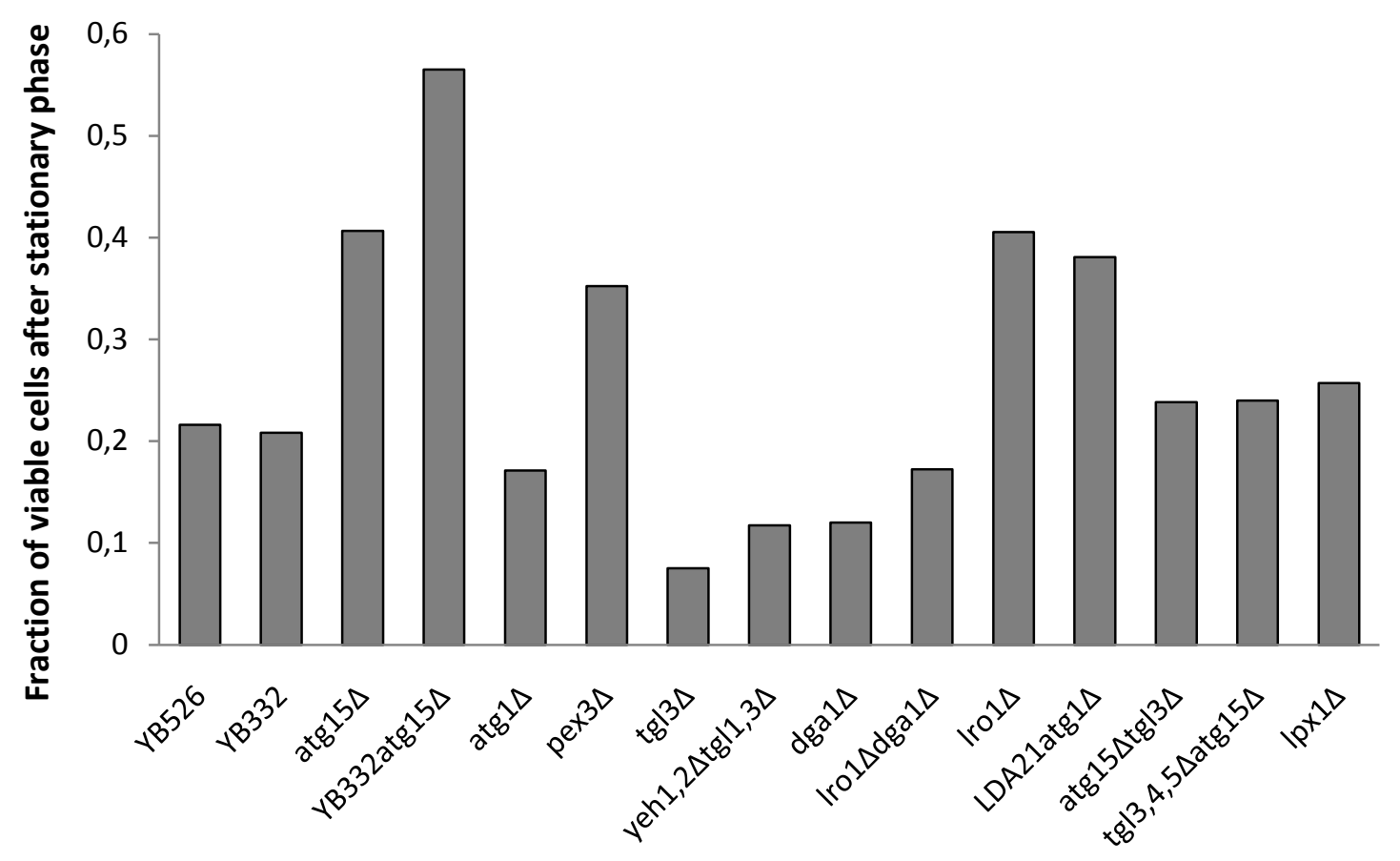

Figure 31. Degree of cell viability. The fraction of cells, out of the total present in an aliquot of a culture in stationary phase (136 h), that succeeded in forming colonies upon transfer to rich solid media, is an indication of the cells ability to survive a period in stationary phase and to resume active replication. Except where YB332 is indicated, all genes referred in the figure label correspond to deletions on the YB526

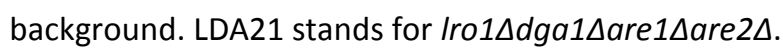

\subsection{LysoPE as a source of ethanolamine in PLB deficient cells}

Cells deficient for the phosphatidylserine decarboxylases Psd1p and Psd2p are ethanolamine auxotrophs. When the exogenous source of ethanolamine is provided in the form of lysoPE, the cells utilize it by converting lysoPE into PE by Ale1p mediated acylation. However, in absence of Ale1p the lysoPE is deacylated and further degraded to ethanolamine which is then used through the Kennedy pathway (Riekhof et al. 2007). This provided us with an opportunity to challenge two of our findings: first, that PLBs are not indispensable for PL deacylation and second, that Atg15p is involved in PL deacylation. For this purpose the genes coding for the phosphatidylserine decarboxylases 
Psd1p and Psd2p were deleted in the background of a mutant deficient for ALE1 and all known phospholipase B genes, resulting in the sevenfold mutant W303plb1 1 plb2 $2 p / b 3 \Delta n t e 1 \Delta a l e 1 \Delta p s d 1 \Delta p s d 2 \Delta$. Figure 32 demonstrates that psd1 1 psd $2 \Delta p / b 1 \Delta p / b 2 \Delta p / b 3 \Delta n t e 1 \Delta a l e 1 \Delta$ cells are viable with lysoPE as the only source of ethanolamine, while the additional deletion of ATG15 compromises their viability. This result supports our finding that Atg15p might be more important for the degradation of phospholipids than the Plb proteins and Nte1p altogether.

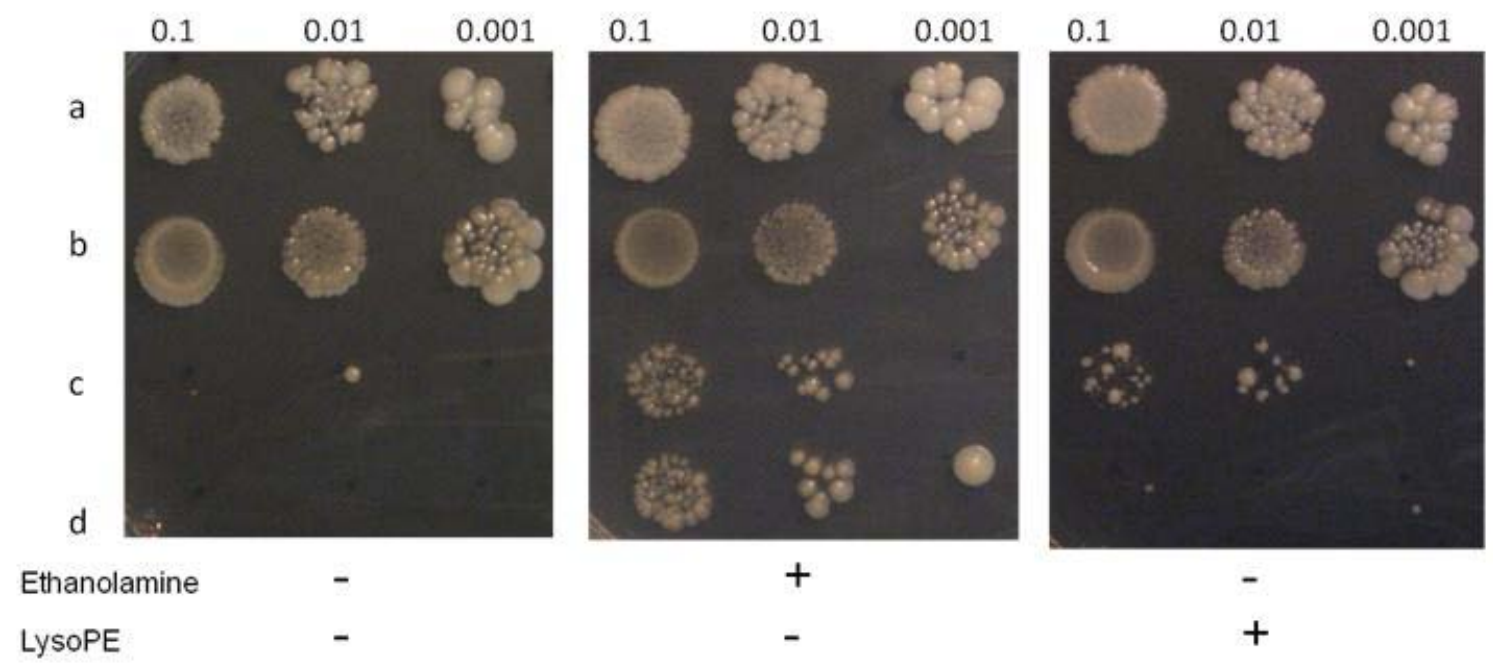

Figure 32. PLBs are not required for lysoPE deacylation.

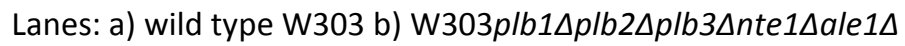

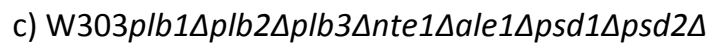

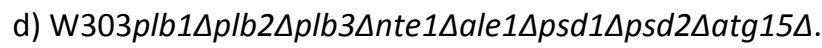

Cells where grown in YPD media for 24 hours, washed twice with water and diluted to the OD indicated above the columns. $5 \mu$ laliquots where plated on glucose SD media with no ethanolamine or lysoPE (left), $5 \mathrm{mM}$ ethanolamine (center) or $0.4 \mathrm{mM}$ lysoPE (right). The cells on lanes a and b are not auxotrophic for ethanolamine and survive in all conditions. The ethanolamine auxotrophic cells on lane c survive on lysoPE, indicating that the PLBs are not indispensable for lysoPE deacylation. Additional deletion of ATG15 in the cells on lane $d$ makes them unable to utilize lysoPE as a source of ethanolamine, indicating that the PLB deficient strain relies on Atg15p for lysoPE metabolization. 



\section{Discussion}

\subsection{Potential mechanisms for lipid deacylation and their impact on the equilibrium of different fatty acid pools}

\subsubsection{Free and bound fatty acid pools in acyl-CoA synthetase deficient cells}

The initial purpose of this work was the identification of the mechanisms responsible for lipid deacylation in S. cerevisiae leading to both PL degradation and PL remodeling; although the work often moved towards an analysis of FA homeostasis as a broader and more general phenomenon than deacylation alone, this initial purpose remained the central one at all times. As mentioned in the introduction, one of the main technical difficulties for the direct analysis of lipid deacylation in vivo as a specific individual process (and not indirectly as a component of lipid homeostasis in general) is the rapid metabolization of the molecules derived from it. In the case of fatty acids, metabolization is necessarily initiated by conversion of the free fatty acid into its activated form, acylCoA. Accordingly, the YB526 strain, in which the genes coding for the acyl-CoA synthetases Faa1p, Faa2p, Faa3p and Faa4p have been deleted, presents a strong FFA accumulating phenotype, providing an excellent model for the assessment of the role of diverse proteins and metabolic processes in lipid deacylation and FA homeostasis.

The effect of these deletions on the total content of free fatty acids is indeed dramatic (figure $11 \mathrm{~A}$ ), generating an 84 time increase from $0.83 \mu \mathrm{mol} / \mathrm{L}^{*} \mathrm{OD}_{600}$ in WT to $69.88 \mu \mathrm{mol} / \mathrm{L}^{*} \mathrm{OD}_{600}$ in the mutant. It is a well known fact that lipid turnover is a common process in cells and that it plays a major role in leading them to attain their steady state lipid composition. These results, however, highlight the actual magnitude of the phenomena: by late stationary phase, the cells have turned over an amount of FA corresponding to four times their total content of esterified FA. Given that in WT cells these FA are activated and can reenter lipid metabolism, it also becomes clear that lipid turnover and FA recycling make a major contribution to the acyl-CoA pool in addition to de novo FA synthesis. 
The content and profile of esterified FA is also affected in the mutant. Total esterified FA drops $20 \%$, from $21.9 \mu \mathrm{mol} / \mathrm{L}^{*} \mathrm{OD}_{600}$ in the wild type to $17.5 \mu \mathrm{mol} / \mathrm{L}^{*} \mathrm{OD} 600$ in the mutant (figure 11B). This decrease is driven by a major reduction in 16:1 and a minor one in 16:0, while the content of 18:0 and 18:1 actually show small increases. The ratio of 16- to 18carbon chains is thus strongly affected going from 1.66 in the wild type to 0.87 in the mutant, while the ratio of saturated to unsaturated FA remains almost unaffected $(0.24$ in the wild type, 0.29 in the mutant).

\subsubsection{Phospholipases B}

Initially, phospholipases B appeared as the most likely candidates to stand behind the lipid deacylation phenomenon revealed by the use of ACS deficient cells. As indicated in section 2.4., previous work established that the FFA pool which accumulates in these mutant cells is not only derived from lipid deacylation (as opposed to hydrolysis of acylCoA directly derived from de novo FA synthesis, a reaction the biological sense of which would be difficult to explain but which is at least chemically possible), but more specifically, that at some point prior to their release into the FFA pool the vast majority of FA might have in particular been part of a phospholipid, the deacylation of which is by definition the task of PLBs. Furthermore, throughout the available literature, almost every discussion of lipid deacylation in the context of PL remodeling mentions PLBs in one way or another. That the deacylation step in PL remodeling should be mediated by a phospholipase A- or B- like mechanism seems to be a matter of general agreement; however, when not just the mechanism but the actual enzymes need to be mentioned, most authors take good care of referring to Plb1p, Plb2p, Plb3p and Nte1p only as the known PLBs of yeast and therefore as plausible candidates without assertively postulating them as responsible for the deacylation step in PL remodeling. Two major reasons support this caution: First, a fairly obvious reason, their involvement in endogenous PL remodeling had not been experimentally verified. Second, these enzymes possess lysophospholipase in addition to phospholipase B activity (Fyrst et al. 1999; Lee et al. 1994; Merkel et al. 1999), meaning that, although they could produce the lysolipids that should mediate PL remodeling, they could also degrade them. 
To our knowledge, the present work constitutes the first direct evaluation of these proteins involvement in the process of remodeling the endogenous PL content of the cell. However, a recent report by Tanaka and coworkers (Tanaka et al. 2008) showed that deletion of $P L B 1, P L B 2$ and $P L B 3$ on a pem1 1 pem $2 \triangle$ background did not affect the ability of the choline auxotrophic pem $1 \Delta$ pem $2 \Delta$ strain to grow in media containing short acyl chain $\mathrm{PC}\left(\mathrm{diC}_{8} \mathrm{PC}\right)$ as choline source, despite strong evidence indicating that utilization of $\mathrm{diC}_{8} \mathrm{PC}$ proceeds at least partly through a remodeling mechanism. While their results seem too exempt Plb1p, Plb2p and Plb3p from involvement in the remodeling of an entirely unnatural exogenously provided PL species, our results extend this exemption to Nte1p and, most importantly, refer to the endogenous and naturally produced PL content of the cell.

The conflictive role of PLBs as mediators of PL remodeling due to their additional lysophospholipase activity made their testing all the more interesting and, in any case, it did not at all affect their candidacy as potential contributors to the FFA pool of YB526 cells since these FA are expected to derive not only from PL remodeling but also from PL degradation. Our results, however, surprisingly out rule their role in either process: Single deletion of the genes coding for any of the phospholipases B (Plb1p, Plb2p, Plb3p and Nte1p) in the YB526 strain had no effect on the FFA content of the cells. Simultaneous deletion of PLB1, PLB2 and PLB3 might appear to cause a small (10\%) reduction in the FFA content, however, the difference to the control strain is not significant (figure 12). This clearly indicates that none of these PLBs is indispensable for lipid turnover and in fact suggests that, at least under our experimental conditions, their contribution to basal phospholipid deacylation is negligible.

Since phospholipid remodeling is a constitutive process and must therefore be an essential component of basal deacylation, it now seems very unlikely that the PLBs could be involved in that particular process. While unquestionably important, this result is not really surprising, considering the proteins lysophospholipase activity. However, the lack of an effect on the FFA pool derived from the deletion of these lipases does not only speak against their involvement in PL remodeling, it speaks against their involvement in any sort of large scale constitutive lipid turnover process. In the case of Plb2p, which is most likely involved in handling extracellular lipids and lysolipids (Fyrst et al. 1999; 
Merkel et al. 1999), this result is not strange. Instead, for Plb1p, Plb3p and Nte1p whose role in the production of glycerophosphodiesters (GPD) from endogenous sources is well established (Patton-Vogt 2007), it is indeed surprising. One explanation would of course be that the additional mechanisms for lipid degradation are capable of masking the absence of the PLB enzymes. Alternatively, it might be accepted that these lipases could mainly degrade excessive phospholipids arising from specific culture conditions rather than taking part in constitutive degradation. The activity of Nte1p is known to behave in such fashion, specifically responding to increased PC synthesis through the CDP-choline pathway upon choline supplementation or elevated temperature (Fernández-Murray and McMaster 2005; Fernández-Murray and McMaster 2007; Dowd et al. 2001). While an equivalent behavior has not been demonstrated for Plb1p or Plb3p, it must be kept in mind that the experiments revealing their role in GPD secretion relied on supplementation with Choline and Inositol throughout the entire assay (Merkel et al. 1999; Merkel et al. 2005a). Within our experiments Nte1p did show a role in PL deacylation upon deletion of either DGA1 or ALE1, what, as will be discussed in sections 5.1.3. and 5.1.4., appear to be situations of increased PL synthesis.

Having out ruled PLBs as mediators in the formation of the FFA pool in the mutant strain YB526, we proceeded to test four alternative pathways that could lead, although indirectly in the first two cases, to the release of FA from PL to the FFA pool (sections 5.1.3. to 5.1.6.).

\subsubsection{Neutral lipid metabolism in PL synthesis and remodeling}

Until not too long ago, neutral lipids were basically regarded as a means for efficient (highly concentrated) storage of energy and building blocks, interacting with the broader features of lipid metabolism only as a source of matter upon poor media conditions. The possibility that NL metabolism could hold a more intricate relationship with lipid metabolism in general and with other cellular processes was then only a hinted question (Wagner and Daum 2005). Following a very rapid development in the mechanistic understanding of NL metabolism in yeast during the first years of this decade (reviewed in (Daum et al. 2007; Czabany et al. 2007)), this view has, mainly over the past two years, 
changed dramatically (Wagner et al. 2009; Kurat et al. 2009; Petschnigg et al. 2009; Rajakumari et al. 2008). Nevertheless, already three years ago, we had sufficient reasons to search for a role of NL metabolism, particularly TAG metabolism, in the release of FA from phospholipids to the FFA pool.

First, as mentioned in the previous section, the deacylation step in PL remodeling is generally believed to follow a phospholipase A- or B- like mechanism. The phospholipid:DAG acyltransferase (PDAT) Lro1p is not only an acyltransferase converting DAG into TAG, it is also a phospholipase A forming a lysolipid by retrieving an acyl chain from the sn-2 position of PC or PE prior to its transfer onto DAG. While the activity of Lro1p could be mainly regulated with regard to its role as a TAG synthase, relegating the production of lysophospholipids (lysoPL) to the category of a side effect, it could alternatively or additionally be regulated by the requirement of PL deacylation itself and is therefore a valid candidate as mediator of PL remodeling.

Second, in the work immediately preceding the present one (Mora 2006), the detailed time dependant evolution of both the FFA pool and the esterified FA pool in cells of W303 background lacking the genes FAA1 and FAA4, showed a behavior compatible with the established time dependant behavior of TAG synthesis and mobilization. Of course a compatible behavior does not imply a correlation, but it did seem as sufficient reason to search for such a correlation.

Deletion of $\angle R O 1$ on the YB526 background did in fact lead to a considerable decrease of FFA content (28\%, figure 13A). Lro1p mediated PL deacylation occurs at the expense of DAG while TAG and a lysolipid, the intermediary of PL remodeling, are produced. Since Lro1p does not directly produce a FFA, the decrease in FFA content seen upon LRO1 deletion is most likely mediated by a TAG lipase. Four such enzymes, $\operatorname{Tg}|2 p, \operatorname{Tg}| 3 p, \operatorname{Tg} \mid 4 p$ and TgI5p (Ham et al. 2009; Athenstaedt and Daum 2003; Athenstaedt and Daum 2005), are present in yeast. Deletion of TGL3 does in fact cause approximately the same decrease in FFA content as the deletion of $L R O 1$ (23\%, figure 13A). Deletion of only TGL2,

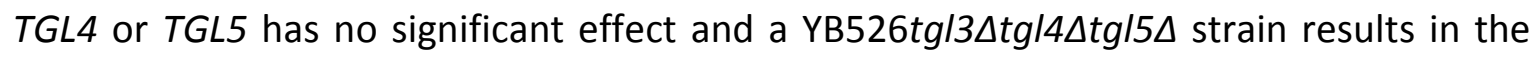
same FFA content as the YB526tgl3 $\Delta$ (figure 14A). 
In both the $\operatorname{tg} / 3 \Delta$ and Iro1 $\Delta$ mutants an increase in esterified fatty acids can be observed (figure 13B), corresponding to elevated levels of TAG in the YB526tg/3D strain and to increased levels of DAG and PE in the YB526/ro1 $1 \Delta$ strain where, on the other hand, a decrease of TAG was encountered (figures 29 and 30). However, analysis of further mutants reveals that the situation is more complex than a direct transfer of FA from PL to TAG to the acyl-CoA pool. First of all, the decreases in FFA independently observed upon deletion of only $L R O 1$ or TGL3 are not additive: the mutant YB526/ro1 $\Delta \operatorname{tg} / 3 \Delta$ contains the same amount of FFA as either of the strains with only LRO1 or TGL3 deleted (figure 15A). This result is certainly surprising given that Iro1 $1 \Delta$ cells are by no means devoid of TAG: Under standard culture conditions Dga1p is responsible for most TAG synthesis, closely followed by Lro1p (Sorger and Daum 2002; Oelkers et al. 2002) while Are1p and Are2p make a minor contribution (Sorger et al. 2004). Deletion of DGA1 causes a reduction in the content of esterified FA (figure 15B) and introduces no change in the size of the FFA pool (figure 15A), this behavior of the FFA pool is on its own a very unexpected result but we will first focus only on the interaction of DGA1 and TGL3 deletions. The reduction in the content of esterified FA caused by DGA1 deletion is not reverted in the YB526tgl3 $\Delta$ dga1 $\Delta$ mutant (figure 15B). This is true despite the fact that, as mentioned before, the YB526tgl3 $\Delta$ strain contains more esterified FFA than the YB526. Furthermore, paralleling the relation between the YB526/ro1 $\Delta$ tgl3 $\Delta$ and YB526/ro1 $\Delta$ strains, the FFA

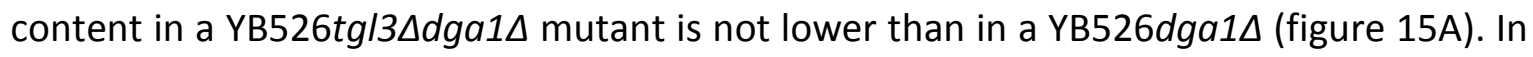
other words, while Tgl3p mediated TAG mobilization makes an important contribution to the FFA pool in the YB526 strain, Tgl3p appears to make no contribution to the FFA pool in either the YB526/ro1 1 or YB526dga1D strains, indicating that mobilization of the TAG produced by Dga1p could require the presence of Lro1p derived TAG and vice versa. Under these circumstances, the FFA decrease resulting from LRO1 deletion would not only be due to the absence of the Lro1p derived TAG pool that could directly act as substrate for Tgl3p, but also due to an inhibition in the mobilization of TAG produced by Dga1p. This interpretation appears to imply the existence of at least two metabolically (functionally) distinct pools of TAG, respectively derived from Lro1p and Dga1p activity. This is an extremely interesting possibility, but it is not necessarily true, and, in any case, is not an indispensable condition within our interpretation of the results. It is necessary to make a distinction between two quantitative pools and two truly distinct functional 
pools. In the first case, Lro1p and Dga1p each make a contribution to the total TAG content of the cell and we can refer to a pool derived from either protein in terms of mass balance, without necessarily implying that the actual molecules derived from one or the other pathway can be distinguished. In this case the apparently complete inhibition of Dga1p derived TAG mobilization by absence of Lro1p (and vice versa), would indicate that TAG mobilization is, to some extent, regulated by its rate of synthesis. Given that the overall rate of synthesis depends on two independent activities, each of which reflects the status of truly distinct source pools (the PL and acyl-CoA pools), synthesis could transduce an indication of the required rate of degradation. In the second case, an actual difference, either in spatiotemporal localization or in molecular composition should exist between TAG synthesized by one or the other acyltransferase. Furthermore, the cell should count with a mechanism that allows it to identify this difference. In this case, mobilization of one or the other pool would actually refer to degradation of molecules specifically produced by one or the other pathway, and the inhibition of the mobilization of one pool by absence of the other would not be necessarily related to the overall TAG content or rate of synthesis. Both cases are compatible with our results and our interpretation of them. The first one is clearly much simpler, particularly because understanding what characteristics could make TAG produced by one or the other pathway different, and how the cell could recognize those differences is not an easy task. Why, then, do we concern ourselves with the second case at all? Although our results do not require, and certainly do not prove, the existence of two functionally distinct TAG pools, they do suggest the possibility that such pools could exist. Although extensive research, well beyond the scope of this work, will be required before the matter can be resolved, the possibility alone is too relevant and interesting to let it pass unmentioned. In section 5.3., an experimental approach to evaluate the likelihood of functionally distinct TAG pools actually existing is outlined.

Simultaneous deletion of $\angle R O 1$ and DGA1 results in an esterified FA content that is not significantly different to the reference strain YB526 ( $p=55 \%$, figure 15B). While this result is indeed surprising, given that these cells have a strongly reduced TAG content, it is quantitatively in agreement with the independent increase and decrease of esterified FA seen in the YB526/ro1 1 and YB526dga1D strains respectively. The content of FFA in the 
YB526/ro1 $\Delta$ dga1D strain is the same as in the YB526dga1D (figure 15A), pointing once again to the scenario in which mobilization of TAG produced by one of the available pathways requires the presence of TAG produced through the alternative one, and in which the FFA reduction seen in the YB526/ro1 strain could, at least partly, correspond to an inhibition of Dga1p derived TAG mobilization. In a dga1 $\Delta$ background, mobilization of TAG synthesized by Lro1p has been inhibited; therefore, actual absence of that TAG pool by deletion of $L R O 1$ could not affect the FFA pool. Similarly, the inhibitory effect of LRO1 deletion on the mobilization of TAG produced by Dga1p would obviously be innocuous if there is no TAG produced by Dga1p. The equal content of FFA in the YB526/ro1 $\Delta$ dga1 $\Delta$ and YB526dga1 $\Delta$ strains is therefore in agreement with the unaffected

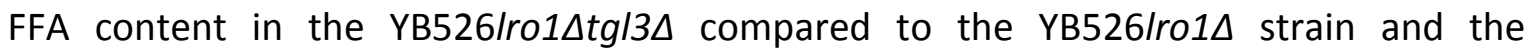

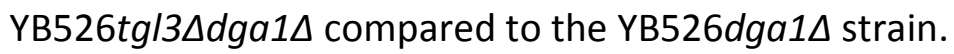

As indicated above, the fact that the size of the FFA pool in the YB526dga1 $\Delta$ strain is not different from the FFA pool in the reference strain YB526, was an unexpected result. It is indeed a surprising one given the strong effect of the $L R O 1$ or TGL3 deletions and even more as we propose that the effect of those two deletions is related to the mobilization of TAG synthesized by Dga1p. However, the unaffected FFA content in YB526dga1 $\Delta$ cells may propose a very interesting model: DGA1 deficient strains are faced with a pool of acyl-CoA that failed to be incorporated into TAG. If this led to either an accumulation of acyl-CoA or a down regulation of FA synthesis, a reduction in FFA content should result. Since that is not the case, it is apparent that the acyl chains in this acyl-CoA pool follow an alternative route still ending in the FFA pool. That route could be incorporation into either PL, STE or both, but causing a higher PL and/or STE turnover rate through a TAG independent mechanism rather than actual PL and/or STE accumulation. In line with the

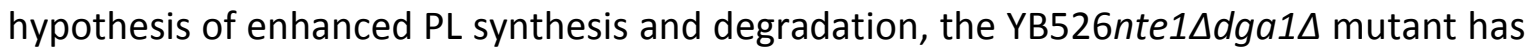
a lower FFA content than the YB526dga1D, despite the fact that the sole deletion of NTE1 did not affect the FFA content of the reference strain. This effect does not take place in a YB526nte1 1 Iro1 $\Delta$ mutant, which has as much FFA as the YB526/ro1 (figure 16A). This suggests that DGA1 deletion does in fact lead to an elevated rate of PL synthesis and degradation; measurements on a YB526dga1A strain additionally deficient for STE 
hydrolases would be required to establish whether it additionally leads to increased STE synthesis and mobilization, this however has not been done so far.

In a next set of experiments we investigated if STE metabolism also impacts the FFA pool. Simultaneous deletion of the genes coding for the STE synthases, ARE1 and ARE2, or of the genes coding for the STE hydrolases, TGL1, YEH1 and YEH2, on the YB526 background causes almost the same decrease in FFA content ( $32 \%$ and $25 \%$ respectively, figure $17 \mathrm{~A})$. This result suggests that STE mobilization does not exclusively occur at times of increased sterol requirement or upon interruption of de novo sterol synthesis, but rather that a continuous cycle of STE synthesis and mobilization takes place. This is in agreement with a recent report by Wagner and coworkers (Wagner et al. 2009) showing that the majority of sterol precursors make transit, as STE, through LP and are then recycled back into the biosynthetic pathway. However, in contrast to the above cited authors who reported an

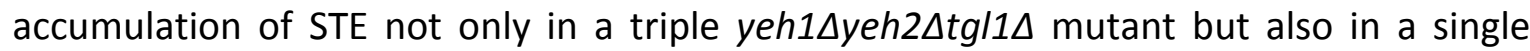
$\operatorname{tg} / 1 \Delta$ mutant, we only observed a strong FFA decrease in a YB526yeh1 $\Delta y e h 2 \Delta \operatorname{tg} / 1 \Delta$ strain

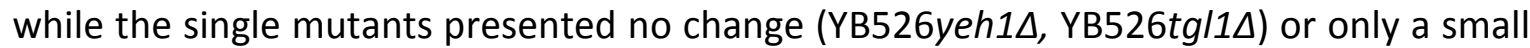
reduction (YB526yeh2A) (figure 17A).

Both STE synthesis and Dga1p mediated TAG synthesis utilize acyl-CoA. In the case of the YB526dga1 $\Delta$ strain no decrease in FFA content was observed and we interpreted this as an indication that the acyl-CoA is diverted towards PL synthesis but inducing a higher turnover rate of these species rather than an accumulation. In contrast, the YB526are1 $\triangle$ are2 $\Delta$ strain shows a direct FFA decrease; it is reasonable to assume that in this case the acyl-CoA that fails to be incorporated into STE is utilized by Dga1p and diverted towards TAG synthesis, however not accompanied by a higher TAG turnover rate but rather by TAG accumulation.

The results we obtained upon the simultaneous abrogation of TAG and STE synthesis in a YB526/ro1 $\Delta$ dga1 $\Delta$ are1 $\Delta$ are2 $\Delta$ strain support the idea that acyl-CoA not utilized for STE

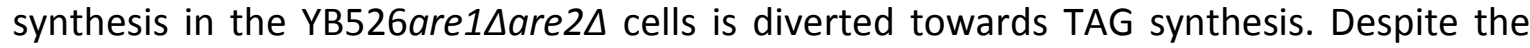
fact that neither the double deletion of $A R E 1$ and $A R E 2$ or of $L R O 1$ and DGA1 results in a reduced pool of esterified FA, the strain lacking all four genes contains a much lower amount of esterified fatty acids than the YB526are1 $\Delta$ are $2 \Delta$ or YB526lro1 $\Delta d g a 1 \Delta$ mutants (figure 18B). The FFA content of the YB526/ro1 $\Delta$ dga1 $\Delta$ are1 1 are2 $\Delta$ strain is higher than 
that of the YB526are1 $\Delta$ are2 $\Delta$, in fact, as high as that of the YB526/ro1 $\Delta$ dga1 $\Delta$ mutant (figure 18A): In cells capable of TAG synthesis acyl-CoA prevented from entering the STE synthesis and degradation cycle could enter a lipid accumulative pathway resulting in lower FFA, in cells devoid of TAG synthesis this acyl-CoA would enter a non accumulative pathway (PL synthesis and enhanced PL turnover) resulting in an ultimately unaffected FFA pool and lower esterified FA content. The diversion of acyl-CoA from STE synthesis into TAG synthesis upon deletion of ARE1 and ARE2 is in agreement with results presented by Sandager and coworkers (Sandager et al. 2002) indicating a reduction of STE accompanied by an increase of TAG content in an are $2 \Delta$ mutant with intact DGA1. However, another report by Zweytick and coworkers (Zweytick et al. 2000b) shows a decrease and a disappearance of STE in are $2 \Delta$ and are $1 \Delta$ are $2 \Delta$ cells respectively, without any concomitant change in TAG content. It must be noted that this two authors utilized different S. cerevisiae strains in their studies (Sandager: SCY62 congenic with W303; Zweytick: FY834).

A mutant in which we simultaneously interfere not with the synthesis but with the mobilization of both STE and TAG, YB526yeh1 $\Delta y e h 2 \Delta \operatorname{tg} / 1 \Delta \operatorname{tg} / 3 \Delta$, results in a $42 \%$ decrease of FFA content compared to the YB526 strain, a much stronger reduction than

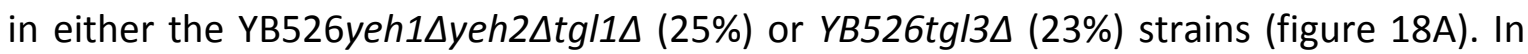
fact, the FFA decrease upon deletion of these four genes roughly corresponds to the addition of the decreases independently observed in the strains with only one of the pathways compromised.

Altogether these results present a picture in which NL metabolism can be deeply and centrally involved in PL species homeostasis. First of all, as shown by the

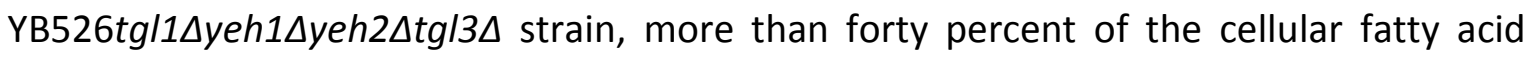
supply which is not directly derived from de novo synthesis is mediated by TAG and STE metabolism. Second, Lro1p mediates one quarter of the total FFA production. This prominent role of Lro1p in FA trafficking speaks strongly in favor of its candidacy as a major mediator of PL remodeling. It is clear that since in this case FA release should be additionally mediated by a TAG lipase, a one to one relation between PL deacylation and FFA production does not need to hold, which provides the cell with a unique opportunity to regulate the acyl species composition of the PL and acyl-CoA pools independently from 
each other. Third, the fact that we encounter what appears to be increased PL synthesis and degradation upon DGA1 deletion indicates that when Dga1p is present, it could serve to modify the magnitude and/or composition of the acyl-CoA pool in order to prevent the synthesis of unnecessary or inconvenient phospholipids. It could also promote the synthesis of $\mathrm{PL}$ with particular species composition by decreasing the relative concentration of competing fatty acid species in the acyl-CoA pool. And fourth, our results show that constitutive degradation of Lro1p produced TAG requires Dga1p mediated TAG synthesis and, conversely, continuous mobilization of TAG derived from Dga1p activity depends on the presence of a TAG pool produced by Lro1p. It is unlikely that this exact same behavior could be reproduced under different conditions, particularly under a situation of dire FA requirement; however, what lies underneath the specific behavior that we have observed, is an indication that the regulation of TAG mobilization is preceded by an integration of the activity of these two independent biosynthetic pathways. Under normal growth conditions and in presence of both Dga1p and Lro1p, this would allow the cell to integrate the effect of the acyl-CoA status on Dga1p activity with the effect of PL species composition status on Lro1p activity, and respond with TAG mobilization that is not exclusively tied, neither quantitatively nor qualitatively, to the amount or profile of TAG synthesized by either of the pathways alone. This holds true regardless of whether the activities of Lro1p and Dga1p actually lead to the formation of functionally distinct TAG pools or not. If they do, integration of the independent activities could be mediated by recognition of the specific pools, furthermore, regulation of TAG mobilization could in fact be cross regulation of the mobilization of each proteins product by the activity of the other one, providing enormous qualitative flexibility. If Lro1p and Dga1p do not produce truly distinct pools, the integration would probably depend only on the rates of synthesis.

These results make a clear and major contribution to the emerging perspective of NL metabolism being involved in cellular processes far beyond energy storage. Fatty acid transit through the NL pool might act as a means to allow rapid regulation of both the $\mathrm{PL}$ and the acyl-CoA pools size and acyl chain composition with a higher degree of independence from each other and from de novo FA synthesis, an ability that is clearly dispensable under ideal growth conditions but that might become critical under stress. 
The requirement of TAG mobilization upon transfer of cells in stationary phase to carbon rich media to allow rapid growth (Kurat et al. 2006) as well as the recently demonstrated cell cycle dependant regulation of fatty acid supply through TAG mobilization (Kurat et al. 2009) constitute cases in which NL mediation uncouples PL synthesis from de novo FA synthesis. On the other hand, the protective effect that the ability to synthesize TAG seems to provide to the cells against an excessive and unbalanced supply of fatty acids in the media (Lockshon et al. 2007; Petschnigg et al. 2009), as well as our observation that impaired NL synthesis appears to increase the flow of endogenous FA into PL and augment PL degradation, indicate a role of NL synthesis in avoiding a direct reflection of acyl-CoA availability, derived either from FA import or from FA synthesis, on PL synthesis. Our results further suggest a role of NL metabolism in uncoupling the content of the acylCoA pool from PL remodeling: PL deacylation through Lro1p instead of a PLB enzyme prevents its direct incidence on the acyl-CoA pool, however, an effect of Lro1pdependent TAG synthesis on the regulation of TAG mobilization would allow for a controlled effect of PL remodeling on the acyl-CoA pool without tying the profile of $\mathrm{PL}$ deacylation to the profile of changes in the acyl-CoA pool.

\subsubsection{Phospholipases C, D, and lysophospholipid acyltransferases}

As mentioned at the end of section 5.1.2., once the role of PLBs in the production of FFA was refuted, and before we identified NL metabolism as well as some additional lipases discussed in the next sections (5.1.5. and 5.1.6.) as actual contributors to the phenomena, an additional, hypothetical, pathway indirectly leading to the release of FA from PL was considered. This pathway constitutes a reversal of PL synthesis.

During PL synthesis, G3P/DHAP and LPA acyltransferases utilize acyl-CoA for the production of LPA and PA respectively. PA is then directly utilized for PL synthesis or converted by a PA phosphatase to DAG, which can then be turned into PL. It is a commonly known fact that the last part of this pathway can be reverted: phospholipids can be partially degraded without actual deacylation through the action of phospholipases D and C, respectively leading to the production of PA and DAG (which can then be converted to PA by action of a DAG kinase). What is not so commonly recognized 
is that the initial part of the pathway can also be reverted and might in fact be reversible. It has been demonstrated, in some mammalian models, that the same acyltransferases responsible for the acylation of the glycerol backbone during PA synthesis are also capable of deacylating it, leading to the release of acyl-CoA (Yamashita et al. 2001; Yamashita et al. 2003; Jones and Hajra 1983). Whether the acyltransferases available in yeast display the same behavior has not yet been fully demonstrated, however it appears to be the case (S. Stymne, personal communication).

Although this pathway would release acyl-CoA rather than FFA, the enzymatic hydrolysis of acyl-CoA is viable. In mammalian cells, the thioesterases required for this are known to be present in most cellular compartments (Faergeman and Knudsen 1997); in yeast, however, only one thioesterase (Tes1p) localized to the peroxisome has been identified (Jones et al. 1999).

It must be admitted that a flow of matter through this pathway in a magnitude comparable to that of the FFA accumulation in YB526 cells is extremely unlikely. However, given that at least the theoretical possibility of the pathway exists, it was considered worthy of experimental verification. Furthermore, partial degradation of phospholipids by a PLC or PLD, could be followed not only by deacylation through a reverse acting transferase, but also through a proper lipase. Although no enzyme with PA lipase activity has been identified in yeast, DAG can be used as a substrate for deacylation by the TAG lipase Tgl3p (Kurat et al. 2006) and probably by Tgl2p as well (van Heusden et al. 1998).

In order to test this hypothesis the FFA content of YB526 cells additionally deficient for the genes coding either the phospholipases C or D (PLC1 and SPO14), or the LPLATS ALE1, or TAZ1 were quantified. None of these disruptions led to a decrease in the FFA content relative to the reference strain. While interference with Plc1p activity had no effect on the FFA content (section 4.3.2.), deletion of either SPO14, ALE1 or TAZ1 actually derived in a higher FFA content than that of the reference strain YB526 (figure 19A). Similarly, deletion of the LPAAT/LPLAT SLC1 on ACS deficient cells results in a FFA content above that of the corresponding background strain (M. Scharnewski, personal communication). This out rules the possibility that the activity of Plc1p or Spo14p, followed by the activity of $\mathrm{Tg}|3 p, \operatorname{Tg}| 2 p$, or other yet unidentified DAG or PA lipase, could constitute a prominent 
means of PL degradation. It also refutes the hypothesis that the reverse activity of a lysolipid acyltransferase followed by a thioesterase could make a significant contribution to the FFA pool of ACS deficient cells. In this last case, however, our approach only speaks against this pathway being involved in FFA production but, since it does not address the option of acyl-CoA produced by these mechanisms being utilized for lipid synthesis or $\beta$ oxidation, it does not necessarily out rule deacylation. It should be mentioned that neither Pgc1p, a recently identified protein with PLC activity specific for PG (Simocková et al. 2008), nor Fmp30p, a homolog of the human $\mathrm{N}$-acylphosphatidylethanolamine (NAPE)-specific phospholipase D (Merkel et al. 2005b), have been tested within our study.

As mentioned above, deletion of the LPLATs or of SPO14 did not only fail to reduce the FFA content of the cells but in fact lead to an increase. In the case of YB526ale1 $\Delta$ cells this increase was substantial, corresponding to $40 \%$ of the FFA content in the reference strain YB526. It was mentioned in the introduction that both Ale1p and SIc1p contribute to LPA acylation during PL synthesis as well as to the reacylation of other lysophosphodiesters during PL remodeling. Although it is clear that these two proteins have different substrate preferences regarding the acyl chain to be utilized, either of them can sustain growth alone and give rise to cells with almost unaltered lipid profiles (Benghezal et al. 2007). Our measurements coincide with this: the esterified FA profiles of the YB526 and YB526ale1A cells are almost identical (table 2). Even though these transferases have different substrate preferences they do not entirely exclude their unfavored substrate; therefore, an excess of relatively unspecific PL synthesis followed by selective degradation would constitute a viable mechanism to achieve an unaltered profile and would be in perfect agreement with the enhanced FFA production observed in YB526 cells upon ALE1 deletion. Recall from sections 5.1.2. and 5.1.3. that, even though the PLBs did not show a contribution to constitutive PL degradation in the YB526 strain, deletion of NTE1 did lead to a reduced FFA content in a YB526dga1 1 background, where we propose that due to an imbalance in the acyl-CoA pool the rates of PL synthesis

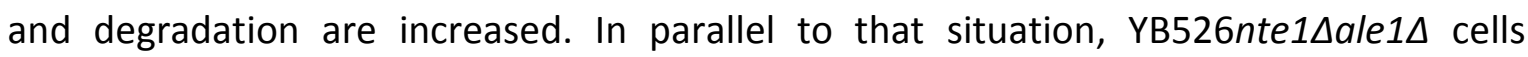
contain less FFA than YB526ale1A (figure 19A). Although lower than in the YB526ale1A,

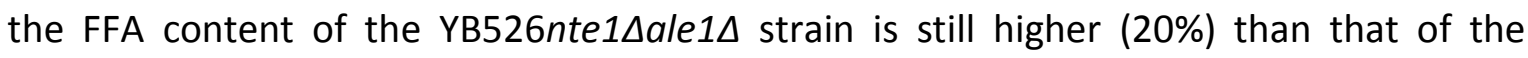


YB526, in the same way that the YB526nte1 1 dga1D contains less FFA than the

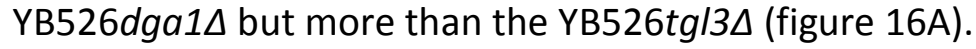

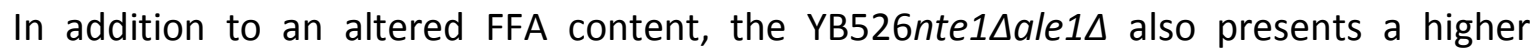
content of esterified FA than the reference strain (37\%, figure 19B), which is in agreement with the idea that the inability to regulate FA profiles during PL synthesis is compensated by an increased synthesis followed by degradation in which Nte1p would take part. Furthermore, in contrast to the YB526ale1 $\Delta$, the YB526nte1 $\Delta$ ale1 $\Delta$ strain does show a slightly altered esterified FA profile (table 2).

\subsubsection{Autophagy and PL homeostasis}

\subsubsection{Autophagy mediated PL deacylation}

The results discussed in section 5.1.3. demonstrate that NL mobilization makes a prominent contribution to FFA production and, as discussed there, link NL metabolism to $\mathrm{PL}$ species homeostasis both as a mediator of PL deacylation and through its impact on the acyl-CoA pool. In particular, the result obtained for the YB526yeh1 $\Delta$ yeh2 $\Delta \operatorname{tg} / 1 \Delta \operatorname{tg} / 3 \Delta$ strain indicates that over $40 \%$ of FFA production is directly derived from NL degradation. However, this obviously implies that the remaining $60 \%$ must follow a NL independent pathway and calls for the search of additional lipases involved in the process of constitutive deacylation. Under this premise the impact of the deletion of two putative lipases on the FFA content of YB526 cells was tested (Atg15p, this section; Lpx1p, section 5.1.6.).

The first of these genes to be deleted in our system was, thanks to a suggestion by Professor M. Thumm, the one coding for the vacuolar protein Atg15p. This protein was originally postulated to be a lipase based on the presence of a lipase motif identified by sequence modeling; furthermore a point mutation on the putative active site within this motif leads to loss of function. Cells deficient for ATG15 are defective in the breakdown of both Cvt and autophagic bodies leading to the accumulation of these vesicles within the vacuole (Teter et al. 2001; Epple et al. 2001). It is therefore believed that the lipolytic activity of Atg15p is required to render the vesicles open. Within our system Atg15p 
turned out to be the most prominent single player so far identified. Its deletion on the YB526 background leads to a decrease of 40\% in FFA content (figure 20A).

This result has large implications. Autophagy is well established as an important mechanism for protein degradation as well as for the removal of damaged or unneeded organelles. Its fundamental role in amino acid recycling is clear, in contrast its role in lipid turnover has not been frontally addressed ${ }^{3}$. The early stages of autophagy involve large scale membrane rearrangements both through the generation of the autophagosomal vesicles and their transit from the cytosol to the vacuole and through the withdrawal of entire organelles or portions of organelles from the cytosolic space. But, what happens with these large amounts of lipids at the later (degradative and regenerative) stages of autophagy? Macroautophagy (as well as the morphologically equivalent biosynthetic Cvt pathway) relies on double membrane vesicles. The outer membrane fuses with the vacuolar membrane while the inner one is released into the vacuolar lumen as an autophagic body (or Cvt body in the Cvt pathway). Since the vacuolar membrane cannot be allowed to simply grow unlimitedly, a mechanism must be in place to retrieve the fused membrane (or an equivalent portion) and maintain equilibrium. Retrograde vesicle traffic from the vacuole does not at all appear to take place in a sufficiently large scale. In fact, a single minor case of membrane efflux from the vacuole has been identified in $S$. cerevisiae (Bryant et al. 1998). In contrast to macroautophagy, microautophagy takes place through an invagination of the vacuolar membrane around the portion of cytosol to be engulfed, leading to a scission of the protruding membrane section and its direct release into the lumen as an autophagic body, partly compensating for membrane enlargement. However there is no reason to expect that macro- and microautophagy will necessarily occur at the same rate, therefore calling for further compensatory mechanisms. Tubular invaginations of protein-depleted vacuolar membrane portions have been observed to form and bud into the lumen; this process is in many ways equivalent to microautophagy and could constitute the means to maintain the equilibrium of the vacuolar membrane (Müller et al. 2000). Altogether, the actual mechanism of vacuolar membrane homeostasis and the final fate of membranes that

\footnotetext{
${ }^{3}$ A recent report by Singh and coworkers (Singh et al. 2009) links autophagy with TAG mobilization in mammalian cells, the relation of their results and ours is considered further ahead in section 5.1.5.2.
} 
fuse with it are currently recognized as unestablished, but appear likely to proceed largely through the budding of vesicles into the vacuolar lumen (see for example (Yorimitsu and Klionsky 2007a)).

In such case the question as to what happens with the lipids in membranes that fuse with the vacuole is probably the same as to what happens with the lipids in the membranes of the autophagic bodies, the Cvt bodies and the autophagocitized organelles. Given that the vacuole is a degradative organelle and the material transported to it is meant to be removed from the cell, it is natural to assume that the membranes entering the vacuolar lumen are disintegrated, the lipids composing them are degraded and the resulting molecules are exported to be recycled through lipid synthesis, or further degraded through $\beta$-oxidation, in an equivalent way to the well established amino acid recycling after vacuolar protein degradation. This however is not a trivial matter. It is essential for cell survival that the lytic mechanisms make an unequivocal distinction between the vacuolar membrane and the membranes in the lumen. This can be accomplished either by establishing a chemical protection of the vacuolar membrane, by restricting access of the hydrolases in an active state exclusively to the target membranes, by imposing on the hydrolases a substrate requirement matched only by the luminal vesicles, or by a combination of the above. As mentioned before, it is clear that the activity of the putative lipase Atg15p is required to destabilize the vesicles' membrane and make their content accessible to proteolytic degradation; this, however, does not require (neither does it rule out) the complete degradation of the membrane. Minor degradation by a very specific hydrolase at defined and restricted points could suffice to break its topological continuity and make the content of the membrane and the vesicle accessible to further hydrolases and would simplify the task of protecting the vacuolar membrane. Epple and coworkers (Epple et al. 2003) suggested that Atg15p could function in such fashion and predicted that, if in fact Atg15p displays such specificity, identifying its substrate would represent a major challenge; to date, almost nine years after the recognition of Atg15p as a putative lipase, its substrate has not been encountered. This model, however, poses a major problem: no other lipase-like protein appears to be present in the vacuole, meaning that instead of lipid subcomponents soluble in the 
aqueous phase the vacuole would need to secrete insoluble lipids, but, as mentioned above, vesicle efflux from the vacuole is rare.

The fact that ATG15 deletion brings down the FFA content of the YB526 strain by such an enormous amount as $40 \%$, demonstrates that massive amounts of lipids undergo degradation due to vacuolar transit. It is very unlikely, although of course not impossible, that an efflux of insoluble matter from the vacuole in such magnitude could have gone unnoticed. Furthermore if lipids (rather than FA, lysolipids and GPD) were to be secreted from the vacuole, they could be incorporated into membranes without necessarily undergoing degradation. Our result, therefore, points towards lipid deacylation occurring inside the vacuole in a large scale and, unless further unidentified lipases do reside in the vacuole, suggests Atg15p should be a rather unspecific lipase. It is difficult to understand how an unspecific lipase could show such reluctance to reveal its substrates through in vitro experiments. Although this could partly be a consequence of the unique conditions present in the vacuolar lumen or of the requirement for an unidentified cofactor (a possibility considered, although with a negative conclusion, by Epple and coworkers (2003)), it undeniably poses a major challenge to our hypothesis.

Beyond the problem of Atg15p specificity, our results with the YB526atg15 $\Delta$ strain (as well as those with further autophagy compromised strains discussed further ahead) constitute the first direct demonstration of the long assumed lipid degradation through vacuolar processes and indicate that autophagy plays a central role in phospholipid homeostasis and makes a major contribution to the acyl-CoA pool in wild type cells.

In agreement with the reported accumulation of intravacuolar vesicles and with the observed reduction in FFA content, the YB526atg15 $\Delta$ has a higher content of esterified FA than the YB526 strain (35\%, figure 20B). Most remarkably, the profile of esterified FA is strongly altered in this strain. The accumulation of esterified FA is mainly an accumulation of 16:0 followed by a large accumulation of 16:1, 18:0 increases slightly while 18:1 is almost unaltered. In consequence the relative content of esterified FA species in this strain results in a much higher proportion of 16:0 and 16:1, slightly reduced 18:0 and strongly reduced 18:1 (table 2). The ratio of 16- to 18-carbon chains is shifted from 0.87 in YB526 to 1.35 in the ATG15 deletion strain, while the ratio of saturated to unsaturated FA is shifted from 0.29 to 0.36 . This could probably mean that 
the vesicles transported to the vacuole are enriched in 16 carbon fatty acids while particularly poor in 18:1, and could shed light on the problem of Atg15p substrate specificity. The lipase could either display a preference for substrates with 16 carbon acyl chains, or, a really interesting possibility, it could require to be embedded in 16 carbon enriched membranes in order to be active. Furthermore, this result indicates that the turnover of large amounts of lipids through vacuolar transit (demonstrated by the decrease in FFA content) is not a passive side effect derived from the requirement of membranes to encompass the portions of cytosol to be degraded, but rather a process with an active (and prominent) role in the regulation of PL species composition.

To further analyze the role of autophagy in lipid deacylation we produced ATG1 deficient cells on the YB526 background. Atg1p is a protein kinase required, both through its kinase activity and through kinase independent properties, for the early stages of macroautophagy and the Cvt pathway (Matsuura et al. 1997; Abeliovich et al. 2003; Cheong et al. 2008; Straub et al. 1997). In contrast to the ATG15 deficient strain, where autophagic withdrawal of material from the cytosol proceeds normally and only the degradation of the vesicles and their content already inside the vacuole is compromised, an atg1ऽ strain is unable to form autophagosomes or Cvt vesicles preventing not only degradation but also sequestration of cytosolic material and membrane transit to the vacuole.

As in the previous case, deletion of ATG1 derived in a strongly reduced FFA content (33\% figure 20A) and an increased esterified FA content (32\% figure 20B). The profile of esterified FA in this strain is also modified compared to the YB526 strain (table 2), although to a lesser extent than in the YB526atg15 (16/18: 1.03; sat/unsat: 0.35). This strongly supports the idea that lipid degradation through vacuolar transit is not just a side effect but a highly relevant mechanism for the regulation of the cellular PL content.

It is fairly clear that in the case of ATG15 deletion the increase in esterified FA should mainly correspond to the accumulation of vesicles (and their content) inside the vacuole. In contrast, in the case of YB526atg1 cells the location of the excess esterified FA makes a very interesting question. They could perhaps be partly accounted for by an enlargement of the PAS (this is an entirely speculative statement probably difficult to verify given the poorly understood nature of the PAS) as well as by the permanence in 
the cytosol of organelles (peroxisomes, mitochondria) otherwise meant for degradation. It is not too stretched however, to expect an effect of ATG1 deletion, even if only transient, on the ER. First, the ER is a direct target of autophagy; second, it is the initial site of PL synthesis and therefore stands at the base of all non endocytic vesicle traffic and third, given that autophagy appears to be involved in the regulation of PL species composition it should directly interact with the ER which is central in this process (the LPAATs Ale1p and SIc1p as well as the PDAT Lro1p are localized in the ER, furthermore the ER is the origin of LP and the site of FA elongation and desaturation). Whether a potential effect of autophagy in lipid homeostasis directly at the ER should be a consequence of the ER being a target of autophagy or of it being the ultimate (most likely indirect) source of membranes for vesicle formation is of course not clear at this stage. However, even if it appears reasonable in light of our results to expect that blocking the early stages of autophagy could disturb lipid homeostasis at the ER, it seems even more reasonable to expect that the cell would attempt to reestablish ER homeostasis in order to prevent the complex biosynthetic machinery of this organelle from being compromised.

\subsubsection{Interaction of autophagy and NL metabolism}

As discussed in section 5.1.3., NL metabolism was shown to be a key player in the regulation of PL species composition; we therefore hypothesized that TAG metabolism could act as a compensatory pathway to avoid or minimize a disturbance of PL composition in the ER due to ATG1 absence. In fact, deletion of ATG1 leads to an increased TAG content both in YB526 and in YB526tg/3 $\Delta$ cells (figure 29). Most strikingly, the FA species composition of TAG accumulating in these mutants presents an increase in the relative content of $16: 0$ and $16: 1$ and a decrease in the relative content of $18: 0$ and 18:1 (table 3), resembling the alteration in total esterified FA profiles of the YB526atg15 strain. This is in very clear agreement with both the ideas that autophagy participates in the regulation of PL species by selectively utilizing or targeting membranes enriched in 16 carbon chains and that TAG synthesis serves as a mechanism to alleviate the imbalance in $\mathrm{PL}$ species composition resulting from the blockage of autophagy at an early stage. 
An alternative, in fact opposite, hypothesis was very recently presented by Singh and coworkers (Singh et al. 2009). They report, for mammals, that cells compromised in autophagy present a higher content of TAG and conclude that autophagy is involved in the degradation of LP and particularly in the mobilization of TAG. It could therefore be argued that the increase in TAG we observe upon deletion of ATG1 is not a consequence of increased TAG synthesis as a compensatory mechanism for PL accumulation, but rather an effect of impaired TAG degradation. This contradiction requires careful consideration. As shown in figure 29, not only the YB526atg1D contains more TAG than

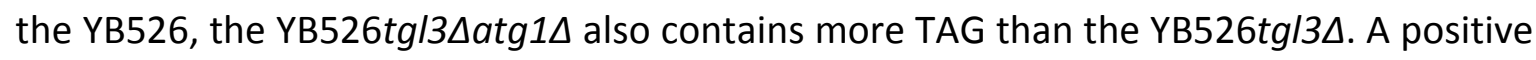
effect of ATG1 deletion on the TAG content of cells that are compromised in TAG degradation anyway, is more compatible with this effect being derived from a biosynthetic rather than a degradative process. Furthermore the YB526tg/3 $\Delta a \operatorname{tg} 1 \Delta$ strain presents a reduction in the content of FFA of 56\% compared to the YB526, a much greater reduction than the one encountered upon deletion of TGL3 or ATG1 alone (23\% and $33 \%$ respectively, figure $20 \mathrm{~A}$ ). This suggests that the contributions of TAG mobilization and vacuolar lipid degradation to the FFA pool are independent from each other. This is compatible with a scenario where Tgl3p mediated TAG mobilization is basically unaffected by the presence or absence of ATG1, while the blockage of autophagy at an early stage prevents the mobilization of large amounts of PL towards the vacuole and leads to the formation of a TAG pool that is not meant for degradation.

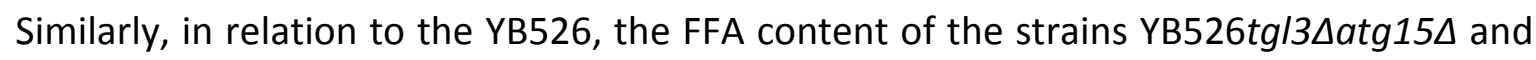
YB526Iro1 $\Delta$ atg15 decreases by 58 and $51 \%$ respectively, far above the effect of the

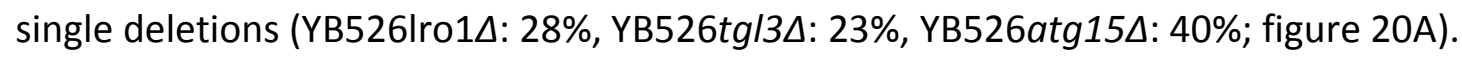

The additive nature of the effects indicates that the NL and autophagy pathways for FFA production are independent from each other and speaks against a role of autophagy in NL mobilization under our experimental conditions. This alone does not entirely rule out the possibility of such a role since independent pools of TAG could be differentially exposed to autophagic or regular Tgl3p mediated mobilization, however, the actual content of TAG in ATG15 deficient cells presents a much more radical objection to the hypothesis of Singh and coworkers. As shown in figure 29, YB526atg15 $\Delta$ cells do not only fail to present an increase in TAG content compared to YB526 cells but in fact have a 


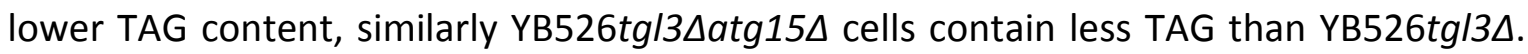
Reduced TAG levels upon deletion of ATG15 are not restricted to our ACS deficient background as YB332atg15 cells also contain less TAG than wild type YB332. If the higher amount of TAG in atg1 $\Delta$ cells were a consequence of impaired TAG mobilization due to interrupted transit of LP to the vacuole, as indicated by the work of Singh and coworkers, and not due to a higher TAG synthesis rate in response to altered PL composition, as derived from our results, the atg15 strains should necessarily also contain more TAG than their respective backgrounds, as LP would transit to the vacuole but would still fail to be degraded there.

We do not at all contest the actual results or observations of Singh and coworkers (2009), but we do consider that some of their interpretations could be reevaluated in view of our own results. It must, however, not be forgotten that their study and ours employ different organisms, and that, in consequence, the situation is not necessarily identical in both cases. In the cited work, autophagy is inhibited either through deletion or RNA interference of $A T G 5$ or ATG7 or through the addition of 3-methyladenine (3MA). Atg5p and Atg7p are required for the initial steps of autophagosome and Cvt vesicle formation (Kuma et al. 2002; Tanida et al. 1999; Kim et al. 1999b; George et al. 2000; Mizushima et al. 1998), 3MA is a kinase inhibitor that prevents autophagic sequestration (Blommaart et al. 1997). All this approaches can be considered equivalent to our deletion of ATG1 in that they block autophagy at its early stage and, accordingly, the actual result is the same in their case and ours: an increase in TAG content. We base our interpretation of this result as an increase in TAG synthesis on the observed alteration of FA species profile in these mutants and the knowledge that, first, the cell must try to maintain membrane composition and second, NL metabolism participates in the regulation of PL species composition. On the other hand we reject the interpretation of this result as an impairment of TAG mobilization based on the observed decrease rather than increase of TAG content in all ATG15 deficient strains tested. The results of Singh and coworkers do not include any mutant or experimental approach that is equivalent to our deletion of ATG15 in preventing vacuolar degradation while still allowing autophagic sequestration. They, however, do present further support to the idea of impaired TAG degradation beyond the increase in TAG content: measurements of the relative rate of $\beta$-oxidation. 
Given that TAG mobilization provides a large supply of fatty acids and can therefore induce $\beta$-oxidation, or alternatively that an elevated rate of $\beta$-oxidation implies a need for abundant FA and can therefore induce TAG mobilization, it is a generally accepted practice to assume a correlation between the rate of $\beta$-oxidation and the rate of TAG mobilization under specific culture conditions (Owen et al. 1979). Singh and coworkers observe a reduction in the rate of $\beta$-oxidation when autophagy is inhibited through the knockdown of $\operatorname{Atg} 5$ and therefore conclude that preventing autophagic sequestration compromises TAG mobilization. But there is a pitfall in this reasoning. Regardless of whether autophagy is specifically involved in TAG degradation or not, it is involved in the degradation of substantial amounts of $\mathrm{PL}$; therefore, independently of what happens with TAG, blocking autophagy causes a reduction in FA supply and should lead to a reduced relative rate of $\beta$-oxidation. In this particular regard however, it could be counter argued that the pitfall is in our reasoning: We disregard the rate of $\beta$-oxidation as a valid measurement of TAG mobilization under conditions of compromised autophagy by holding that the reduced rate of PL deacylation caused by such conditions would have the same effect. However, the present work is, to our knowledge, the first direct demonstration of large scale PL deacylation through autophagy and the main evidence of this deacylation is precisely the reduction of FFA content in the YB526 mutant background. It could therefore be argued that the reduction in FFA in our atg1 $\triangle$ strains is mainly derived from impaired TAG mobilization with only a minor contribution from PL degradation (although this would be in contradiction with our TAG quantification in $\operatorname{atg} 15 \Delta$ strains), in which case $\beta$-oxidation would still be a valid measurement of TAG mobilization. This dispute is entirely solved by our next mutant:

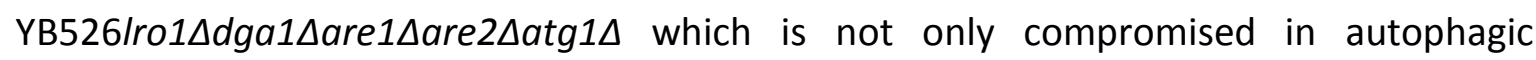
sequestration but is also unable to produce NL. Recall from section 5.1.3. that the FFA

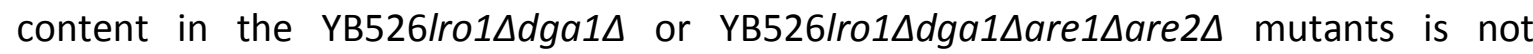
significantly different from the FFA content in the background strain YB526 (our interpretation of the complex interaction between the effects of Lro1p and Dga1p on the FFA pool was presented in section 5.1.3.). As indicated above, the YB526atg1 1 strain presents a $33 \%$ reduction in the content of FFA compared to the YB526. The YB526/ro1 $\Delta$ dga1 $\Delta$ are1 1 are2 $\Delta a \operatorname{tg} 1 \Delta$ mutant, on the other hand, presents a reduction of $31 \%$ (figure 21A). This data impressively demonstrates that the effect of blocking 
autophagic sequestration on FFA production does not differ in cells with or without NL, clearly indicating that the FFA reduction observed in YB526atg1D cells corresponds to impaired PL deacylation rather than to impaired TAG mobilization. It has to be admitted that the actual regulation of PL composition in these cells, impaired in what we have so far proposed as the two major pathways for this purpose, must be strongly deviated from standard behavior, but that is a matter we will come back onto further ahead.

The situation, of course, does not need to be black or white. Based on the evidence discussed so far, we are strongly convinced that under our experimental conditions autophagy makes no relevant contribution to TAG mobilization and instead TAG synthesis compensates for the lack of autophagic sequestration. We also suspect that in some of the cases where Singh and coworkers found higher amounts of TAG they might have been facing a situation of increased synthesis rather than impaired mobilization, but we do not believe this has to hold true under all conditions. We have no evidence at all indicating that TAG degradation through vacuolar transit is intrinsically unviable under all conditions and it must be considered that some of the experiments discussed in the work of Singh and coworkers were carried out under media conditions specifically meant to induce either NL accumulation or NL mobilization. Furthermore, they demonstrate the colocalization of LP and vacuole components and they present electron micrographs displaying LP within double membrane vesicles. In fact the sequestration of LP by vacuoles has also been observed in yeast.

It must also be admitted that our own hypothesis leaves at least two open questions we can currently not account for. First, it is difficult to explain why atg $15 \Delta$ cells actually contain less TAG rather than an unaffected amount. If we were to consider only the results obtained on the wild type background, it would be easy to propose that the reduced availability of FA due to impaired vacuolar PL degradation could lead to either a decrease in TAG synthesis or an increase in its mobilization. However, in our ACS deficient background where the cell is unable to utilize these FA anyway, such an explanation is not satisfactory. Second, the reduced TAG pools in the ATG15 deficient strains present a relative composition of FA species altered in a similar way than the increased TAG pool of the ATG1 deficient ones (i.e. an increased relative content of 16:0 and $16: 1$ and a reduced relative content of $18: 0$ and 18:1. Table 3 ), even though our 
model does not predict that the deletion of ATG15 should subject the ER membrane, or other membranes, to a similar stress as the deletion of ATG1.

As presented in section 5.1.3. and already reiterated several times through this work, one of our major findings is the prominent role that NL metabolism plays in the regulation of PL species composition. Now we have additionally established that autophagy constitutes a major player in PL deacylation and presented evidence strongly suggesting that the effect of this deacylation on PL homeostasis is not only quantitative but also has a qualitative effect on PL species composition. The way of action through which these two pathways affect the PL species composition is most likely of a very different nature. In the case of NL metabolism the effect can be thought of as occurring at a molecular level. For this purpose the FA composition of individual lipid molecules could be regulated either by deacylating them and thereby introducing them into the remodeling pathway or by enforcing (or discouraging) specific outcomes of de novo synthesis and lysoPL reacylation through a modulation of the FA species distribution in the available substrate. In the case of autophagy, on the other hand, the effect can be better thought of as occurring at the level of PL pools, rather than individual molecules, through the sequestration of membrane portions (or the use of membranes for vesicle formation) with particular FA species distribution. The additive effect of these pathways on FFA

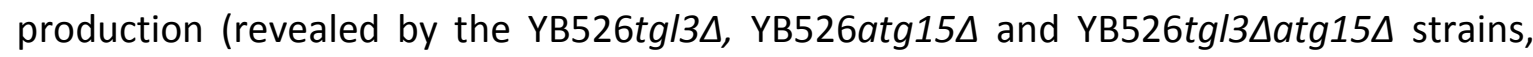
figure 20A) indicates that they are independent from each other; however, the changes in the TAG pool in the ATG1 deficient strains indicate that the NL pathway can react to compensate disturbances caused by a failure in the autophagy pathway. In this case the individual molecules in a general pool that has failed to be withdrawn by autophagy would be modified specifically. The pertinent question, then, is whether the opposite, or rather complementary, effect can also take place. That is, can the autophagy pathway react to counterbalance a perturbation derived from a compromised NL pathway by withdrawing a pool of lipids the composition of which has failed to be regulated through fine tuned de novo synthesis and PL remodeling? The results presented in figure $21 \mathrm{~A}$, show that while the YB526/ro1 1 dga1 $\Delta$ are $1 \Delta$ are2 $\Delta$ strain presents no change in its FFA content compared to the YB526 and the YB526atg15 presents a $40 \%$ decrease, the

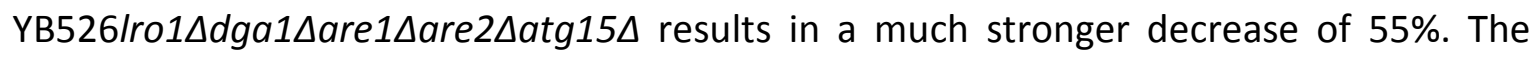


latter value is not significantly different to the decrease observed in the

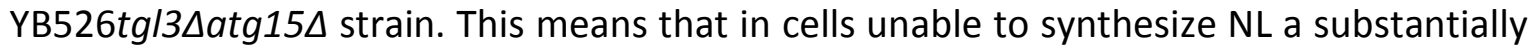
higher amount of PL are targeted for degradation in the vacuole and therefore indicates that the autophagy pathway does react, and in consequence might compensate, for the loss of PL species regulation through the NL pathway. This is a result of great significance. It is a well established fact that autophagy is induced in response to ER stress; the stress considered, however, relates directly to protein misfolding (see for example (Yorimitsu and Klionsky 2007b)). Our result, instead, points towards autophagic induction by stress derived from lipid imbalance.

Recall from section 5.1.3. that the deletion of NTE1 in absence of DGA1 resulted in a decrease in FFA content, indicating that direct deacylation of PL by a phospholipase becomes relevant once the PL pool has been altered by a situation of enhanced PL synthesis. However, as the decrease observed in FFA content was relatively small, it was also clear that additional mechanisms for PL deacylation come into action under such conditions. The stronger decrease of FFA concentration observed in the

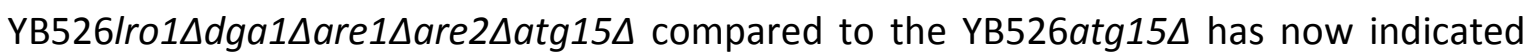
that autophagic sequestration is one of these mechanisms. In parallel to that, we have seen that at least one of the compensatory mechanisms for the disturbance caused by ATG1 deletion relies on a detour of matter into TAG synthesis. This puts the mutant strain YB526/ro1 $\Delta$ dga1 $\Delta$ are $1 \Delta$ are2 $\Delta a \operatorname{atg} 1 \Delta$ in a very complex situation. It is apparent that under these circumstances PL degradation through direct mechanisms (such as Nte1p mediated deacylation and perhaps other yet unidentified pathways) must be enhanced as the FFA content of these cells is not lower than that of the YB526atg1D strain (figure 21A). But it is also clear that compensation cannot be quantitatively complete given that the esterified FA content of these two strains is very similar (figure 21B),

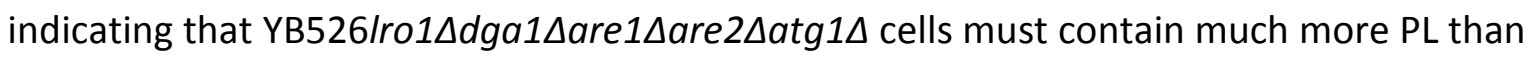
YB526atg1D cells which deposits substantial amounts of FA in form of TAG. The results presented in section 5.1.4., where a reduced ability to regulate PL species composition through de novo PL synthesis due to the absence of the LPAAT Ale1p is apparent, suggested that such challenge is matched trough enhanced unspecific synthesis and enhanced specific degradation in which Nte1p takes part. This would indicate that the 
activity of Nte1p can affect the PL pool qualitatively in addition to quantitatively and could be part of the reason for the viability and apparently unaltered fitness of YB526/ro1 $\Delta$ dga1 1 are $1 \Delta$ are $2 \Delta a t g 1 \Delta$ cells. A prominent question this opens is, if Nte1p activity and perhaps other mechanisms of a similar nature are so effective, why are they relegated to extreme situations and not involved in constitutive lipid remodeling under "normal" conditions? The answer to this could to some extent lay in the fully degradative nature the mechanism requires. As indicated in section 5.1.3. a major advantage of NL mediated PL remodeling is the uncoupling that it provides between the released acyl chains and the acyl-CoA pool. In this case the FA resulting from deacylation can be stored and utilized either for synthesis or energy production at a different time. In contrast, deacylation as provided by Nte1p has a direct impact on the acyl-CoA pool (in ACS competent cells) which would immediately affect PL synthesis or force the cell to degrade the acyl chains at a time that might not be ideal in terms of energy efficiency.

Before moving onto the figure that summarizes the findings so far presented, let us briefly put three further mutants into context, two of which constitute the strongest FFA

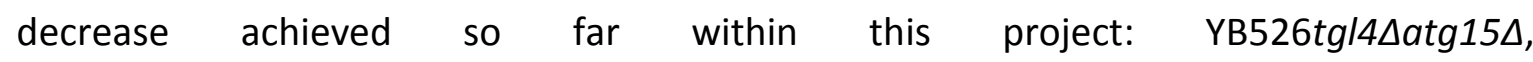

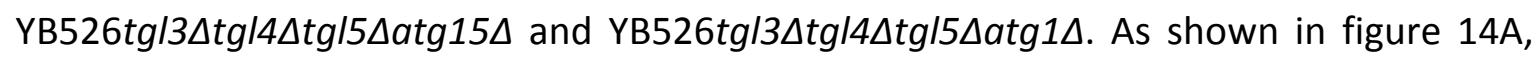
deletion of either TGL4 or TGL5 had no effect on the FFA content of YB526 cells, neither

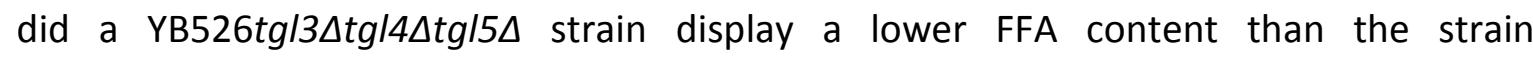

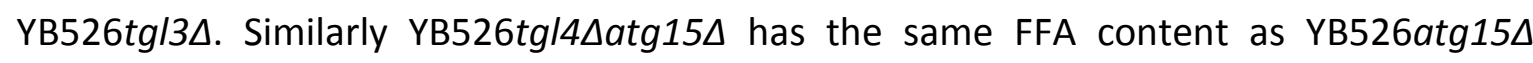

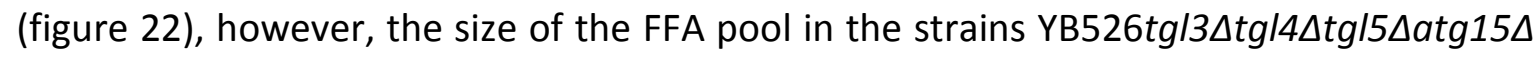

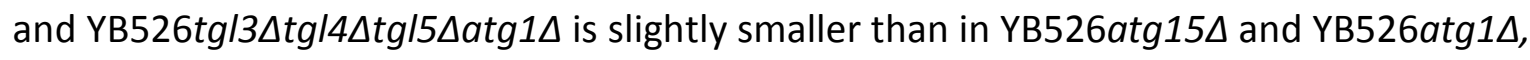
respectively. Whether this is due to enhanced activity of this two remaining TAG lipases as a consequence of the strongly reduced deacylating capacity of the $\operatorname{tg} / 3 \Delta$ plus autophagy deficient background, or whether the small contribution of Tgl4p and Tgl5p is present at all times but only becomes detectable once the size of the FFA pool has been sufficiently reduced making it a relatively larger contribution, we cannot tell at the moment. Given the small magnitude of the difference the effect is not of great relevance and we will not be further concerned with it, but it was considered meritorious of

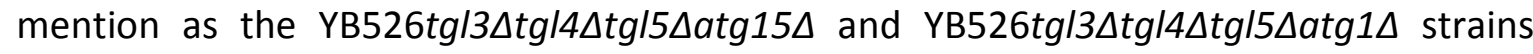
contain the smallest FFA pool within this work and, therefore, represent a landmark. In 
these two cases the FFA decrease is of 67 and $68 \%$, respectively. Considering that STE hydrolysis is still available in these strains and that the FFA content reductions in the

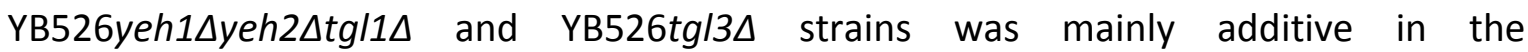

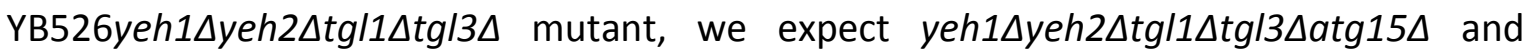
yeh1 $\Delta$ yeh2 $\Delta \operatorname{tg} / 1 \Delta \operatorname{tg} / 3 \Delta \operatorname{tg}|4 \Delta \operatorname{tg}| 5 \Delta a \operatorname{tg} 15 \Delta$ cells to result in a FFA pool reduction within 75 and $85 \%$. This, however, is still pending for experimental verification.

Figure 33 summarizes in a schematic form our interpretations of the results presented in this section and section 5.1.3. in terms of metabolite detours into alternative pathways.

Note that the boxes do not pretend to be on scale or represent relative sizes of different pools. The only exception to this is the pool of FFA directly derived from autophagy, which is larger than those derived from TAG or STE mobilization. The figures are balanced with the observed changes in FFA content. In the case of esterified FA the figures illustrate the detours of matter that we propose take place in specific mutants as well as the appearance or disappearance of transit and stationary pools or the conversion of a transit pool into a stationary one. This reflects observed changes in esterified FA content; however the figures are not strictly balanced for esterified FA since we do not know the relative size of the various pools. Note that the contribution of a transit pool to esterified FA quantification is uncertain and unrelated to its contribution upon turning into a steady pool.

The pathway labeled as mediated by Nte1p is likely to involve other yet unidentified elements. The model does not illustrate the observed reduction in TAG content in ATG15 deficient strains, the fact that DAG can be derived from PL in addition to PA, or the fact that an additional, so far unidentified, pathway for FFA production must exist. As

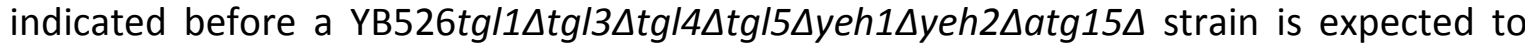
result in a FFA decrease of approximately 85\% compared to the YB526 strain. Therefore this unidentified pathway could be responsible for approximately $15 \%$ of FFA production in the YB526 strain. 

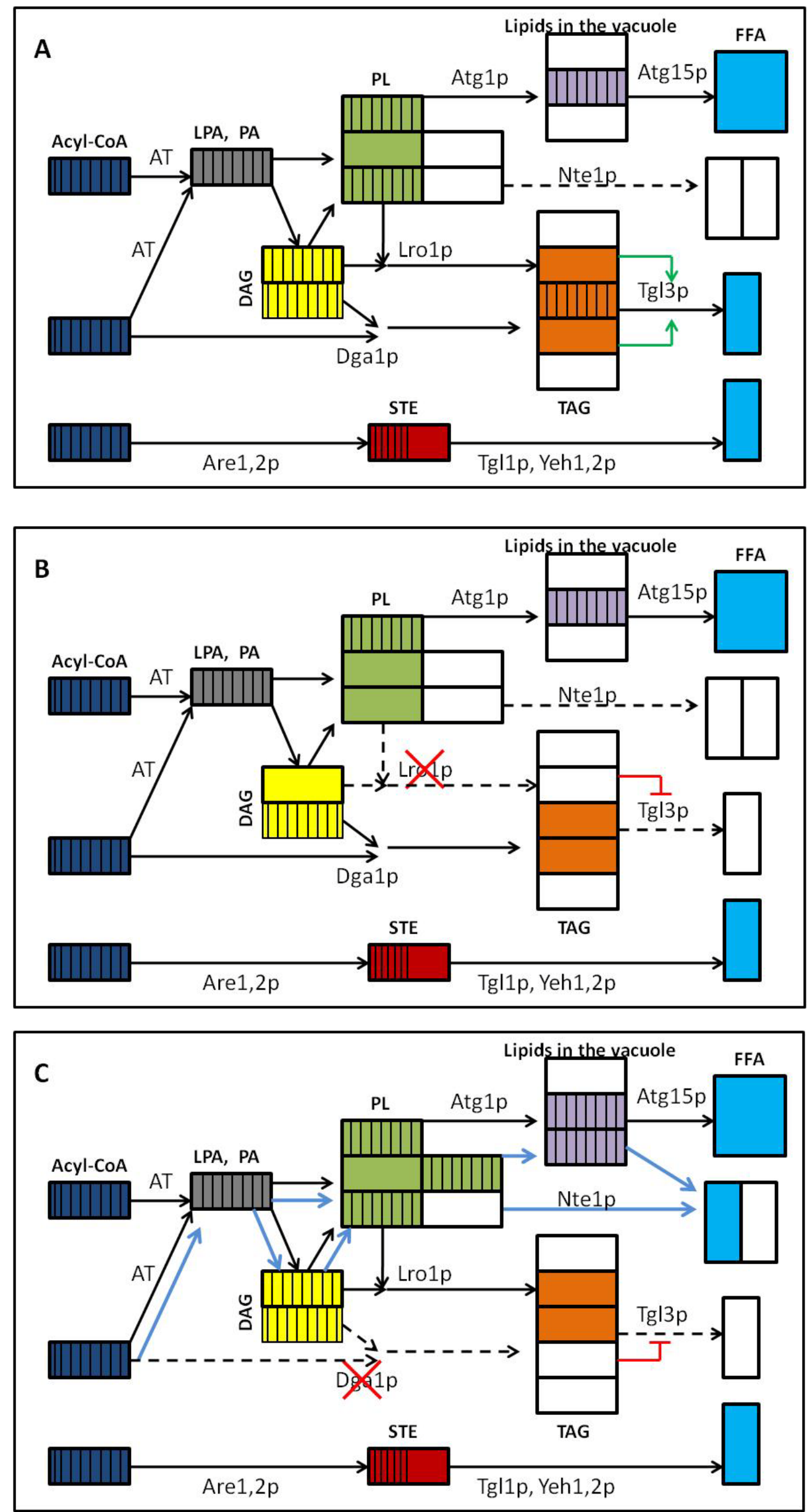

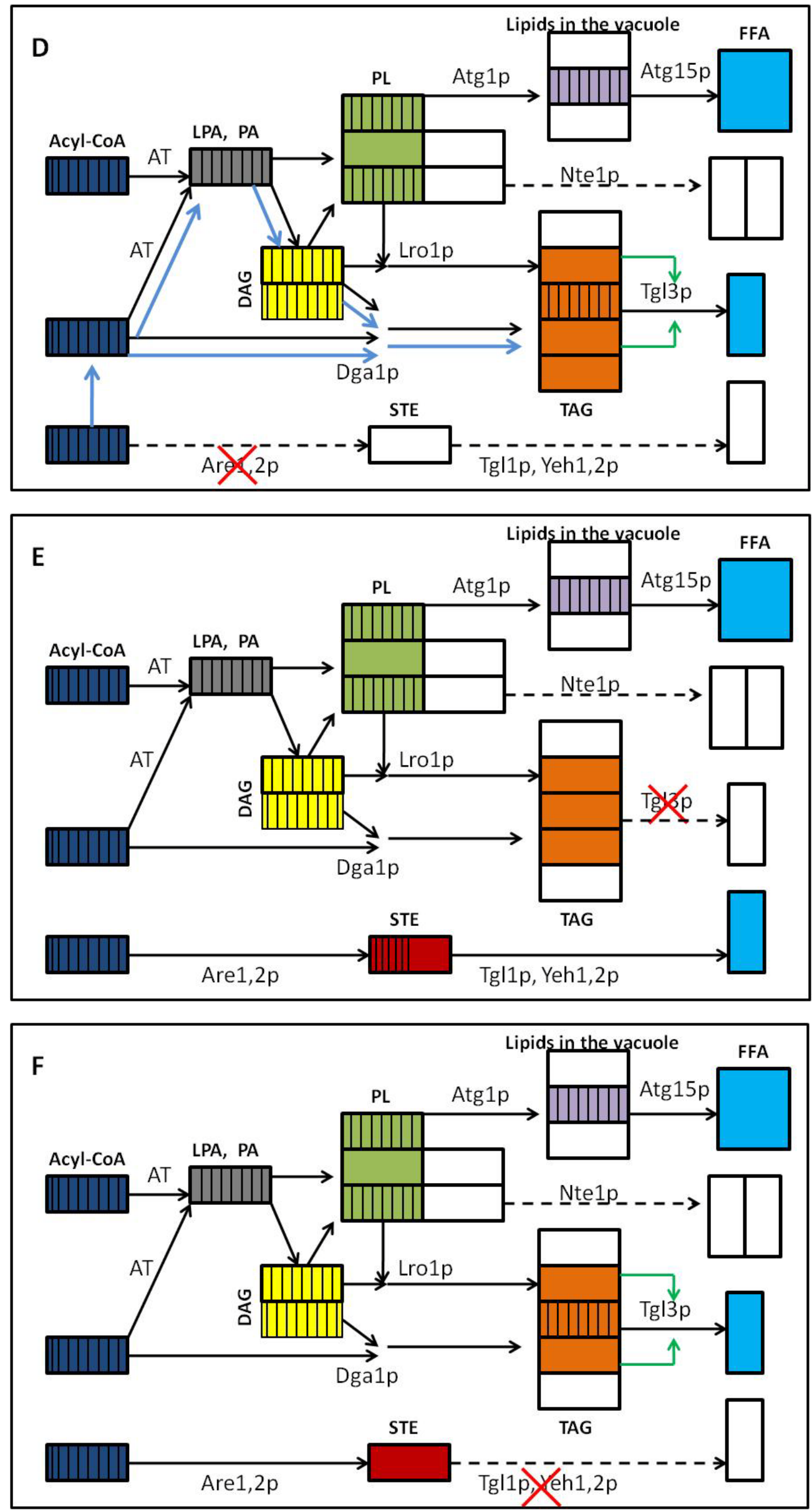

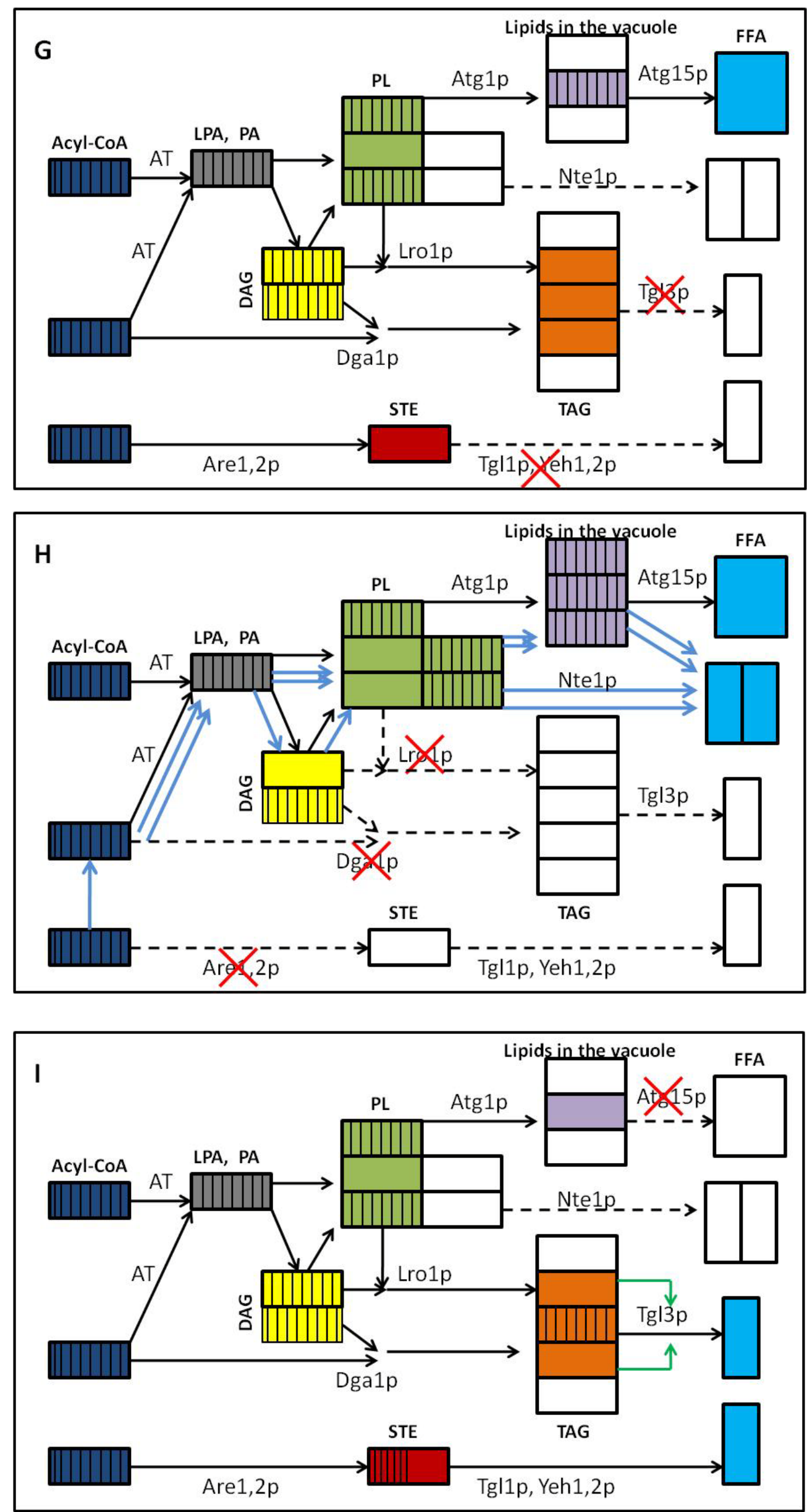

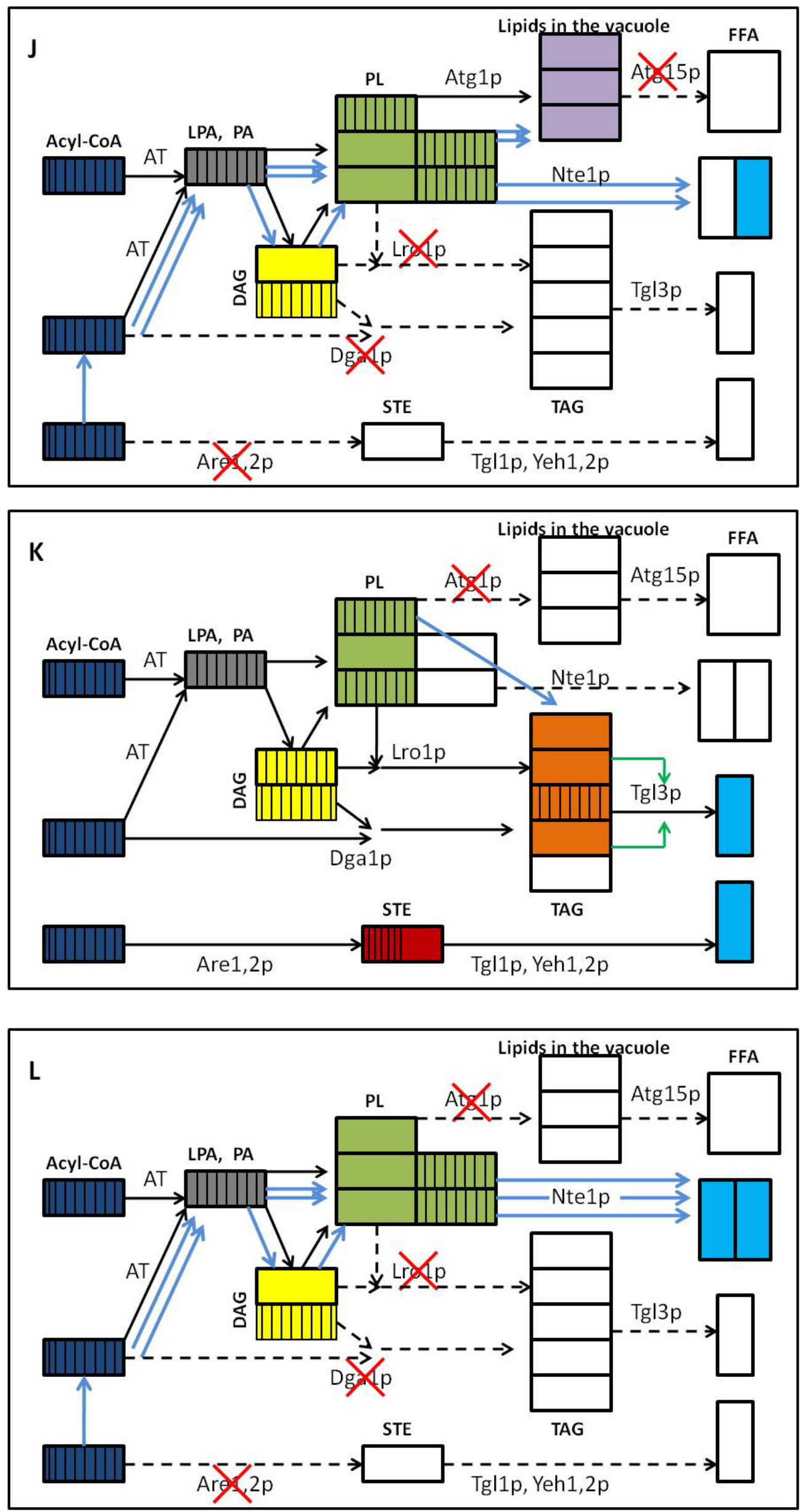

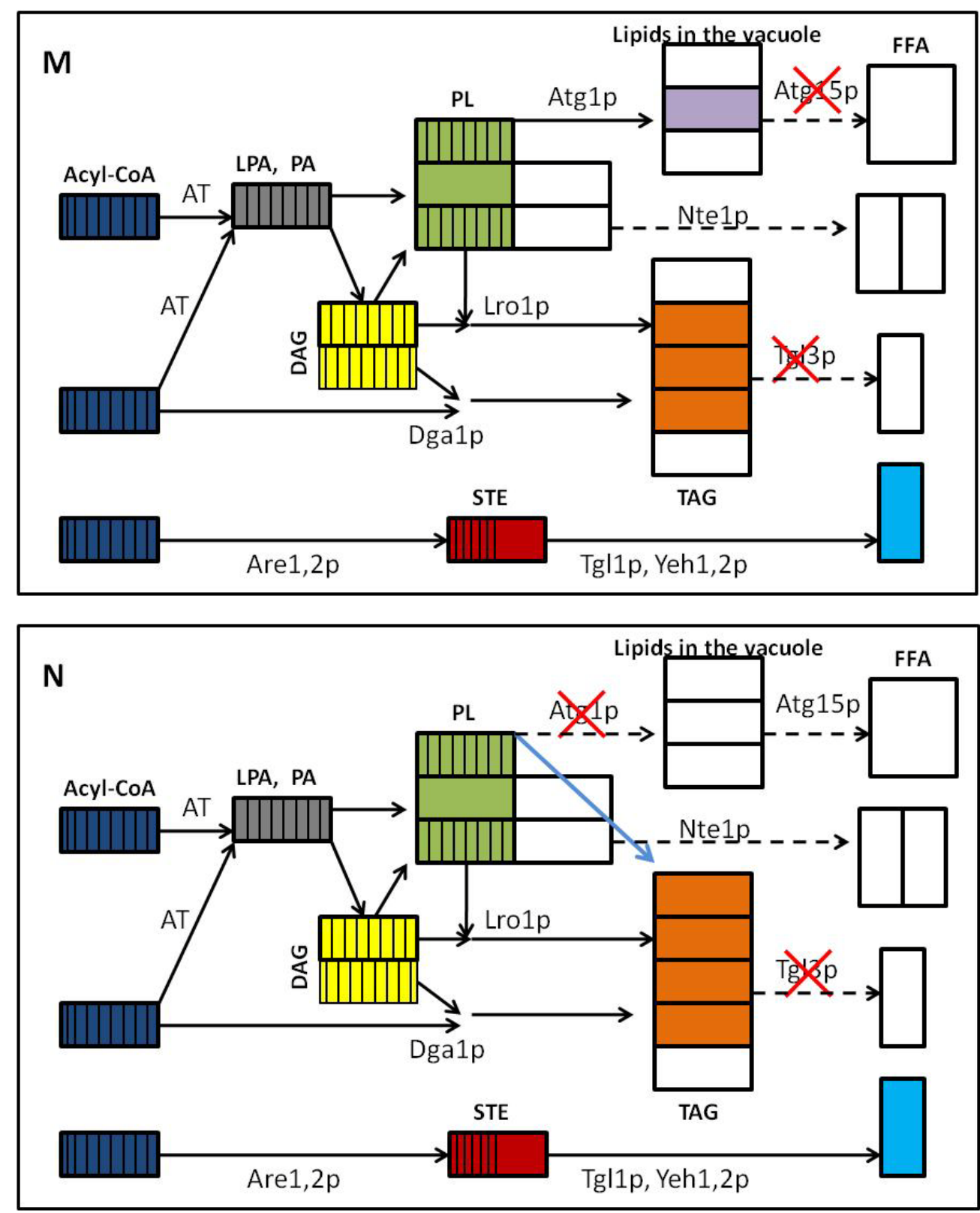

Figure 33. Fatty acid trafficking. Dark blue: Acyl-CoA. Grey: PA and intermediaries of its synthesis. Yellow: DAG. Green: PL. Orange: TAG. Purple: Lipids in the vacuole. Pale blue: FFA.

Colored box: steady pool. Colored box with vertical lines: Pool in transit. This refers to a pool that does not accumulate but is rather converted into a different pool. Empty box: Empty pool, it is kept in the diagram for reference.

Note that the boxes do not pretend to be on scale or represent relative sizes of different pools. The only exception to this is the pool of FFA directly derived from autophagy, which is larger than those derived from TAG or STE mobilization.

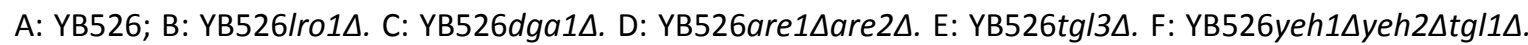

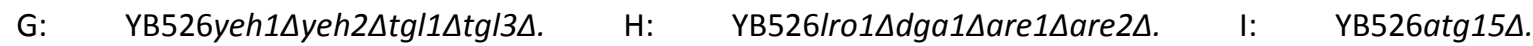

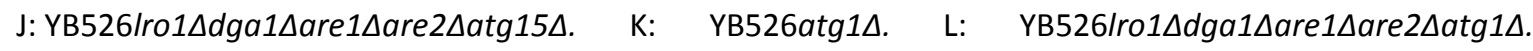

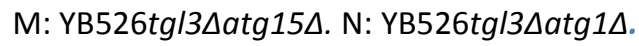




\subsubsection{Atg15p and the exogenous lysolipid metabolism (ELM)}

Two years ago, when Ale1p was identified as the second LPAAT of yeast responsible for the viability of slc1D strains and as a prominent LPLAT central to the Lands cycle for PL remodeling, it was additionally recognized as a key player in the ELM pathway. Even though yeast cells are very tolerant to dramatic variations in their relative content of PL classes, a limit to this tolerance does exist. In particular, a minimum amount of PE must always be present to sustain cell viability. Therefore, the survival of cells rendered unable to convert PS into PE by disruption of the genes PSD1 and PSD2, depends on the availability of an exogenous ethanolamine source. Most commonly this source is presented as ethanolamine itself, which is phosphorylated by the ethanolamine kinase Eki1p and then converted to CDP-ethanolamine for use through the Kennedy pathway by the phosphoethanolamine cytidylyltransferase Ect1p. As an alternative to ethanolamine, psd1 1 psd2 $2 \Delta$ cells can also accept lysoPE as an exogenous source of ethanolamine. In this case, as demonstrated by Riekhof and coworkers (Riekhof et al. 2007), lysoPE is imported through the action of the Dnf1p/Dnf2p/Lem3p complex and acylated by Ale1p directly producing the required PE. However, Riekhof and coworkers found that not only psd1 1 psd2 $2 \Delta$ but also psd1 1 psd2 2 ale $1 \Delta$ cells survive when provided with lysoPE as the single source of ethanolamine. The cited authors proposed that in this case the lysoPE is deacylated to glycerophosphoethanolamine (GPE) and further degraded by a glycerophosphodiesterase to G3P and ethanolamine that can be used in the Kennedy pathway. Accordingly, they observed that a psd1 $\operatorname{psd} 2 \Delta$ ale $1 \Delta$ ect1 $1 \Delta$ strain loses its ability to survive on lysoPE. As it would have been natural to expect, Riekhof and coworkers suggested the phospholipases B, in particular Nte1p, as the most likely candidates to mediate lysoPE deacylation.

At that time we were already confident on our result that the PLB enzymes of yeast are not indispensable for PL deacylation and in fact not involved in it, except under conditions of artificially induced PL overproduction. We had also found the first positive indications of the prominent role Atg15p has in PL deacylation and therefore saw the results and the model of Riekhof and coworkers as an excellent opportunity to test our hypothesis. Cells of W303 background deficient for the genes encoding the phosphatidylserine decarboxylases Psd1p and Psd2p, the LPLAT Ale1p and all the PLB 
enzymes (Plb1p, Plb2p, Plb3p, and Nte1p) were tested for their ability to survive with lysoPE as the only source of ethanolamine. Although these cells have reduced fitness compared to cells of the non auxotrophic wild type and W303plb1 1 plb2 $\Delta p / b 3 \Delta$ nte $1 \Delta a l e 1 \Delta$ strains (figure 32 , right), and also compared to cells provided with ethanolamine instead of lysoPE (figure 32, centre), they remain viable. This confirms the idea that the PLB enzymes are not indispensable for PL deacylation (lysoPE in this case) and places their role in the ELM pathway under doubt (although it does not entirely refute such role). In contrast, cells deficient for ATG15 (in addition to the PLBs, LPLAT and PSD genes) were unable to grow on lysoPE while still healthy on ethanolamine (figure 32, centre and right). This confirms the role of Atg15p (or at least of vacuolar degradation as a whole) in PL deacylation. It also suggests that lysoPE could be a target for Atg15p, a protein for which, as discussed before, no substrate has been identified so far.

\subsubsection{Peroxisomal lipid deacylation}

After the very successful outcome obtained with ATG15, or more precisely with vacuolar degradation in general, as a candidate mechanism for PL deacylation, the role of a second putative lipase of unknown function in the production of FFA was tested through its deletion in YB526 cells: LPX1.

Lpx1p is a peroxisomal matrix protein, strongly induced by supplementation with oleic acid and originally proposed to be a lipase by sequence similarity analysis (Kal et al. 1999; Voss et al. 1997; Schrag and Cygler 1997; Smith et al. 2002). Thoms and coworkers demonstrated, in vitro, phospholipase $A$ and lesser TAG lipase activities for the protein (Thoms et al. 2008). However, its substrate and function, in vivo, remains unknown. Thoms and coworkers showed as well that $I p \times 1 \Delta$ cells display an aberrant peroxisomal morphology, characterized by either membrane invaginations or accumulated vesicles in the peroxisomal lumen. In spite of this, Ipx1 $1 \Delta$ cells clearly retain peroxisomal function.

Deletion of LPX1 on YB526 cells derived in a 30\% decrease in the FFA content (figure 23A). To encounter such a strong contribution to lipid deacylation under our conditions (YPR media) is fairly surprising given that the protein was reported to be induced upon 
culture on oleic acid media; similarly the presence of vesicles (or invaginations) inside the peroxisome upon LPX1 deletion corresponds as well to cultures in oleic acid media. Although, in this regard, it is unclear whether a similar morphological phenotype does or does not occur in complete media. Even more surprising is the fact that this reduction in FFA was not accompanied by an increased content of esterified FA (figure 23B), a behavior observed in other strains presenting a strong FFA decline ${ }^{4}$. This could mean that the loss of Lpx1p activity leads to a signal that inhibits lipid synthesis in general, or alternatively, causes a failure to produce a signal that should promote lipid synthesis. The latter should occur not just at the level of acylation of glycerol backbones, but perhaps at an earlier stage: FA synthesis itself. If this is in fact the case, the FFA "missing" in the YB526/px1A relative to the YB526 strain, cannot be so easily ascribed to the lipase or phospholipase activity of $L p x 1 p$, as this gives us at least two conceivable scenarios: It is possible that Lpx1p does itself mediate the deacylation of a large amount of lipids, accounting for $30 \%$ of the FFA released on the background strain, and that its absence inhibits the production of its own substrate; but it is also possible that Lpx1p mediates only the deacylation of a small amount of lipids, although with a high metabolic impact, and that its absence inhibits the production of a larger amount of lipids that would otherwise be a substrate for degradation through a different pathway.

In the first case, that is if there is an actual peroxisomal pathway for lipid deacylation mediated by Lpx1p and an inhibition in the synthesis of the substrate feeding into this pathway, the effect on the FFA pool caused by the deletion of LPX1 should be at least partly additive with the effects of TGL3 or ATG15 deletions, while the content of bound

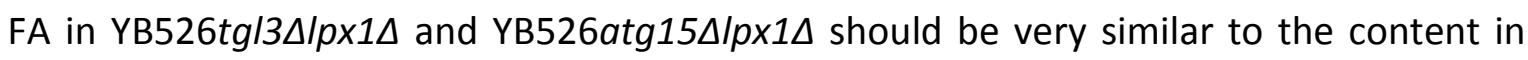

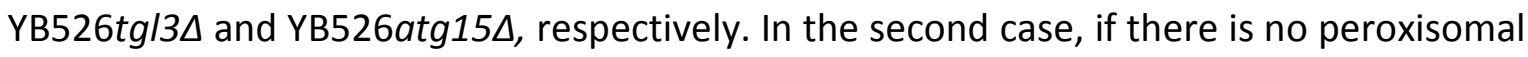
pathway for substantial lipid deacylation and absence of Lpx1p causes a loss of substrate

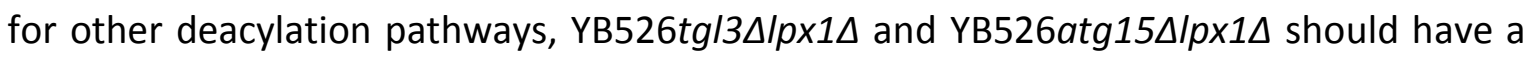

\footnotetext{
${ }^{4}$ In strains capable of NL synthesis this refers to a esterified FA increase respect to the YB526. In strains entirely unable to synthesize NL further mutations leading to a FFA decrease cause an increase of esterified FA with respect to the YB526/ro1 $\Delta d g a 1 \Delta a r e 1 \Delta a r e 2 \Delta$ but not necessarily with respect to the YB526.
} 
very similar FFA content to YB526/px1D and YB526atg15D respectively, ${ }^{5}$ while their

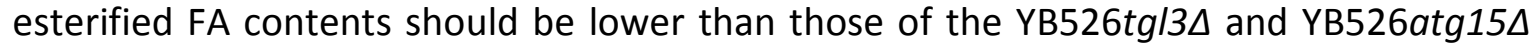
mutants.

Unfortunately, the actual results do not match either case. The size of the FFA pool in the

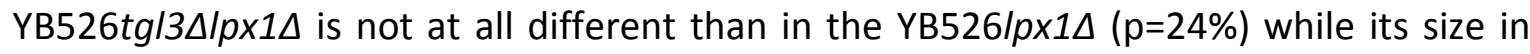

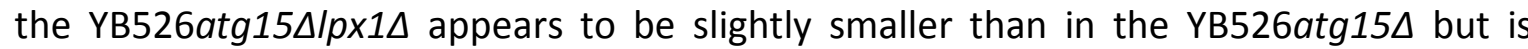
actually not significantly different $(p=81 \%)$ (figure $23 \mathrm{~A}$ ). This behavior of the FFA pool corresponds to the second case described above, but the behavior of the bound FA pool does not: In the enunciated "second case" the simultaneous deletion of LPX1 and either TGL3 or ATG15 should reduce the esterified FA content compared to the single deletions but we actually observe a higher esterified FA content in the YB526tg/3 $\Delta / p \times 1 \Delta$ than in the

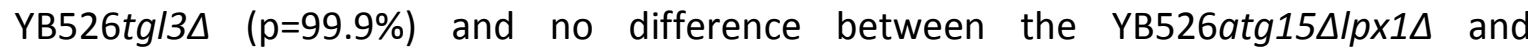
YB526atg15 $\Delta$ mutants ( $p=71 \%$ ) (figure 23B). This actual increase of esterified FA in the

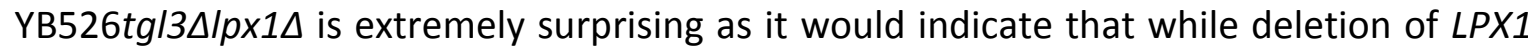
produces an unaccounted reduction in lipid content, its deletion on a $\operatorname{tg} / 3 \Delta$ background actually leads to an unaccounted increase in lipid content. Similarly, the YB526/ro1 $\Delta$ dga1 $\Delta a r e 1 \Delta a r e 2 \Delta / p \times 1 \Delta$ strain presents an increase in esterified FA compared

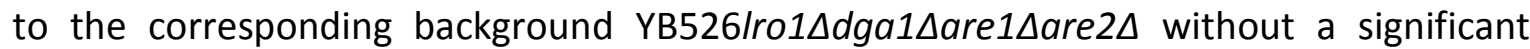
decrease in FFA (figures $23 \mathrm{~A}$ and $\mathrm{B}$ ).

It seems clear that interfering with Lpx1p function affects lipid homeostasis in general, and in particular the balance of the free and esterified FA pools (i.e. the balance of lipid synthesis and degradation), but it is also clear that this effect is more complex than our initial suggestion and perhaps too complex to be resolved with only our current set of data.

In both the cases of the autophagy and NL pathways for PL deacylation, the results presented make up what can be described as an entrance/exit perspective. Blocking the exit points $(\operatorname{tg} / 3 \Delta$, yeh1 $\Delta y e h 2 \Delta \operatorname{tg} / 1 \Delta$ and $\operatorname{atg} 15 \Delta)$ revealed that matter is trapped within

\footnotetext{
${ }^{5}$ The $\operatorname{tg} / 3 \Delta / p \times 1 \Delta$ mutant is compared to the $I p \times 1 \Delta$ while the $\operatorname{atg} 15 \Delta / p \times 1 \Delta$ is compared to the $\operatorname{atg} 15 \Delta$ because the $I p x 1 \Delta$ has slightly less FFA than the $\operatorname{tg} / 3 \Delta$ (although not significantly less, $p=89 \%$ ) but more than the $\operatorname{atg} 15 \Delta$.
} 
the pathways (LP and autophagic vesicles). Blocking the entrance points on the other hand (Iro1 $\Delta$, dga $1 \Delta$, are $1 \Delta a r e 2 \Delta$, and $\operatorname{atg} 1 \Delta)$ allowed us to identify an accumulation of matter in the pools feeding into the deacylation pathways (such as PL in the Iro1 $\Delta$ mutant) or a diversion of matter into either alternative degradative pathways (PL synthesis followed by Nte1p and/or autophagy mediated degradation in dga1 $\Delta$ mutants) or accumulative pathways (TAG synthesis in are $1 \Delta \operatorname{are} 2 \Delta$ and $\operatorname{atg} 1 \Delta$ mutants).

Hoping that it could help us clarify the complex situation encountered upon LPX1 deletion, we deleted a gene the absence of which should altogether prevent lipid transit to the peroxisome. Pex3p is a peroxisomal membrane protein (PMP) required for the correct localization of all other PMPs, its deletion leads to complete absence of peroxisomes or peroxisomal membrane structures (Hettema et al. 2000).

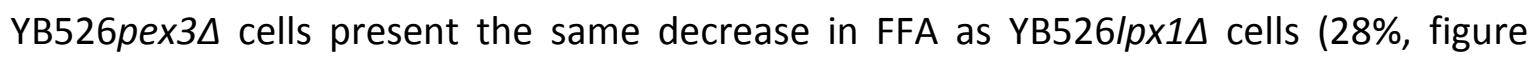
$24 A)$. In this case an increase in esterified FA does occur (27\%, figure 24B). This behavior is what the Iro1 $1 \Delta$ and $\operatorname{atg} 1 \Delta$ strains have taught us to expect from blocking the entrance into a PL deacylation pathway. However, analysis of the YB526pex3 $\Delta$ mutant as well as of further mutants carrying the PEX3 deletion in combination with other mutations is far more complex than the analysis of the strains we have considered so far. The YB526/px1 $\triangle$ mutant has shown us that interference with peroxisomal functions alters lipid homeostasis by what could be a complex signal mediated regulation of the lipid synthesis and degradation rates. In this regard, what should we expect from a pex3 $\Delta$ mutant? Does this hypothetical signal originate from peroxisomal processes and therefore disappears in cells without peroxisomes or does it originate at a different location and is still present in pex3 $\Delta$ cells? In the later case, how is it regulated now? Are pex3 $\Delta$ cells simply equivalent to $I p \times 1 \Delta$ cells in that they have no functional Lpx1p or does the additional failure of other peroxisomal functions give a new metabolic meaning to the absence of Lpx1p? These are questions we cannot currently answer. In consequence there is no point in giving too much thought to the relation of the free and bound FA pools between the YB526 and the YB526pex3 0 or other strains carrying a PEX3 deletion plus additional mutations. What we can of course do, is use the YB526pex3 $\Delta$ strain as a reference to analyze the effect of further mutations on this background. 
If there is a peroxisomal pathway for lipid deacylation the FFA content of

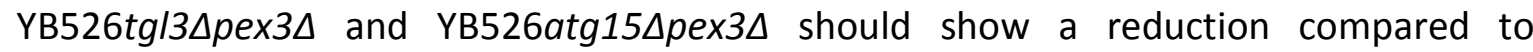

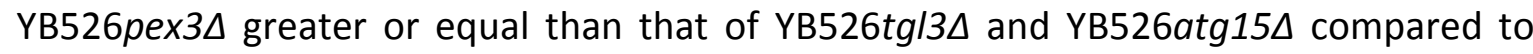
YB526, while the esterified FA content should be higher in the double mutants than in the YB526pex3 $\Delta$. The bound FA pool indeed behaves in this way, although the increase is higher than it would have been expected (figure 24B), but the FFA pool does not: in

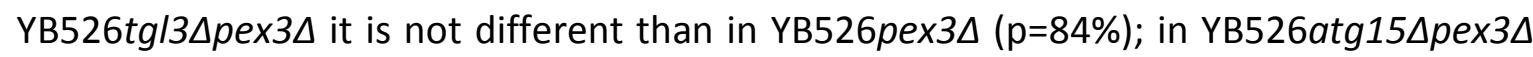
the FFA pool is only reduced by $30 \%$ compared to YB526pex3 $\Delta$ while in YB526atg15 1 the

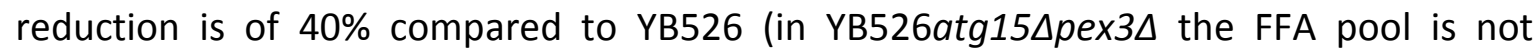
significantly lower than in YB526atg15,$p=87 \%$, figure 24A). The non-additive effect on the FFA pools speaks against the existence of a peroxisomal pathway for deacylation; in this case, however, we cannot claim a reduction in the available substrate for the alternative deacylation pathways, as this would require that YB526tgl3Apex3 $\Delta$ and

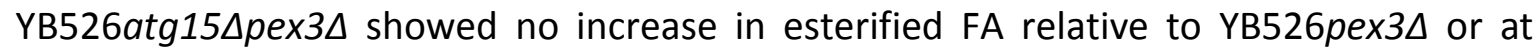

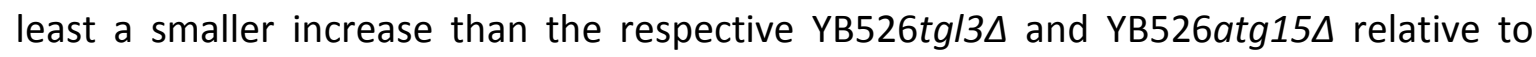
YB526, which is not the case. Most dramatically the content of esterified FA in

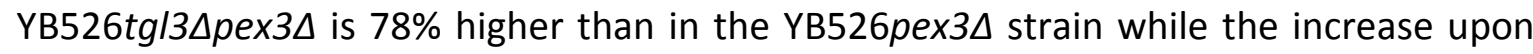
TGL3 deletion on the YB526 background caused only a 41\% raise. Unfortunately, we do

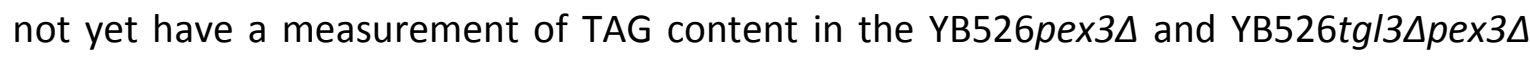
strains that would allow us to establish whether this excess corresponds to TAG or to PL. Since there is no reduction of FFA content accompanying this large increase of esterified FA it would appear that the deletion of both PEX3 and TGL3 but not the deletion of either alone causes augmented lipid synthesis. Recall that the initial "loss" of lipids in the YB526/px1 $\triangle$ was entirely reverted in strains with additional deficiencies in TAG synthesis or mobilization leading to an unaccounted excess of esterified FA, although not as

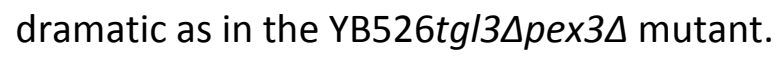

In summary, it is quite clear that a peroxisomal function, involving the activity of Lpx1p, affects the rates of lipid synthesis and degradation. There is very preliminary evidence suggesting that this effect could be a regulatory function. It could perhaps be an integration of the cellular lipid status and in particular of the rate of TAG mobilization, relaying a signal that can repress or promote lipid and FA synthesis. It seems unlikely that 
the lipase activity of Lpx1p could be directly responsible for the deacylation of an amount of lipids large enough to be significant within the sensitivity of our system.

\subsection{Assessment of cell viability}

Given the major metabolic impairments that we impose on our cells through multiple gene deletions and the strong effects that this has on FA recycling and lipid deacylation, and therefore on energy efficiency and membrane homeostasis, the question regarding the fitness of these mutants has often been raised. Our main criterion to assess the well being of a particular culture is its ability to reach an $\mathrm{OD}_{600}$ within the range established as normal through the numerous repetitions of cultures with the reference strain. As shown in table 1 and figure 28 (left), the only strain that fails to pass this criterion is the YB526plc1D. In fact, if the variations observed in $\mathrm{OD}_{600}$ could indicate enhanced or decreased fitness despite being within the normal range by tending to on one or another end of this range, we find that some of the strains with the strongest genetic alterations and the most prominent decreases in FFA production have some of the highest $\mathrm{OD}_{600}$. For example the $\operatorname{tg} / 3 \Delta \operatorname{tg} / 4 \Delta \operatorname{tg} \mid 5 \Delta a \operatorname{tg} 15 \Delta$ strain with one of the lowest FFA contents has an $\mathrm{OD}_{600}$ at the very top of the scale: 7.4. This should not be misinterpreted as an indication that an inverse correlation between FFA content and $\mathrm{OD}_{600}$ must necessarily

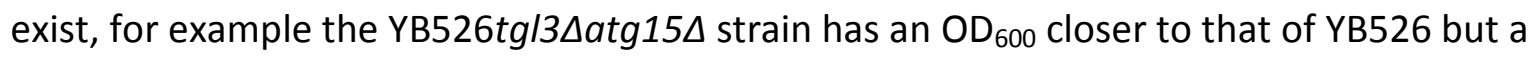

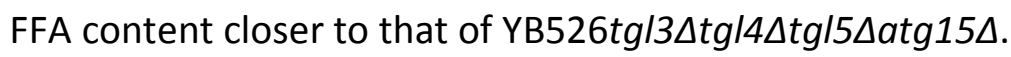

While we consider a normal optical density as sufficient criterion to establish whether the FA content and profiles of different cultures can be directly compared or not, it was decided to address the effect that the introduced mutations could have on cell fitness with a different parameter. Therefore, a colony-forming unit (CFU) assay was carried out for a subset of the strains.

Figure 31 reports the degree of cell viability (DCV) for these strains defined as the fraction of cells, out of the total number present in an aliquot of the culture at late stationary phase, that manage to form colonies upon plating of said aliquot on solid YPD media. Surprisingly the value obtained for the wild type (YB332) is not at all higher than the value obtained for the YB526 strain, indicating that the deletion of the acyl-CoA 
synthetases, and therefore neither the inability to recycle FA nor the presence of large amounts of intracellular FFA, has a deleterious effect on the cells. It must, however, be remembered that while the number of cells per $\mathrm{OD}_{600}$ unit per $\mathrm{ml}$ showed no large variations among strains of YB526 background, the YB332 did present a much higher concentration of cells per $\mathrm{OD}_{600}$ unit (figure 10). The fraction of viable cells (which is the generally accepted experimental parameter) is not affected but the number of viable cells (which might be an important biological parameter) is reduced in the ACS deficient mutant. On an atg15 background, however, the absence of the ACS enzymes does have an effect on the degree of cell viability defined as a fraction: the YB332atg15 $\triangle$ has a considerably higher DCV than the YB526atg15 strain. Given that the DCV for the

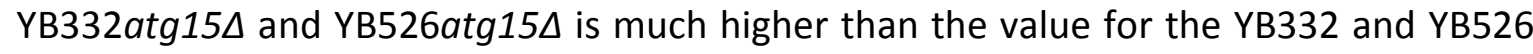
respectively, it would appear that rather than a deleterious effect from the absence of the acyl-CoA synthetases per se, what we have here is a positive effect on DCV by the absence of ATG15 which is maximized in cells with ACS enzymes.

This beneficial effect derived from the absence of ATG15 could, at first, appear to be even more surprising than the lack of difference between the wild type and the YB526 strain. Autophagy is generally considered to extend lifespan under specific culture conditions and it was specifically demonstrated that while some autophagic functions are not required for this effect, intravacuolar vesicle lysis mediated by Atg15p is essential (Tang et al. 2008). However, the apparent contradiction between our results and those presented by Tang and coworkers is derived from the fact that not one but two different criteria for the assessment of lifespan are commonly used. A careful consideration of this indicates that there is not necessarily a contradiction. One type of lifespan measurement, properly referred to as replicative lifespan and which is the one employed by Tang and coworkers, considers the number of times that an individual cell can divide before it dies. The second type of lifespan criteria, normally determined as in our case by the CFU method, is the chronological lifespan. This refers to the length of time that non-dividing cells can survive. Although we employed the CFU method we did not formally carry out a chronological lifespan assessment, since we did not evaluate the variation in survival rate after different extensions of time. We established cell survival after only one specific period of time and therefore refer to the result as "degree of cell viability" specifically 
within our experimental conditions including the length of stationary phase (136 $\mathrm{h}$ total culture) that the cells are subjected to.

Cell population in stationary phase is not homogeneous. Approximately $50 \%$ of the cells in stationary phase are virgin cells (have not budded) while the remaining $50 \%$ have budded once or more; the fraction of the population that a category of cells represents decreases geometrically with the number of times the cell has budded (Ashrafi et al. 1999). Until recently, the working hypothesis assumed that cells from a culture in stationary phase could be generally referred to as quiescent cells, although it was recognized that a (presumably minor) fraction of cells in this stage could be nonquiescent (Gray et al. 2004). Allen and coworkers demonstrated that the fraction of nonquiescent cells in stationary phase does not only exist but in fact corresponds to almost half of the cells at the early stages of the phase. They also found that while the majority of virgin cells fall into the quiescent category, a fraction of them does not. Similarly, most cells with a reproductive history are not quiescent although a considerable number of cells having budded only once are (Allen et al. 2006). Virgin cells have a markedly higher chronological lifespan than cells which have already budded once or more, i.e. a larger fraction of them survives starvation and does so for a longer period (Minois et al. 2009), but, furthermore, the chronological lifespan of non virgin cells decreases as the number of times they budded before entering stationary phase increases (Allen et al. 2006). In a culture of cells with a shorter replicative lifespan the age distribution of the population, not in terms of their proximity to the end of replicative lifespan but in terms of the average number of times cells have budded, will be skewed towards younger cells with a higher chance of surviving starvation. In other words, a cell line that under certain conditions results in a shorter replicative lifespan (atg15 , Tang et al. 2008) could, under related conditions, lead to a culture with a higher chronological lifespan ( $\operatorname{atg} 15 \Delta$ in our measurements). This behavior is not unprecedented. According to Gray and coworkers (Gray et al. 2004) there are several mutations known to affect replicative and chronological aging in opposite ways.

Figure 31 holds further surprising features. The lower end on the DCV scale is occupied by strains impaired in either NL synthesis or NL mobilization: YB526tg/3A,

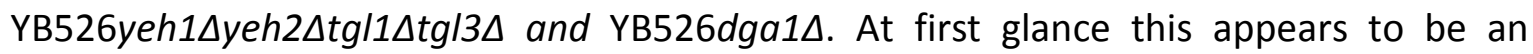


entirely expected result since it is a well known fact that NL mobilization, which must of course be preceded by NL synthesis, provides the cells with a significant advantage in recovering from a period of starvation and resuming rapid replication (Kurat et al. 2006). However, this advantage is supposed to come from the immediate abundance of FA derived from NL mobilization which allows for rapid PL synthesis and membrane formation bypassing de novo FA synthesis; therefore in ACS deficient cells, which are anyway unable to utilize FA derived from lipid mobilization, it should be of no relevance whether these FA can be produced or not. The fact that our ACS deficient strains are, nevertheless, strongly affected by impaired NL synthesis or degradation, suggests that the role of NL mobilization upon exiting stationary phase could not exclusively be the provision of FA for PL synthesis but rather the provision of lipid backbones, in particular DAG which can be either converted to PA or directly used for PL synthesis. In agreement with this idea, the DCV of YB526/ro1 $\triangle$ is extremely high, not only higher than that of the YB526dga1A strain but also higher than that of the YB526 strain. Given that the YB526/ro1 1 strain also has a reduced TAG pool it could be expected to present a behavior similar to the YB526dga1 , however, recall from figure 30 and the discussion in section 5.1.3. that this strain presented a remarkably elevated content of DAG.

The most unexpected result in figure 31, and perhaps the most difficult to explain, is the

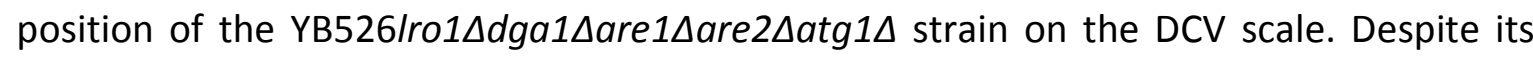
strongly altered genetic content, the major restrictions that these mutations impose on $\mathrm{PL}$ homeostasis, and the fact that the YB526/ro1 1 dga1 and YB526atg1 $\Delta$ strains show a reduced DCV, this mutant has a notoriously higher DCV than the reference strain. Currently, we cannot claim to understand this behavior but we can at least point to one element possibly standing behind it: As discussed at the end of section 5.1.5.2., this mutant contains a higher amount of PL than other strains. It is not unthinkable that a redistribution of such lipids could complement de novo lipid synthesis and facilitate rapid membrane biogenesis to match the requirements of stationary phase exit. 


\subsection{Perspectives and further work}

The analysis of the effect that the introduction of mutations has on the free and general esterified FA pools in ACS deficient cells has already led to the identification of the pathways majorly responsible for lipid deacylation. It has provided insightful information regarding their interaction with each other and regarding the relation of lipid synthesis and degradation with FA homeostasis in a broad sense. This approach, at least as a standalone method, might now be close to exhausting its potential; however, the analysis of a few more mutants could still be pertinent.

The $C L$ specific lipase Cld1p, ironically the only lipase fully identified as involved in PL remodeling, has not yet been tested within our system. Although the fact that the role of Cld1p in PL remodeling has already been established might render its use within our system redundant in some ways, it would nevertheless be interesting to establish the magnitude of its contribution to FFA formation and the interaction (additive/nonadditive) of its activity with that of the other deacylation pathways identified.

The contributions of STE and TAG hydrolysis to the FFA pool were shown to be additive

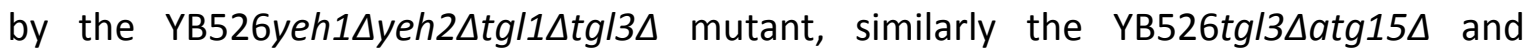

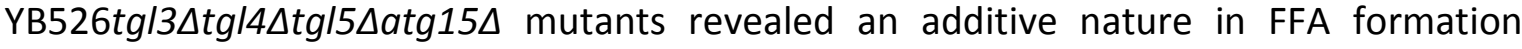
through autophagy and TAG mobilization. It would still be of interest to analyze the FFA content in cells simultaneously deficient for STE hydrolysis and autophagic degradation. Even more relevant, will be the measurement of the FFA pool size in a mutant blocked for all three activities. As indicated before, we expect yeh1 $\Delta y e h 2 \Delta \operatorname{tg} / 1 \Delta \operatorname{tg} / 3 \Delta a \operatorname{tg} 15 \Delta$ and yeh1 $\Delta$ yeh2 $\Delta \operatorname{tg} / 1 \Delta \operatorname{tg} / 3 \Delta \operatorname{tg}|4 \Delta \operatorname{tg}| 5 \Delta a \operatorname{tg} 15 \Delta$ mutants to result in a FFA reduction within 75 and $85 \%$ relative to the background strain. If measurements on these mutants were to match such expectations we could be confident that we have not only identified major pathways for lipid deacylation, but in fact the major pathways. The production of a

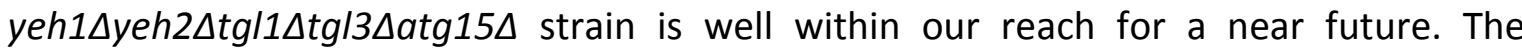
production of a yeh1 $\Delta y e h 2 \Delta \operatorname{tg}|1 \Delta \operatorname{tg}| 3 \Delta \operatorname{tg}|4 \Delta \operatorname{tg}| 5 \Delta a \operatorname{tg} 15 \Delta$ strain, instead, will still require a long period of time.

We have clearly shown that phospholipases B are not involved in constitutive PL deacylation; however, an effect on the FFA pool by deletion of Plb1p, Plb2p and Plb3p 
has not been tested under conditions of enhanced PL synthesis, conditions which showed to be of great relevance in the case of Nte1p. Before introducing further mutations it would be pertinent to verify the effect of enhanced lipid synthesis through media conditions (for example choline supplementation) on the FFA pool of the reference strain and the PLB deficient strains. If differences were to be found, we could proceed by combining DGA1 and ALE1 deletions with single and multiple PLB deletions.

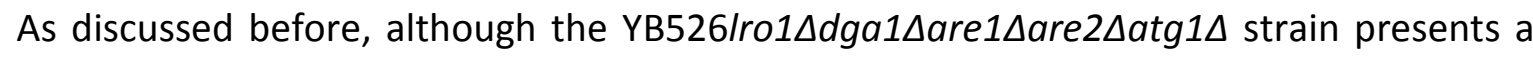
substantially reduced FFA pool compared to the YB526 strain, its FFA content is relatively high compared to strains where the final rather than the initial steps of the deacylation

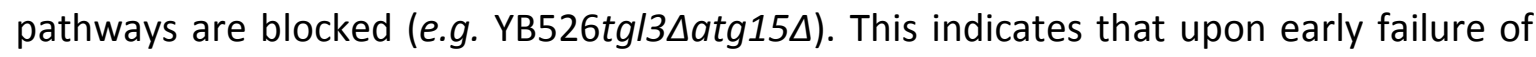
the major deacylation pathways alternative ones must come into play and we have proposed that Nte1p mediated degradation is at least one of them. This requires

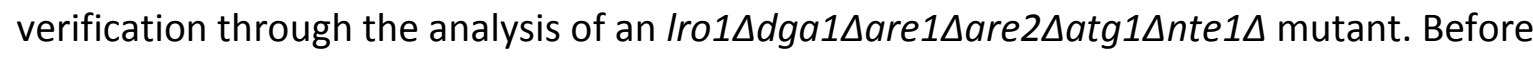
our current model had settled, a number of mutants carrying combined deletions of NTE1, ATG15 and other genes, were produced and analyzed. Those results are not shown since they do not present informative traits or contribute to our discussion; now, however, we know that the deletion combinations should have included ATG1 instead of

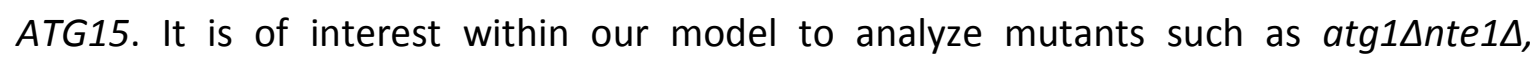

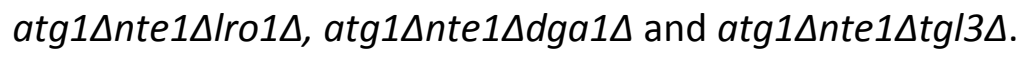

Beyond the remaining mutants indicated above, it is now time to move onto different experimental approaches. As shown in figures 29 and 30, we have already, in few specific cases, proceeded from measuring FFA and general bound FA to measuring FA in specific lipid classes. Although those measurements represent only a minor fraction of our results, their contribution to our understanding of the system and the interpretation of other results is enormous. It therefore seems clear that the natural following step at this stage is the extension of such measurements to a larger subset of our mutant collection.

We proposed, for instance, that deletion of ARE1 and ARE2 leads to an accumulation of TAG. We also proposed that deletion of DGA1 leads to enhanced PL synthesis rather than STE synthesis. Measurements of TAG and STE in the corresponding mutants are therefore in order, and in fact, are currently under way. 
Given the complex role that we propose for Dga1p activity in the modulation of the acylCoA pool and its effect on PL synthesis, an analysis of PL species composition in dga1

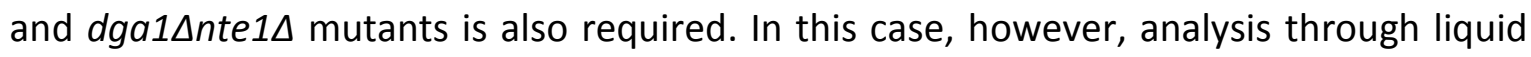
chromatography - mass spectrometry (LC-MS) rather than TLC followed by GC might be necessary, as not only the content of different lipid classes and the content of FA species in particular lipid classes but also the specific distribution of molecular species should be of relevance. This is also true for strains carrying mutations in LRO1, ALE1 and SLC1. In some cases even further specificity in the analysis might be required. For example, while

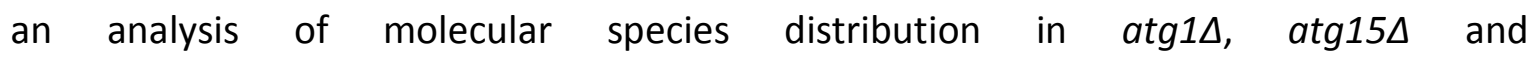
Iro1 1 dga1 1 are $1 \Delta \operatorname{are} 2 \Delta \operatorname{atg} 1 \Delta$ cells would of course be of interest, an analysis of such distributions in specific organelles, chiefly the ER, would be of even greater interest.

Once the experimental approach turns from FFA analysis to the analysis of FA in lipid classes, the opportunity to shift from the ACS deficient background to a wild type background will be open and should of course be taken.

As indicated in the introduction (2.4.), one of the "classical" approaches to PL remodeling, mainly to its reacylation step, is the chase of labeled substrates (FA, backbones and headgroups) and the analysis of their steady state distribution. Now that our analyses of the FFA pool have revealed key players in the deacylation step, the possibility of applying a similar approach for it is open. Analysis of the incorporation of label pulses and/or of label dilution after interrupted label feeding in Iro1 $\Delta$ mutants (on WT background) is the most viable approach to verify its proposed role in PL remodeling. Similar studies in atg15 mutants should clarify whether vacuolar lipid deacylation is a fully degradative or a remodeling pathway.

Some of our analyses refer to different pools of TAG. While the difference could be purely conceptual and have a meaning only in terms of a balance of mass produced through different pathways, the possibility that truly distinct, functionally different pools of TAG could be produced through the activities of Lro1p and Dga1p, was left open. Addressing this problem is enormously difficult, but also extremely relevant since a positive conclusion would be of great impact. An initial approach could consist of feeding ACS deficient cells with FA labeled lysoPE and lysoPC. These species will be acylated and incorporated into the PL pool, eventually some of their FA will reach the Lro1p derived 
TAG pool, but not the Dga1p derived pool. Specifically labeling the Dga1p derived TAG pool, without deleting Lro1p, might be much more challenging, but not necessarily impossible. One possible approach would be analysis of a strain with its G3P and LPA acyl transferases under control of a system as used for heat degradable Plc1p analysis (degron system, sections 3.2.2.7., 3.2.3.5. and 4.3.2.), its acyl-CoA synthetases deleted and one of them (FAA1) reintroduced under GAL1 control. These cells could be grown to a chosen culture stage in raffinose media, their G3P and LPA acyltransferases would then be degraded by increased temperature and galactose supplementation, simultaneously inducing Faa1p expression. Labeled FA fed to the cell at such moment would be activated by Faa1p and utilized by Dga1p, but would not be incorporated into PL and would therefore not become a substrate for Lro1p. After a pulse incorporation of label the cells would be rapidly washed to remove the galactose and any FA not yet imported, incubated for some more minutes at high temperature with addition of glucose to ensure exhaustion of labeled acyl-CoA, and then returned to low temperature. G3P and LPA acyl transferases would then return to normal function but labeled FA released from TAG would not be incorporated into PL as the expression of Faa1p would be repressed by glucose. Having specifically labeled one or the other TAG pool, it would be possible to address the actual existence of differences between them in terms of localization, turnover rate, and distribution of molecular species.

Electron microscopy on YB526 cells revealed an altered ER morphology, which could correspond to FFA accumulation within this organelle (Scharnewski et al. 2008). If such alteration is in fact a consequence of FFA accumulation and not of ACS deficiency itself, the phenotype should be at least partly alleviated in ACS deficient cells with a low FFA

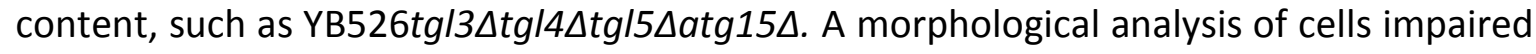
in the initial stages of the deacylation pathways (Iro1 $\Delta a \operatorname{tg} 1 \Delta$ ) would also be of interest, in this case a wild type background could be more convenient than an ACS deficient background.

As mentioned before, the approach used within this work is entirely based on analysis of the steady state FA distribution and lipid composition. Now that we have identified pathways and mechanisms for lipid deacylation and interactions within these pathways, a dynamic analysis is viable. This should include a study of both the time dependant 
evolution of the FFA pool and of (labeled) metabolite progression through different lipid pools in mutants impaired at specific elements of the deacylation network. While FFA analysis must of course be carried out on an ACS deficient background, the tracking of labeled metabolites can be performed on an ACS competent background. 


\section{Conclusions}

The phospholipases $\mathrm{B}, \mathrm{Plb} 1 \mathrm{p}, \mathrm{Plb} 2 \mathrm{p}, \mathrm{Plb} 3 \mathrm{p}$ and Nte1p are not indispensable for PL deacylation and, in fact, are not involved in constitutive PL deacylation under the conditions of our study. In addition to excluding them from constitutive PL degradation, this also means that the known PLB enzymes from $S$. cerevisiae are not involved in PL remodeling, a process for which they have been often considered as plausible candidates.

However, at least Nte1p is involved in PL deacylation under conditions of altered (enhanced) PL synthesis. The activity of Nte1p, and perhaps also that of the other PLBs, could become constitutively relevant when alternative activities for PL degradation, PL remodeling and, in general, the regulation of PL species composition are compromised.

In contrast to the PLB enzymes, the PDAT Lro1p is clearly involved in FA homeostasis and emerges as the most likely mediator of PL remodeling. Lro1p activity affects the FFA pool in ACS deficient cells (and therefore the acyl-CoA pool and PL synthesis in wild type cells), the PL pool (particularly PE), the NL pool (TAG) and a pool of PL and NL precursors (DAG). Far beyond its role as a synthase of TAG for metabolite storage, Lro1p affects the entire equilibrium of FA and glycerolipid metabolism.

Beyond the activity of Lro1p, NL metabolism as a whole is central to FA homeostasis and the regulation of $\mathrm{PL}$ species composition. The role of $\mathrm{NL}$ synthesis in the modulation (detoxification) of an acyl-CoA pool distorted by import of FA from the medium has been previously demonstrated. Our results further indicate that NL synthesis, most prominently through the activity of Dga1p, serves to modulate the composition of the acyl-CoA pool derived from endogenous sources and, therefore, that it can indirectly affect the FA species distribution resulting from PL synthesis. While NL mobilization serves a clear and well established role in providing metabolites for PL synthesis, and therefore in uncoupling PL synthesis from FA synthesis through a positive regulation of the acyl-CoA pool, it now becomes apparent that the opposite effect, uncoupling PL synthesis from FA availability through a negative regulation of the acyl-CoA pool, is also a 
relevant task, and not just a side effect, of NL synthesis. Furthermore, PL remodeling mediated by Lro1p instead of a PLB or purely PLA enzyme, allows for this process to occur without a direct effect on FFA production, therefore uncoupling the size and composition of the acyl-CoA pool from PL remodeling.

We have identified a very interesting behavior in TAG mobilization. While TAG degradation mediated by Tgl3p constitutes a prominent source of FFA in YB526 cells (and therefore a source of acyl-CoA in WT cells), no FFA production through Tgl3p activity takes place in YB526 cells when either Lro1p or Dga1p are absent. This indicates that, under our experimental conditions, the independent biosynthetic activities of Lro1p and Dga1p could be coordinately involved in the regulation of TAG mobilization, providing the cell with the possibility of integrating the effect of the acyl-CoA status on Dga1p activity with the effect of PL species composition status on Lro1p activity, and responding with TAG mobilization that is not exclusively tied, neither quantitatively nor qualitatively, to the magnitude or profile of TAG synthesized by either of the pathways alone.

In this study, autophagy was identified as the quantitatively most prominent single contributor to lipid deacylation in ACS deficient S. cerevisiae cells. While this clearly indicates that autophagy is central to PL and FA homeostasis, our results further suggest that, despite being a bulk process, lipid sequestration for autophagic degradation is not entirely indifferent to the species composition of its substrate. This means that, in addition to a quantitative regulation of PL content, FFA generation and, in WT cells, acylCoA availability, autophagy has the potential to affect the species composition of the cellular lipid content.

Autophagy, TAG metabolism and STE metabolism constitute independent pathways for lipid deacylation, FFA production, regulation of lipid content and regulation of lipid species composition. However, despite being independent, interactions between these pathways take place. Of particular interest is the fact that impaired autophagic sequestration leads to augmented NL synthesis, while impaired NL synthesis leads to enhanced lipid degradation through autophagy.

Peroxisomal function in general, and the activity of the peroxisomal lipase Lpx1p in particular, have a substantial effect on FFA production and on cellular lipid content. In 
this case, however, the function in question cannot be simply understood in terms of lipid deacylation. Although further research is clearly required before confident statements can be made on this matter, we can venture to say that the results obtained so far appear to reflect the relay, through peroxisomal function, of a complex effect from the rate of TAG mobilization on the rates of FA and lipid synthesis. 



\section{Bibliography}

Abeliovich H, Zhang C, Dunn WA, Shokat KM, Klionsky DJ. 2003. Chemical genetic analysis of Apg1 reveals a non-kinase role in the induction of autophagy. Mol. Biol. Cell 14: 477-90.

Abumrad N, Coburn C, Ibrahimi A. 1999. Membrane proteins implicated in long-chain fatty acid uptake by mammalian cells: CD36, FATP and FABPm. Biochim. Biophys. Acta 1441: 4-13.

Al-Feel W, Chirala SS, Wakil SJ. 1992. Cloning of the yeast FAS3 gene and primary structure of yeast acetylCoA carboxylase. Proc. Natl. Acad. Sci. U.S.A. 89: 4534-8.

Allen C, Büttner S, Aragon AD, Thomas JA, Meirelles O, Jaetao JE, Benn D, Ruby SW, Veenhuis M, Madeo F, Werner-Washburne M. 2006. Isolation of quiescent and nonquiescent cells from yeast stationary-phase cultures. J. Cell Biol. 174: 89-100.

Ambroziak J, Henry SA. 1994. INO2 and INO4 gene products, positive regulators of phospholipid biosynthesis in Saccharomyces cerevisiae, form a complex that binds to the INO1 promoter. J. Biol. Chem. 269: 15344-9.

Ashrafi K, Sinclair D, Gordon JI, Guarente L. 1999. Passage through stationary phase advances replicative aging in Saccharomyces cerevisiae. Proc. Natl. Acad. Sci. U.S.A. 96: 9100-5.

Athenstaedt K, Daum G. 1997. Biosynthesis of phosphatidic acid in lipid particles and endoplasmic reticulum of Saccharomyces cerevisiae. J. Bacteriol. 179: 7611-7616.

Athenstaedt K, Daum G. 1999. Phosphatidic acid, a key intermediate in lipid metabolism. Eur. J. Biochem. 266: 1-16.

Athenstaedt K, Daum G. 2000. 1-Acyldihydroxyacetone-phosphate reductase (Ayr1p) of the yeast Saccharomyces cerevisiae encoded by the open reading frame YIL124W is a major component of lipid particles. J. Biol. Chem. 275: 235-40.

Athenstaedt K, Daum G. 2003. YMR313c/TGL3 encodes a novel triacylglycerol lipase located in lipid particles of Saccharomyces cerevisiae. J. Biol. Chem. 278: 23317-23.

Athenstaedt K, Daum G. 2005. Tg|4p and Tgl5p, two triacylglycerol lipases of the yeast Saccharomyces cerevisiae are localized to lipid particles. J. Biol. Chem. 280: 37301-9.

Athenstaedt K, Weys S, Paltauf F, Daum G. 1999a. Redundant systems of phosphatidic acid biosynthesis via acylation of glycerol-3-phosphate or dihydroxyacetone phosphate in the yeast Saccharomyces cerevisiae. J. Bacteriol. 181: 1458-63.

Athenstaedt K, Zweytick D, Jandrositz A, Kohlwein SD, Daum G. 1999b. Identification and characterization of major lipid particle proteins of the yeast Saccharomyces cerevisiae. J. Bacteriol. 181: 6441-8.

Atkinson K, Fogel S, Henry SA. 1980a. Yeast mutant defective in phosphatidylserine synthesis. J. Biol. Chem 255: 6653-61.

Atkinson KD, Jensen B, Kolat AI, Storm EM, Henry SA, Fogel S. 1980b. Yeast mutants auxotrophic for choline or ethanolamine. J. Bacteriol. 141: 558-64. 
Baba M, Osumi M, Scott SV, Klionsky DJ, Ohsumi Y. 1997. Two distinct pathways for targeting proteins from the cytoplasm to the vacuole/lysosome. J. Cell Biol. 139: 1687-95.

Bachhawat N, Ouyang Q, Henry SA. 1995. Functional characterization of an inositol-sensitive upstream activation sequence in yeast. $\mathrm{A}$ cis-regulatory element responsible for inositol-choline mediated regulation of phospholipid biosynthesis. J. Biol. Chem. 270: 25087-95.

Bae-Lee MS, Carman GM. 1984. Phosphatidylserine synthesis in Saccharomyces cerevisiae. Purification and characterization of membrane-associated phosphatidylserine synthase. J. Biol. Chem. 259: 10857-62.

Baehrecke EH. 2005. Autophagy: dual roles in life and death? Nat. Rev. Mol. Cell Biol. 6: 505-10.

Bar-Joseph Z, Gerber GK, Lee TI, Rinaldi NJ, Yoo JY, Robert F, Gordon DB, Fraenkel E, Jaakkola TS, Young RA, Gifford DK. 2003. Computational discovery of gene modules and regulatory networks. Nat. Biotechnol. 21: $1337-42$.

Beaudoin F, Gable K, Sayanova O, Dunn T, Napier JA. 2002. A Saccharomyces cerevisiae gene required for heterologous fatty acid elongase activity encodes a microsomal beta-keto-reductase. J. Biol. Chem. 277: 11481-8.

Becker GW, Lester RL. 1977. Changes in phospholipids of Saccharomyces cerevisiae associated with inositol-less death. J. Biol. Chem. 252: 8684-91.

Becker GW, Lester RL. 1980. Biosynthesis of phosphoinositol-containing sphingolipids from phosphatidylinositol by a membrane preparation from Saccharomyces cerevisiae. J. Bacteriol. 142: 747-54.

Benghezal M, Roubaty C, Veepuri V, Knudsen J, Conzelmann A. 2007. SLC1 and SLC4 encode partially redundant acyl-coenzyme A 1-acylglycerol-3-phosphate O-acyltransferases of budding yeast. J. Biol. Chem. 282: 30845-55.

Beranek A, Rechberger G, Knauer H, Wolinski H, Kohlwein SD, Leber R. 2009. Identification of a cardiolipin-specific phospholipase encoded by the gene CLD1 (YGR110W) in yeast. J. Biol. Chem. 284: 11572-8.

Bergamini E, Cavallini G, Donati A, Gori Z. 2003. The anti-ageing effects of caloric restriction may involve stimulation of macroautophagy and lysosomal degradation, and can be intensified pharmacologically. Biomed. Pharmacother. 57: 203-8.

Berk PD, Stump DD. 1999. Mechanisms of cellular uptake of long chain free fatty acids. Mol. Cell. Biochem. 192: 17-31.

Bernales S, McDonald KL, Walter P. 2006. Autophagy counterbalances endoplasmic reticulum expansion during the unfolded protein response. PLoS Biol. 4: e423.

Bernales S, Schuck S, Walter P. 2007. ER-phagy: selective autophagy of the endoplasmic reticulum. Autophagy 3: 285-7.

Bessoule JJ, Lessire R, Rigoulet M, Guerin B, Cassagne C. 1988. Localization of the synthesis of very-longchain fatty acid in mitochondria from Saccharomyces cerevisiae. Eur. J. Biochem. 177: 207-11.

Birner R, Bürgermeister M, Schneiter R, Daum G. 2001. Roles of phosphatidylethanolamine and of its several biosynthetic pathways in Saccharomyces cerevisiae. Mol. Biol. Cell 12: 997-1007.

Black PN, DiRusso CC. 2003. Transmembrane movement of exogenous long-chain fatty acids: proteins, enzymes, and vectorial esterification. Microbiol. Mol. Biol. Rev. 67: 454-72. 
Black PN, DiRusso CC. 2007. Yeast acyl-CoA synthetases at the crossroads of fatty acid metabolism and regulation. Biochim. Biophys. Acta 1771: 286-98.

Blobel F, Erdmann R. 1996. Identification of a yeast peroxisomal member of the family of AMP-binding proteins. Eur. J. Biochem. 240: 468-76.

Blommaart EF, Krause U, Schellens JP, Vreeling-Sindelárová H, Meijer AJ. 1997. The phosphatidylinositol 3-kinase inhibitors wortmannin and LY294002 inhibit autophagy in isolated rat hepatocytes. Eur. J. Biochem. 243: 240-6.

Boeke JD, LaCroute F, Fink GR. 1984. A positive selection for mutants lacking orotidine-5'-phosphate decarboxylase activity in yeast: 5-fluoro-orotic acid resistance. Mol. Gen. Genet. 197: 345-346.

Bossie MA, Martin CE. 1989. Nutritional regulation of yeast delta-9 fatty acid desaturase activity. J. Bacteriol. 171: 6409-13.

Bosson R, Jaquenoud M, Conzelmann A. 2006. GUP1 of Saccharomyces cerevisiae encodes an Oacyltransferase involved in remodeling of the GPI anchor. Mol. Biol. Cell 17: 2636-45.

Boumann HA, Damen MJ, Versluis C, Heck AJ, de Kruijff B, de Kroon Al. 2003. The two biosynthetic routes leading to phosphatidylcholine in yeast produce different sets of molecular species. Evidence for lipid remodeling. Biochemistry 42: 3054-9.

Boumann HA, Gubbens J, Koorengevel MC, Oh C, Martin CE, Heck AJ, Patton-Vogt J, Henry SA, de Kruijff B, de Kroon Al. 2006. Depletion of phosphatidylcholine in yeast induces shortening and increased saturation of the lipid acyl chains: evidence for regulation of intrinsic membrane curvature in a eukaryote. Mol. Biol. Cell 17: 1006-17.

Bradshaw PC, Jung DW, Pfeiffer DR. 2001. Free fatty acids activate a vigorous $\mathrm{Ca}^{2+}: 2 \mathrm{H}^{+}$antiport activity in yeast mitochondria. J. Biol. Chem 276: 40502-9.

Brody S, Oh C, Hoja U, Schweizer E. 1997. Mitochondrial acyl carrier protein is involved in lipoic acid synthesis in Saccharomyces cerevisiae. FEBS Lett. 408: 217-20.

Bryant NJ, Piper RC, Weisman LS, Stevens TH. 1998. Retrograde traffic out of the yeast vacuole to the TGN occurs via the prevacuolar/endosomal compartment. J. Cell Biol. 142: 651-63.

Bürgermeister M, Birner-Grünberger R, Heyn M, Daum G. 2004a. Contribution of different biosynthetic pathways to species selectivity of aminoglycerophospholipids assembled into mitochondrial membranes of the yeast Saccharomyces cerevisiae. Biochim. Biophys. Acta 1686: 148-60.

Bürgermeister M, Birner-Grünberger R, Nebauer R, Daum G. 2004b. Contribution of different pathways to the supply of phosphatidylethanolamine and phosphatidylcholine to mitochondrial membranes of the yeast Saccharomyces cerevisiae. Biochim. Biophys. Acta 1686: 161-8.

Carman GM, Han G. 2007. Regulation of phospholipid synthesis in Saccharomyces cerevisiae by zinc depletion. Biochim. Biophys. Acta 1771: 322-30.

Carman GM, Han G. 2009a. Regulation of phospholipid synthesis in yeast. J. Lipid Res. 50 Suppl: S69-73.

Carman GM, Han G. 2009b. Phosphatidic acid phosphatase, a key enzyme in the regulation of lipid synthesis. J. Biol. Chem. 284: 2593-7.

Carman GM, Henry SA. 1989. Phospholipid biosynthesis in yeast. Annu. Rev. Biochem. 58: 635-69. 
Carman GM, Henry SA. 1999. Phospholipid biosynthesis in the yeast Saccharomyces cerevisiae and interrelationship with other metabolic processes. Prog. Lipid Res. 38: 361-99.

Carman GM, Henry SA. 2007. Phosphatidic acid plays a central role in the transcriptional regulation of glycerophospholipid synthesis in Saccharomyces cerevisiae. J. Biol. Chem. 282: 37293-7.

Carman GM, Zeimetz GM. 1996. Regulation of phospholipid biosynthesis in the yeast Saccharomyces cerevisiae. J. Biol. Chem. 271: 13293-6.

Chang Y, Carman GM. 2006. Casein kinase II phosphorylation of the yeast phospholipid synthesis transcription factor Opi1p. J. Biol. Chem. 281: 4754-61.

Chang Y, Carman GM. 2008. CTP synthetase and its role in phospholipid synthesis in the yeast Saccharomyces cerevisiae. Prog. Lipid Res. 47: 333-9.

Chang SC, Heacock PN, Clancey CJ, Dowhan W. 1998a. The PEL1 gene (renamed PGS1) encodes the phosphatidylglycero-phosphate synthase of Saccharomyces cerevisiae. J. Biol. Chem. 273: 9829-36.

Chang SC, Heacock PN, Mileykovskaya E, Voelker DR, Dowhan W. 1998b. Isolation and characterization of the gene (CLS1) encoding cardiolipin synthase in Saccharomyces cerevisiae. J. Biol. Chem. 273: 14933-41.

Chen M, Hancock LC, Lopes JM. 2007b. Transcriptional regulation of yeast phospholipid biosynthetic genes. Biochim. Biophys. Acta 1771: 310-21.

Chen Q, Kazachkov M, Zheng Z, Zou J. 2007a. The yeast acylglycerol acyltransferase LCA1 is a key component of Lands cycle for phosphatidylcholine turnover. FEBS Lett. 581: 5511-6.

Cheong H, Nair U, Geng J, Klionsky DJ. 2008. The Atg1 kinase complex is involved in the regulation of protein recruitment to initiate sequestering vesicle formation for nonspecific autophagy in Saccharomyces cerevisiae. Mol. Biol. Cell 19: 668-81.

Chi A, Huttenhower C, Geer LY, Coon JJ, Syka JE, Bai DL, Shabanowitz J, Burke DJ, Troyanskaya OG, Hunt DF. 2007. Analysis of phosphorylation sites on proteins from Saccharomyces cerevisiae by electron transfer dissociation (ETD) mass spectrometry. Proc. Natl. Acad. Sci. U.S.A. 104: 2193-8.

Chirala SS. 1992. Coordinated regulation and inositol-mediated and fatty acid-mediated repression of fatty acid synthase genes in Saccharomyces cerevisiae. Proc. Natl. Acad. Sci. U.S.A. 89: 10232-6.

Chirala SS, Kuziora MA, Spector DM, Wakil SJ. 1987. Complementation of mutations and nucleotide sequence of FAS1 gene encoding beta subunit of yeast fatty acid synthase. J. Biol. Chem. 262: 4231-40.

Chirala SS, Zhong Q, Huang W, Al-Feel W. 1994. Analysis of FAS3/ACC regulatory region of Saccharomyces cerevisiae: identification of a functional $\mathrm{UAS}_{\mathrm{INO}}$ and sequences responsible for fatty acid mediated repression. Nucleic Acids Res. 22: 412-8.

Cho RJ, Campbell MJ, Winzeler EA, Steinmetz L, Conway A, Wodicka L, Wolfsberg TG, Gabrielian AE, Landsman D, Lockhart DJ, Davis RW. 1998. A genome-wide transcriptional analysis of the mitotic cell cycle. Mol. Cell 2: 65-73.

Choi JY, Martin CE. 1999. The Saccharomyces cerevisiae FAT1 gene encodes an acyl-CoA synthetase that is required for maintenance of very long chain fatty acid levels. J. Biol. Chem 274: 4671-83.

Clancey CJ, Chang SC, Dowhan W. 1993. Cloning of a gene (PSD1) encoding phosphatidylserine decarboxylase from Saccharomyces cerevisiae by complementation of an Escherichia coli mutant. J. Biol. Chem. 268: 24580-90. 
Conzelmann A, Fankhauser C, Desponds C. 1990. Myoinositol gets incorporated into numerous membrane glycoproteins of Saccharomyces cerevisiae; incorporation is dependent on phosphomannomutase (sec53). EMBO J. 9: 653-61.

Cottrell M, Viljoen BC, Kock JL, Lategan PM. 1986. The long-chain fatty acid compositions of species representing the genera Saccharomyces, Schwanniomyces and Lipomyces. J. Gen. Microbiol. 132: 24012403.

Czabany T, Athenstaedt K, Daum G. 2007. Synthesis, storage and degradation of neutral lipids in yeast. Biochim. Biophys. Acta 1771: 299-309.

Czabany T, Wagner A, Zweytick D, Lohner K, Leitner E, Ingolic E, Daum G. 2008. Structural and biochemical properties of lipid particles from the yeast Saccharomyces cerevisiae. J. Biol. Chem. 283: 17065-74.

Dahlqvist A, Stahl U, Lenman M, Banas A, Lee M, Sandager L, Ronne H, Stymne S. 2000. Phospholipid:diacylglycerol acyltransferase: an enzyme that catalyzes the acyl-CoA-independent formation of triacylglycerol in yeast and plants. Proc. Natl. Acad. Sci. U.S.A. 97: 6487-92.

Daum G, Lees ND, Bard M, Dickson R. 1998. Biochemistry, cell biology and molecular biology of lipids of Saccharomyces cerevisiae. Yeast 14: 1471-1510.

Daum G, Wagner A, Czabany T, Athenstaedt K. 2007. Dynamics of neutral lipid storage and mobilization in yeast. Biochimie 89: 243-8.

de Kroon Al. 2007. Metabolism of phosphatidylcholine and its implications for lipid acyl chain composition in Saccharomyces cerevisiae. Biochim. Biophys. Acta 1771: 343-52.

Dephoure N, Howson RW, Blethrow JD, Shokat KM, O'Shea EK. 2005. Combining chemical genetics and proteomics to identify protein kinase substrates. Proc. Natl. Acad. Sci. U.S.A. 102: 17940-5.

DiRusso CC, Black PN. 1999. Long-chain fatty acid transport in bacteria and yeast. Paradigms for defining the mechanism underlying this protein-mediated process. Mol. Cell. Biochem. 192: 41-52.

Dietz M, Heyken W, Hoppen J, Geburtig S, Schüller H. 2003. TFIIB and subunits of the SAGA complex are involved in transcriptional activation of phospholipid biosynthetic genes by the regulatory protein Ino2 in the yeast Saccharomyces cerevisiae. Mol. Microbiol. 48: 1119-30.

Dittrich F, Zajonc D, Hühne K, Hoja U, Ekici A, Greiner E, Klein H, Hofmann J, Bessoule J, Sperling P, Schweizer E. 1998. Fatty acid elongation in yeast--biochemical characteristics of the enzyme system and isolation of elongation-defective mutants. Eur. J. Biochem. 252: 477-85.

Dorn BR, Dunn WA, Progulske-Fox A. 2002. Bacterial interactions with the autophagic pathway. Cell. Microbiol. 4: 1-10.

Dowd SR, Bier ME, Patton-Vogt JL. 2001. Turnover of phosphatidylcholine in Saccharomyces cerevisiae. The role of the CDP-choline pathway. J. Biol. Chem. 276: 3756-63.

Dowhan W. 1997. Molecular basis for membrane phospholipid diversity: why are there so many lipids? Ann. Rev. Biochem. 66: 199-232.

Dowhan W, Mileykovskaya E, Bogdanov M. 2004. Diversity and versatility of lipid-protein interactions revealed by molecular genetic approaches. Biochim. Biophys. Acta 1666: 19-39.

Dunn WA, Cregg JM, Kiel JA, van Der Klei IJ, Oku M, Sakai Y, Sibirny AA, Stasyk OV, Veenhuis M. 2005. Pexophagy: the selective autophagy of peroxisomes. Autophagy 1: 75-83. 
Duronio RJ, Knoll LJ, Gordon JI. 1992. Isolation of a Saccharomyces cerevisiae long chain fatty acyl:CoA synthetase gene (FAA1) and assessment of its role in protein N-myristoylation. J. Cell Biol. 117: 515-29.

Dwivedi M, Ahnn J. 2009. Autophagy--is it a preferred route for lifespan extension? BMB Rep. 42: 62-71.

Eide DJ. 2003. Multiple regulatory mechanisms maintain zinc homeostasis in Saccharomyces cerevisiae. J. Nutr. 133: 1532S-5S.

Eisenhaber B, Maurer-Stroh S, Novatchkova M, Schneider G, Eisenhaber F. 2003. Enzymes and auxiliary factors for GPI lipid anchor biosynthesis and post-translational transfer to proteins. BioEssays 25: 367-85.

Epple UD, Eskelinen E, Thumm M. 2003. Intravacuolar membrane lysis in Saccharomyces cerevisiae. Does vacuolar targeting of Cvt17/Aut5p affect its function? J. Biol. Chem. 278: 7810-21.

Epple UD, Suriapranata I, Eskelinen EL, Thumm M. 2001. Aut5/Cvt17p, a putative lipase essential for disintegration of autophagic bodies inside the vacuole. J. Bacteriol. 183: 5942-55.

Faergeman NJ, Knudsen J. 1997. Role of long-chain fatty acyl-CoA esters in the regulation of metabolism and in cell signalling. Biochem. J. 323: 1-12.

Faergeman NJ, Black PN, Zhao XD, Knudsen J, DiRusso CC. 2001. The acyl-CoA synthetases encoded within FAA1 and FAA4 in Saccharomyces cerevisiae function as components of the fatty acid transport system linking import, activation, and intracellular utilization. J. Biol. Chem. 276: 37051-9.

Faergeman NJ, DiRusso CC, Elberger A, Knudsen J, Black PN. 1997. Disruption of the Saccharomyces cerevisiae homologue to the murine fatty acid transport protein impairs uptake and growth on long-chain fatty acids. J. Biol. Chem. 272: 8531-8.

Farré J, Subramani S. 2004. Peroxisome turnover by micropexophagy: an autophagy-related process. Trends Cell Biol. 14: 515-23.

Faulkner A, Chen X, Rush J, Horazdovsky B, Waechter CJ, Carman GM, Sternweis PC. 1999. The LPP1 and DPP1 gene products account for most of the isoprenoid phosphate phosphatase activities in Saccharomyces cerevisiae. J. Biol. Chem. 274: 14831-7.

Fernández-Murray JP, McMaster CR. 2005. Glycerophosphocholine catabolism as a new route for choline formation for phosphatidylcholine synthesis by the Kennedy pathway. J. Biol. Chem 280: 38290-6.

Fernández-Murray JP, McMaster CR. 2007. Phosphatidylcholine synthesis and its catabolism by yeast neuropathy target esterase 1. Biochim. Biophys. Acta 1771: 331-6.

Fischl AS, Carman GM. 1983. Phosphatidylinositol biosynthesis in Saccharomyces cerevisiae: purification and properties of microsome-associated phosphatidylinositol synthase. J. Bacteriol. 154: 304-11.

Flanagan C, Schnieders E, Emerick A, Kunisawa R, Admon A, Thorner J. 1993. Phosphatidylinositol 4kinase: gene structure and requirement for yeast cell viability. Science 262: 1444-1448.

Flick JS, Thorner J. 1993. Genetic and biochemical characterization of a phosphatidylinositol-specific phospholipase C in Saccharomyces cerevisiae. Mol. Cell Biol. 13: 5861-76.

Folch J, Lees M, Sloane Stanley GH. 1957. A simple method for the isolation and purification of total lipides from animal tissues. J. Biol. Chem 226: 497-509.

Fujita M, Umemura M, Yoko-o T, Jigami Y. 2006. PER1 is required for GPI-phospholipase A2 activity and involved in lipid remodeling of GPI-anchored proteins. Mol. Biol. Cell 17: 5253-64. 
Furneisen JM, Carman GM. 2000. Enzymological properties of the LPP1-encoded lipid phosphatase from Saccharomyces cerevisiae. Biochim. Biophys. Acta 1484: 71-82.

Fyrst H, Oskouian B, Kuypers FA, Saba JD. 1999. The PLB2 gene of Saccharomyces cerevisiae confers resistance to lysophosphatidylcholine and encodes a phospholipase $\mathrm{B} /$ lysophospholipase. Biochemistry $\mathbf{3 8}$ : 5864-5871.

Gaigg B, Simbeni R, Hrastnik C, Paltauf F, Daum G. 1995. Characterization of a microsomal subfraction associated with mitochondria of the yeast, Saccharomyces cerevisiae. Involvement in synthesis and import of phospholipids into mitochondria. Biochim. Biophys. Acta 1234: 214-20.

Garbarino J, Sturley SL. 2009. Saturated with fat: new perspectives on lipotoxicity. Curr. Opin. Clin. Nutr. 12: 110-6.

Garcia-Bustos JF, Marini F, Stevenson I, Frei C, Hall MN. 1994. PIK1, an essential phosphatidylinositol 4kinase associated with the yeast nucleus. EMBO J. 13: 2352-61.

Gardenour KR, Levy J, Lopes JM. 2004. Identification of novel dominant INO2c mutants with an Opiphenotype. Mol. Microbiol. 52: 1271-80.

Gasch AP, Spellman PT, Kao CM, Carmel-Harel O, Eisen MB, Storz G, Botstein D, Brown PO. 2000. Genomic expression programs in the response of yeast cells to environmental changes. Mol. Biol. Cell 11: 4241-57.

Gaspar ML, Aregullin MA, Jesch SA, Nunez LR, Villa-García M, Henry SA. 2007. The emergence of yeast lipidomics. Biochim. Biophys. Acta 1771: 241-54.

Gavin A, Bösche M, Krause R, Grandi P, Marzioch M, Bauer A, Schultz J, Rick JM, Michon A, Cruciat C, Remor M, Höfert C, Schelder M, Brajenovic M, Ruffner H, Merino A, Klein K, Hudak M, Dickson D, Rudi T, Gnau V, Bauch A, Bastuck S, Huhse B, Leutwein C, Heurtier M, Copley RR, Edelmann A, Querfurth E, Rybin V, Drewes G, Raida M, Bouwmeester T, Bork P, Seraphin B, Kuster B, Neubauer G, Superti-Furga G. 2002. Functional organization of the yeast proteome by systematic analysis of protein complexes. Nature 415: 141-7.

Gaynor PM, Hubbell S, Schmidt AJ, Lina RA, Minskoff SA, Greenberg ML. 1991. Regulation of phosphatidylglycerolphosphate synthase in Saccharomyces cerevisiae by factors affecting mitochondrial development. J. Bacteriol. 173: 6124-31.

George MD, Baba M, Scott SV, Mizushima N, Garrison BS, Ohsumi Y, Klionsky DJ. 2000. Apg5p functions in the sequestration step in the cytoplasm-to-vacuole targeting and macroautophagy pathways. Mol. Biol. Cell 11: 969-82.

Ghosh AK, Ramakrishnan G, Chandramohan C, Rajasekharan R. 2008b. CGI-58, the causative gene for Chanarin-Dorfman syndrome, mediates acylation of lysophosphatidic acid. J. Biol. Chem. 283: 24525-33.

Ghosh AK, Ramakrishnan G, Rajasekharan R. 2008a. YLRO99C (ICT1) encodes a soluble acyl-CoAdependent lysophosphatidic acid acyltransferase responsible for enhanced phospholipid synthesis on organic solvent stress in Saccharomyces cerevisiae. J. Biol. Chem. 283: 9768-75.

Gietz D, St Jean A, Woods RA, Schiestl RH. 1992. Improved method for high efficiency transformation of intact yeast cells. Nucleic Acids Res. 20: 1425.

Goldstein AL, McCusker JH. 1999. Three new dominant drug resistance cassettes for gene disruption in Saccharomyces cerevisiae. Yeast 15: 1541-53. 
Graves JA, Henry SA. 2000. Regulation of the yeast INO1 gene. The products of the INO2, INO4 and OPII regulatory genes are not required for repression in response to inositol. Genetics 154: 1485-95.

Gray JV, Petsko GA, Johnston GC, Ringe D, Singer RA, Werner-Washburne M. 2004. "Sleeping beauty": quiescence in Saccharomyces cerevisiae. Microbiol. Mol. Biol. Rev. 68: 187-206.

Greenberg ML, Hubbell S, Lam C. 1988. Inositol regulates phosphatidylglycerolphosphate synthase expression in Saccharomyces cerevisiae. Mol. Cell Biol. 8: 4773-9.

Gu Z, Valianpour F, Chen S, Vaz FM, Hakkaart GA, Wanders RJ, Greenberg ML. 2004. Aberrant cardiolipin metabolism in the yeast taz1 mutant: a model for Barth syndrome. Mol. Microbiol. 51: 149-158.

Güldener U, Heck S, Fiedler T, Beinhauer J, Hegemann JH. 1996. A new efficient gene disruption cassette for repeated use in budding yeast. Nucleic Acids Res. 24: 2519-2524.

Güldener U, Heinisch J, Koehler GJ, Voss D, Hegemann JH. 2002. A second set of loxP marker cassettes for Cre-mediated multiple gene knockouts in budding yeast. Nucleic Acids Res. 30: 1-8.

Habeler G, Natter K, Thallinger GG, Crawford ME, Kohlwein SD, Trajanoski Z. 2002. YPL.db: the Yeast Protein Localization database. Nucleic Acids Res. 30: 80-3.

Ham HJ, Rho HJ, Shin SK, Yoon H. 2009. The TGL2 gene of Saccharomyces cerevisiae encodes an active acylglycerol lipase located in the mitochondria. J. Biol. Chem. In Press.

Hamilton JA. 1998. Fatty acid transport: difficult or easy? J. Lipid Res. 39: 467-81.

Hamilton JA, Kamp F. 1999. How are free fatty acids transported in membranes? Is it by proteins or by free diffusion through the lipids? Diabetes 48: 2255-69.

Han G, Gable K, Kohlwein SD, Beaudoin F, Napier JA, Dunn TM. 2002. The Saccharomyces cerevisiae YBR159w gene encodes the 3-ketoreductase of the microsomal fatty acid elongase. J. Biol. Chem. 277: 35440-9.

Han S, Han G, Iwanyshyn WM, Carman GM. 2005. Regulation of the PIS1-encoded phosphatidylinositol synthase in Saccharomyces cerevisiae by zinc. J. Biol. Chem. 280: 29017-24.

Han GS, Johnston CN, Chen X, Athenstaedt K, Daum G, Carman GM. 2001. Regulation of the Saccharomyces cerevisiae DPP1-encoded diacylglycerol pyrophosphate phosphatase by zinc. J. Biol. Chem. 276: 10126-33.

Han G, Johnston CN, Carman GM. 2004. Vacuole membrane topography of the DPP1-encoded diacylglycerol pyrophosphate phosphatase catalytic site from Saccharomyces cerevisiae. J. Biol. Chem. 279: 5338-45.

Han G, O'Hara L, Carman GM, Siniossoglou S. 2008b. An unconventional diacylglycerol kinase that regulates phospholipid synthesis and nuclear membrane growth. J. Biol. Chem. 283: 20433-42.

Han G, O'Hara L, Siniossoglou S, Carman GM. 2008a. Characterization of the yeast DGK1-encoded CTPdependent diacylglycerol kinase. J. Biol. Chem. 283: 20443-53.

Han G, Wu W, Carman GM. 2006. The Saccharomyces cerevisiae Lipin homolog is a $\mathrm{Mg}^{2+}$-dependent phosphatidate phosphatase enzyme. J. Biol. Chem. 281: 9210-8.

Harding TM, Hefner-Gravink A, Thumm M, Klionsky DJ. 1996. Genetic and phenotypic overlap between autophagy and the cytoplasm to vacuole protein targeting pathway. J. Biol. Chem. 271: 17621-4. 
Harding TM, Morano KA, Scott SV, Klionsky DJ. 1995. Isolation and characterization of yeast mutants in the cytoplasm to vacuole protein targeting pathway. J. Cell Biol. 131: 591-602.

Harington A, Herbert CJ, Tung B, Getz GS, Slonimski PP. 1993. Identification of a new nuclear gene (CEM1) encoding a protein homologous to a beta-keto-acyl synthase which is essential for mitochondrial respiration in Saccharomyces cerevisiae. Mol. Microbiol. 9: 545-55.

Hasslacher M, Ivessa AS, Paltauf F, Kohlwein SD. 1993. Acetyl-CoA carboxylase from yeast is an essential enzyme and is regulated by factors that control phospholipid metabolism. J. Biol. Chem. 268: 10946-52.

Hayward AP, Tsao J, Dinesh-Kumar SP. 2009. Autophagy and plant innate immunity: Defense through degradation. Semin.Cel Dev.Biol. 20: 1041-7.

He Q, Greenberg ML. 2004. Post-translational regulation of phosphatidylglycerolphosphate synthase in response to inositol. Mol. Microbiol. 53: 1243-9.

He C, Klionsky DJ. 2009. Regulation mechanisms and signaling pathways of autophagy. Annu. Rev. Genet. 43: 67-93.

Henry SA, Patton-Vogt JL. 1998. Genetic regulation of phospholipid metabolism: yeast as a model eukaryote. Prog. Nucleic Acid Res. Mol. Biol. 61: 133-79.

Hettema EH, Tabak HF. 2000. Transport of fatty acids and metabolites across the peroxisomal membrane. Biochim. Biophys. Acta 1486: 18-27.

Hettema EH, Girzalsky W, van Den Berg M, Erdmann R, Distel B. 2000. Saccharomyces cerevisiae Pex3p and Pex19p are required for proper localization and stability of peroxisomal membrane proteins. EMBO J. 19: 223-33.

Hettema EH, van Roermund CW, Distel B, van Den Berg M, Vilela C, Rodrigues-Pousada C, Wanders RJ, Tabak HF. 1996. The $A B C$ transporter proteins Pat1 and Pat2 are required for import of long-chain fatty acids into peroxisomes of Saccharomyces cerevisiae. EMBO J. 15: 3813-22.

Heyken W, Repenning A, Kumme J, Schüller H. 2005. Constitutive expression of yeast phospholipid biosynthetic genes by variants of Ino2 activator defective for interaction with Opi1 repressor. Mol. Microbiol. 56: 696-707.

Hiltunen JK, Mursula AM, Rottensteiner H, Wierenga RK, Kastaniotis AJ, Gurvitz A. 2003. The biochemistry of peroxisomal beta-oxidation in the yeast Saccharomyces cerevisiae. FEMS Microbiol. Rev. 27: 35-64.

Hiltunen JK, Okubo F, Kursu VA, Autio KJ, Kastaniotis AJ. 2005. Mitochondrial fatty acid synthesis and maintenance of respiratory competent mitochondria in yeast. Biochem. Soc. Trans. 33: 1162-5.

Hjelmstad RH, Bell RM. 1987. Mutants of Saccharomyces cerevisiae defective in sn-1,2-diacylglycerol cholinephosphotransferase. Isolation, characterization, and cloning of the CPT1 gene. J. Biol. Chem. 262: 3909-17.

Hjelmstad RH, Bell RM. 1988. The sn-1,2-diacylglycerol ethanolaminephosphotransferase activity of Saccharomyces cerevisiae. Isolation of mutants and cloning of the EPT1 gene. J. Biol. Chem. 263: 19748-57.

Hoja U, Marthol S, Hofmann J, Stegner S, Schulz R, Meier S, Greiner E, Schweizer E. 2004. HFA1 encoding an organelle-specific acetyl-CoA carboxylase controls mitochondrial fatty acid synthesis in Saccharomyces cerevisiae. J. Biol. Chem. 279: 21779-86. 
Holland KM, Homann MJ, Belunis CJ, Carman GM. 1988. Regulation of phosphatidylinositol kinase activity in Saccharomyces cerevisiae. J. Bacteriol. 170: 828-33.

Hosaka K, Yamashita S. 1984. Regulatory role of phosphatidate phosphatase in triacylglycerol synthesis of Saccharomyces cerevisiae. Biochim. Biophys. Acta 796: 110-7.

Hosaka K, Kodaki T, Yamashita S. 1989. Cloning and characterization of the yeast CKI gene encoding choline kinase and its expression in Escherichia coli. J. Biol. Chem. 264: 2053-9.

Huh W, Falvo JV, Gerke LC, Carroll AS, Howson RW, Weissman JS, O'Shea EK. 2003. Global analysis of protein localization in budding yeast. Nature 425: 686-91.

Huisinga KL, Pugh BF. 2004. A genome-wide housekeeping role for TFIID and a highly regulated stressrelated role for SAGA in Saccharomyces cerevisiae. Mol. Cell 13: 573-85.

Ito H, Fukuda Y, Murata K, Kimura A. 1983. Transformation of intact yeast cells treated with alkali cations. J. Bacteriol. 153: 163-8.

Iwanyshyn WM, Han G, Carman GM. 2004. Regulation of phospholipid synthesis in Saccharomyces cerevisiae by zinc. J. Biol. Chem. 279: 21976-83.

Iwata A, Christianson JC, Bucci M, Ellerby LM, Nukina N, Forno LS, Kopito RR. 2005. Increased susceptibility of cytoplasmic over nuclear polyglutamine aggregates to autophagic degradation. Proc. Natl. Acad. Sci. U.S.A. 102: 13135-40.

Jain S, Stanford N, Bhagwat N, Seiler B, Costanzo M, Boone C, Oelkers P. 2007. Identification of a novel lysophospholipid acyltransferase in Saccharomyces cerevisiae. J. Biol. Chem. 282: 30562-9.

Jandrositz A, Petschnigg J, Zimmermann R, Natter K, Scholze H, Hermetter A, Kohlwein SD, Leber R. 2005. The lipid droplet enzyme Tgl1p hydrolyzes both steryl esters and triglycerides in the yeast, Saccharomyces cerevisiae. Biochim. Biophys. Acta 1735: 50-8.

Janitor M, Subík J. 1993. Molecular cloning of the PEL1 gene of Saccharomyces cerevisiae that is essential for the viability of petite mutants. Curr. Genet. 24: 307-12.

Jensen-Pergakes K, Guo Z, Giattina M, Sturley SL, Bard M. 2001. Transcriptional regulation of the two sterol esterification genes in the yeast Saccharomyces cerevisiae. J. Bacteriol. 183: 4950-7.

Jessen D. 2008. Lipid remodelling in mutants of Saccharomyces cerevisiae. M.Sc. thesis, Georg-August University Göttingen.

Jiang F, Rizavi HS, Greenberg ML. 1997. Cardiolipin is not essential for the growth of Saccharomyces cerevisiae on fermentable or non-fermentable carbon sources. Mol. Microbiol. 26: 481-91.

Johnson DR, Knoll LJ, Levin DE, Gordon JI. 1994a. Saccharomyces cerevisiae contains four fatty acid activation (FAA) genes: an assessment of their role in regulating protein $\mathrm{N}$-myristoylation and cellular lipid metabolism. J. Cell Biol. 127: 751-62.

Johnson DR, Knoll LJ, Rowley N, Gordon JI. 1994b. Genetic Analysis of the Role of Saccharomyces cerevisiae Acyl-CoA Synthetase Genes in Regulating Protein N-Myristoylation J. Biol. Chem. 269: 1803746.

Jones CL, Hajra AK. 1983. Solubilization and partial purification of dihydroxyacetone-phosphate acyltransferase from guinea pig liver. Arch. Biochem. Biophys. 226: 155-65. 
Jones JM, Nau K, Geraghty MT, Erdmann R, Gould SJ. 1999. Identification of peroxisomal acyl-CoA thioesterases in yeast and humans. J. Biol. Chem. 274: 9216-23.

Kaadige MR, Lopes JM. 2006. Analysis of Opi1p repressor mutants. Curr. Genet. 49: 30-8.

Kal AJ, van Zonneveld AJ, Benes V, van Den Berg M, Koerkamp MG, Albermann K, Strack N, Ruijter JM, Richter A, Dujon B, Ansorge W, Tabak HF. 1999. Dynamics of gene expression revealed by comparison of serial analysis of gene expression transcript profiles from yeast grown on two different carbon sources. Mol. Biol. Cell 10: 1859-72.

Kamimoto T, Shoji S, Hidvegi T, Mizushima N, Umebayashi K, Perlmutter DH, Yoshimori T. 2006. Intracellular inclusions containing mutant alpha1-antitrypsin Z are propagated in the absence of autophagic activity. J. Biol. Chem 281: 4467-76.

Kanipes MI, Henry SA. 1997. The phospholipid methyltransferases in yeast. Biochim. Biophys. Acta 1348: 134-41.

Kastaniotis AJ, Autio KJ, Sormunen RT, Hiltunen JK. 2004. Htd2p/Yhr067p is a yeast 3-hydroxyacyl-ACP dehydratase essential for mitochondrial function and morphology. Mol. Microbiol. 53: 1407-21.

Kearsey SE. 1993. Identification of a Saccharomyces cerevisiae gene closely related to FAS3 (acetyl-CoA carboxylase). DNA Seq. 4: 69-70.

Kelley MJ, Carman GM. 1987. Purification and characterization of CDP-diacylglycerol synthase from Saccharomyces cerevisiae. J. Biol. Chem. 262: 14563-70.

Kelley MJ, Bailis AM, Henry SA, Carman GM. 1988. Regulation of phospholipid biosynthesis in Saccharomyces cerevisiae by inositol. Inositol is an inhibitor of phosphatidylserine synthase activity. J. Biol. Chem. 263: 18078-85.

Kennedy EP, Weiss SB. 1956. The function of cytidine coenzymes in the biosynthesis of phospholipides. J. Biol. Chem. 222: 193-214.

Kent C, Carman GM. 1999. Interactions among pathways for phosphatidylcholine metabolism, CTP synthesis and secretion through the Golgi apparatus. Trends Biochem. Sci. 24: 146-150.

Kersting MC, Carman GM. 2006. Regulation of the Saccharomyces cerevisiae EKI1-encoded ethanolamine kinase by zinc depletion. J. Biol. Chem. 281: 13110-6.

Kim J, Dalton VM, Eggerton KP, Scott SV, Klionsky DJ. 1999b. Apg7p/Cvt2p is required for the cytoplasmto-vacuole targeting, macroautophagy, and peroxisome degradation pathways. Mol. Biol. Cell 10: 1337-51.

Kim K, Kim KH, Storey MK, Voelker DR, Carman GM. 1999a. Isolation and characterization of the Saccharomyces cerevisiae EKI1 gene encoding ethanolamine kinase. J. Biol. Chem.274: 14857-66.

Kim I, Rodriguez-Enriquez S, Lemasters JJ. 2007. Selective degradation of mitochondria by mitophagy. Arch. Biochem.Biophys. 462: 245-53.

Kim KH, Voelker DR, Flocco MT, Carman GM. 1998. Expression, purification, and characterization of choline kinase, product of the CKI gene from Saccharomyces cerevisiae. J. Biol. Chem. 273: 6844-52.

Kirkegaard K, Taylor MP, Jackson WT. 2004. Cellular autophagy: surrender, avoidance and subversion by microorganisms. Nat. Rev. Microbiol. 2: 301-14.

Klionsky DJ. 2005. The molecular machinery of autophagy: unanswered questions. J. Cell Sci. 118: 7-18. 
Klionsky DJ, Cuervo AM, Seglen PO. 2007. Methods for monitoring autophagy from yeast to human. Autophagy 3: 181-206.

Knoll LJ, Johnson DR, Gordon JI. 1994. Biochemical studies of three Saccharomyces cerevisiae acyl-CoA synthetases, Faa1p, Faa2p, and Faa3p. J. Biol. Chem. 269: 16348-56.

Knoll LJ, Johnson DR, Gordon JI. 1995. Complementation of Saccharomyces cerevisiae strains containing fatty acid activation gene (FAA) deletions with a mammalian acyl-CoA synthetase. J. Biol. Chem. 270: 10861-7.

Kodaki T, Yamashita S. 1987. Yeast phosphatidylethanolamine methylation pathway. Cloning and characterization of two distinct methyltransferase genes. J. Biol. Chem. 262: 15428-35.

Kodaki T, Yamashita S. 1989. Characterization of the methyltransferases in the yeast phosphatidylethanolamine methylation pathway by selective gene disruption. Eur. J. Biochem. 185: 243-51.

Kohlwein SD, Eder S, Oh CS, Martin CE, Gable K, Bacikova D, Dunn T. 2001. Tsc13p is required for fatty acid elongation and localizes to a novel structure at the nuclear-vacuolar interface in Saccharomyces cerevisiae. Mol. Cell Biol. 21: 109-25.

Koynova R, Caffrey M. 1994. Phases and phase transitions of the hydrated phosphatidylethanolamines. Chem. Phys. Lipids 69: 1-34.

Kraft C, Deplazes A, Sohrmann M, Peter M. 2008. Mature ribosomes are selectively degraded upon starvation by an autophagy pathway requiring the Ubp3p/Bre5p ubiquitin protease. Nat. Cell Biol. 10: 60210.

Kraft C, Reggiori F, Peter M. 2009. Selective types of autophagy in yeast. Biochim. Biophys. Acta 1793: 1404-12.

Krick R, Muehe Y, Prick T, Bremer S, Schlotterhose P, Eskelinen E, Millen J, Goldfarb DS, Thumm M. 2008. Piecemeal microautophagy of the nucleus requires the core macroautophagy genes. Mol. Biol. Cell 19: 4492-505.

Kristjuhan A, Walker J, Suka N, Grunstein M, Roberts D, Cairns BR, Svejstrup JQ. 2002. Transcriptional inhibition of genes with severe histone h3 hypoacetylation in the coding region. Mol. Cell 10: 925-33.

Kruse KB, Brodsky JL, McCracken AA. 2006. Characterization of an ERAD gene as VPS30/ATG6 reveals two alternative and functionally distinct protein quality control pathways: one for soluble $Z$ variant of human alpha-1 proteinase inhibitor (A1PiZ) and another for aggregates of A1PiZ. Mol. Biol. Cell 17: 203-12.

Kuchler K, Daum G, Paltauf F. 1986. Subcellular and submitochondrial localization of phospholipidsynthesizing enzymes in Saccharomyces cerevisiae. J. Bacteriol. 165: 901-10.

Kuge O, Nishijima M. 1997. Phosphatidylserine synthase I and II of mammalian cells. Biochim. Biophys. Acta 1348: 151-156.

Kuma A, Hatano M, Matsui M, Yamamoto A, Nakaya H, Yoshimori T, Ohsumi Y, Tokuhisa T, Mizushima N. 2004. The role of autophagy during the early neonatal starvation period. Nature 432: 1032-6.

Kuma A, Mizushima N, Ishihara N, Ohsumi Y. 2002. Formation of the approximately 350-kDa Apg12Apg5.Apg16 multimeric complex, mediated by Apg16 oligomerization, is essential for autophagy in yeast. J. Biol. Chem. 277: 18619-25. 
Kunau WH, Bühne S, de La Garza M, Kionka C, Mateblowski M, Schultz-Borchard U, Thieringer R. 1988. Comparative enzymology of beta-oxidation. Biochem. Soc. Trans. 16: 418-20.

Kurat CF, Natter K, Petschnigg J, Wolinski H, Scheuringer K, Scholz H, Zimmermann R, Leber R, Zechner R, Kohlwein SD. 2006. Obese yeast: triglyceride lipolysis is functionally conserved from mammals to yeast. $J$. Biol. Chem. 281: 491-500.

Kurat CF, Wolinski H, Petschnigg J, Kaluarachchi S, Andrews B, Natter K, Kohlwein SD. 2009. Cdk1/Cdc28dependent activation of the major triacylglycerol lipase $\mathrm{Tg} \mid 4$ in yeast links lipolysis to cell-cycle progression. Mol. Cell 33: 53-63.

Köffel R, Schneiter R. 2006. Yeh1 constitutes the major steryl ester hydrolase under heme-deficient conditions in Saccharomyces cerevisiae. Eukaryot. Cell 5: 1018-25.

Köffel R, Tiwari R, Falquet L, Schneiter R. 2005. The Saccharomyces cerevisiae YLLO12/YEH1, YLR020/YEH2, and TGL1 genes encode a novel family of membrane-anchored lipases that are required for steryl ester hydrolysis. Mol. Cell Biol. 25: 1655-68.

Lands WE. 1958. Metabolism of glycerolipides; a comparison of lecithin and triglyceride synthesis. J. Biol. Chem. 231: 883-8.

Lands WE. 1960. Metabolism of glycerolipids. J. Biol. Chem. 235: 2233-37.

Lee KS, Patton JL, Fido M, Hines LK, Kohlwein SD, Paltauf F, Henry SA, Levin DE. 1994. The Saccharomyces cerevisiae PLB1 gene encodes a protein required for lysophospholipase and phospholipase B activity. J. Biol. Chem. 269: 19725-19730.

Lehner R, Kuksis A. 1996. Biosynthesis of triacylglycerols. Prog. Lipid Res. 35: 169-201.

Lester RL, Dickson RC. 1993. Sphingolipids with inositolphosphate-containing head groups. Adv. Lipid Res. 26: 253-74.

Letts VA, Klig LS, Bae-Lee M, Carman GM, Henry SA. 1983. Isolation of the yeast structural gene for the membrane-associated enzyme phosphatidylserine synthase. Proc. Natl. Acad. Sci. U.S.A. 80: 7279-83.

Levine B. 2005. Eating oneself and uninvited guests: autophagy-related pathways in cellular defense. Cell 120: $159-62$.

Levine B, Klionsky DJ. 2004. Development by self-digestion: Molecular mechanisms and biological functions of autophagy. Dev. Cell 6: 463-477.

Li X, Gerber SA, Rudner AD, Beausoleil SA, Haas W, Villén J, Elias JE, Gygi SP. 2007. Large-scale phosphorylation analysis of alpha-factor-arrested Saccharomyces cerevisiae. J. Proteome Res. 6: 1190-7.

Listenberger LL, Han X, Lewis SE, Cases S, Farese RV, Ory DS, Schaffer JE. 2003. Triglyceride accumulation protects against fatty acid-induced lipotoxicity. Proc. Natl. Acad. Sci. U.S.A. 100: 3077-82.

Lockshon D, Surface LE, Kerr EO, Kaeberlein M, Kennedy BK. 2007. The sensitivity of yeast mutants to oleic acid implicates the peroxisome and other processes in membrane function. Genetics 175: 77-91.

Loewen CJ, Levine TP. 2005. A highly conserved binding site in vesicle-associated membrane proteinassociated protein (VAP) for the FFAT motif of lipid-binding proteins. J. Biol. Chem. 280: 14097-104.

Loewen CJ, Gaspar ML, Jesch SA, Delon C, Ktistakis NT, Henry SA, Levine TP. 2004. Phospholipid metabolism regulated by a transcription factor sensing phosphatidic acid. Science 304: 1644-7. 
Loewen CJ, Roy A, Levine TP. 2003. A conserved ER targeting motif in three families of lipid binding proteins and in Opi1p binds VAP. EMBO J. 22: 2025-35.

Longo VD, Finch CE. 2003. Evolutionary medicine: from dwarf model systems to healthy centenarians? Science 299: 1342-6.

Lopes JM, Henry SA. 1991. Interaction of trans and cis regulatory elements in the INO1 promoter of Saccharomyces cerevisiae. Nucleic Acids Res. 19: 3987-94.

Lynen F, Hopper-Kessel I, Eggerer H. 1964. On the biosynthesis of fatty acids. 3. Fatty acid synthetase in yeast and the formation of enzyme bound acetoacetic acid. Biochem. Z. 340: 95-124.

MacDiarmid CW, Gaither LA, Eide D. 2000. Zinc transporters that regulate vacuolar zinc storage in Saccharomyces cerevisiae. EMBO J. 19: 2845-55.

Mah AS, Elia AE, Devgan G, Ptacek J, Schutkowski M, Snyder M, Yaffe MB, Deshaies RJ. 2005. Substrate specificity analysis of protein kinase complex Dbf2-Mob1 by peptide library and proteome array screening. BMC Biochem. 6: 22.

Martin CE, Oh C, Jiang Y. 2007. Regulation of long chain unsaturated fatty acid synthesis in yeast. Biochim. Biophys. Acta 1771: 271-85.

Massey A, Kiffin R, Cuervo AM. 2004. Pathophysiology of chaperone-mediated autophagy. Int. J. Biochem. Cell B. 36: 2420-34.

Matsuura A, Tsukada M, Wada Y, Ohshumi Y. 1997. Apg1p, a novel protein kinase required for the autophagic process in Saccharomyces cerevisiae. Gene 192: 245-250.

McDermott M, Wakelam MJ, Morris AJ. 2004. Phospholipase D. Biochem. Cell Biol. 82: 225-53.

McGraw P, Henry SA. 1989. Mutations in the Saccharomyces cerevisiae opi3 gene: effects on phospholipid methylation, growth and cross-pathway regulation of inositol synthesis. Genetics 122: 317-30.

McLaughlin S, Murray D. 2005. Plasma membrane phosphoinositide organization by protein electrostatics. Nature 438: 605-11.

McMaster C, Bell R. 1997. CDP-ethanolamine:1,2-diacylglycerol ethanolaminephosphotransferase. Biochim. Biophys. Acta 1348: 117-123.

Meléndez A, Tallóczy Z, Seaman M, Eskelinen E, Hall DH, Levine B. 2003. Autophagy genes are essential for dauer development and life-span extension in C. elegans. Science 301: 1387-91.

Merkel O, Fido M, Mayr JA, Prüger H, Raab F, Zandonella G, Kohlwein SD, Paltauf F. 1999. Characterization and function in vivo of two novel phospholipases B/lysophospholipases from Saccharomyces cerevisiae. J. Biol. Chem. 274: 28121-7.

Merkel O, Oskolkova OV, Raab F, El-Toukhy R, Paltauf F. 2005a. Regulation of activity in vitro and in vivo of three phospholipases B from Saccharomyces cerevisiae. Biochem. J. 387: 489-96.

Merkel O, Schmid PC, Paltauf F, Schmid HH. 2005b. Presence and potential signaling function of Nacylethanolamines and their phospholipid precursors in the yeast Saccharomyces cerevisiae. Biochim. Biophys. Acta 1734: 215-9. 
Min-Seok R, Kawamata Y, Nakamura H, Ohta A, Takagi M. 1996. Isolation and characterization of ECT1 gene encoding CTP: phosphoethanolamine cytidylyltransferase of Saccharomyces cerevisiae. J. Biochem. 120: $1040-7$.

Minois N, Lagona F, Frajnt M, Vaupel JW. 2009. Plasticity of death rates in stationary phase in Saccharomyces cerevisiae. Aging Cell 8: 36-44.

Mitchell AG, Martin CE. 1995. A novel cytochrome b5-like domain is linked to the carboxyl terminus of the Saccharomyces cerevisiae delta-9 fatty acid desaturase. J. Biol. Chem. 270: 29766-72.

Mizushima N, Noda T, Yoshimori T, Tanaka Y, Ishii T, George MD, Klionsky DJ, Ohsumi M, Ohsumi Y. 1998. A protein conjugation system essential for autophagy. Nature 395: 395-8.

Mizushima N, Yamamoto A, Matsui M, Yoshimori T, Ohsumi Y. 2004. In vivo analysis of autophagy in response to nutrient starvation using transgenic mice expressing a fluorescent autophagosome marker. Mol. Biol. Cell 15: 1101-11.

Mohamed AH, Chirala SS, Mody NH, Huang WY, Wakil SJ. 1988. Primary structure of the multifunctional alpha subunit protein of yeast fatty acid synthase derived from FAS2 gene sequence. J. Biol. Chem. 263: 12315-25.

Mora GE. 2006. The phospholipase B chapter in the metabolic odyssey of a free fatty acid in a yeast cell. M.Sc. thesis, Georg-August University Göttingen.

Morein S, Andersson A, Rilfors L, Lindblom G. 1996. Wild-type Escherichia coli cells regulate the membrane lipid composition in a "window" between gel and non-lamellar structures. J. Biol. Chem. 271: 6801-9.

Morlock KR, Lin YP, Carman GM. 1988. Regulation of phosphatidate phosphatase activity by inositol in Saccharomyces cerevisiae. J. Bacteriol. 170: 3561-6.

Müller O, Sattler T, Flötenmeyer M, Schwarz H, Plattner H, Mayer A. 2000. Autophagic tubes: vacuolar invaginations involved in lateral membrane sorting and inverse vesicle budding. J. Cell Biol. 151: 519-28.

Müllner H, Deutsch G, Leitner E, Ingolic E, Daum G. 2005. YEH2/YLRO20c encodes a novel steryl ester hydrolase of the yeast Saccharomyces cerevisiae. J. Biol. Chem. 280: 13321-8.

Nagiec MM, Wells GB, Lester RL, Dickson RC. 1993. A suppressor gene that enables Saccharomyces cerevisiae to grow without making sphingolipids encodes a protein that resembles an Escherichia coli fatty acyltransferase. J. Biol. Chem. 268: 22156-22163.

Nakagawa I, Amano A, Mizushima N, Yamamoto A, Yamaguchi H, Kamimoto T, Nara A, Funao J, Nakata M, Tsuda K, Hamada S, Yoshimori T. 2004. Autophagy defends cells against invading group A Streptococcus. Science 306: 1037-40.

Nakashima A, Hosaka K, Nikawa J. 1997. Cloning of a human cDNA for CTP-phosphoethanolamine cytidylyltransferase by complementation in vivo of a yeast mutant. J. Biol. Chem. 272: 9567-72.

Nakayama K, Nakamura T, Taniguchi M, Tanaka T. 2002. Irreversible deacylation of plasma membrane phospholipids by the combined action of $\mathrm{Mg}^{2+}$ and a long-chain acyl-CoA synthetase inhibitor in Saccharomyces cerevisiae. J. Biosci. Bioeng. 94: 258-263.

Natter K, Leitner P, Faschinger A, Wolinski H, McCraith S, Fields S, Kohlwein SD. 2005. The spatial organization of lipid synthesis in the yeast Saccharomyces cerevisiae derived from large scale green fluorescent protein tagging and high resolution microscopy. Mol. Cell. Proteomics 4: 662-72. 
Nikawa J, Yamashita S. 1997. Phosphatidylinositol synthase from yeast. Biochim. Biophys. Acta 1348: 1738.

Nikawa J, Kodaki T, Yamashita S. 1987. Primary structure and disruption of the phosphatidylinositol synthase gene of Saccharomyces cerevisiae. J. Biol. Chem. 262: 4876-81.

Nikoloff DM, Henry SA. 1994. Functional characterization of the INO2 gene of Saccharomyces cerevisiae. A positive regulator of phospholipid biosynthesis. J. Biol. Chem. 269: 7402-11.

O'Hara L, Han G, Peak-Chew S, Grimsey N, Carman GM, Siniossoglou S. 2006. Control of phospholipid synthesis by phosphorylation of the yeast lipin Pah1p/Smp2p Mg ${ }^{2+}$-dependent phosphatidate phosphatase. J. Biol. Chem. 281: 34537-48.

Oelkers P, Cromley D, Padamsee M, Billheimer JT, Sturley SL. 2002. The DGA1 gene determines a second triglyceride synthetic pathway in yeast. J. Biol. Chem. 277: 8877-81.

Oelkers P, Tinkelenberg A, Erdeniz N, Cromley D, Billheimer JT, Sturley SL. 2000. A lecithin cholesterol acyltransferase-like gene mediates diacylglycerol esterification in yeast. J. Biol. Chem. 275: 15609-12.

Ogawa M, Sasakawa C. 2006. Bacterial evasion of the autophagic defense system. Curr. Opin. Microbiol. 9: 62-8.

Oh CS, Toke DA, Mandala S, Martin CE. 1997. ELO2 and ELO3, homologues of the Saccharomyces cerevisiae ELO1 gene, function in fatty acid elongation and are required for sphingolipid formation. J. Biol. Chem. 272: 17376-84.

Ostrander DB, O'Brien DJ, Gorman JA, Carman GM. 1998. Effect of CTP synthetase regulation by CTP on phospholipid synthesis in Saccharomyces cerevisiae. J. Biol. Chem. 273: 18992-9001.

$\emptyset$ verbye A, Fengsrud M, Seglen PO. 2007. Proteomic analysis of membrane-associated proteins from rat liver autophagosomes. Autophagy 3: 300-22.

Owen OE, Reichard GA, Patel MS, Boden G. 1979. Energy metabolism in feasting and fasting. Adv. Exp. Med. Biol. 111: 169-88.

Palecek SP, Parikh AS, Huh JH, Kron SJ. 2002. Depression of Saccharomyces cerevisiae invasive growth on non-glucose carbon sources requires the Snf1 kinase. Mol. Microbiol. 45: 453-469.

Paltauf F, Kohlwein S, Henry S. 1992. Regulation and compartmentalization of lipid synthesis in yeast. In EW Jones, JR Pringle, and JR Broach. The molecular and cellular biology of the yeast Saccharomyces: Gene expression 2: 415-500. Cold Spring Harbor, New York: Cold Spring Harbor Laboratory Press.

Patton-Vogt J. 2007. Transport and metabolism of glycerophosphodiesters produced through phospholipid deacylation. Biochim. Biophys. Acta 1771: 337-42.

Patton-Vogt JL, Griac P, Sreenivas A, Bruno V, Dowd S, Swede MJ, Henry SA. 1997. Role of the yeast phosphatidylinositol/phosphatidylcholine transfer protein (Sec14p) in phosphatidylcholine turnover and INO1 regulation. J. Biol. Chem. 272: 20873-83.

Petschnigg J, Wolinski H, Kolb D, Zellnig G, Kurat CF, Natter K, Kohlwein SD. 2009. Good fat, essential cellular requirements for triacylglycerol synthesis to maintain membrane homeostasis in yeast. J. Biol. Chem. 284: 30981-93. 
Polakowski T, Bastl R, Stahl U, Lang C. 1999. Enhanced sterol-acyl transferase activity promotes sterol accumulation in Saccharomyces cerevisiae. Appl. Microbiol. Biot. 53: 30-35.

Ptacek J, Devgan G, Michaud G, Zhu H, Zhu X, Fasolo J, Guo H, Jona G, Breitkreutz A, Sopko R, McCartney RR, Schmidt MC, Rachidi N, Lee S, Mah AS, Meng L, Stark MJ, Stern DF, De Virgilio C, Tyers M, Andrews B, Gerstein M, Schweitzer B, Predki PF, Snyder M. 2005. Global analysis of protein phosphorylation in yeast. Nature 438: 679-84.

Racenis PV, Lai JL, Das AK, Mullick PC, Hajra AK, Greenberg ML. 1992. The acyl dihydroxyacetone phosphate pathway enzymes for glycerolipid biosynthesis are present in the yeast Saccharomyces cerevisiae. J. Bacteriol. 174: 5702-10.

Rajakumari S, Daum G. 2009. Janus-faced enzymes yeast Tgl3p and Tgl5p catalyze lipase and acyltransferase reactions. Mol. Biol. Cell. In press.

Rajakumari S, Grillitsch K, Daum G. 2008. Synthesis and turnover of non-polar lipids in yeast. Prog. Lipid Res. 47: 157-71.

Rathod PK, Leffers NP, Young RD. 1992. Molecular targets of 5-fluoroorotate in the human malaria parasite, Plasmodium falciparum. Antimicrob. Agents Chemother. 36: 704-11.

Rattray JB, Schibeci A, Kidby DK. 1975. Lipids of Yeasts. Bacteriol. Rev. 39: 197-231.

Rebecchi MJ, Pentyala SN. 2000. Structure, function, and control of phosphoinositide-specific phospholipase C. Physiol. Rev. 80: 1291-335.

Richard MG, McMaster CR. 1998. Lysophosphatidylcholine acyltransferase activity in Saccharomyces cerevisiae: regulation by a high-affinity $\mathrm{Zn}^{2+}$ binding site. Lipids 33: 1229-34.

Riekhof WR, Wu J, Jones JL, Voelker DR. 2007. Identification and characterization of the major lysophosphatidylethanolamine acyltransferase in Saccharomyces cerevisiae. J. Biol. Chem. 282: 28344-52.

Rietveld AG, Killian JA, Dowhan W, de Kruijff B. 1993. Polymorphic regulation of membrane phospholipid composition in Escherichia coli. J. Biol. Chem. 268: 12427-33.

Roberts P, Moshitch-Moshkovitz S, Kvam E, O'Toole E, Winey M, Goldfarb DS. 2003. Piecemeal microautophagy of nucleus in Saccharomyces cerevisiae. Mol. Biol. Cell 14: 129-41.

Rössler H, Rieck C, Delong T, Hoja U, Schweizer E. 2003. Functional differentiation and selective inactivation of multiple Saccharomyces cerevisiae genes involved in very-long-chain fatty acid synthesis. Mol. Genet. Genomics 269: 290-8.

Sanchez-Diaz A, Kanemaki M, Marchesi V, Labib K. 2004. Rapid depletion of budding yeast proteins by fusion to a heat-inducible degron. Sci. STKE 2004: PL8.

Sandager L, Gustavsson MH, Ståhl U, Dahlqvist A, Wiberg E, Banas A, Lenman M, Ronne H, Stymne S. 2002. Storage lipid synthesis is non-essential in yeast. J. Biol. Chem. 277: 6478-82.

Santos-Rosa H, Leung J, Grimsey N, Peak-Chew S, Siniossoglou S. 2005. The yeast lipin Smp2 couples phospholipid biosynthesis to nuclear membrane growth. EMBO J. 24: 1931-41.

Sattler T, Mayer A. 2000. Cell-free reconstitution of microautophagic vacuole invagination and vesicle formation. J. Cell Biol. 151: 529-38. 
Schaffer JE, Lodish HF. 1994. Expression cloning and characterization of a novel adipocyte long chain fatty acid transport protein. Cell 79: 427-436.

Scharnewski M. 2005. Analyse der Fettsaeure-aktivierenden Reaktionen in Mutanten von Saccharomyces cerevisiae. Diplomarbeit, Georg-August Universität Göttingen.

Scharnewski M, Pongdontri P, Mora G, Hoppert M, Fulda M. 2008. Mutants of Saccharomyces cerevisiae deficient in acyl-CoA synthetases secrete fatty acids due to interrupted fatty acid recycling. FEBS J. 275: 2765-78.

Schlossman DM, Bell RM. 1978. Glycerolipid biosynthesis in Saccharomyces cerevisiae: sn-glycerol-3phosphate and dihydroxyacetone phosphate acyltransferase activities. J. Bacteriol. 133: 1368-76.

Schneider R, Brors B, Bürger F, Camrath S, Weiss H. 1997. Two genes of the putative mitochondrial fatty acid synthase in the genome of Saccharomyces cerevisiae. Curr. Genet. 32: 384-8.

Schneider R, Massow M, Lisowsky T, Weiss H. 1995. Different respiratory-defective phenotypes of Neurospora crassa and Saccharomyces cerevisiae after inactivation of the gene encoding the mitochondrial acyl carrier protein. Curr. Genet. 29: 10-7.

Schneiter R, Tatzer V, Gogg G, Leitner E, Kohlwein SD. 2000. Elo1p-dependent carboxy-terminal elongation of $\mathrm{C} 14: 1 \Delta^{9}$ to $\mathrm{C} 16: 1 \Delta^{11}$ fatty acids in Saccharomyces cerevisiae. J. Bacteriol. 182: 3655-60.

Schrag JD, Cygler M. 1997. Lipases and alpha/beta hydrolase fold. Methods Enzymol. 284: 85-107.

Schwank S, Ebbert R, Rautenstrauss K, Schweizer E, Schüller HJ. 1995. Yeast transcriptional activator INO2 interacts as an Ino2p/Ino4p basic helix-loop-helix heteromeric complex with the inositol/cholineresponsive element necessary for expression of phospholipid biosynthetic genes in Saccharomyces cerevisiae. Nucleic Acids Res. 23: 230-7.

Schweizer E, Bolling H. 1970. A Saccharomyces cerevisiae mutant defective in saturated fatty acid biosynthesis. Proc. Natl. Acad. Sci. U.S.A. 67: 660-6.

Schweizer M, Lebert C, Höltke J, Roberts LM, Schweizer E. 1984. Molecular cloning of the yeast fatty acid synthetase genes, FAS1 and FAS2: illustrating the structure of the FAS1 cluster gene by transcript mapping and transformation studies. Mol. Gen. Genet. 194: 457-65.

Schweizer E, Lerch I, Kroeplin-Rueff L, Lynen F. 1970. Fatty acyl transferase. Characterization of the enzyme as part of the yeast fatty acid synthetase complex by the use of radioactively labeled coenzyme $A$. Eur. J. Biochem. 15: 472-482.

Schweizer M, Roberts LM, Höltke H, Takabayashi K, Höllerer E, Hoffmann B, Müller G, Köttig H, Schweizer E. 1986. The pentafunctional FAS1 gene of yeast: its nucleotide sequence and order of the catalytic domains. Mol. Gen. Genet. 203: 479-486.

Schüller HJ, Hahn A, Tröster F, Schütz A, Schweizer E. 1992. Coordinate genetic control of yeast fatty acid synthase genes FAS1 and FAS2 by an upstream activation site common to genes involved in membrane lipid biosynthesis. EMBO J. 11: 107-14.

Scott SV, Baba M, Ohsumi Y, Klionsky DJ. 1997. Aminopeptidase I is targeted to the vacuole by a nonclassical vesicular mechanism. J. Cell Biol. 138: 37-44.

Scott SV, Hefner-Gravink A, Morano KA, Noda T, Ohsumi Y, Klionsky DJ. 1996. Cytoplasm-to-vacuole targeting and autophagy employ the same machinery to deliver proteins to the yeast vacuole. Proc. Natl. Acad. Sci. U.S.A. 93: 12304-8. 
Shani N, Valle D. 1996. A Saccharomyces cerevisiae homolog of the human adrenoleukodystrophy transporter is a heterodimer of two half ATP-binding cassette transporters. Proc. Natl. Acad. Sci. U.S.A. 93: 11901-6.

Shen H, Heacock PN, Clancey CJ, Dowhan W. 1996. The CDS1 gene encoding CDP-diacylglycerol synthase in Saccharomyces cerevisiae is essential for cell growth. J. Biol. Chem. 271: 789-95.

Shintani T, Klionsky DJ. 2004. Autophagy in health and disease: a double-edged sword. Science 306: 990-5.

Shirra MK, Patton-Vogt J, Ulrich A, Liuta-Tehlivets O, Kohlwein SD, Henry SA, Arndt KM. 2001. Inhibition of acetyl coenzyme A carboxylase activity restores expression of the INO1 gene in a snf1 mutant strain of Saccharomyces cerevisiae. Mol. Cell Biol. 21: 5710-22.

Sickmann A, Reinders J, Wagner Y, Joppich C, Zahedi R, Meyer HE, Schönfisch B, Perschil I, Chacinska A, Guiard B, Rehling P, Pfanner N, Meisinger C. 2003. The proteome of Saccharomyces cerevisiae mitochondria. Proc. Natl. Acad. Sci. U.S.A. 100: 13207-12.

Simocková M, Holic R, Tahotná D, Patton-Vogt J, Griac P. 2008. Yeast Pgc1p (YPL206c) controls the amount of phosphatidylglycerol via a phospholipase C-type degradation mechanism. J. Biol. Chem. 283: 17107-15.

Singh R, Kaushik S, Wang Y, Xiang Y, Novak I, Komatsu M, Tanaka K, Cuervo AM, Czaja MJ. 2009. Autophagy regulates lipid metabolism. Nature 458: 1131-5.

Smith JJ, Marelli M, Christmas RH, Vizeacoumar FJ, Dilworth DJ, Ideker T, Galitski T, Dimitrov K, Rachubinski RA, Aitchison JD. 2002. Transcriptome profiling to identify genes involved in peroxisome assembly and function. J. Cell Biol. 158: 259-71.

Sorger D, Daum G. 2002. Synthesis of triacylglycerols by the acyl-coenzyme A:diacyl-glycerol acyltransferase Dga1p in lipid particles of the yeast Saccharomyces cerevisiae. J. Bacteriol. 184: 519-24.

Sorger D, Athenstaedt K, Hrastnik C, Daum G. 2004. A yeast strain lacking lipid particles bears a defect in ergosterol formation. J. Biol. Chem. 279: 31190-6.

Soto A, Carman GM. 2008. Regulation of the Saccharomyces cerevisiae CKI1-encoded choline kinase by zinc depletion. J. Biol. Chem. 283: 10079-88.

Spellman PT, Sherlock G, Zhang MQ, lyer VR, Anders K, Eisen MB, Brown PO, Botstein D, Futcher B. 1998. Comprehensive identification of cell cycle-regulated genes of the yeast Saccharomyces cerevisiae by microarray hybridization. Mol. Biol. Cell 9: 3273-97.

Sreenivas A, Carman GM. 2003. Phosphorylation of the yeast phospholipid synthesis regulatory protein Opi1p by protein kinase A. J. Biol. Chem. 278: 20673-80.

Sreenivas A, Patton-Vogt JL, Bruno V, Griac P, Henry SA. 1998. A role for phospholipase D (Pld1p) in growth, secretion, and regulation of membrane lipid synthesis in yeast. J. Biol. Chem. 273: 16635-8.

Sreenivas A, Villa-Garcia MJ, Henry SA, Carman GM. 2001. Phosphorylation of the yeast phospholipid synthesis regulatory protein Opi1p by protein kinase C. J. Biol. Chem. 276: 29915-23.

Steiner MR, Lester RL. 1970. In vitro study of the methylation pathway of phosphatidylcholine synthesis and the regulation of this pathway in Saccharomyces cerevisiae. Biochemistry 9: 63-9.

Steiner MR, Lester RL. 1972a. In vitro studies of phospholipid biosynthesis in Saccharomyces cerevisiae. Biochim. Biophys. Acta 260: 222-43. 
Steiner S, Lester RL. 1972b. Metabolism of diphosphoinositide and triphosphoinositide in Saccharomyces cerevisiae. Biochim. Biophys. Acta 260: 82-7.

Storey MK, Clay KL, Kutateladze T, Murphy RC, Overduin M, Voelker DR. 2001. Phosphatidylethanolamine has an essential role in Saccharomyces cerevisiae that is independent of its ability to form hexagonal phase structures. J. Biol. Chem. 276: 48539-48.

Straub M, Bredschneider M, Thumm M. 1997. AUT3, a serine/threonine kinase gene, is essential for autophagocytosis in Saccharomyces cerevisiae. J. Bacteriol. 179: 3875-83.

Stuible HP, Meier S, Wagner C, Hannappel E, Schweizer E. 1998. A novel phosphopantetheine:protein transferase activating yeast mitochondrial acyl carrier protein. J. Biol. Chem. 273: 22334-9.

Stukey JE, McDonough VM, Martin CE. 1989. Isolation and characterization of OLE1, a gene affecting fatty acid desaturation from Saccharomyces cerevisiae. J. Biol. Chem. 264: 16537-44.

Stukey JE, McDonough VM, Martin CE. 1990. The OLE1 gene of Saccharomyces cerevisiae encodes the delta 9 fatty acid desaturase and can be functionally replaced by the rat stearoyl-CoA desaturase gene. J. Biol. Chem. 265: 20144-9.

Stålberg K, Neal AC, Ronne H, Ståhl U. 2008. Identification of a novel GPCAT activity and a new pathway for phosphatidylcholine biosynthesis in S. cerevisiae. J. Lipid Res. 49: 1794-806.

Summers EF, Letts VA, McGraw P, Henry SA. 1988. Saccharomyces cerevisiae cho2 mutants are deficient in phospholipid methylation and cross-pathway regulation of inositol synthesis. Genetics 120: 909-22.

Suzuki K, Ohsumi Y. 2007. Molecular machinery of autophagosome formation in yeast, Saccharomyces cerevisiae. FEBS Lett. 581: 2156-61.

Suzuki K, Kubota Y, Sekito T, Ohsumi Y. 2007. Hierarchy of Atg proteins in pre-autophagosomal structure organization. Genes Cells 12: 209-18.

Swartzman EE, Viswanathan MN, Thorner J. 1996. The PAL1 gene product is a peroxisomal ATP-binding cassette transporter in the yeast Saccharomyces cerevisiae. J. Cell Biol. 132: 549-63.

Tamaki H, Shimada A, Ito Y, Ohya M, Takase J, Miyashita M, Miyagawa H, Nozaki H, Nakayama R, Kumagai H. 2007. LPT1 encodes a membrane-bound O-acyltransferase involved in the acylation of lysophospholipids in the yeast Saccharomyces cerevisiae. J. Biol. Chem. 282: 34288-98.

Tanaka K, Fukuda R, Ono Y, Eguchi H, Nagasawa S, Nakatani Y, Watanabe H, Nakanishi H, Taguchi R, Ohta A. 2008. Incorporation and remodeling of extracellular phosphatidylcholine with short acyl residues in Saccharomyces cerevisiae. Biochim. Biophys. Acta 1781: 391-9.

Tang F, Watkins JW, Bermudez M, Gray R, Gaban A, Portie K, Grace S, Kleve M, Craciun G. 2008. A lifespan extending form of autophagy employs the vacuole-vacuole fusion machinery. Autophagy 4: 874-86.

Tanida I, Mizushima N, Kiyooka M, Ohsumi M, Ueno T, Ohsumi Y, Kominami E. 1999. Apg7p/Cvt2p: A novel protein-activating enzyme essential for autophagy. Mol. Biol. Cell 10: 1367-79.

Taylor EB, Ellingson WJ, Lamb JD, Chesser DG, Winder WW. 2005. Long-chain acyl-CoA esters inhibit phosphorylation of AMP-activated protein kinase at threonine-172 by LKB1/STRAD/MO25. Am. J. Physiol. Endocrinol. Metab. 288: E1055-61.

Tehlivets O, Scheuringer K, Kohlwein SD. 2007. Fatty acid synthesis and elongation in yeast. Biochim. Biophys. Acta 1771: 255-70. 
Testet E, Laroche-Traineau J, Noubhani A, Coulon D, Bunoust O, Camougrand N, Manon S, Lessire R, Bessoule J. 2005. Ypr140wp, 'the yeast tafazzin', displays a mitochondrial lysophosphatidylcholine (lyso-PC) acyltransferase activity related to triacylglycerol and mitochondrial lipid synthesis. Biochem. J. 387: 617-26.

Teter SA, Eggerton KP, Scott SV, Kim J, Fischer AM, Klionsky DJ. 2001. Degradation of lipid vesicles in the yeast vacuole requires function of Cvt17, a putative lipase. J. Biol. Chem. 276: 2083-7.

Thomas B, Rothstein R. 1989. Elevated recombination rates in transcriptionally active DNA. Cell 56: 619630.

Thoms S, Debelyy MO, Nau K, Meyer HE, Erdmann R. 2008. Lpx1p is a peroxisomal lipase required for normal peroxisome morphology. FEBS J. 275: 504-14.

Toke DA, Martin CE. 1996. Isolation and characterization of a gene affecting fatty acid elongation in Saccharomyces cerevisiae. J. Biol. Chem. 271: 18413-22.

Toke DA, Bennett WL, Dillon DA, Wu WI, Chen X, Ostrander DB, Oshiro J, Cremesti A, Voelker DR, FischI AS, Carman GM. 1998a. Isolation and characterization of the Saccharomyces cerevisiae DPP1 gene encoding diacylglycerol pyrophosphate phosphatase. J. Biol. Chem. 273: 3278-84.

Toke DA, Bennett WL, Oshiro J, Wu WI, Voelker DR, Carman GM. 1998b. Isolation and characterization of the Saccharomyces cerevisiae LPP1 gene encoding a $\mathrm{Mg}^{2+}$-independent phosphatidate phosphatase. J. Biol. Chem. 273: 14331-8.

Torkko JM, Koivuranta KT, Miinalainen IJ, Yagi Al, Schmitz W, Kastaniotis AJ, Airenne TT, Gurvitz A, Hiltunen KJ. 2001. Candida tropicalis Etr1p and Saccharomyces cerevisiae Ybr026p (Mrf1'p), 2-enoyl thioester reductases essential for mitochondrial respiratory competence. Mol. Cell Biol. 21: 6243-53.

Trotter PJ, Voelker DR. 1995. Identification of a non-mitochondrial phosphatidylserine decarboxylase activity (PSD2) in the yeast Saccharomyces cerevisiae. J. Biol. Chem. 270: 6062-70.

Tsukada M, Ohsumi Y. 1993. Isolation and characterization of autophagy-defective mutants of Saccharomyces cerevisiae. FEBS Lett. 333: 169-74.

Tsukagoshi Y, Nikawa J, Yamashita S. 1987. Molecular cloning and characterization of the gene encoding cholinephosphate cytidylyltransferase in Saccharomyces cerevisiae. Eur. J. Biochem. 169: 477-86.

Tuller G, Hrastnik C, Achleitner G, Schiefthaler U, Klein F, Daum G. 1998. YDL142c encodes cardiolipin synthase (Cls1p) and is non-essential for aerobic growth of Saccharomyces cerevisiae. FEBS Lett. 421: 15-8.

Työrinoja K, Nurminen T, Suomalainen H. 1974. The cell-envelope glycolipids of baker`s yeast. Biochem. J. 141: 133-139.

Ubersax JA, Woodbury EL, Quang PN, Paraz M, Blethrow JD, Shah K, Shokat KM, Morgan DO. 2003. Targets of the cyclin-dependent kinase Cdk1. Nature 425: 859-64.

van Den Brink-van der Laan E, Killian JA, de Kruijff B. 2004. Nonbilayer lipids affect peripheral and integral membrane proteins via changes in the lateral pressure profile. Biochim. Biophys. Acta 1666: 275-88.

van Heusden GP, Nebohâcovâ M, Overbeeke TL, Steensma HY. 1998. The Saccharomyces cerevisiae TGL2 gene encodes a protein with lipolytic activity and can complement an Escherichia coli diacylglycerol kinase disruptant. Yeast 14: 225-32.

van Meer G. 2005. Cellular lipidomics. EMBO J. 24: 3159-65. 
van Roermund CW, Elgersma Y, Singh N, Wanders RJ, Tabak HF. 1995. The membrane of peroxisomes in Saccharomyces cerevisiae is impermeable to $\mathrm{NAD}(\mathrm{H})$ and acetyl-CoA under in vivo conditions. EMBO J. 14: 3480-6.

van Roermund CW, Tabak HF, van Den Berg M, Wanders RJ, Hettema EH. 2000. Pex11p plays a primary role in medium-chain fatty acid oxidation, a process that affects peroxisome number and size in Saccharomyces cerevisiae. J. Cell Biol. 150: 489-98.

van der Klei IJ, Todde V, Veenhuis M. 2009. Autophagy: principles and significance in health and disease. Biochim. Biophys. Acta 1792: 3-13.

Vellai T, Takacs-Vellai K, Zhang Y, Kovacs AL, Orosz L, Müller F. 2003. Genetics: influence of TOR kinase on lifespan in C. elegans. Nature 426: 620.

Voelker DR. 1997. Phosphatidylserine decarboxylase. Biochim. Biophys. Acta 1348: 236-44.

Voss H, Benes V, Andrade MA, Valencia A, Rechmann S, Teodoru C, Schwager C, Paces V, Sander C, Ansorge W. 1997. DNA sequencing and analysis of $130 \mathrm{~kb}$ from yeast chromosome XV. Yeast 13: 655-72.

Wach A, Brachat A, Pöhlmann R, Philippsen P. 1994. New heterologous modules for classical or PCR-based gene disruptions in Saccharomyces cerevisiae. Yeast 10: 1793-808.

Wagner A, Daum G. 2005. Formation and mobilization of neutral lipids in the yeast Saccharomyces cerevisiae. Biochem. Soc. Trans. 33: 1174-7.

Wagner S, Paltauf F. 1994. Generation of glycerophospholipid molecular species in the yeast Saccharomyces cerevisiae. Fatty acid pattern of phospholipid classes and selective acyl turnover at sn-1 and sn-2 positions. Yeast 10: 1429-37.

Wagner C, Dietz M, Wittmann J, Albrecht A, Schüller HJ. 2001. The negative regulator Opi1 of phospholipid biosynthesis in yeast contacts the pleiotropic repressor Sin3 and the transcriptional activator Ino2. Mol. Microbiol. 41: 155-66.

Wagner A, Grillitsch K, Leitner E, Daum G. 2009. Mobilization of steryl esters from lipid particles of the yeast Saccharomyces cerevisiae. Biochim. Biophys. Acta 1791: 118-24.

Waters BM, Eide DJ. 2002. Combinatorial control of yeast FET4 gene expression by iron, zinc, and oxygen. J. Biol. Chem. 277: 33749-57.

Watkins PA, Lu JF, Steinberg SJ, Gould SJ, Smith KD, Braiterman LT. 1998. Disruption of the Saccharomyces cerevisiae FAT1 gene decreases very long-chain fatty acyl-CoA synthetase activity and elevates intracellular very long-chain fatty acid concentrations. J. Biol. Chem. 273: 18210-9.

Welch JW, Burlingame AL. 1973. Very long-chain fatty acids in yeast. J. Bacteriol. 115: 464-6.

Wu WI, Carman GM. 1994. Regulation of phosphatidate phosphatase activity from the yeast Saccharomyces cerevisiae by nucleotides. J. Biol. Chem. 269: 29495-501.

Wu WI, Carman GM. 1996. Regulation of phosphatidate phosphatase activity from the yeast Saccharomyces cerevisiae by phospholipids. Biochemistry 35: 3790-6.

Wu WI, Liu Y, Riedel B, Wissing JB, Fischl AS, Carman GM. 1996. Purification and characterization of diacylglycerol pyrophosphate phosphatase from Saccharomyces cerevisiae. J. Biol. Chem. 271: 1868-76. 
Xie Z, Klionsky DJ. 2007. Autophagosome formation: core machinery and adaptations. Nat. Cell Biol. 9: 1102-9.

Xu Y, Kelley RI, Blanck TJ, Schlame M. 2003. Remodeling of cardiolipin by phospholipid transacylation. J. Biol. Chem. 278: 51380-5.

Xu Y, Malhotra A, Ren M, Schlame M. 2006. The enzymatic function of tafazzin. J. Biol. Chem. 281: 3921724.

Yamada K, Okuyama H, Endo Y, Ikezawa H. 1977. Acyltransferase systems involved in phospholipid metabolism in Saccharomyces cerevisiae. Arch. Biochem. Biophys. 183: 281-9.

Yamashita S, Nikawa J. 1997. Phosphatidylserine synthase from yeast. Biochim. Biophys. Acta 1348: 22835.

Yamashita A, Kawagishi N, Miyashita T, Nagatsuka T, Sugiura T, Kume K, Shimizu T, Waku K. 2001. ATPindependent fatty acyl-coenzyme A synthesis from phospholipid. J. Biol. Chem. 276: 26745-52.

Yamashita A, Sugiura T, Waku K. 1997. Acyltransferases and transacylases involved in fatty acid remodeling of phospholipids and metabolism of bioactive lipids in mammalian cells. J. Biochem. 122: 1-16.

Yamashita A, Watanabe M, Sato K, Miyashita T, Nagatsuka T, Kondo H, Kawagishi N, Nakanishi H, Kamata R, Sugiura T, Waku K. 2003. Reverse reaction of lysophosphatidylinositol acyltransferase. J. Biol. Chem. 278: 30382-93.

Yang H, Bard M, Bruner DA, Gleeson A, Deckelbaum RJ, Aljinovic G, Pohl TM, Rothstein R, Sturley SL. 1996. Sterol esterification in yeast: a two-gene process. Science 272: 1353-6.

Yeagle PL. 1989. Lipid regulation of cell membrane structure and function. FASEB J. 3: 1833-42.

Yeung T, Gilbert GE, Shi J, Silvius J, Kapus A, Grinstein S. 2008. Membrane phosphatidylserine regulates surface charge and protein localization. Science 319: 210-3.

Yoko-o T, Matsui Y, Yagisawa H, Nojima H, Uno I, Toh-e A. 1993. The putative phosphoinositide-specific phospholipase C gene, PLC1, of the yeast Saccharomyces cerevisiae is important for cell growth. Proc. Natl. Acad. Sci. U.S.A. 90: 1804-8.

Yorimitsu T, Klionsky DJ. 2007a. Eating the endoplasmic reticulum: quality control by autophagy. Trends Cell Biol. 17: 279-85.

Yorimitsu T, Klionsky DJ. 2007b. Endoplasmic reticulum stress: a new pathway to induce autophagy. Autophagy 3: 160-2.

Yost RW, Grauvickel SJ, Cantwell R, Bomalaski JS, Hudson AP. 1991. Yeast mitochondria (Saccharomyces cerevisiae) contain $\mathrm{Ca}^{2+}$-independent phospholipase $\mathrm{A} 1$ and $\mathrm{A} 2$ activities: effect of respiratory state. Biochem. Int. 24: 199-208.

Yu C, Kennedy NJ, Chang CC, Rothblatt JA. 1996. Molecular cloning and characterization of two isoforms of Saccharomyces cerevisiae acyl-CoA:sterol acyltransferase. J. Biol. Chem 271: 24157-63.

Zaccheo O, Dinsdale D, Meacock PA, Glynn P. 2004. Neuropathy target esterase and its yeast homologue degrade phosphatidylcholine to glycerophosphocholine in living cells. J. Biol. Chem 279: 24024-33. 
Zaremberg V, McMaster CR. 2002. Differential partitioning of lipids metabolized by separate yeast glycerol-3-phosphate acyltransferases reveals that phospholipase D generation of phosphatidic acid mediates sensitivity to choline-containing lysolipids and drugs. J. Biol. Chem. 277: 39035-44.

Zhao H, Eide D. 1996a. The ZRT2 gene encodes the low affinity zinc transporter in Saccharomyces cerevisiae. J. Biol. Chem. 271: 23203-10.

Zhao H, Eide D. 1996b. The yeast ZRT1 gene encodes the zinc transporter protein of a high-affinity uptake system induced by zinc limitation. Proc. Natl. Acad. Sci. U.S.A. 93: 2454-8.

Zhao H, Eide DJ. 1997. Zap1p, a metalloregulatory protein involved in zinc-responsive transcriptional regulation in Saccharomyces cerevisiae. Mol. Cell Biol. 17: 5044-52.

Zhao H, Butler E, Rodgers J, Spizzo T, Duesterhoeft S, Eide D. 1998. Regulation of zinc homeostasis in yeast by binding of the ZAP1 transcriptional activator to zinc-responsive promoter elements. J. Biol. Chem. 273: 28713-20.

Zheng Z, Zou J. 2001. The initial step of the glycerolipid pathway: identification of glycerol 3phosphate/dihydroxyacetone phosphate dual substrate acyltransferases in Saccharomyces cerevisiae. J. Biol. Chem. 276: 41710-6.

Zhong Q, Greenberg ML. 2003. Regulation of phosphatidylglycerophosphate synthase by inositol in Saccharomyces cerevisiae is not at the level of PGS1 mRNA abundance. J. Biol. Chem. 278: 33978-84.

Zinser E, Paltauf F, Daum G. 1993. Sterol composition of yeast organelle membranes and subcellular distribution of enzymes involved in sterol metabolism. J. Bacteriol. 175: 2853-8.

Zinser E, Sperka-Gottlieb CD, Fasch EV, Kohlwein SD, Paltauf F, Daum G. 1991. Phospholipid synthesis and lipid composition of subcellular membranes in the unicellular eukaryote Saccharomyces cerevisiae. J. Bacteriol. 173: 2026-34.

Zou Z, Tong F, Faergeman NJ, Børsting C, Black PN, DiRusso CC. 2003. Vectorial acylation in Saccharomyces cerevisiae. Fat1p and fatty acyl-CoA synthetase are interacting components of a fatty acid import complex. J. Biol. Chem. 278: 16414-22.

Zweytick D, Athenstaedt K, Daum G. 2000a. Intracellular lipid particles of eukaryotic cells. Biochim. Biophys. Acta 1469: 101-120.

Zweytick D, Leitner E, Kohlwein SD, Yu C, Rothblatt J, Daum G. 2000b. Contribution of Are1p and Are2p to steryl ester synthesis in the yeast Saccharomyces cerevisiae. Eur. J. Biochem. 267: 1075-82. 


\section{List of figures, tables and abbreviations}

\section{List of figures}

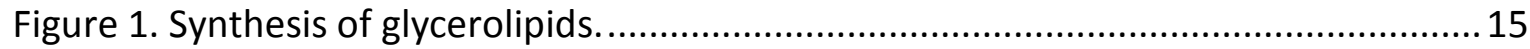

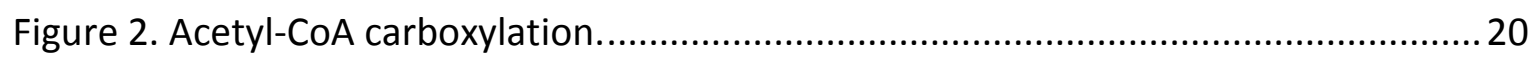

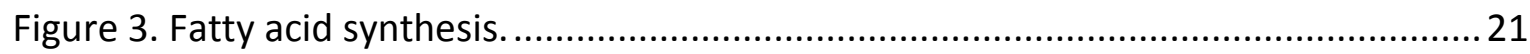

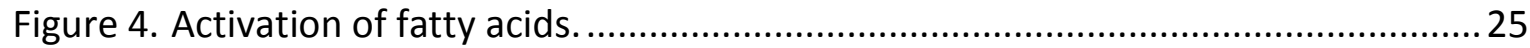

Figure 5. PCR synthesis of deletion cassettes and gene deletion by homologous

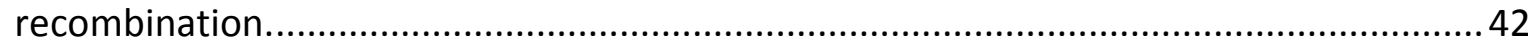

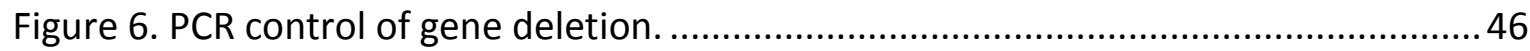

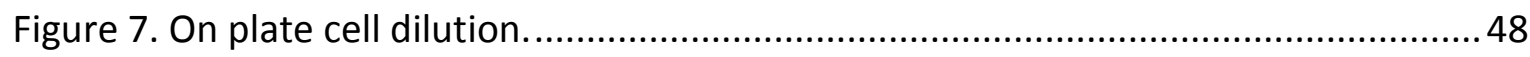

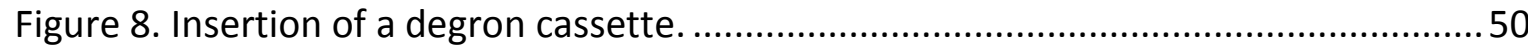

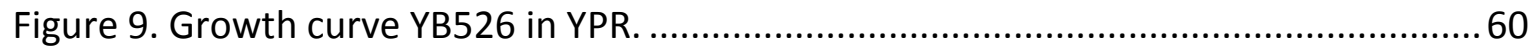

Figure 10. Number of cells per $\mathrm{ml}$ per $\mathrm{OD}_{600}$ unit at late stationary phase........................64

Figure 11. Free and bound FA in wild type (YB332) and acyl-CoA synthetase deficient

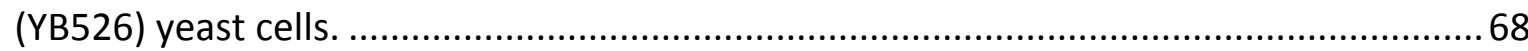

Figure 12. Free and bound FA in PLB deficient YB526 cells.............................................68

Figure 13. Deletions of the PDAT LRO1 or the TAG lipase TGL3 on YB526 cells. ...............69

Figure 14. Single and multiple deletions of the TAG lipases TGL2, TGL3, TGL4 and TGL5 on

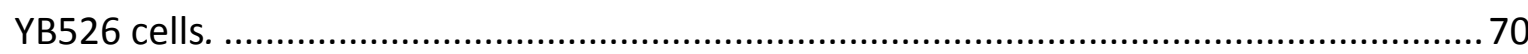

Figure 15. TAG metabolism in FFA production............................................................. 71

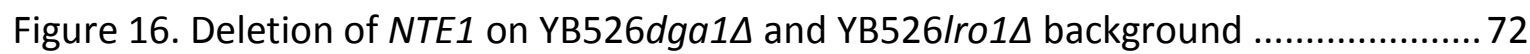

Figure 17. Free and bound FA in STE synthase and hydrolase deficient cells...................73

Figure 18. Simultaneous interference with TAG and STE metabolism............................. 74

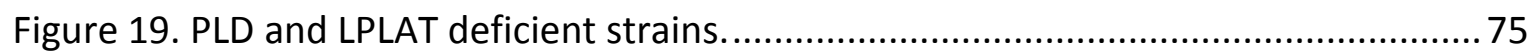

Figure 20. Free and esterified FA in autophagy deficient strains....................................77

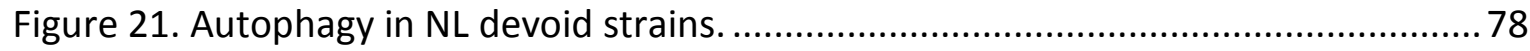

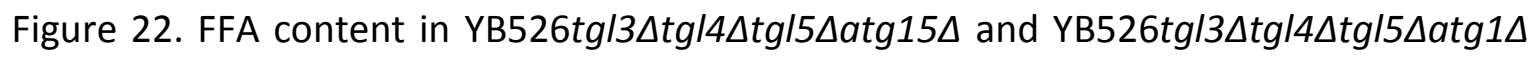

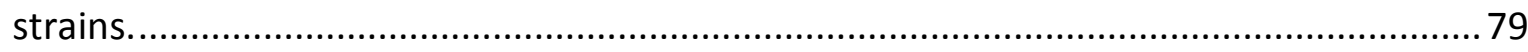

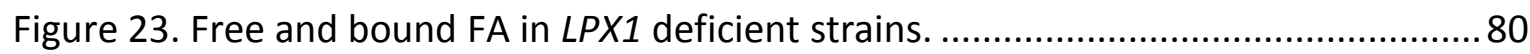




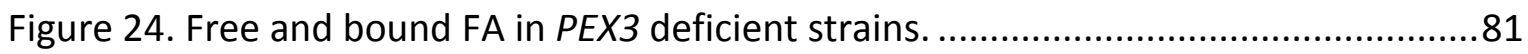

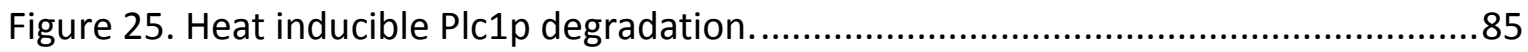

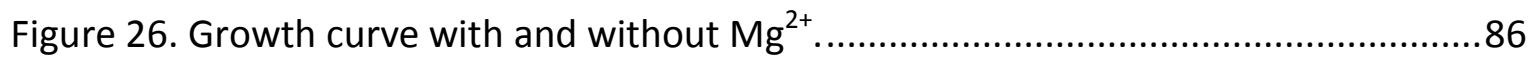

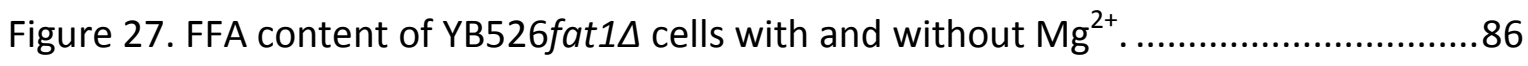

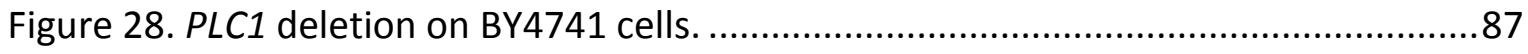

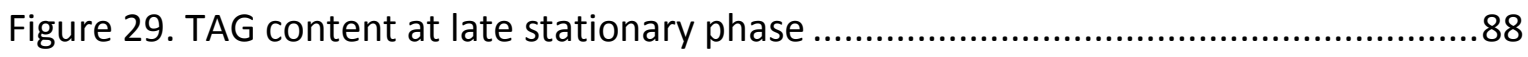

Figure 30. Quantification of various lipid classes......................................................... 90

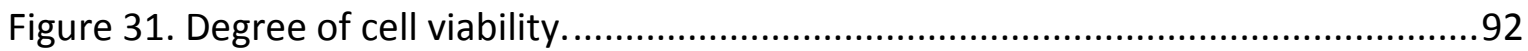

Figure 32. PLBs are not required for lysoPE deacylation............................................ 93

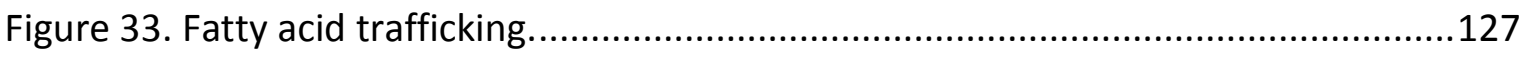

\section{List of Tables}

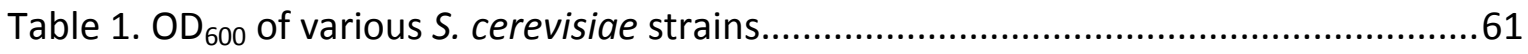

Table 2. Relative composition of the esterified and free fatty acid pools.........................82

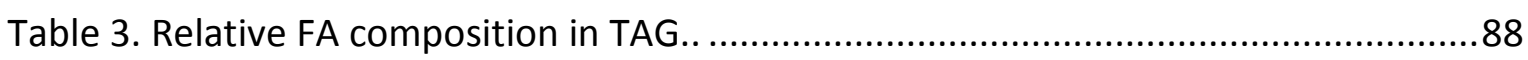

Table 4. Absolute composition of the esterified fatty acid pool....................................183

Table 5. Absolute composition of the free fatty acid pool. ...........................................185

\section{List of abbreviations}

$\begin{array}{ll}\text { 3MA } & \text { 3-methyladenine } \\ \text { 5-FOA } & \text { 5-fluoro-orotic acid } \\ \text { ACP } & \text { Acyl carrier protein } \\ \text { ACS } & \text { Acyl-CoA synthetase(s) } \\ \text { acyl-CoA } & \text { Acyl-Coenzyme A } \\ \text { CDP-DAG } & \text { Cytidine diphosphate diacylglycerol } \\ \text { CFU } & \text { Colony-forming unit } \\ \text { CL } & \text { Cardiolipin } \\ \text { CoA } & \text { Coenzyme A } \\ \text { CVt } & \text { Cytoplasm to vacuole targeting } \\ \text { DAG } & \text { Diacylglycerol }\end{array}$




\begin{tabular}{|c|c|}
\hline DCV & Degree of cell viability \\
\hline DGAT & DAG acyltransferase \\
\hline DGPP & Diacylglycerol pyrophosphate \\
\hline DHAP & Dihydroxyacetone phosphate \\
\hline DMPE & Dimethyl-PE \\
\hline EDAC & 1-Ethyl-3-(3-Dimethyl-aminopropyl)carbodiimid \\
\hline ELM & Exogenous lysolipid metabolism \\
\hline ER & Endoplasmic reticulum \\
\hline FA & Fatty acid(s) \\
\hline FAS & Fatty Acid Synthase \\
\hline FFA & Free fatty acid(s) \\
\hline G3P & Glycerol-3-phosphate \\
\hline GC & Gas chromatography \\
\hline GPC & Glycerophosphocholine \\
\hline GPD & Glycerophosphodiester(s) \\
\hline GPE & Glycerophosphoethanolamine \\
\hline KS & Ketoacyl synthase \\
\hline LC-MS & Liquid chromatography - mass spectrometry \\
\hline loxP & Locus of X-over P1 \\
\hline LP & Lipid particle(s) \\
\hline LPA & Lysophosphatidic acid \\
\hline LPAAT & Lysophosphatidic acid acyl transferase \\
\hline LPLAT & Lysophospholipid acyltransferase \\
\hline MAM & Mitochondrial associated membrane \\
\hline MM & Minimal media \\
\hline MMPE & Monomethyl-PE \\
\hline NAPE & N-acylphosphatidylethanolamine \\
\hline NL & Neutral lipid(s) \\
\hline$O D_{600}$ & Optical density at $600 \mathrm{~nm}$ \\
\hline ORF & Open reading frame \\
\hline
\end{tabular}




\begin{tabular}{ll} 
PA & Phosphatidic acid \\
PAS & Pre-autophagosomal structure \\
pbs & Primer binding sites \\
PC & Phosphatidylcholine \\
PDAT & Phospholipid:DAG acyltransferase \\
PE & Phosphatidylethanolamine \\
PG & Phosphatidylglycerol \\
PGP & Phosphatidylglycerol phosphate \\
PI & Phosphatidylinositol \\
PL & Phospholipid(s) \\
PLB & Phospholipase B \\
PMP & Peroxisomal membrane protein \\
PS & Phosphatidylserine \\
SN & Supernatant \\
STE & Steryl esters \\
TLC & Thin layer chromatography \\
YPD & Yeast peptone dextrose \\
YPR & Yeast peptone raffinose \\
\hline
\end{tabular}




\section{Appendices}

\section{Appendix 1. S. cerevisiae strains}

\section{Parental strains}

W303: wild type. MAT a, trp1, his3, ura3, leu2

(Thomas and Rothstein 1989)

MF17: W303 plb1::TRP1, plb2::HIS3, plb3::KANMX4

(Merkel et al. 1999)

YB332: wild type. MAT a NMTI, ura3, ade2, lys2, leu2, his3

(Johnson et al. 1994a)

YB524: YB332 faa4::LYS2

(Johnson et al. 1994a)

YB526: YB332 faa1::HIS3, faa2::LEU2, faa3::LEU2, faa4::LYS2

(Johnson et al. 1994a)

MS51: YB526 fat1::BLE

(Scharnewski et al. 2008)

BYP: $\quad$ BY4741 (Mat a; his3, leu2, met15, ura3) plc1::KANMX4

From EUROSCARF

\section{Strains generated within this study}

The deleted ORF is indicated in lower case, followed by the marker employed for deletion in upper case. As indicated before, markers were often removed to be reutilized, in these cases the deleted ORF is still indicated in lower case and the marker is removed from the description. In the main body of the thesis only the genes deleted, and not the markers employed, are indicated. Furthermore, with the purpose of increasing the readability of figures, the labels on figures indicate gene names only once, followed by the corresponding string of numbers. For example the strain YB526 tg/3::NAT loxp $\operatorname{tg} 14:: H Y G$

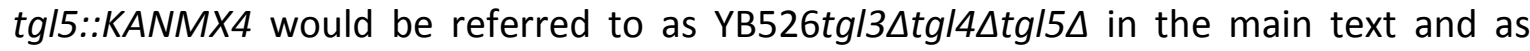
YB526tg/3,4,5 (or even as $\operatorname{tg} / 3,4,5 \Delta$ if there is no risk of confusion regarding the background) in figures.

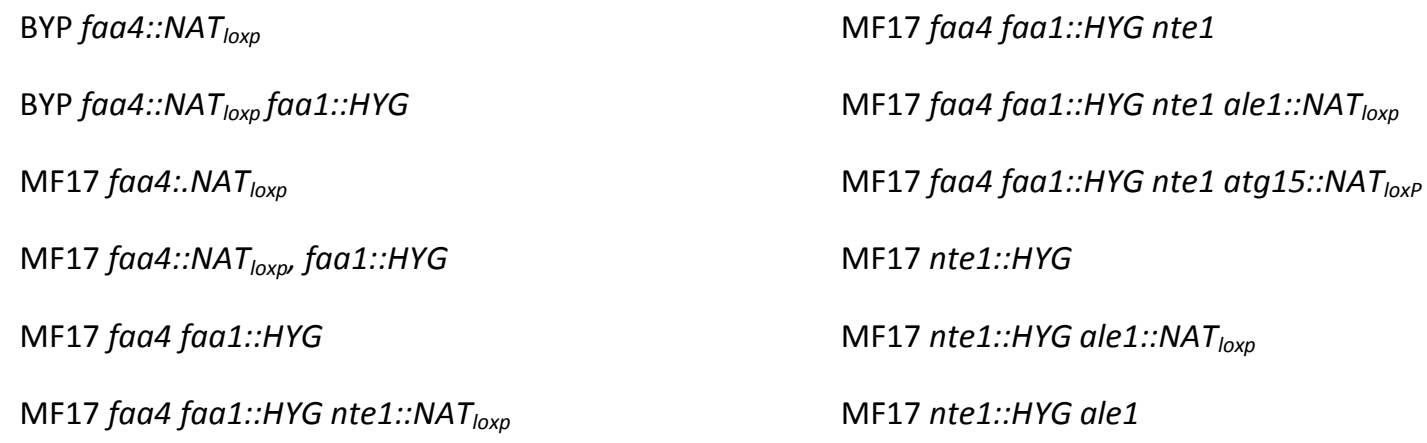


MF17 nte1::HYG ale1 psd1::NAT loxp

MF17 nte1::HYG ale1 psd1::NAT loxp $p s d 2:: U R A$

MF17 nte 1::HYG ale1 psd1::NAT loxp $p s d 2:: U R A$ atg15::LEU

MF17 nte1::HYG ale1 psd1

MF17 nte1::HYG ale1 psd1 psd2::NAT loxp

MF17 nte1::HYG ale1 psd1 psd2::NAT loxp $\operatorname{atg} 15:: L E U$

MF17 nte1::HYG ale1 psd1 psd2::NAT loxp atg15::URA

MF17 nte1::HYG atg15::LEU

MS51 ale 1::NAT loxp

MS51 dga1::NAT loxp

MS51 dga1::NAT loxp $n t e:: H Y G$

MS51 Iro1::NAT Ioxp

MS51 Iro1

MS51 Iro1 dga::HYG

MS51 Iro1 nte::HYG

MS51 nte1::NAT loxp

MS51 tg/3::NAT loxp

MS51 tg/3::NAT loxp $\operatorname{tg} / 4:: H Y G$

MS51 tgl3::NAT $T_{\text {loxp }} \operatorname{tg} 14:: H Y G$ tgl5::KANMX4 $4_{\text {loxp }}$

YB332 atg15:: $N A T_{\text {loxp }}$

YB332 tgl3:: NAT loxp

YB524 fat1::HYG

YB524 fat1::HYG UBR1::HIS3

YB524 fat1::HYG UBR1::HIS3 faa1::NAT loxp

YB524 fat1::HYG UBR1::HIS3 faa1::NAT loxp plc1::DEGRON(KANMX4)PLC1

YB524 fat1::HYG UBR1::HIS3 faa1::NAT loxp $_{\text {H }}$ plc1::DEGRON(KANMX4)PLC1 Iro1::LEU

YB524 UBR::HIS3

YB526 ale1::NAT loxp

YB526 are2::HYG
YB526 are2::HYG are1::NAT loxp

YB526 atg1::NAT $T_{\text {loxp }}$

YB526 atg1::NAT loxp $\operatorname{atg} 15:: H Y G$

YB526 atg1::NAT loxp $I p x 1:: H Y G$

YB526 atg1::NAT loxp pep4::HYG

YB526 atg1::NAT loxp pex3::HYG

YB526 atg15::NAT $T_{\text {loxp }}$

YB526 atg15::NAT $T_{\text {loxp }}$ Ipx1::KANMX4

YB526 atg15::NAT loxp Ipx1::KANMX4 tgl3::HYG

YB526 atg15::NAT loxp pex3::HYG

YB526 atg15::NAT $T_{\text {loxp }}$ tgl3::HYG

YB526 atg15::NAT $T_{\text {loxp }} \operatorname{tg} 14:: H Y G$

YB526 atg22::NAT loxp

YB526 atg22::NAT loxp $\operatorname{atg} 1:: H Y G$

YB526 atg22::NAT loxp $\operatorname{atg} 15:: H Y G$

YB526 dga1::NAT loxp

YB526 ire1::NAT loxp

YB526 Iro1::KANMX4 ${ }_{\text {loxp }}$

YB526 Iro1::kanmx4

YB526 Iro1::kanmx4 atg15::HYG

YB526 Iro1::kanmx4 atg15::HYG nte1::NAT IoxP YB526 Iro1::kanmx4 atg15::HYG tgl3::NAT loxP

YB526 Iro1::kanmx4 dga1::KANMX4

YB526 Iro1::kanmx4 dga1::KANMX4 are2::HYG

YB526 Iro1::kanmx4 dga1::KANMX4 are2::HYG are 1::NAT $T_{\text {loxp }}$

YB526 Iro1::kanmx4 dga1::KANMX4 are2::HYG are 1::NAT loxp $\operatorname{tg} 13:: U R A$

YB526 Iro1::kanmx4 dga1::KANMX4 are2::HYG are1::nat

YB526 Iro1::kanmx4 dga1::KANMX4 are2::HYG are 1::nat atg1::NAT loxp

YB526 Iro1::kanmx4 dga1::KANMX4 are2::HYG are1::nat atg1::NAT loxp pex3::BLE 
YB526 Iro1::kanmx4 dga1::KANMX4 are2::HYG are1::nat atg1::NAT loxp pex3::URA

YB526 Iro1::kanmx4 dga1::KANMX4 are2::HYG are1::nat atg15::NAT $T_{\text {loxp }}$

YB526 Iro1::kanmx4 dga1::KANMX4 are2::HYG are1::nat Ipx1::NAT $T_{\text {loxp }}$

YB526 Iro1::kanmx4 dga1::KANMX4 are2::HYG are1::nat nte $1:: N A T_{\text {loxp }}$

YB526 Iro1::kanmx4 dga1::KANMX4 are2::HYG are1::nat pex3::NAT $T_{\text {loxp }}$

YB526 Iro1::kanmx4 nte1::HYG

YB526 Iro1::kanmx4 nte1::HYG dga1::NAT loxp

YB526 Iro1::kanmx4 nte1::HYG tgl3::NAT loxP

YB526 Iro1::kanmx4 spo14::HYG

YB526 Iro1::kanmx4 tgl3::HYG

YB526 Ipx1::NAT Ioxp

YB526 nte1::NAT loxp

YB526 nte1

YB526 nte1 ale1::NAT loxp

YB526 nte1 ale1

YB526 nte1 atg15::NAT loxp

YB526 nte1 dga1::KANMX4 $4_{\text {loxp }}$

YB526 nte1 dga1::NAT loxp

YB526 nte1 spo14::HYG

YB526 nte1 tgl3::NAT

YB526 nte1 tgl3::NAT loxp atg15::HYG

YB526 nte1 tgl3::NAT loxp atg15::HYG Iro1::KANMX4

YB526 nte1 tgl3::NAT loxp $\operatorname{tg} 14:: H Y G$

YB526 nte1 tgl3::NAT loxp $\operatorname{tg} 14:: H Y G$

$\operatorname{tg} 15:: K A N M X 4_{\text {loxp }}$

YB526 рер4::NAT loxp

YB526 pep4::NAT loxp $\operatorname{atg} 15:: H Y G$

YB526 pep4::NAT loxp atg22::HYG
YB526 pex3::NAT

YB526 plb1::HYG

YB526 plb2::NAT IoxP

YB526 plb2::NAT $T_{\text {loxP }}$ plb1::HYG

YB526 plb2::NAT $T_{\text {loxP }}$ plb3::HYG

YB526 plb2::NAT $T_{\text {loxP }}$ plb3::HYG plb1::KANMX4 $4_{\text {loxP }}$

YB526 plb3::HYG

YB526 plc1::KANMX4 $4_{\text {loxp }}$

YB526 spo14::NAT loxp

YB526 taz1::NAT loxp

YB526 tgl1::NAT loxp

YB526 tgl2::NAT loxp

YB526 tgl3::NAT loxp

YB526 tgl3::NAT loxp $\operatorname{atg} 1:: H Y G$

YB526 tg/3::NAT loxp $\operatorname{atg} 15:: H Y G$

YB526 tgl3::NAT loxp $d g a 1:: H Y G$

YB526 tgl3::NAT $T_{\text {loxp }}$ Ipx1::KANMX4

YB526 tgl3::NAT loxp pex3::HYG

YB526 tgl3::NAT loxp $\operatorname{tg} 14:: H Y G$

YB526 tgl3::NAT $T_{\text {loxp }} \operatorname{tg} / 4:: H Y G \operatorname{tg} 15:: K A N M X 4$

YB526 tgl3 tgl4::HYG tg15::KANMX4

$\operatorname{atg} 1:: N A T_{\text {loxp }}$

YB526 tgl3 tgl4::HYG tg15::KANMX4

$\operatorname{atg} 15:: N A T_{\text {loxp }}$

YB526 tgl3 tgl4::HYG tgl5::KANMX4

YB526 tgl4::NAT loxP

YB526 tgl5::NAT loxP

YB526 yeh1::NAT loxp

YB526 yeh2:: $N A T_{\text {loxp }}$

YB526 yeh2::NAT $T_{\text {loxp }} \operatorname{tg} 11:: H Y G$

YB526 yeh2::NAT loxp $\operatorname{tg} 11:: H Y G$ yeh1::KANMX4

YB526 yeh2::NAT $T_{\text {loxp }}$ tgl1::HYG yeh1::KANMX4 $\operatorname{tg} / 3:: U R A$ 


\section{Appendix 2. Primer sequences}

Primers were ordered from Invitrogen. Desalted purity standard was used for synthesis of deletion cassettes and verification of ORF deletion / marker insertion. PAGE purity standard was used for synthesis of degron cassettes.

\section{Primers for synthesis of deletion cassettes}

AlE1 UP: $\quad$ ACAAACCGTGGTGATTTAATTCTGCTGCTGATCGCTTCCAACATGCGTACGCTGCAGGTCGAC ALE1 DO: $\quad$ ACAAGACTGTGACTTCCACACGCATCTGTCGTTTTTGGCCATCTAATCGATGAATTCGAGCTCG ARE1UP: TCCGCAGACTCAATTCCGCAGAAGCCAACAAACGGCATTCGGTCACGTACGCTGCAGGTCGAC ARE1DO: TCTTTGACCACGGTGGAGCGGACGCCCATTCCCTATTGTATATCTATCGATGAATTCGAGCTCG ARE2UP: CCAAAGAGAAGGCACGGTATAGGCAAGGGTCCTCTAACTTTATATCGTACGCTGCAGGTCGAC ARE2DO: TGCAGCATTTGGAAGAAGAACAAGTAAAACCTCAATTTCTTGAAGATCGATGAATTCGAGCTCG ATG1UP: TTTTCAAATCTCTTTTACAACACCAGACGAGAAATTAAGAAAATGCGTACGCTGCAGGTCGAC ATG1DO: CAGGTCATTTGTACTTAATAAGAAAACCATATTATGCATCACTTAATCGATGAATTCGAGCTCG ATG15UP: TAGGCATTACAATTAAAGGAAACAAGGGAAATATTCTATTGAATGCGTACGCTGCAGGTCGAC ATG15DO: $\quad$ TTCCAGTTGTAGCAATCTACGCAGGGCTCTGGTTCAACGCACTTGATCGATGAATTCGAGCTCG ATG22UP: $\quad$ TATAAGCAATAGTTTGCTCATAACATATTCTCTACATTAGATATGCGTACGCTGCAGGTCGAC ATG22DO: GAGTTCTTCAGCCTCTCTTCTACCTCTCTTGACATCCAAACAGTTATCGATGAATTCGAGCTCG DGA1UP: CTGTAGCATGGCACACTTCTTCATTTGTACTCTTCTCCATATTTACGTACGCTGCAGGTCGAC DGA1DO: TCCAACAACAACATTGATAGGCGCTCTAAATGGCAACAAACCGAAATCGATGAATTCGAGCTCG FAA1UP: TAAAAACTAGAACAAACACAAAAGACAAAAAAAGACAACAATATGCGTACGCTGCAGGTCGAC FAA1DO: TTTAGTATGATGAGGCTTTCCTATCATGGAAATGTTGATCCATTAATCGATGAATTCGAGCTCG FAA4UP: GTTCTTCACTATTTCTTGAAAAACTAAGAAGTACGCATCAAAATGCGTACGCTGCAGGTCGAC FAA4DO: TTTATGAAGGGCAGGGGGGAAAGTAAAAAACTATGTCTTCCTTTAATCGATGAATTCGAGCTCG FAT1UP: $\quad$ ATTCTATATCGTTGAACTTTTAATAGGCTGCGAATACCGACTATGCGTACGCTGCAGGTCGAC FAT1DO: CATCCAAACCCTTTGGTAATTTTTGCTCTCTATAAACCTTCTTCAATCGATGAATTCGAGCTCG IRE1UP: $\quad$ AAACAGCATATCTGAGGAATTAATATTTTAGCACTTTGAAAAATGCGTACGCTGCAGGTCGAC IRE1DO: ATGCAATAATCAACCAAGAAGAAGCAGAGGGGCATGAACATGTTAATCGATGAATTCGAGCTCG LPXIUP: $\quad$ TGCTTATCTCTGCGTATCCGCCGATCAACATCCGCGGCTAAAATGCGTACGCTGCAGGTCGAC LPX1DO: $\quad$ ACCAGGTCCGGAGCCTCAACGTTGACCAAGTGGGAGCCGCCGGGAATCGATGAATTCGAGCTCG 
LRO1UP: CGTGGCAAAGATTTCGACAGGAAAAGAGACGGGAACGGTAGAAAACGTACGCTGCAGGTCGAC LRO1DO: GGATGTCTACGTGTTCGGCGCTTTTTGCTCCACCACGTATATCAAATCGATGAATTCGAGCTCG NTE1 UP: $\quad$ TTCAACTTCTCGTGGGTTGTGTCCTACTTTGTTATGGGTGCCTCTCGTACGCTGCAGGTC NTE1 DO: ACCAAATCATAATCCTTCGCGTACAAACCGCCAACAAAGGAACCAATCGATGAATTCGAG PEP4UP: GTATTTAATCCAAATAAAATTCAAACAAAAACCAAAACTAACATGCGTACGCTGCAGGTCGAC PEP4DO: TAAATAGAATAGTATTTACGCAAGAAGGCATCACCAACGATGGCCATCGATGAATTCGAGCTCG PEX3UP: TAAAAGCAGAAGCACGAAACAAGGAGGCAAACCACTAAAAGGATGCGTACGCTGCAGGTCGAC PEX3DO: $\quad$ TATATATATATTCTGGTGTGAGTGTCAGTACTTATTCAGAGATTAATCGATGAATTCGAGCTCG PLB1UP: GAACGTTAACGCTTGGTCACCAAATAACAGTTACGTCCCTGCGAACGTACGCTGCAGGTCGAC PLB1DO: GAAGAATAATCATCATTTCCTACACCTGAGACTGACCTGCTGTCAATCGATGAATTCGAGCTCG PLB2UP: TACAGGCTAGCTCGCTAATTTCTGGACTTTCGCTCGCTGCAGATTCGTACGCTGCAGGTCGAC PLB2DO: GAACCTAAGAGAGCCGTTATTGGAATACCGTCAGTAGCCTCAGAGATCGATGAATTCGAGCTCG PLB3UP: TCAGTTACATATTCGCAATTTCTCAGTTTCTACTGGCCGCTAATGCGTACGCTGCAGGTCGAC PLB3DO: GCATAATCATAGCAGAGAACTTTACACTTATGCCAGATAAATGTGATCGATGAATTCGAGCTCG PLC1 UP: TAACAAGAAATTAAGAAAAGACTGTGATCTCAGCACGCTATGTCGCGTACGCTGCAGGTC PLC1 DO: $\quad$ AATCCTTGTACCCTTATCGATTGAGATGGGCATTGTCGCCTCGTCATCGATGAATTCGAG PSD1 UP: $\quad$ TTGGTCGTTATTTTTTGAAGAAGAAGGAAAAGCAAAGCCAGCATGCGTACGCTGCAGGTCGAC PSD1 DO: $\quad$ TATATACAGCAAAATAAATGCTAACTTTACATATGATTGCTTTCAATCGATGAATTCGAGCTCG PSD2 UP: TGGTAAAGAATCCTCGATTTTCAGGAGCATCCAACGACGAAGATGCGTACGCTGCAGGTCGAC PSD2 DO: $\quad$ CCATTTTGGTAACCACTAACTACAGCCAATTTTTCGGCGGCTTCAATCGATGAATTCGAGCTCG SPO14 UP: $\quad$ CCACGCTTTTAAGAAAATCTCTGCTATTGGTAGACTGAAATCCAGCGTACGCTGCAGGTC SPO14 DO: CATAATTAAACGAAGACTTGAGGCTTCTGCAGTATCTACTGGAGAATCGATGAATTCGAG TAZ1 UP: $\quad$ TTTCATTTTCAAAAAAAAAAAAAGTAAAGTTTTCCCTATCAAATGCGTACGCTGCAGGTCGAC TAZ1 DO: $\quad$ CTCATACATGCTAGTATTTACACGAATTTAATTGCTTAAATTTCAATCGATGAATTCGAGCTCG TGL1UP: $\quad$ TTATTATTCTAGCACTATTTTAAAAAACTGTCTTTTGGCAAAATGCGTACGCTGCAGGTCGAC TGL1DO: CTAGACAAAAAATAGTTTAATAGGGTTTCTCTCGCATTCTTTTCAATCGATGAATTCGAGCTCG TGL2UP: AATCCCTTCTGTATTCCATCTGACAAACCTAATTTCCAATTCAATCGTACGCTGCAGGTCGAC TGL2DO: TTGGCGTAACCAAATTGAAATATTTCATATACGCAGTCGTGAGTTATCGATGAATTCGAGCTCG TGL3UP: GTTAGATGAGTGTGATACGTATCAAATGTGGTGTCAGCAAGCGTCCGTACGCTGCAGGTCGAC TGL3DO: TTCAACTGCGGTATGCCCAATTCTGGGATTATGTACTCTGCTGAAATCGATGAATTCGAGCTCG TGL4UP: GCAAGGGTCATCTTTAGCCAGTAAATGCAAATCATTTCTTTACAACGTACGCTGCAGGTCGAC TGL4DO: ACGCCTGAAATTGCCCGCTAAATCACTATGAATCATTGCGTCTGAATCGATGAATTCGAGCTCG 
TGL5UP: GAGTCCTCGTTGTATAACTATAAGCTGCTGCAAGACTTGACCATCCGTACGCTGCAGGTCGAC TGL5DO: GCAAGTCAGCATCCATTATGGAAAGAGATTTGATTTGCTCACTGAATCGATGAATTCGAGCTCG YEH1UP: $\quad$ AATAGTTTTATATATAGGTATATTTACTGCACAATTCACACGATGCGTACGCTGCAGGTCGAC YEH1DO: CCAGATCTTGTAGTTGACATTCGACTCCACATTGACGAAATGATTATCGATGAATTCGAGCTCG YEH2UP: $\quad$ AACGCCCTTAGATTTACAGCGGGATCAGGAAAATAATATTGAATACGTACGCTGCAGGTCGAC YEH2DO: TATTATATTTTACAAAGAAACCACAAAGAAAAAACTTTTACCTCAATCGATGAATTCGAGCTCG

\section{Primers for synthesis of a PLC1-specific degron cassette}

DPLC1up:

AATAGTATAAGCATCACGACATAAGCAGCCTCAGGTCATTAAAACAATAAATTAAGGCGCGCCAGATCTG DPLC1do:

AACTCCTTTGTAAGATTAAACCTTTGGTCATCTATAGCACTTTCAGTCATGGCACCCGCTCCAGCGCCTG

\section{ORF specific primers for control of gene deletion / construct insertion}

ALE1A: TGtCCTGCTGTTGAgCtgtTTtGCT

AlE1C: TtCTgCAAgCtCTCCAAACTTGgGt

ARE1A: CGTGGTTGGACTCGTTTAGCGAACA

ARE1C: GCAAGAGTGGTTCCACCTGCTCTTC

ARE2A: ACACAACCATGGACAAGAAGAAGGA

ARE2C: GATGTATCCTGTAGCAATGAGAGCA

ATG1A: CCGCTCGGCTCTGATTTCTTAAACC

ATG15A:GTTGTTCGCTGAAGACAGCCGTTTC

ATG15C:ACTGGGAGGCGCATTGGCCAGTTTA

ATG22A:TGTATTGTGCGGCATTGATAGCGCC

ATG22C: GGTTGATTGACGATGTGACAATACC

DGA1A: TAACCAAGCACGACAGTGGTCTATC

DGA1C: GCGTTTGGAGCGTTTGCAACAGAAG

FAA1A: ATGATACAGGCACGAATACATCATA

FAA1C: CTAAAAAGTTGgGAATTATGGAACA

FAA4A: TCGTTCATCTCGTTCTTTCTACTCT
ALE1B: GTGCATGAACCATCATAGTACGACC

ALE1D: GCTAAAGAAGTTGGTGATGAGGCAG

ARE1B: CCTTGGTCTTGGTCTTTGTCAACTG

ARE1D: CGTTCATTTTGTCGTCACAACACCT

ARE2B: TCCTTGAAGCTACCATTATGCTGGT

ARE2D: AGCTCCACAGAACAGTTGCAGGATG

ATG1B: GTCCCCAAGAGCACAGTACTCCATG

ATG15B:CAAAATCGAATGGGTTAGTGGTGGC ATG15D:AGGTAGTAGTAGCAGTGGTGCGAGT

ATG22B:GACAATGCGCCCACAATACCAAACC ATG22D:AATGTTTGCTTCTGGCGGTGATGAC DGA1B: TCCTGGTTGCGATAGTCAATAGTAG DGA1D: CAGTTACGCTTTGCCTGGTAAGCTA FAA1B: TGCACTGGTTTGATCAAGGATGGTA FAA1D: GGTAATGCTTCTTATGTGAGGTGTC FAA4B: TCTTTTGTCACTTGGATCGATGGGT 
FAA4C: TTCTTTGAAGAAGAATGGACTCCAG FAT1A: TTGAGCGgATATTTCAGAATGTCAA FAT1C: CGGTTAAATCTACCGTCTTATGCTA IRE1A: ATCAAGACGGAGCGTAAGCCTCTTC LPX1A: TTCTCCGCAATCTCGGAGTTAGTGC LRO1A: CCAACGAATTCGGCGACAATCGAGT LRO1C: ACACCACAAGCACTGGTCGAATCCA NTE1A: CGAAGGCAACCTTTACGTATTTGCC NTE1C: CCTCCAACGAAAGCGTCTTACTCTA PEP4A: TGAGAAGCCTACCACGTAAGGGAAG PEX3A: GTCGTAGTCTATGCGTTTGTAGTGA PLB1A: CCACATTTAACCAACGGAAACGTCT PlB1C: TGgtTGATGgtgGtgAAGATAACCA PLB2A: CCGCATATTCTCCACCTTGGCATTG PLB2:C TTCTAAGCAAGGTAAAGGAATGGCT PLB3A: GCACTTCTTTTGCAAGATCCAGACT PLB3C: GCGCGTTCAAGCTATCATATTCCGA PLC1A: AAGCATCACGACATAAGCAGCCTCA PSD1A: CGACCAATTGTGCTTCTGTATACGC PSD1C: GCTACCAGGCAGTATATGAGAATGC PSD2A: CCTAACGCATGTGCTACTTCAAGGA PSD2C: CCGTTCGTAGTGAGTTAGACGTCTT SPO14A:TGTGTCACCGAACCACTCATTAGAC TAZ1A: TCTTCTACCTTCACTCAGACCGAGA TGL1 A: CCCAAAATGCCGATTAACACAAACC TGL2A: TACGCCACGCGAGCTACTATTTGTC TGL3A: TTCGGAGATACTTATCCTAGGTCTG TGL3C: CAGCCATTGAATGCTCACTTGGTTC TGL4A: CCTCCTTGTTACGCAACAACTAATC TGL4C: CCACATCAAGGATGTTGAGGCAATC TGL5A: AACAGCACAAGGAAGACGGTTCTGT TGL5C: CCACGAAGTATTGAAGAACCCATCT
FAA4D: GATGAGAAATCAAGAATGAAGCAAG FAT1B: ACCTATGTCTTCTCACATCAATGAT FAT1D: AACCATTCAGTTACCGATAGTTTCA IRE1B: TTCTTGGAGCAGGAATGgGCGAGTT LPX1B: ATGAAACAGATTGGGCTGCAGGACG LRO1B: GTAAAGTTCGGTGGGTCCAGACCTG LRO1D: TCCGCAGCCTACTTAGAAAACAGTG NTE1B: ATTGTTGACCGACACCGTTGTGTCT NTE1D: CTGATGCGACGTAACCCAATCTAAC PEP4B: AATGTTAAGCCCGGCTCGCTGGTAG PEX3B: ATTCTGTAGTTTGTTGCAGTTTTCC PLB1B: GTCCCATCTACTCCCAGTCTTAAAG PLB1D: AGgGAAGACACCCGGTATGTAAATG PLB2B: GAGCATGCAATACCAATTTTGGGTAC PLB2D: GCTGTTATGGCACCTAACAAACTCA PLB3B: GGCTAATGTACCAACTAGCCAGTTG PLB3D: GGTCTTGCCCGAAAGGGATGATACT PLC1B: GTTTGACGATCCACTTGCTCATGCT PSD1B: CATAATCGAGACATCGCATTCAGCG PSD1D: ATACCACCTCTTCGCAACTGGTTGA PSD2B: CCGGTGTAGCTGAAGATGATACTGA PSDD: TCAACGATGACTTGTTTGTACACGC SPO14B: AATGTATCGGTTGGGAGAAATCGTC TAZ1B: TGTTGAAAGgACTTGGCCAAGTGAG TGL1B: TTCGATGGTATTGTCATCGGAGGAC TGL2B : CGAATGTCGCTTATCCTTCGACTCG TGL3B: AGCCCAAACAATGAACCACCTTGCA TGL3D: AAAGCTAGTGCGGGCCATGTACTTC TGL4B: AGAGTACCAAGGACACCGATGTGGA TGL4D: GGCATTTCCTGCTTCGTAAGACTGA TGL5B: GGGAGATTTGGAATTGTCGTCATTG TGL5D: AGCCGgTATGgGAAAATCTTCTGAG 
YEH2A: GATTCACCATTGGCGTTGTTATCCC

YEH1A: CCTATTCATCAAGCTTCATACATCC
YEH2B: CTCTACGCCATCGTTTAACCTGGAC

YEH1B: GATCCGCAACTAATGTCAAGTCTTC

Marker specific primers for control of gene deletion / construct insertion

NATB: TACCGGTAAGCCGTGTCGTCAAG

HYGB: GATCAGAAACTTCTCGACAGAC

KANB: TGCAGACCTGCGAGCAGGGAAAC

BLEB: TCCGAAGCCCAACCTTTCATAGAAG

LEUB: TCTAGCTTCCCTACCTGACACTAAC

UBRA: TCCATTCTCAATTAGCTCTACCA
NATC: GCTGGAGGTCACCAACGTCAAC

HYGC: CGATGGCTGTGTAGAAGTACTC

KANC: GTGATTTTGATGACGAGCGTAATG

URAB: CAGCAGTACAGAACCGTCGACGAAG

DEGC: CTGGTGCAGGCGCTGGAGCG

UBRB: $\quad$ ATAGATATCTGTAAATGAACTCT 


\section{Appendix 3. Fatty acid profiles}

Table 4. Absolute composition of the esterified fatty acid pool. S. cerevisiae mutants grown in YPR media for $136 \mathrm{~h}$ at $30^{\circ} \mathrm{C}$. The minor species $14: 0$ and 18:1(11Z) are not presented in the table but are included in the total. All genes indicated in the table correspond to deletions on the background of the strain YB526.

Esterified fatty acids

Standard deviation

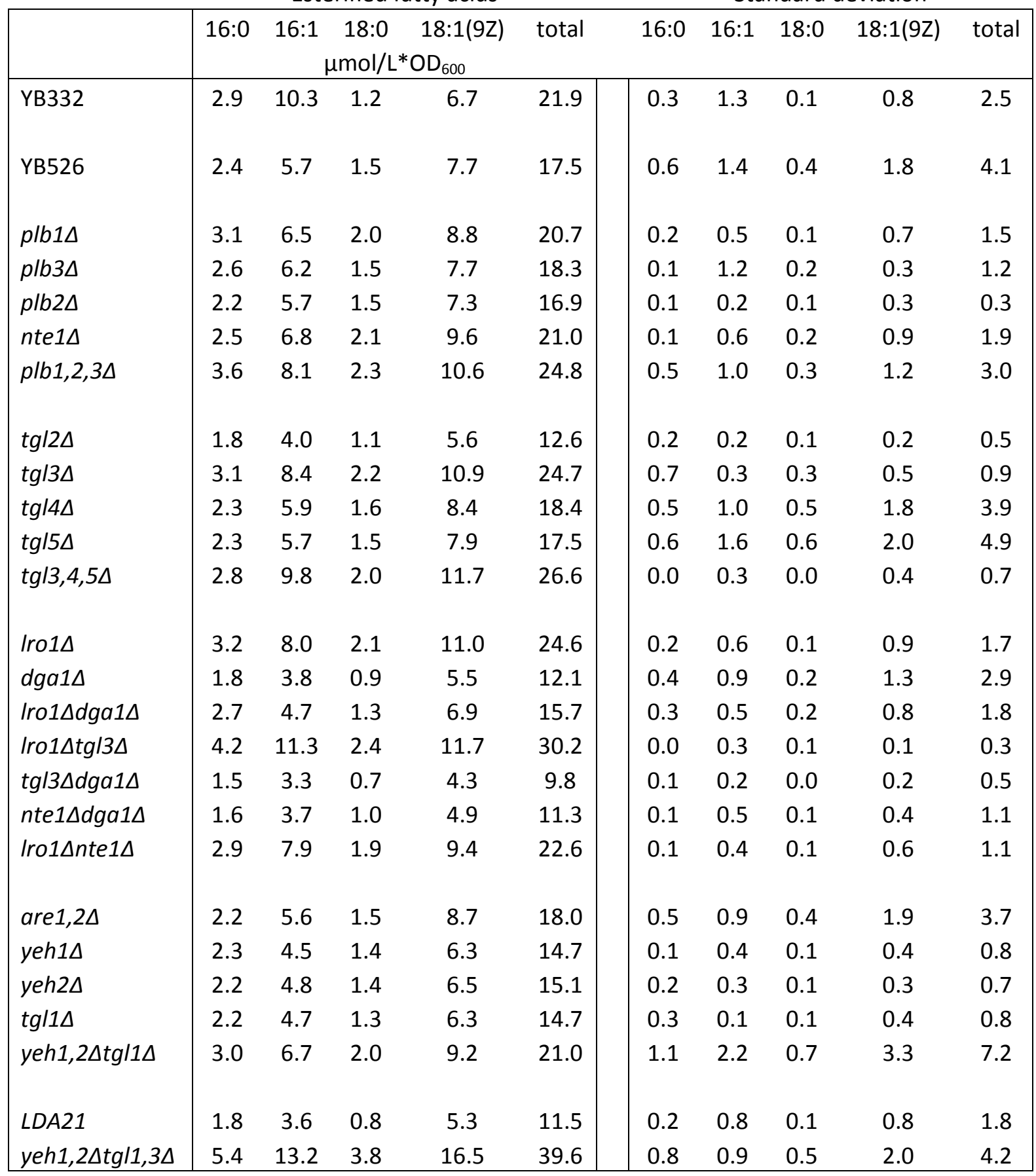


Table 4. Continued from previous page.

Esterified fatty acids

Standard deviation

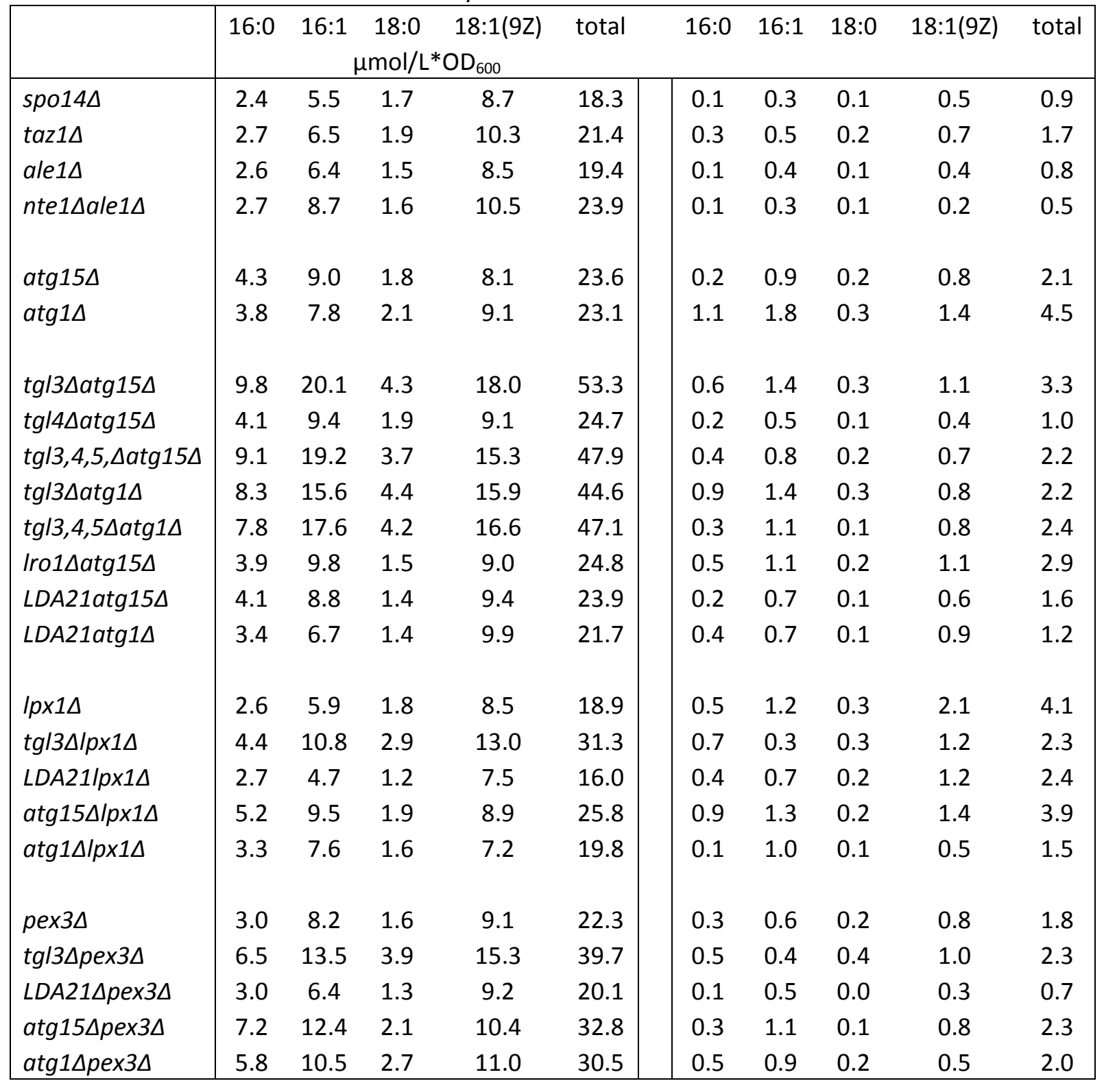


Table 5. Absolute composition of the free fatty acid pool. S. cerevisiae mutants grown in YPR media for $136 \mathrm{~h}$ at $30^{\circ} \mathrm{C}$. The minor species $14: 0$ and $18: 1(11 \mathrm{Z})$ are not presented in the table but are included in the total. All genes indicated in the table correspond to deletions on the background of the strain YB526.

Free fatty acids

Standard deviation

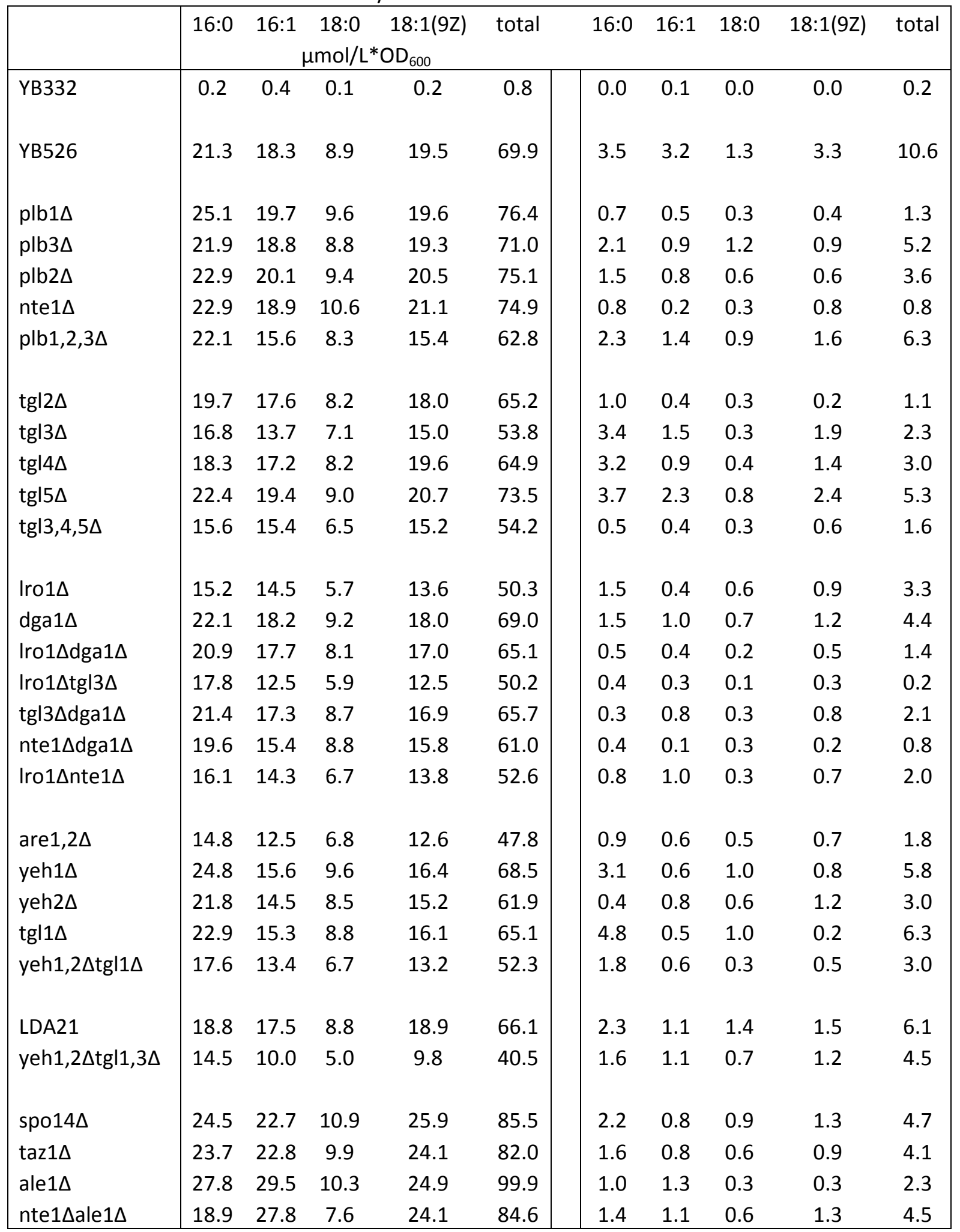


Table 5. Continued from previous page.

Free fatty acids

Standard deviation

\begin{tabular}{|c|c|c|c|c|c|c|c|c|c|c|}
\hline & $16: 0$ & $16: 1$ & $\begin{array}{c}18: 0 \\
\mathrm{~mol} / \mathrm{L}\end{array}$ & $\begin{array}{l}18: 1(9 Z) \\
D_{600}\end{array}$ & total & $16: 0$ & $16: 1$ & $18: 0$ & 18:1(9Z) & total \\
\hline $\operatorname{atg} 15 \Delta$ & 15.8 & 9.0 & 5.7 & 9.9 & 42.1 & 2.5 & 2.1 & 1.1 & 1.5 & 6.7 \\
\hline $\operatorname{atg} 1 \Delta$ & 17.2 & 10.4 & 5.9 & 11.6 & 46.6 & 3.5 & 0.9 & 0.7 & 0.5 & 4.6 \\
\hline $\operatorname{tg} \mid 3 \Delta \operatorname{atg} 15 \Delta$ & 14.7 & 4.2 & 3.6 & 5.3 & 29.3 & 1.9 & 0.3 & 0.5 & 0.3 & 2.9 \\
\hline $\operatorname{tg} \mid 4 \Delta \operatorname{atg} 15 \Delta$ & 15.4 & 8.1 & 5.9 & 8.9 & 39.5 & 0.5 & 0.4 & 0.4 & 0.3 & 1.5 \\
\hline $\operatorname{tg} \mid 3,4,5, \Delta \operatorname{atg} 15 \Delta$ & 11.5 & 3.8 & 2.5 & 4.3 & 23.3 & 0.6 & 0.1 & 0.2 & 0.1 & 0.6 \\
\hline $\operatorname{tg} \mid 3 \Delta \operatorname{atg} 1 \Delta$ & 14.2 & 4.9 & 4.1 & 6.5 & 30.6 & 5.8 & 1.5 & 1.5 & 1.2 & 10.4 \\
\hline $\operatorname{tg} 13,4,5 \Delta \operatorname{atg} 1 \Delta$ & 8.7 & 4.4 & 2.5 & 6.1 & 22.3 & 0.7 & 0.1 & 0.3 & 0.2 & 1.2 \\
\hline Iro1 $\Delta$ atg15 & 12.4 & 7.6 & 4.1 & 9.2 & 34.6 & 1.6 & 0.3 & 0.4 & 0.4 & 2.5 \\
\hline LDA21atg15 $\Delta$ & 13.9 & 5.9 & 4.6 & 5.7 & 31.4 & 1.6 & 0.3 & 0.4 & 0.3 & 2.0 \\
\hline LDA21atg1 $\Delta$ & 16.6 & 11.1 & 6.3 & 13.1 & 48.2 & 4.5 & 1.4 & 1.1 & 1.3 & 4.3 \\
\hline Ipx1s & 15.4 & 12.8 & 6.3 & 12.9 & 48.7 & 2.3 & 1.9 & 0.7 & 2.1 & 7.0 \\
\hline $\operatorname{tg}|3 \Delta| p x 1 \Delta$ & 17.1 & 11.5 & 5.9 & 11.6 & 47.3 & 2.0 & 0.3 & 0.4 & 0.1 & 2.6 \\
\hline LDA21/px1 $\Delta$ & 20.5 & 14.6 & 8.2 & 16.0 & 60.7 & 1.0 & 1.0 & 0.1 & 0.7 & 1.1 \\
\hline $\operatorname{atg} 15 \Delta \operatorname{lpx} 1 \Delta$ & 14.7 & 7.5 & 4.7 & 7.6 & 36.1 & 1.5 & 0.2 & 0.2 & 0.4 & 2.4 \\
\hline $\operatorname{atg} 1 \Delta \operatorname{lp} \times 1 \Delta$ & 17.2 & 8.1 & 5.7 & 9.9 & 42.0 & 4.3 & 1.2 & 1.3 & 1.0 & 8.0 \\
\hline $\operatorname{pex} 3 \Delta$ & 14.6 & 13.9 & 5.7 & 15.2 & 50.4 & 0.7 & 0.6 & 0.3 & 0.6 & 2.2 \\
\hline $\operatorname{tg} \mid 3 \Delta \operatorname{pex} 3 \Delta$ & 19.1 & 9.5 & 6.3 & 10.6 & 46.7 & 1.3 & 0.4 & 0.5 & 0.7 & 3.0 \\
\hline LDA21 $\Delta$ pex3 $\Delta$ & 15.4 & 14.1 & 6.7 & 15.1 & 52.6 & 2.1 & 0.9 & 0.5 & 1.3 & 4.5 \\
\hline $\operatorname{atg} 15 \Delta$ pex3 $\Delta$ & 15.4 & 6.2 & 4.7 & 7.3 & 35.0 & 0.8 & 0.2 & 0.1 & 0.2 & 1.3 \\
\hline $\operatorname{atg} 1 \Delta \operatorname{pex} 3 \Delta$ & 20.0 & 9.3 & 7.0 & 11.9 & 49.6 & 1.3 & 0.4 & 0.5 & 0.4 & 2.6 \\
\hline
\end{tabular}




\section{Acknowledgments}

I want to express my sincere gratitude to all the people that have taken part in making this work possible, and even more, that have made the experience of going through it an enjoyable and memorable one.

Professor Ivo Feußner, for receiving me in his department and for his permanent support and interest.

Dr. Martin Fulda, for his guidance and for always being disposed to share his time and advice.

Professor Michael Thumm and Professor Klaus-Armin Nave, for accepting to take part in my thesis committee and for their helpful suggestions and advice.

Michael Scharnewski, for his cooperation at work and his friendship beyond.

Dr. Annett Struß, Dr. Danuta Kaczmarzyk, Dr. Martin Wagner, Dirk Jessen, Eva Küch, Alexandra Andreou, Amina Ibrahim, Mareike Possienke and Florian Brodhun for their friendship that made life at work a pleasure.

All members of the Plant Biochemistry Department, for the pleasant and cooperative environment.

Ramya Nair, for her patience during my writing and for making my life a happy one. Larissa Yurlova, Chieh Hsu, Katharina Hoff and so many friends that have made Göttingen a true home.

Dr. Steffen Burkhardt and all members of the IMPRS MolBio/Neuro and GGNB coordination team for their outstanding work.

The Lichtenberg and GGNB stipendiums for financial support.

I very specially want to thank my family for their support and so much more. I would like to dedicate this work to my father. How I wish he could have read it. 


\title{
Curriculum vitae
}

\author{
Name: $\quad$ Gabriel Mora \\ Born in: Bogotá Colombia
}

13 June 1977

Nationality: Colombian / German

Contact: g.e.mora@gmail.com

1984-1989: Primary school:

Anglo-Colombian School. Bogotá, Colombia

1989 - 1995: Middle and High school, International Baccalaureate Diploma

Anglo-Colombian School. Bogotá, Colombia

1995 - 1996: Marine Corps, Colombian Navy

1996 - 2003: Professional degree (B.Sc.) in chemistry

National University. Bogotá, Colombia. Graduation work: Contribution to the knowledge on vegetal metabolic defense mechanisms. Director, Dr. Marta Lucia Guardiola; Codirector, Prof. Dr. Eduardo Posada.

2001 - 2004: Research assistant. Vegetal Biotechnology Group, International Physics Centre. Bogotá, Colombia. Principal investigator: Dr. Marta Lucia Guardiola.

2003 - 2004: Professional studies (B.A.) in literature

Universidad Javeriana. Bogotá, Colombia

2004 - 2006: M.Sc. degree in molecular biology

IMPRS MolBio, Georg-August-University. Göttingen, Germany. M.Sc. thesis: The Phospholipase B Chapter in the Metabolic Odyssey of a Free Fatty Acid in a Yeast Cell. Director, Prof. Dr. Ivo Feußner; Supervisor, Dr. Martin Fulda.

2006 - 2010: Ph.D. degree in molecular biology

IMPRS MolBio, Georg-August University. Göttingen, Germany. Ph.D. thesis: Pathways for phospholipid deacylation in Saccharomyces cerevisiae and their impact on fatty acid trafficking and equilibrium. Director, Prof. Dr. Ivo Feußner; Supervisor, Dr. Martin Fulda. 


\section{Publications}

Scharnewski M, Pongdontri P, Mora G, Hoppert M, Fulda M. 2008. Mutants of Saccharomyces cerevisiae deficient in acyl-CoA synthetases secrete fatty acids due to interrupted fatty acid recycling. FEBS J. 275: 2765-78.

Mora G, Scharnewski M, Fulda M. Neutral lipid metabolism influences the establishment of the phospholipid species composition in Saccharomyces cerevisiae. Under review.

Mora G, Thumm M, Fulda M. Autophagy in lipid turnover and equilibrium. In preparation. 